\title{
SERRE WEIGHTS AND BREUIL'S LATTICE CONJECTURE IN DIMENSION THREE
}

\author{
DANIEL LE ${ }^{\oplus 1}$, BAO V. LE HUNG ${ }^{2}$, \\ BRANDON LEVIN $^{3}$ and STEFANO MORRA ${ }^{4}$ \\ ${ }^{1}$ Department of Mathematics, University of Toronto, 40 St. George Street, \\ Toronto, ON M5S 2E4, Canada; \\ email: le@math.toronto.edu \\ ${ }^{2}$ Department of Mathematics, Northwestern University, 2033 Sheridan Road, \\ Evanston, IL 60208, USA; \\ email: lhvietbao@googlemail.com \\ ${ }^{3}$ Department of Mathematics, University of Arizona, 617 N. Santa Rita Avenue, P.O. Box \\ 210089, Tucson, Arizona 85721, USA; \\ email: bwlevin@math.arizona.edu \\ ${ }^{4}$ Université Paris 8, Laboratoire Analyse, Géométrie et Applications, Université Sorbonne Paris \\ Nord, CNRS, UMR 7539, F-93430, Villetaneuse, France; \\ email: morra@math.univ-paris13.fr
}

Received 31 August 2016; accepted 28 January 2020

\begin{abstract}
We prove in generic situations that the lattice in a tame type induced by the completed cohomology of a $U(3)$-arithmetic manifold is purely local, that is, only depends on the Galois representation at places above $p$. This is a generalization to $\mathrm{GL}_{3}$ of the lattice conjecture of Breuil. In the process, we also prove the geometric Breuil-Mézard conjecture for (tamely) potentially crystalline deformation rings with Hodge-Tate weights $(2,1,0)$ as well as the Serre weight conjectures of Herzig ['The weight in a Serre-type conjecture for tame $n$-dimensional Galois representations', Duke Math. J. 149(1) (2009), 37-116] over an unramified field extending the results of Le et al. ['Potentially crystalline deformation 3985 rings and Serre weight conjectures: shapes and shadows', Invent. Math. 212(1) (2018), 1-107]. We also prove results in modular representation theory about lattices in Deligne-Lusztig representations for the group $\mathrm{GL}_{3}\left(\mathbb{F}_{q}\right)$.

2010 Mathematics Subject Classification: 11F80 (primary); 11F33, 20C33 (secondary)
\end{abstract}

(C) The Author(s) 2020. This is an Open Access article, distributed under the terms of the Creative Commons Attribution licence (http://creativecommons.org/licenses/by/4.0/), which permits unrestricted re-use, distribution, and reproduction in any medium, provided the original work is properly cited. 


\section{Introduction}

One of the most important developments in the Langlands program in recent years has been the $p$-adic local Langlands correspondence for $\mathrm{GL}_{2}\left(\mathbb{Q}_{p}\right)$. Unfortunately, extending this correspondence even to $\mathrm{GL}_{2}(K)$ has proven to be exceedingly difficult and all evidence suggests that the desired correspondence will be much more complicated. On the other hand, there has been some progress on several avatars of the $p$-adic local Langlands correspondence, namely, (generalized) Serre weight conjectures, geometric Breuil-Mézard conjecture, and Breuil's lattice conjecture. These conjectures inform our understanding of what the sought after $p$-adic correspondence should look like. In this paper, we prove versions of each of these three conjectures for $\mathrm{GL}_{3}(K)$ when $K / \mathbb{Q}_{p}$ is unramified.

1.1. Breuil's lattice conjecture. Motivated by Emerton's local-global compatibility for completed cohomology, [CEG+16] constructs a candidate for one direction of the $p$-adic local Langlands correspondence for $\mathrm{GL}_{n}(K)$. Namely, they associate to any continuous $n$-dimensional $\overline{\mathbb{Q}}_{p}$-representation $\rho$ of $\mathrm{Gal}(\bar{K} / K)$ an admissible Banach space representation $V(\rho)$ of $\mathrm{GL}_{n}(K)$ by patching completed cohomology. However, the construction depends on a choice of global setup, and one expects it to be a deep and difficult problem to show that the correspondence $\rho \mapsto V(\rho)$ is purely local.

In [Bre14], Breuil formulates a conjecture on lattices in tame types cut out by completed cohomology of Shimura curves which is closely related to the local nature of $V(\rho)$. This conjecture was proven subsequently in the groundbreaking work of Emerton-Gee-Savitt [EGS15]. Our first main theorem is a generalization of Breuil's conjecture to three-dimensional Galois representations and the completed cohomology of $U(3)$-arithmetic manifolds.

Specifically, let $p$ be a prime, $F / F^{+}$a CM extension (i.e. $F$ a totally imaginary quadratic extension of a totally real field $F^{+}$) unramified everywhere, and $r: G_{F} \rightarrow \mathrm{GL}_{3}\left(\overline{\mathbb{Q}}_{p}\right)$ a Galois representation. Let $\lambda$ be the Hecke eigensystem corresponding to $r$, which appears in the cohomology of a $U(3)$-arithmetic manifold. Choose a place $v \mid p$ of $F^{+}$which splits in $F$, and let $\widetilde{H}$ be the integral $p$-adically completed cohomology with infinite level at $v$. One expects completed cohomology to realize a global $p$-adic Langlands correspondence generalizing the case of $\mathrm{GL}_{2 / \mathbb{Q}}$. That is, by letting $\widetilde{v}$ denote a place of $F$ above $v$ and by letting $G_{F_{\widetilde{v}}}$ be the absolute Galois group of $F_{\widetilde{v}}$, the $\mathrm{GL}_{3}\left(F_{\widetilde{v}}\right)$ representation on the Hecke eigenspace $\widetilde{H}[\lambda]$ corresponds to $\left.r\right|_{G_{F}}$ via a hypothetical $p$-adic local Langlands correspondence (when the level outside $v$ is chosen minimally). In particular, the globally constructed object $\widetilde{H}[\lambda]$ should depend only on $\left.r\right|_{G_{\widetilde{v}}}$. 
Suppose that $r$ is tamely potentially crystalline with Hodge-Tate weights (2, $1,0)$ at each place above $p$. For simplicity, in Section 1, we suppose that $r$ is unramified away from $p$, although our results hold if $r$ is minimally split ramified. Assume that each place $v \mid p$ in $F^{+}$splits in $F$ and fix a place $\widetilde{v} \mid v$ for all $v \mid p$ in $F^{+}$. Let $\sigma(\tau)$ be the tame type corresponding to the WeilDeligne representations associated with $\left.r\right|_{G_{\widetilde{v}}}$ for all $v \mid p$ under the inertial local Langlands correspondence. Throughout Section 1, the tame type $\sigma(\tau)$ is assumed to be sufficiently generic. If $r$ is modular, then by classical local-global compatibility, $\widetilde{H}[\lambda][1 / p]$ contains $\sigma(\tau)$ with multiplicity one.

THEOREM 1.1.1 (Breuil's conjecture, cf. Theorem 5.3.5). Assume that $p$ is unramified in $\mathrm{F}^{+}$and that $\bar{r}$ satisfies Taylor-Wiles hypotheses and is semisimple at places above $p$. Assume that the level of $\widetilde{H}$ outside $p$ is minimal with respect to $r$. Then, the lattice

$$
\sigma(\tau)^{0}:=\sigma(\tau) \cap \widetilde{H}[\lambda] \subset \sigma(\tau)
$$

depends only on the collection $\left\{\left.r\right|_{G_{F_{v}}}\right\}_{v \mid p}$.

Let $\bar{H}$ be the $\bmod p$ reduction of $\widetilde{H}$ so that $\bar{H}$ is the $\bmod p$ cohomology with infinite level at places above $p$ of a $U(3)$-arithmetic manifold. We prove the following 'mod $p$ multiplicity one' result (cf. Theorem 5.3.4).

THEOREM 1.1.2 (Theorem 5.3.4). Keep the assumptions of Theorem 1.1.1. Let $\sigma(\tau)^{\sigma}$ be a lattice in $\sigma(\tau)$ such that its mod p reduction $\bar{\sigma}(\tau)^{\sigma}$ has an irreducible upper alcove cosocle. Then $\operatorname{Hom}_{K_{p}}\left(\bar{\sigma}(\tau)^{\sigma}, \bar{H}[\lambda]\right)$ is one-dimensional.

These theorems should be compared to [EGS15, Theorems 8.2.1 and 10.2.1] in dimension two. In the special case where $p$ is split in $F^{+}$and $\bar{r}$ is irreducible above $p$, both theorems were proven by the first author in [Le18].

The main ingredients used in [EGS15] are the Taylor-Wiles patching method, the geometric Breuil-Mézard conjecture for potentially Barsotti-Tate Galois deformation rings (building on the work of [Bre14]), and a classification of lattices in tame types (extending [Bre14, BP13]). When we began this project, only the first of these tools was available in the case of $\mathrm{GL}_{3}$. The analogue of potentially Barsotti-Tate Galois deformation rings are potentially crystalline deformation rings with Hodge-Tate weights $(2,1,0)$. In [LLHLM18], we develop a technique for computing these Galois deformation rings when the descent data is tame and sufficiently generic. We discuss in Section 1.2 the geometric Breuil-Mézard conjecture for these rings. The representationtheoretic results are discussed in Section 1.3. 
Several key differences which distinguish our situation from [EGS15] are worth mentioning. Breuil's conjecture for $\mathrm{GL}_{2}$ gave an explicit description of the lattice $\sigma(\tau)^{0}$ in terms of the Dieudonné module of $\left.r\right|_{G_{F_{\tilde{v}}}}$. We prove abstractly that $\sigma(\tau)^{0}$ is 'purely local' (Theorems 5.2.3 and 5.3.5) but without giving any explicit description of the lattice. The lattice $\sigma(\tau)^{0}$ is determined by the parameters of the Galois deformation ring but in a complicated way.

Let $\sigma(\tau)^{\sigma}$ be a lattice in $\sigma(\tau)$ whose reduction has irreducible cosocle $\sigma$. To prove Theorem 1.1.2, we show that a certain (minimal) patched module $M_{\infty}\left(\sigma(\tau)^{\sigma}\right)$ is free of rank one over the local Galois deformation ring (with patching variables) $R_{\infty}(\tau)$ (Theorem 5.1.1). In fact, this result is also a key step in our proof of Theorem 1.1.1. In loc. cit., the analogue of this result is Theorem 10.1.1 where they show that the patched module of any lattice with irreducible cosocle is free of rank one. In our situation, it is no longer true that all such patched modules are cyclic. Rather, this is only true when the cosocle $\sigma$ is upper alcove in every embedding. As a consequence of this, one can deduce that the isomorphism class as an $R_{\infty}(\tau)$-module of $M_{\infty}\left(\sigma(\tau)^{\sigma}\right)$ is purely local for any lattice $\sigma(\tau)^{\sigma}$; however, it need not be free.

For the proof that $M_{\infty}\left(\sigma(\tau)^{\sigma}\right)$ is free of rank one when $\sigma$ is upper alcove, we induct on the complexity of the deformation ring. The simplest deformation rings resemble those for $\mathrm{GL}_{2}$ and so we follow the strategy similar to [EGS15]. For the most complicated deformation rings, we build up $M_{\infty}\left(\sigma(\tau)^{\sigma}\right)$ from its subquotients relying on the description of the submodule structure of reduction $\bar{\sigma}(\tau)^{\sigma}$ discussed in Section 1.3 and crucially intersection theory results for components of $\bmod p$ fiber of the Galois deformation ring.

1.2. Serre weight and Breuil-Mézard conjectures. There is an analogous global context for a mod $p$ Galois representation $\bar{r}: G_{F} \rightarrow \mathrm{GL}_{3}(\mathbb{F})$ whose corresponding Hecke eigensystem $\mathfrak{m}$ appears in the $\bmod p$ cohomology with infinite level at $v$ of a $U(3)$-arithmetic manifold. One expects $\bar{H}$ [m] to correspond to $\left.\bar{r}\right|_{G_{\widetilde{v}}}$ via a hypothetical mod $p$ local Langlands correspondence. Furthermore, if we let $W_{v}(\bar{r})$ be the set of Jordan-Hölder factors in the $\mathrm{GL}_{3}\left(\mathcal{O}_{F_{\widetilde{v}}}\right)$-socle of $\bar{H}[\mathrm{~m}]$ and $\left.\bar{r}\right|_{G_{F_{\tilde{v}}}}$ is tamely ramified, [Her09, GHS18] predict that $W_{v}(\bar{r})=W^{?}\left(\left.\bar{r}\right|_{I_{F_{v}}}\right)$, where $I_{F_{\widetilde{v}}}$ denotes the inertia subgroup of $G_{F_{\widetilde{v}}}$ and $W^{?}\left(\left.\bar{r}\right|_{I_{F_{\tilde{v}}}}\right.$ ) is a set which is explicitly defined in terms of $\left.\bar{r}\right|_{I_{F_{\tilde{v}}}}$ (Strictly speaking, the Hecke eigenspace $\bar{H}[\mathfrak{m}]$ is a smooth representation of $G\left(F_{v}^{+}\right)$, where $G_{/ F_{v}^{+}}$ is a reductive group having a reductive model $\mathcal{G}_{v}$ over $\mathcal{O}_{F_{v}^{+}}$together with an isomorphism $\iota_{\widetilde{v}}: \mathcal{G}_{v}\left(\mathcal{O}_{F_{v}^{+}}\right) \stackrel{\sim}{\rightarrow} \mathrm{GL}_{3}\left(\mathcal{O}_{F_{\tilde{v}}}\right)$.). We have the following version of the weight part of Serre's conjecture (cf. Theorem 5.3.3). 
THEOREM 1.2.1 (The weight part of Serre's conjecture). Assume that $p$ is unramified in $F^{+}$and that $\bar{r}$ satisfies Taylor-Wiles hypotheses, has split ramification, and is semisimple and sufficiently generic at places above $p$. Then $W_{v}(\bar{r})=W^{?}\left(\left.\bar{r}\right|_{I_{\widetilde{v}}}\right)$.

In [LLHLM18], we prove this theorem with the additional assumption that $p$ is split in $F^{+}$. The strategy is to show the numerical Breuil-Mézard conjecture for the simplest deformation rings (where the shape has length of at least 2) using [LLHLM18, Section 6.2]. The key new tool is a more conceptual and robust combinatorial technique for computing the intersection between the predicted weights $W^{?}\left(\left.\bar{r}\right|_{I_{F_{v}}}\right)$ and the Jordan-Hölder factors of a type and which is developed in Section 2. This allows us to inductively prove that all predicted weights are modular.

Using a patching functor which is constructed globally, we show that the generic fibers of tamely potentially crystalline deformation rings of Hodge-Tate weight $(2,1,0)$ are connected for $\bar{\rho}$ generic and deduce the full numerical BreuilMézard conjecture for these Galois deformation rings. Using the numerical formulation, we prove the following geometric version of the Breuil-Mézard conjecture (cf. [EG14], Proposition 3.6.1).

THEOREM 1.2.2 (Proposition 3.6.1). Assume that $\left.\bar{r}\right|_{G_{F_{v}}}$ is semisimple and sufficiently generic. There is a unique assignment $\sigma \mapsto \mathfrak{p}(\sigma)$ taking Serre weights $\sigma \in W^{?}\left(\left.\bar{r}\right|_{G_{F_{v}}}\right)$ to prime ideals in the unrestricted framed deformation ring $R_{\left.\bar{r}\right|_{G_{F}}}^{\square}$ such that the special fiber Spec $\left(\bar{R}_{\left.\bar{r}\right|_{F_{\tilde{v}}}}^{\tau}\right)$ of the potentially crystalline framed deformation ring $R_{\left.\bar{r}\right|_{F_{\tilde{v}}}}^{\tau}$ of Hodge-Tate weight $(2,1,0)$ and tame type $\tau$ is the reduced underlying subscheme of

$$
\bigcup_{\sigma \in W^{?}\left(\left.\bar{r}\right|_{I_{\widetilde{v}}}\right) \cap \mathrm{JH}(\overline{\sigma(\tau)})} \operatorname{Spec}\left(R_{\left.\bar{r}\right|_{G_{F}}}^{\square} / \mathfrak{p}(\sigma)\right) .
$$

Moreover, this is compatible with any patching functor.

1.3. Representation theory results. In order to deduce Breuil's lattice conjecture from the Breuil-Mézard conjecture, we need (and prove) new results on integral structures in Deligne-Lusztig representations, which may be of independent interest. The main theorem (Theorem 4.1.9) is a classification of integral lattices with irreducible cosocle in tame types, by means of an extension graph, which plays a key role in the proofs of Theorems 1.1.1 and 1.1.2.

We now briefly describe the extension graph. In Section 2.1, we introduce a graph on the set of $p$-regular Serre weights (with fixed central character), with 
vertices corresponding to $p$-regular Serre weights and adjacency between vertices described in a combinatorially explicit way. We then show in Lemma 4.2.6 that two vertices are adjacent if and only if the corresponding Serre weights have a nontrivial $\mathrm{GL}_{3}\left(\mathbb{F}_{q}\right)$-extension between them, justifying the terminology. This gives a natural notion of graph distance $\mathrm{d}_{\mathrm{gph}}$ between two $p$-regular Serre weights. Theorem 4.1.9 states the following.

THEOREM 1.3.1 (Theorem 4.1.9). Assume that $R$ is a sufficiently generic Deligne-Lusztig representation of $\mathrm{GL}_{3}\left(\mathbb{F}_{q}\right)$. (In particular, the Jordan-Hölder factors of $\bar{R}$ occur with multiplicity one.) If $\sigma$ is a Jordan-Hölder factor of $\bar{R}$, let $R^{\sigma}$ be the unique lattice up to homothety with cosocle $\sigma$. If $\sigma, \sigma^{\prime} \in \mathrm{JH}(\bar{R})$ and that $\mathrm{d}_{\mathrm{gph}}\left(\sigma, \sigma^{\prime}\right)=d$, then:

(1) $\sigma^{\prime}$ is a direct summand of the dth layer of the cosocle filtration of $\bar{R}^{\sigma}$;

(2) if $\sigma^{\prime \prime} \in \mathrm{JH}(\bar{R})$ is such that $\mathrm{d}_{\mathrm{gph}}\left(\sigma, \sigma^{\prime \prime}\right)=d+1$ and $\mathrm{d}_{\mathrm{gph}}\left(\sigma^{\prime}, \sigma^{\prime \prime}\right)=1$, then $\bar{R}^{\sigma}$ has a subquotient which is isomorphic to the unique nonsplit extension of $\sigma^{\prime}$ by $\sigma^{\prime \prime}$; and

(3) if $R^{\sigma^{\prime}} \subseteq R^{\sigma}$ is a saturated inclusion of lattices, then $p^{d} R^{\sigma} \subseteq R^{\sigma^{\prime}}$ is also a saturated inclusion of lattices.

The argument is involved, using a mixture of local and global techniques, but we can distinguish two main steps in its proof. In the first step (Sections 4.2.3 and 4.2.4), we prove the first two items of Theorem 1.3.1 in the case when $\sigma$ is a lower alcove weight of defect zero (cf. Definition 2.3.8 and Theorem 4.2.16). The proof uses methods from the modular representation theory of algebraic groups, embedding $\bar{R}^{\sigma}$ in a Weyl module with non- $p$-restricted highest weights. The key local argument is a careful study of the restriction of algebraic representations to rational points (Proposition 4.2.10), which lets us constrain the submodule structure of (part of) the $\mathrm{GL}_{3}\left(\mathbb{F}_{q}\right)$-restriction of an algebraic Weyl module in terms of the extension graph. This method does not work for all weights $\sigma \in$ $\mathrm{JH}(\bar{R})$, as the corresponding lattices will not always have simple socle and thus cannot be embedded into a Weyl module.

In the second step (Section 4.3), we reduce the theorem for the remaining lattices to the case treated in the first step. We relate the first two items and the last item of Theorem 1.3.1. The last item, a statement in characteristic zero, is amenable to an inductive analysis. First, we show that for a fixed weight $\sigma \in \operatorname{JH}(\bar{R})$, item (3) of Theorem 1.3.1 actually implies the other two items (cf. Proposition 4.3.16). This crucially uses Theorem 1.3.1(3) in the case $d=1$ (cf. Proposition 4.3.7), which is proved using the computation of deformation 
rings in [LLHLM18], combinatorics of Section 3, and the Kisin-Taylor-Wiles patching method. This argument follows the suggestion in [EGS15, Section B.2] that tamely potentially crystalline deformation rings strongly reflect aspects of local representation theory through global patching constructions.

Next, we show that the first two items of Theorem 1.3.1 applied to $R$ and its dual in the case of lower alcove defect zero weights imply Theorem 1.3.1(3) in the case of lower alcove defect zero weights. From this starting point, an inductive argument proves Theorem 1.3.1(3), thus concluding the proof of Theorem 1.3.1.

1.4. Notation. If $F$ is any field, we write $G_{F} \stackrel{\text { def }}{=} \operatorname{Gal}(\bar{F} / F)$ for the absolute Galois group, where $\bar{F}$ is a separable closure of $F$. If $F$ is a number field and $v$ is a prime of $F$, we write $G_{F_{v}}$ for the decomposition group at $v$ and $I_{F_{v}}$ for the inertia subgroup of $G_{F_{v}}$. If $F$ is a $p$-adic field, we write $I_{F}$ to denote the inertia subgroup of $G_{F}$.

We fix once and for all an algebraic closure $\overline{\mathbb{Q}}$ of $\mathbb{Q}$. All number fields are considered as subfield of our fixed $\overline{\mathbb{Q}}$. Similarly, if $\ell \in \mathbb{Q}$ is a prime, we fix algebraic closures $\overline{\mathbb{Q}}_{\ell}$ as well as embeddings $\overline{\mathbb{Q}} \hookrightarrow \overline{\mathbb{Q}}_{\ell}$. All finite extensions of $\mathbb{Q}_{\ell}$ will thus be considered as subfields in $\overline{\mathbb{Q}}_{\ell}$. Moreover, the residue field of $\overline{\mathbb{Q}}_{\ell}$ is denoted by $\overline{\mathbb{F}}_{\ell}$.

Let $p>3$ be a prime. For $f>0$, we let $K$ be the unramified extension of $\mathbb{Q}_{p}$ of degree $f$. We write $k$ for its residue field (of cardinality $q=p^{f}$ ) and $\mathcal{O}_{K}=W(k)$ for its ring of integers. For $r \geqslant 1$, we set $e_{r} \stackrel{\text { def }}{=} p^{f r}-1$ and fix a compatible system of roots $\pi_{r} \stackrel{\text { def }}{=}(-p)^{\frac{1}{e_{r}}} \in \bar{K}$. We write $e$ for $e_{1}$ and $\pi$ for $\pi_{1}$. Define the extension $L=K(\pi)$ and set $\Delta_{0} \stackrel{\text { def }}{=} \operatorname{Gal}(L / K)$. The choice of the root $\pi$ lets us define a character

$$
\begin{aligned}
\widetilde{\omega}_{\pi}: \Delta_{0} & \rightarrow W(k)^{\times} \\
g & \mapsto \frac{g(\pi)}{\pi}
\end{aligned}
$$

whose associated residual character is denoted by $\omega_{\pi}$. In particular, for $f=1, \omega_{\pi}$ is the mod $p$ cyclotomic character, which will be simply denoted by $\omega$. If $F_{w} / \mathbb{Q}_{p}$ is a finite extension and $W_{F_{w}} \leqslant G_{F_{w}}$ denotes the Weil group, we normalize Artin's reciprocity map $\operatorname{Art}_{F_{w}}: F_{w}^{\times} \rightarrow W_{F_{w}}^{\text {ab }}$ in such a way that uniformizers are sent to geometric Frobenius elements.

Let $E \subset \overline{\mathbb{Q}}_{p}$ be a finite extension of $\mathbb{Q}_{p}$, which will be our coefficient field. We write $\mathcal{O}$ for its ring of integers, fix an uniformizer $\varpi \in \mathcal{O}$, and let $\mathfrak{m}_{E}=(\varpi)$. We write $\mathbb{F} \stackrel{\text { def }}{=} \mathcal{O} / \mathfrak{m}_{E}$ for its residue field. We will always assume that $E$ is 
sufficiently large. In particular, we will assume that any embedding $\sigma: K \hookrightarrow \overline{\mathbb{Q}}_{p}$ factors through $E \subset \overline{\mathbb{Q}}_{p}$.

We fix an embedding $\sigma_{0}: K \hookrightarrow E$. The embedding $\sigma_{0}$ induces maps $\mathcal{O}_{K} \hookrightarrow \mathcal{O}$ and $k \hookrightarrow \mathbb{F}$; we will abuse notation and denote all these by $\sigma_{0}$. We let $\varphi$ denote the $p$ th power Frobenius on $k$ and set $\sigma_{i} \stackrel{\text { def }}{=} \sigma_{0} \circ \varphi^{-i}$. The choice of $\sigma_{0}$ gives $\omega_{f} \stackrel{\text { def }}{=} \sigma_{0} \circ \widetilde{\omega}_{\pi}: I_{K} \rightarrow \mathcal{O}^{\times}$, a fundamental character of niveau $f$. We fix once and for all a sequence $\underline{p} \stackrel{\text { def }}{=}\left(p_{n}\right)_{n \in \mathbb{N}}$ where $p_{n} \in \overline{\mathbb{Q}}_{p}$ satisfies $p_{n+1}^{p}=p_{n}$ and $p_{0}=-p$. We let $K_{\infty} \stackrel{\text { def }}{=} \bigcup_{n \in \mathbb{N}} K\left(p_{n}\right)$ and $G_{K_{\infty}} \stackrel{\text { def }}{=} \operatorname{Gal}\left(\overline{\mathbb{Q}}_{p} / K_{\infty}\right)$.

Let $\rho: G_{K} \rightarrow \mathrm{GL}_{n}(E)$ be a $p$-adic, de Rham Galois representation. For $\sigma$ : $K \hookrightarrow E \subset \overline{\mathbb{Q}}_{p}$, we define $\operatorname{HT}_{\sigma}(\rho)$ to be the multiset of $\sigma$-labeled Hodge-Tate weights of $\rho$, that is, the set of integers $i$ such that $\operatorname{dim}_{E}\left(\rho \otimes_{\sigma, K} \mathbb{C}_{p}(-i)\right)^{G_{K}} \neq 0$ (with the usual notation for Tate twists). In particular, the cyclotomic character has Hodge-Tate weights 1 for all embedding $\sigma \hookrightarrow E$. For $\mu=\left(\mu_{j}\right)_{j} \in X^{*}(\underline{T})$, we say that $\rho$ has Hodge-Tate weighs $\mu$ if

$$
\operatorname{HT}_{\sigma_{j}}(\rho)=\left\{\mu_{1, j}, \mu_{2, j}, \ldots, \mu_{n, j}\right\} .
$$

The inertial type of $\rho$ is the isomorphism class of $\left.\operatorname{WD}(\rho)\right|_{I_{K}}$, where $\operatorname{WD}(\rho)$ is the Weil-Deligne representation attached to $\rho$ as in [CDT99], Appendix B.1 (in particular, $\rho \mapsto \mathrm{WD}(\rho)$ is covariant). An inertial type is a morphism $\tau: I_{K} \rightarrow$ $\mathrm{GL}_{n}(E)$ with open kernel and which extends to the Weil group $W_{K}$ of $G_{K}$. We say that $\rho$ has type $(\mu, \tau)$ if $\rho$ has Hodge-Tate weights $\mu$ and inertial type given by (the isomorphism class of) $\tau$.

Let $G \stackrel{\text { def }}{=} \mathrm{GL}_{3 / \mathbb{F}}$, denote by $T \subseteq G$ the torus of diagonal matrices, and write $W$ (respectively $W_{a}$, respectively $\widetilde{W}$ ) for the Weyl group (respectively the affine Weyl group, respectively the extended affine Weyl group) of $G$. We let $X^{*}(T)$ denote the group of characters of $T$, which we identify with $\mathbb{Z}^{3}$ in the usual way. Let $R$ (respectively $R^{\vee}$ ) denote the set of roots (respectively coroots) of $G$ and $\Lambda_{R} \subseteq X^{*}(T)$ the root lattice. We then have

$$
W_{a}=\Lambda_{R} \rtimes W(G), \quad \widetilde{W}=X^{*}(T) \rtimes W(G) .
$$

Let $\varepsilon_{1}^{\prime}$ and $\varepsilon_{2}^{\prime}$ be $(1,0,0)$ and $(0,0,-1)$, respectively. Let $G^{\text {der }}$ be $\mathrm{SL}_{3 / \mathbb{F}}$ with torus $T^{\text {der }}$. Let $\Lambda_{W}=X^{*}\left(T^{\text {der }}\right)$ denote the weight lattice for $G^{\text {der }}$. Let $X^{0}(T)$ denote the kernel of the restriction map $X^{*}(T) \rightarrow \Lambda_{W}$. We write $\varepsilon_{1}$ and $\varepsilon_{2}$ for the images of $\varepsilon_{1}^{\prime}$ and $\varepsilon_{2}^{\prime}$ in $\Lambda_{W}$, respectively. We define in a similar fashion the various Weyl groups $W^{\text {der }}, W_{a}^{\text {der }}, \widetilde{W}^{\text {der }}$ for $G^{\text {der }}$. Note that we have canonical isomorphisms $W \cong W^{\text {der }}$ and $W_{a} \cong W_{a}^{\text {der }}$.

Let $\mathcal{S}$ be a finite set. For each $\widetilde{v} \in \mathcal{S}$, let $F_{\widetilde{v}}$ be an unramified extension of $\mathbb{Q}_{p}$ of degree $f_{\widetilde{v}}$, and let $k_{\widetilde{v}}$ be the residue field of $F_{\widetilde{v}}$. Let $\mathcal{J}$ be the set of ring 
homomorphisms $\prod_{\widetilde{v} \in \mathcal{S}} k_{\widetilde{v}} \rightarrow \mathbb{F}$. If we fix an embedding $\sigma_{\widetilde{v}, 0}: k_{\widetilde{v}} \hookrightarrow \mathbb{F}$ and set $\sigma_{\widetilde{v}, i}$ to be $\sigma_{\widetilde{v}, 0} \circ \varphi^{-i}$, then $\mathcal{J}$ is naturally identified with the set of pairs $\left(\widetilde{v}, i_{\widetilde{v}}\right)$ with $\tilde{v} \in \mathcal{S}$ and $i_{\widetilde{v}} \in \mathbb{Z} / f_{\widetilde{v}}$. In applications, $\mathcal{S}$ will be a finite set of places dividing $p$ of a number field $F$. Sometimes, $\mathcal{S}$ will have cardinality one, in which case we might drop the subscripts from $f_{\widetilde{v}}$ and $k_{\widetilde{v}}$ and denote the single unramified extension $F_{\widetilde{v}}$ of $\mathbb{Q}_{p}$ by $K$.

Let $\underline{G}_{0}$ be the algebraic group $\prod_{\tilde{v} \in \mathcal{S}} \operatorname{Res}_{k_{\tilde{v}} / \mathbb{F}_{p}} \mathrm{GL}_{3}$ with $\underline{T}_{0}$ the diagonal torus and center $\underline{Z}_{0}$. Let $\underline{G}$ be the base change $\underline{G}_{0} \times_{\mathbb{F}_{p}} \mathbb{F}$, and similarly define $\underline{T}$ and $\underline{Z}$. There is a natural isomorphism $\underline{G} \cong \prod_{i \in \mathcal{J}} \mathrm{GL}_{3 / \mathbb{F}}$. One has similar isomorphisms for $\underline{T}, \underline{Z}, X^{*}(\underline{T}), \underline{R}, \underline{R}^{\vee}$, where $\underline{R}$ (respectively $\underline{R}^{\vee}$ ) denotes the set of roots (respectively coroots) of $\underline{G}$. If $\mu \in X^{*}(\underline{T})$, then we correspondingly write $\mu=$ $\sum_{i \in \mathcal{J}} \mu_{i}=\sum_{\widetilde{v} \in \mathcal{S}} \mu_{\widetilde{v}}$. We use similar notation for similar decompositions. Again, we identify $X^{*}(\underline{T})$ with $\left(\mathbb{Z}^{3}\right)^{\mathcal{J}}$ in the usual way and let $\varepsilon_{1, i}^{\prime}$ and $\varepsilon_{2, i}^{\prime}$ be $(1,0,0)$ and $(0,0,-1)$, respectively, in the $i$ th coordinate and 0 otherwise. Let $\underline{R}^{+} \subseteq \underline{R}$ (respectively $\underline{R}^{\vee,+} \subseteq \underline{R}^{\vee}$ ) be the subset of positive roots (respectively coroots) of $\underline{G}$ with respect to the upper triangular Borel in each embedding. We define dominant (co)characters with respect to these choices. Let $X_{+}^{*}(\underline{T)}$ be the set of dominant weights. We denote by $X_{1}\left(\underline{T)} \subset X_{+}^{*}(\underline{T})\right.$ the subset of weights $\lambda \in$ $X_{+}^{*}(\underline{T})$ satisfying $0 \leqslant\left\langle\lambda, \alpha^{\vee}\right\rangle \leqslant p-1$ for all simple roots $\alpha \in \underline{R}^{+}$. We call $X_{1}(\underline{T})$ the set of $p$-restricted weights. Let $\eta_{i}^{\prime} \in X^{*}(\underline{T})$ be $(1,0,-1)$ in the $i$ th coordinate and 0 otherwise, and let $\eta^{\prime}$ be $\sum_{i \in \mathcal{J}} \eta_{i}^{\prime} \in X^{*}(\underline{T})$. Let $\eta_{i} \in X^{*}(\underline{T})$ be $(2,1,0)$ in the $i$ th coordinate and 0 otherwise, and let $\eta$ be $\sum_{i \in \mathcal{J}} \eta_{i} \in X^{*}(\underline{T})$. Then $\eta$ is a lift of the half-sum of the positive roots of $G$.

Let $\underline{W}$ be the Weyl group of $\underline{G}$ with longest element $\bar{w}_{0}$. Let $\underline{W}_{a}$ and $\underline{\widetilde{W}}$ be the affine Weyl group and extended affine Weyl group, respectively, of $\underline{G}$. Let $\underline{\Lambda}_{R} \subset$ $X^{*}(\underline{T})$ denote the root lattice of $\underline{G}$. As above, we have identifications $\underline{W} \cong W^{\mathcal{J}}$, $\underline{W}_{a} \cong W_{a}^{\mathcal{J}}, \underline{\widetilde{W}} \cong \widetilde{W}^{\mathcal{J}}$ and isomorphisms analogous to (1.1).

The Weyl groups $\underline{W}, \underline{\widetilde{W}}, \underline{W}_{a}$ act naturally on $X^{*}(\underline{T})$. The image of $\lambda \in X^{*}(\underline{T})$ via the injection $X^{*}\left(\underline{T) \hookrightarrow} \underline{\widetilde{W}}\right.$ is denoted by $t_{\lambda}$. Our convention is that the dot action is always a $p$-dot action, that is, $t_{\lambda} w \cdot \mu=t_{p \lambda} w(\mu+\eta)-\eta$.

Recall that for $(\alpha, n) \in \underline{R}^{+} \times \mathbb{Z}$, we have the root hyperplane $H_{\alpha, n} \stackrel{\text { def }}{=}\{\lambda$ : $\left.\left\langle\lambda+\eta, \alpha^{\vee}\right\rangle=n p\right\}$. An alcove (or sometimes $p$-alcove) is a connected component of the complement $X^{*}(\underline{T}) \otimes_{\mathbb{Z}} \mathbb{R} \backslash\left(\bigcup_{(\alpha, n)} H_{\alpha, n}\right)$. We say that an alcove $\underline{C}$ is $p$ restricted if $0<\left\langle\lambda+\eta, \alpha^{\vee}\right\rangle<p$ for all simple roots $\alpha \in \underline{R}^{+}$and $\lambda \in \underline{C}$. If $\underline{C}_{0} \subset X^{*}(\underline{T}) \otimes_{\mathbb{Z}} \mathbb{R}$ denotes the dominant base alcove, we let

$$
\underline{\widetilde{W}}^{+} \stackrel{\text { def }}{=}\left\{\widetilde{w} \in \underline{\widetilde{W}}: \widetilde{w} \cdot \underline{C}_{0} \text { is dominant }\right\}
$$

and

$$
\underline{\widetilde{W}}_{1}^{+} \stackrel{\text { def }}{=}\left\{\widetilde{w} \in \underline{\widetilde{W}}^{+}: \widetilde{w} \cdot \underline{C}_{0} \text { is } p \text {-restricted }\right\} .
$$


Let $\widetilde{w}_{h}=\left(\widetilde{w}_{h, i}\right) \in \widetilde{W}_{1}^{+}$be the element $w_{0} t_{-\eta}$. The discussion in this paragraph also applies for $G$, from which we define the dominant base alcove $C_{0}, \widetilde{W}^{+}$, and $\widetilde{W}_{1}^{+}$for $G$.

There is a Frobenius action on $X^{*}(\underline{T})$, denoted by $\pi$ and an induced action of $\pi$ on $\underline{W}$ defined by $\pi(w)(\pi(\lambda))=\pi(w(\lambda))$. If $\lambda \in X^{*}(\underline{T})$, then $\pi(\lambda)_{\widetilde{v}, i_{\widetilde{v}}}=\lambda_{\widetilde{v}, i_{\tilde{v}}-1}$ under the standard identification and similarly $\pi(s)_{\widetilde{v}, i_{\widetilde{v}}}=s_{\widetilde{v}, i_{\widetilde{v}}-1}$ for an element $s$ of one of the Weyl groups above.

Let $\underline{G}_{0}^{\text {der }}$ be $\prod_{\widetilde{v} \in \mathcal{S}} \operatorname{Res}_{k_{\tilde{v}} / \mathbb{F} p} \mathrm{SL}_{3}$ with torus $\underline{T}_{0}^{\text {der }}$. Let $\underline{G}^{\text {der }}$ be the base change $\underline{G}_{0}^{\text {der }} \times_{\mathbb{F}_{p}} \mathbb{F}$, and similarly define $\underline{T}^{\text {der }}$. Let $\underline{\Lambda}_{W}=X^{*}\left(\underline{T}^{\text {der }}\right)$ denote the weight lattice for $\underline{G}^{\text {der }}$. Let $X^{0}(\underline{T})$ denote the kernel of the restriction map $X^{*}\left(\underline{T)} \rightarrow \underline{\Lambda}_{W}\right.$. We write $\varepsilon_{1, i}$ and $\varepsilon_{2, i}$ for the images of $\varepsilon_{1, i}^{\prime}$ and $\varepsilon_{2, i}^{\prime}$, respectively. We define in a similar fashion the various Weyl groups $\underline{W}^{\mathrm{der}}, \underline{W}_{a}^{\mathrm{der}}, \underline{\widetilde{W}}^{\mathrm{der}}, \underline{\widetilde{W}}^{\mathrm{der},+}, \underline{\widetilde{W}}_{1}^{\mathrm{der},+}$ for $\underline{G}^{\mathrm{der}}$ (and $\widetilde{W}^{\text {der,+}}, \widetilde{W}_{1}^{\text {der,+ }}$ for $G$ ), with analogous product decompositions.

Let $\alpha, \beta, \gamma^{+}$denote the generators for the affine Weyl group $W_{a}$ of $\mathrm{GL}_{3}$ given by reflection over the walls of $C_{0}$ with $\alpha, \beta \in W$, and $\gamma^{+}$is the affine reflection. Note that $\alpha, \beta$ satisfy $\alpha\left(\varepsilon_{2}^{\prime}\right)=\varepsilon_{2}^{\prime}$ and $\beta\left(\varepsilon_{1}^{\prime}\right)=\varepsilon_{1}^{\prime}$.

Let $S_{3}$ denote the symmetric group on $\{1,2,3\}$. We fix an injection $S_{3} \hookrightarrow$ $\mathrm{GL}_{3}(\mathbb{Z})$ sending $s$ to the permutation matrix whose $(k, m)$-entry is $\delta_{k, s(m)}$ and $\delta_{k, s(m)} \in\{0,1\}$ is the Kronecker $\delta$ specialized at $\{k, s(m)\}$. We will abuse notation and simply use $s$ to denote the corresponding permutation matrix. We consider the embedding $X^{*}(T) \hookrightarrow \mathrm{GL}_{3}(\mathbb{F}((v)))$ defined by $\lambda \mapsto v^{\lambda}$ where then $v^{(a, b, c)}$ is the diagonal matrix with entries $v^{a}, v^{b}, v^{c}$, respectively. In this way, we get an embedding $\widetilde{W} \hookrightarrow \mathrm{GL}_{3}(\mathbb{F}((v)))$. Finally, for $m \geqslant 0$ and a collection $\left(B_{j}\right)_{j=0, \ldots, m}$ of square matrices of the same size, we write $\prod_{j=0}^{m} B_{j}=B_{0} \cdot B_{1} \cdots B_{m}$.

Let $V$ be a representation of a finite group $\Gamma$ over an $E$-vector space. We write $\mathrm{JH}(\bar{V})$ to denote the set of Jordan-Hölder factors of the mod $\varpi$-reduction of an $\mathcal{O}$-lattice in $V$. This set is independent of the choice of the lattice.

If $R$ is a ring, we let $\operatorname{Irr}(\operatorname{Spec}(R))$ denote the set of minimal primes of $R$.

\section{Extension graph}

In this section, we give a description of the extension graph of generic irreducible representations of the group $\mathrm{G} \stackrel{\text { def }}{=} \underline{G}_{0}\left(\mathbb{F}_{p}\right)$ and describe the constituents of the mod $p$ reduction of generic Deligne-Lusztig representations of G. This also gives a description of the set $W^{?}(\bar{\rho})$ of predicted Serre weights defined by Herzig [Her09]. In Section 3, we use these descriptions to prove the Serre weight conjectures. The distance in the extension graph also plays an important role in computing the cosocle filtration for the reductions of lattices in tame types in Section 4.3. 
2.1. Definition and properties of the extension graph. The surjection $X^{*}(T) \rightarrow \Lambda_{W}$ identifies $p$-restricted alcoves of $\mathrm{GL}_{3}$ and $\mathrm{SL}_{3}$. Let $C_{0}$ and $C_{1}$ denote the $p$-restricted lower and upper alcoves, respectively, of the weight space of $\mathrm{GL}_{3}$ so that $\mathcal{A} \stackrel{\text { def }}{=}\left\{C_{0}, C_{1}\right\}^{\mathcal{J}}$ is naturally identified with the set of $p$-restricted alcoves for $\underline{G}$ (or $\underline{G}^{\text {der }}$ when convenient). Our alcoves are fundamental domains with respect to the dot action, so the base alcove will have a vertex at $-\eta$. For notational convenience, we sometimes write 0 and 1 instead of $C_{0}$ and $C_{1}$ so that $\mathcal{A}$ is identified with $\{0,1\}^{\mathcal{J}}$. We recall some notions from Section 1.4. Let $X_{1}(\underline{T})$ denote the set of $p$-restricted weights of $\underline{G}$ and $\underline{C}_{0} \subseteq X(\underline{T}) \otimes \mathbb{R}$ denote the lowest alcove $\left(C_{0}\right)_{i \in \mathcal{J}}$. We consider $\underline{\widetilde{W}}^{\mathrm{der}}=\underline{\Lambda}_{W} \rtimes \underline{W}$ acting via the $p$-dot action on $\underline{\Lambda}_{W} \otimes \mathbb{R}$. Let $\underline{\widetilde{W}}_{1}^{\mathrm{der},+} \subset \underline{\widetilde{W}}^{\mathrm{der}}$ denote the set of elements which take $\underline{C}_{0}$ to an element of $\mathcal{A}$. There are six elements of $\widetilde{W}_{1}^{\mathrm{der},+}$, namely id, (12) $t_{-\left(\varepsilon_{1}-\varepsilon_{2}\right)}$, (23) $t_{-\left(\varepsilon_{2}-\varepsilon_{1}\right)},(123) t_{-\varepsilon_{2}},(132) t_{-\varepsilon_{1}}$, and (13) $t_{-\left(\varepsilon_{1}+\varepsilon_{2}\right)}$.

Observe that the inclusion $\underline{\Lambda}_{W} \hookrightarrow \widetilde{W}^{\text {der }}$ (respectively $X^{*}(\underline{T}) \hookrightarrow \underline{\widetilde{W}}$ ) induces an isomorphism $\iota^{\text {der }}: \underline{\Lambda}_{W} / \underline{\Lambda}_{R} \stackrel{\sim}{\rightarrow} \underline{\widetilde{W}}^{\text {der }} / \underline{W}_{a}^{\text {der }}$ (respectively $\iota: X^{*}(\underline{T}) / \underline{\Lambda}_{R}(\cong$ $\left.\left.X^{*}(\underline{Z})\right) \stackrel{\sim}{\rightarrow} \underline{\widetilde{W}} / \underline{W}_{a}\right)$. Let $\mathcal{P}^{\text {der }} \subset \underline{\Lambda}_{W} \times \underline{\widetilde{W}}_{1}^{\text {der, }+}$ be the subset of pairs $(\omega, \widetilde{w})$ with $\iota^{\text {der }}\left(-\pi^{-1}(\omega)+\underline{\Lambda}_{R}\right)=\widetilde{w} \underline{W}_{a}^{\text {der }}$. We similarly define $\mathcal{P} \subset X^{*}(\underline{T}) \times \underline{W}_{1}^{+}$. Note that restriction gives a natural surjection $\mathcal{P} \rightarrow \mathcal{P}^{\text {der }}$.

LEMMA 2.1.1. The map

$$
\begin{aligned}
\beta: \mathcal{P}^{\text {der }} & \rightarrow \underline{\Lambda}_{W} \times \mathcal{A} \\
(\omega, \widetilde{w}) & \mapsto\left(\omega, \pi(\widetilde{w}) \cdot \underline{C}_{0}\right)
\end{aligned}
$$

is a bijection.

Proof. The map $\beta$ is a bijection because $\iota^{\text {der }}$ is a bijection and $\underline{W}_{a}^{\text {der }}$ acts simply transitively on the set of alcoves for $\underline{G}^{\text {der }}$.

Let $\mu$ be an element of $\underline{C}_{0} /(p-\pi) X^{0}(\underline{T})$. We will often have some lift of $\mu$ in $X^{*}(\underline{T})$ in mind, but what we write will not depend on the choice of this lift. We define a map

$$
\begin{aligned}
\mathcal{P}^{\text {der }} & \rightarrow X^{*}(\underline{T}) /(p-\pi) X^{0}(\underline{T}) \\
(\omega, \tilde{w}) & \mapsto \widetilde{w}^{\prime} \cdot\left(\mu-\eta+\omega^{\prime}\right),
\end{aligned}
$$

where $\left(\omega^{\prime}, \widetilde{w}^{\prime}\right) \in \mathcal{P}$ is a lift of $(\omega, \widetilde{w})$. The map (2.1) does not depend on the choice of lift. Then we define

$$
\mathfrak{T r}_{\mu}^{\prime}: \underline{\Lambda}_{W} \times \mathcal{A} \rightarrow X^{*}(\underline{T}) /(p-\pi) X^{0}(\underline{T})
$$

to be the composition of $\beta^{-1}$ with (2.1). 
Definition 2.1.2. We say that a weight $\lambda \in X^{*}(\underline{T})$ is $p$-regular if $\langle\lambda+\eta$, $\left.\alpha^{\vee}\right\rangle \notin p \mathbb{Z}$ for all positive coroots $\alpha^{\vee} \in \underline{R}^{\vee,+}$.

Note that a $p$-regular element belongs to a unique alcove.

Define $\underline{\Lambda}_{W}^{\mu}$ to be the set

$$
\left\{\omega \in \underline{\Lambda}_{W}: \omega+\mu-\eta \in \underline{C}_{0}\right\}
$$

(taking the image of $\mu-\eta$ in $\underline{\Lambda}_{W}$ ). Let $\mathfrak{T r}_{\mu}$ be the restriction of $\mathfrak{T r}_{\mu}^{\prime}$ to $\underline{\Lambda}_{W}^{\mu} \times \mathcal{A}$. We establish some basic properties of $\mathfrak{T r}_{\mu}$.

Proposition 2.1.3. (1) (Any lift of) $\mathfrak{T r}_{\mu}(\omega, a)$ in $X^{*}(\underline{T})$ is $p$-regular and is in alcove $\pi^{-1}(a)$.

(2) The map $\mathfrak{T r}_{\mu}$ is injective.

Proof. For (1), suppose that $\left(\omega^{\prime}, \widetilde{w}^{\prime}\right) \in \mathcal{P}$ is a lift of $\beta^{-1}(\omega, a)$. Then $\mu-\eta+\omega^{\prime}$ is $p$-regular in alcove $\underline{C}_{0}$ by definition of $\underline{\Lambda}_{W}^{\mu}$ so that $\mathfrak{T r}_{\mu}(\omega, a)=\widetilde{w}^{\prime} \cdot\left(\mu-\eta+\omega^{\prime}\right)$ is $p$-regular in alcove $\pi^{-1}(a)=\widetilde{w} \cdot \underline{C}_{0}$.

For (2), suppose that $\mathfrak{T r}_{\mu}(\omega, a)=\mathfrak{T r}_{\mu}(\nu, b)$. Let $\left(\omega^{\prime}, \widetilde{w}^{\prime}\right)$ and $\left(v^{\prime}, \widetilde{x}^{\prime}\right) \in \mathcal{P}$ be lifts of $\beta^{-1}(\omega, a)$ and $\beta^{-1}(\nu, b)$, respectively. Then $\widetilde{w}^{\prime} \cdot\left(\mu-\eta+\omega^{\prime}\right) \equiv \tilde{x}^{\prime} \cdot(\mu-$ $\left.\eta+v^{\prime}\right)\left(\bmod (p-\pi) X^{0}(\underline{T})\right)$, from which we conclude from the definition of $\mathcal{P}^{\text {der }}$ that $\left.\left.\omega^{\prime}\right|_{\underline{Z}} \equiv v^{\prime}\right|_{\underline{Z}}\left(\bmod 3 X^{*}(\underline{Z})\right)$. Combining this with the fact that $a=b$ from (1) and using $\iota$, we see that $\widetilde{w}^{\prime} \equiv \widetilde{x}^{\prime}\left(\bmod X^{0}(\underline{T})\right)$. This implies that $\omega=v$.

Proposition 2.1.4. Let $\mu \in X^{*}(\underline{T})$ be a character. We have

$$
\begin{aligned}
& \mathfrak{T r}_{\mu}\left(\underline{\Lambda}_{W}^{\mu} \times \mathcal{A}\right) \\
& \quad=\left\{\begin{array}{ll}
\lambda \in X_{1}(\underline{T}) /(p-\pi) X^{0}(\underline{T}): & \lambda \text { is p-regular and } \\
& \left.(\lambda-\mu+\eta)\right|_{\underline{Z}} \in(p-\pi) X^{*}(\underline{Z})
\end{array}\right\}
\end{aligned}
$$

Proof. The inclusion of the left-hand side of (2.2) in the right follows from Proposition 2.1.3(1) and the formula for $\mathfrak{T r}_{\mu}$. We show now the reverse inclusion. Let $\lambda$ be an element in the right-hand side of (2.2). Then we let

- $a \in \mathcal{A}$ denote the unique alcove that contains $\pi(\lambda)$;

- $\omega^{\prime} \in X^{*}(\underline{T})$ be such that $\left.(\lambda-\mu+\eta)\right|_{\underline{Z}}=\left.\left(1-p \pi^{-1}\right) \omega^{\prime}\right|_{\underline{Z}}$;

- $\omega \in \underline{\Lambda}_{W}$ be the image of $\omega^{\prime}$;

- $\left(\omega^{\prime}, \widetilde{w}^{\prime}\right) \in \mathcal{P}$ be a lift of $\beta^{-1}(\omega, a)$; and 
- $v$ be $\lambda-\widetilde{w}^{\prime} \cdot\left(\mu-\eta+\omega^{\prime}\right) \in \underline{\Lambda}_{R}$.

Then $\left(w^{\prime-1}(v)+\omega^{\prime}, \widetilde{w}^{\prime}\right) \in \mathcal{P}$ is a lift of $\beta^{-1}\left(w^{\prime-1}(v)+\omega, a\right)$ so that

$$
\mathfrak{T r}_{\mu}\left(w^{\prime-1}(v)+\omega, a\right)=\lambda
$$

in $X^{*}(\underline{T}) /(p-\pi) X^{0}(\underline{T})$.

Proposition 2.1.5. Let $\lambda-\eta$ be a lift of $\mathfrak{T r}_{\mu}\left(\varepsilon, \underline{C}_{0}\right)$ and $\beta^{-1}\left(\varepsilon, \underline{C}_{0}\right)=(\varepsilon, \widetilde{w})$. Then

$$
\mathfrak{T r}_{\lambda}(v, a)=\mathfrak{T r}_{\mu}\left(w^{-1}(v)+\varepsilon, a\right)
$$

for $(\nu, a) \in \underline{\Lambda}_{W}^{\lambda} \times \mathcal{A}$, where $w \in \underline{W}$ is the image of $\widetilde{w}$.

Proof. Let $\left(v^{\prime}, \widetilde{x}^{\prime}\right) \in \mathcal{P}$ be a lift of $\beta^{-1}(v, a)$. Similarly, let $\left(\varepsilon^{\prime}, \widetilde{w}^{\prime}\right) \in \mathcal{P}$ be a lift of $(\varepsilon, \widetilde{w})$. Then

$$
\begin{aligned}
\mathfrak{T r}_{\lambda}(\nu, a) & =\tilde{x}^{\prime} \cdot\left(\lambda-\eta+v^{\prime}\right) \\
& =\tilde{x}^{\prime} \cdot\left(\widetilde{w}^{\prime} \cdot\left(\mu-\eta+\varepsilon^{\prime}\right)+v^{\prime}\right) \\
& =\tilde{x}^{\prime} \widetilde{w}^{\prime} \cdot\left(\mu-\eta+\varepsilon^{\prime}+w^{-1}\left(v^{\prime}\right)\right) \\
& =\mathfrak{T r}_{\mu}\left(w^{-1}(v)+\varepsilon, a\right) .
\end{aligned}
$$

Recall that a Serre weight is an irreducible $\overline{\mathbb{F}}_{p}$-representation of G. Each Serre weight is obtained by restriction to $G$ from an irreducible algebraic representation of $\underline{G}$ of highest weight $\lambda \in X_{1}(\underline{T})$, and this process gives a bijection between from $X_{1}(\underline{T}) /(p-\pi) X^{0}(\underline{T})$ to the set of Serre weights of $\mathrm{G}$ (as described in [Her09, Theorem 3.10]; cf. also the beginning of Section 4.2). If $\lambda \in X_{1}(\underline{T)}$, we write $F(\lambda)$ for the Serre weight corresponding to $\lambda$. We say that a Serre weight $F$ is $p$-regular if $F \cong F(\lambda)$ where $\lambda \in X_{1}(\underline{T})$ is $p$-regular (cf. Definition 2.1.2). Given $\mu \in \underline{C}_{0}$ and $(\omega, a) \in \underline{\Lambda}_{W}^{\mu} \times \mathcal{A}$, we get a corresponding $p$-regular Serre weight $F\left(\mathfrak{T r}_{\mu}(\omega, a)\right)$. Propositions 2.1.3(2) and 2.1.4 show that $F\left(\mathfrak{T r}_{\mu}(-)\right)$ induces a bijection between the set $\Lambda_{W}^{\mu} \times \mathcal{A}$ and the set of $p$-regular Serre weights of $\mathrm{G}$ with the same central character as $F(\mu-\eta)$.

Definition 2.1.6. We say that $(\omega, a)$ and $(v, b)$ in $\underline{\Lambda}_{W}^{\mu} \times \mathcal{A}$ are adjacent if there exists $j \in \mathcal{J}$ such that both $a_{i}=b_{i}$ and $\omega_{i}=v_{i}$ for $i \in \mathcal{J}$ with $i \neq j$, $a_{j} \neq b_{j}$, and

$$
\omega_{j}-v_{j} \in\left\{0, \pm\left(\varepsilon_{1, j}-\varepsilon_{2, j}\right), \pm \varepsilon_{1, j}, \pm \varepsilon_{2, j}\right\} .
$$

If $F$ and $F^{\prime}$ are $p$-regular Serre weights, we say that they are adjacent if we can write $F=F\left(\mathfrak{T r}_{\mu}(\omega, a)\right)$ and $F^{\prime}=F\left(\mathfrak{T r}_{\mu}(\nu, b)\right)$ for some $p$-regular $\mu \in$ $\underline{C}_{0} /(p-\pi) X^{0}(\underline{T})$ and some $(\omega, a)$ and $(\nu, b)$ which are adjacent in $\underline{\Lambda}_{W}^{\mu} \times \mathcal{A}$. 
By Proposition 2.1.5, this definition does not depend on the choice of the lowest alcove weight $\mu \in \underline{C}_{0}$. Indeed, if $F=F\left(\mathfrak{T r}_{\mu}(\omega, a)\right), F^{\prime}=F\left(\mathfrak{T r}_{\mu}(\nu, b)\right)$ are adjacent $p$-regular Serre weights and $\lambda \in C_{0}$ such that $F(\lambda-\eta)$ and $F(\mu-\eta)$ have the same central character, then $F\left(\mathfrak{T r}_{\lambda}(w(\omega-\varepsilon), a)\right)=F\left(\mathfrak{T r}_{\mu}(\omega, a)\right)$ and $F\left(\mathfrak{T r}_{\lambda}(w(\nu-\varepsilon), b)\right)=F\left(\mathfrak{T r}_{\mu}(\nu, b)\right)$ for some $w \in \underline{W}$ and $\varepsilon \in \underline{\Lambda}_{W}$, and $(w(\omega-$ $\varepsilon), a),(w(v-\varepsilon), b)$ are again adjacent since the set in (2.3) is $\underline{W}$-invariant.

REMARK 2.1.7. Geometrically, $\underline{\Lambda}_{W}^{\mu}$ is the intersection of $\underline{\Lambda}_{W}$ with a translate of a $p$-alcove, and two pairs $(\omega, a)$ and $(v, b)$ are adjacent if and only if $\omega$ and $v$ are either equal or neighbors (that is, differ by a $\underline{W}$-conjugate of a fundamental weight) and either $\omega \neq v$ and $a$ and $b$ have different labels in the unique component where $\omega$ and $v$ differ or $\omega=v$ and $a$ and $b$ differ in exactly one component. Note that for any $(\omega, a) \in \underline{\Lambda}_{W}^{\mu}$ with all its neighbors in $\underline{\Lambda}_{W}^{\mu}$, there are $7^{\# \mathcal{J}}$ adjacent vertices.

Definition 2.1.6 endows $\underline{\Lambda}_{W}^{\mu} \times \mathcal{A}$ with a graph structure with edges given by adjacency. By the above description, this graph is connected and thus it is endowed with a metric. By Proposition 2.1.4, any $\mu \in \underline{C}_{0}$ thus endows the set of $p$-regular Serre weights with the same central character as $F(\mu-\eta)$ with a metric. By Proposition 2.1.5, the metric on a fixed set is independent of the choice of $\mu$.

DEFINITION 2.1.8. Given $p$-regular Serre weights $F, F^{\prime}$ with the same central character, we denote their distance by $\mathrm{d}_{\mathrm{gph}}\left(F, F^{\prime}\right)$. In particular, $F$ and $F^{\prime}$ are adjacent if and only if $\mathrm{d}_{\mathrm{gph}}\left(F, F^{\prime}\right)=1$.

We conclude this section by showing the relation between $p$-regular Serre weights and G-extensions. We start by recalling the following definition.

Definition 2.1.9. Let $\lambda \in X^{*}(T)$ be a weight. We say that $\lambda$ lies $n$-deep in its alcove if, for all $\alpha^{\vee} \in R^{\vee,+}$, there exist integers $m_{\alpha} \in \mathbb{Z}$ such that $p m_{\alpha}+n<$ $\left\langle\lambda+\eta, \alpha^{\vee}\right\rangle<p\left(m_{\alpha}+1\right)-n$. We have the analogous definition for weights in $X^{*}(\underline{T})$.

For instance, a dominant weight $\lambda \in X^{*}(\underline{T})$ lies $n$-deep in alcove $\underline{C}_{0}$ if $n<$ $\left\langle\lambda+\eta, \alpha^{\vee}\right\rangle<p-n$ for all $i=0, \ldots, f-1$ and all positive coroots $\alpha^{\vee} \in \underline{R}^{\vee,+}$.

A Serre weight $F$ is said to be $n$-deep if we can write $F \cong F(\mu)$ for some $\mu \in X_{1}(\underline{T})$ which is $n$-deep. We call the graph on the $p$-regular Serre weights defined by adjacency the extension graph. The terminology is justified by the following theorem. 
THEOREM 2.1.10. Let $F, F^{\prime}$ be Serre weights which are both 6-deep. Then $\operatorname{Ext}_{\mathrm{G}}^{1}\left(F, F^{\prime}\right) \neq 0$ if and only if $F$ and $F^{\prime}$ are adjacent.

Proof. This is Lemma 4.2.6, whose proof only uses modular representation theory and does not rely on any results from other sections.

REMARK 2.1.11. We partition the set of vertices $(\omega, a)$ into two sets, according to the class $\Sigma_{i} a_{i}$ mod 2 (by interpreting $a_{i} \in\{0,1\}$ ). This makes the extension graph into a bipartite graph.

2.2. Types and Serre weights. Now suppose that $\mathcal{S}$ contains exactly one element $\widetilde{v}$, and let $K$ be $F_{\widetilde{v}}$. An inertial type is a representation $\tau: I_{K} \rightarrow \mathrm{GL}_{3}(E)$ with open kernel which extends to the Weil group of $K$. An inertial type is tame if it factors through the tame inertial quotient. All our tame inertial types are defined over $\mathcal{O}$ and we use $\bar{\tau}: I_{K} \rightarrow \mathrm{GL}_{3}(\mathbb{F})$ to denote the reduction to the residue field.

Tame inertial types have a combinatorial description which we will now recall (cf. [Her09, (6.15)] or [GHS18, Definition 8.2.2]). Let $(w, \mu) \in \underline{W} \times X^{*}(\underline{T})$. As in [Her09, (4.1)] or the paragraph preceding [GHS18, Definition 10.1.12], for any $(\nu, \sigma) \in X^{*}(\underline{T}) \rtimes \underline{W}$, define

$$
{ }^{(v, \sigma)}(w, \mu)=\left(\sigma w \pi(\sigma)^{-1}, \sigma(\mu)+p v-\sigma w \pi(\sigma)^{-1} \pi(\nu)\right)
$$

and we write $(w, \mu) \sim\left(w^{\prime}, \mu^{\prime}\right)$ if there exists $(\nu, \sigma)$ such that ${ }^{(v, \sigma)}(w, \mu)=\left(w^{\prime}\right.$, $\left.\mu^{\prime}\right)$. The following describes all isomorphism classes of tame inertial types for $K$.

DEFINITION 2.2.1. Define an inertial type $\tau(w, \mu): I_{K} \rightarrow \mathrm{GL}_{3}(\mathcal{O})$ as follows: If $w=\left(s_{0}, \ldots, s_{f-1}\right)$, then set $s_{\tau}=s_{0} s_{f-1} s_{f-2} \cdots s_{1} \in W$ and $\alpha \in X^{*}(\underline{T})$ such that $\boldsymbol{\alpha}_{0}=\mu_{0}$ and $\boldsymbol{\alpha}_{j}=s_{1}^{-1} s_{2}^{-1} \ldots s_{j}^{-1}\left(\mu_{j}\right)$ for $1 \leqslant j \leqslant f-1$. Let $r$ denote the order of $s_{\tau}$, and set $f^{\prime}=f r$. Then,

$$
\tau(w, \mu) \stackrel{\text { def }}{=} \bigoplus_{1 \leqslant i \leqslant 3} \omega_{f^{\prime}}^{\sum_{0 \leqslant k \leqslant r-1} \mathbf{a}_{s_{\tau}(i)}^{(0)} p^{f k}}
$$

where $\mathbf{a}^{(0)}:=\sum_{j=0}^{f-1} \boldsymbol{\alpha}_{j} p^{j} \in \mathbb{Z}^{3}$. Note that $(w, \mu) \sim\left(\left(s_{\tau}, 1, \ldots, 1\right), \boldsymbol{\alpha}\right)$ and $\tau(w$, $\mu) \cong \tau\left(\left(s_{\tau}, 1, \ldots, 1\right), \boldsymbol{\alpha}\right)$ by construction.

DEFINITION 2.2.2. Let $\tau$ be a tame inertial type.

(1) We say that $\tau$ is regular if the characters appearing in the right-hand side of (2.5) are pairwise distinct. 
(2) We say that $\tau$ is $n$-generic if there is an isomorphism $\tau \cong \tau(s, \lambda+\eta)$ for some $s \in \underline{W}$ and $\lambda \in X_{+}^{*}(\underline{T})$ which is $n$-deep in alcove $\underline{C}_{0}$.

(3) We say that $\bar{\rho}: G_{K} \rightarrow \mathrm{GL}_{3}(\mathbb{F})$ is $n$-generic if $\left.\bar{\rho}^{\mathrm{ss}}\right|_{I_{K}} \cong \bar{\tau}$ for a tame inertial type $\tau$ which is $n$-generic.

(4) A lowest alcove presentation of $\tau$ is a pair $(s, \mu) \in \underline{W} \times X^{*}(\underline{T})$ where $\mu \in \underline{C}_{0}$ such that $\tau \cong \tau(s, \mu+\eta)$ (which by definition exists exactly when $\tau$ is 0 -generic).

REMARK 2.2.3. The notion of genericity given in [LLHLM18, Definition 2.1] is slightly different than Definition 2.2.2(2). In particular, an inertial type which is $n$-generic in the sense of Definition 2.2.2(2) is a fortiori $n$-generic in the sense of [LLHLM18]. Furthermore, our notion of genericity differs from that of [GHS18, Definition 10.1.12] by a shift by $\eta$.

REMARK 2.2.4. If $\tau$ is 1 -generic, then $\tau$ is regular.

Now let $\mathcal{S}$ have arbitrary (finite) cardinality rather than one. For $s \in \underline{W}$ and $\mu \in X^{*}(\underline{T})$, we can define $s_{\widetilde{v}}$ and $\mu_{\widetilde{v}}$ in the evident way. Then we set $\tau_{\mathcal{S}}(s, \mu)$ to be the collection $\left(\tau_{\widetilde{v}}\left(s_{\widetilde{v}}, \mu_{\widetilde{v}}\right)\right)_{\widetilde{v} \in \mathcal{S}}$.

The notions of regular and $n$-generic are extended in the evident way to a collection $\tau_{\mathcal{S}}(s, \mu)$ of tame inertial types. Similarly, there is an evident notion of $n$-generic for a collection $\bar{\rho}_{\mathcal{S}}$ of Galois representations $\bar{\rho}_{\widetilde{v}}: G_{F_{v}} \rightarrow \mathrm{GL}_{3}(\mathbb{F})$ for $\tilde{v} \in \mathcal{S}$.

We now introduce the Deligne-Lusztig representations which are relevant for this paper. See also [GHS18, Section 9.2] and [LLHL19, Section 2.2], though we note that our context is slightly more general than [LLHL19, Section 2.2] since $\mathcal{S}$ may have size greater than one. Let $(s, \mu) \in \underline{W} \times X^{*}(\underline{T})$ be a good pair [LLHL19, Section 2.2]. Following [GHS18, Propositions 9.2.1 and 9.2.2], we can attach to $(s, \mu)$ a genuine Deligne-Lusztig representation $R_{s}(\mu)$ of $\mathrm{G} \stackrel{\text { def }}{=}$ $\underline{G}_{0}\left(\mathbb{F}_{p}\right)$ defined over $E$ (taking $E$ to be sufficiently large). Note that $R_{s}(\mu)$ is denoted as $R(s, \mu)$ in [GHS18].

DEFINITION 2.2.5. Let $(s, \mu) \in \underline{W} \times X^{*}(\underline{T})$ be a good pair and let $n \geqslant 0$. We say that $R_{s}(\mu)$ is $n$-generic if there exists an isomorphism $R_{s}(\mu) \cong R_{s^{\prime}}\left(\mu^{\prime}\right)$ where $\mu^{\prime}-\eta$ is $n$-deep in alcove $\underline{C}_{0}$ (note that the evident generalization of [LLHL19, Lemma 2.2.3] implies that $\left(s^{\prime}, \mu^{\prime}\right)$ is good). By [GHS18, Proposition 9.2.1] and the evident generalization of [LLHL19, Proposition 2.2.4], when $n \geqslant 0$, then $\tau_{\mathcal{S}}(s, \mu)$ is $n$-generic (cf. Definition 2.2.2(2)) if and only if $R_{s}(\mu)$ is $n$-generic. 
By inflation, we consider $R_{s}(\mu)$ as a smooth $\prod_{\widetilde{v} \in \mathcal{S}} \mathrm{GL}_{3}\left(\mathcal{O}_{F_{\widetilde{v}}}\right)$-representation. We recall some basic results on $R_{s}(\mu)$. Let $\sigma\left(\tau_{\widetilde{v}}\right)$ be a smooth $\mathrm{GL}_{3}\left(\mathcal{O}_{F_{\tilde{v}}}\right)$ representation associated with $\tau_{\widetilde{v}}$ as in [CEG+16, Theorem 3.7], and let $\sigma\left(\tau_{\mathcal{S}}\right)$ be the $\prod_{\widetilde{v} \in \mathcal{S}} \mathrm{GL}_{3}\left(\mathcal{O}_{F_{\tilde{v}}}\right)$-representation $\bigotimes_{\widetilde{v} \in \mathcal{S}} \sigma\left(\tau_{\widetilde{v}}\right)$.

Proposition 2.2.6. Let $\mu-\eta \in X^{*}(\underline{T})$ be 1-deep in alcove $\underline{C}_{0}$. Then:

(1) [DL76, Theorem 6.8] $R_{s}(\mu)$ is irreducible.

(2) [LLHL19, Corollary 2.3.5] $\sigma\left(\tau_{\mathcal{S}}\right)$ can be taken to be $R_{s}(\mu)$.

We conclude this section with some background on Serre weights associated with semisimple Galois representations. Let $\bar{\tau}_{\mathcal{S}}$ be a collection of tame representations $\bar{\tau}_{\widetilde{v}}: I_{F_{\widetilde{v}}} \rightarrow \mathrm{GL}_{3}(\mathbb{F})$ which extend to $G_{F_{\tilde{v}}}$. By [GHS18, Proposition 9.2.1], one attaches to $\bar{\tau}_{\widetilde{v}}$ an $E$-valued representation $V\left(\bar{\tau}_{\widetilde{v}}\right)$ of $\mathrm{GL}_{3}\left(k_{\widetilde{v}}\right)$. Let $V\left(\bar{\tau}_{\mathcal{S}}\right)$ be the G-representation $\bigotimes_{\widetilde{v} \in \mathcal{S}} V\left(\bar{\tau}_{\widetilde{v}}\right)$. When $\bar{\tau}_{\mathcal{S}}$ is $\bar{\tau}_{\mathcal{S}}(s, \mu)$ for a good pair $(s, \mu), V\left(\bar{\tau}_{\mathcal{S}}\right)$ is isomorphic to $R_{s}(\mu)$ [GHS18, Proposition 9.2.3]. We write $\left.\bar{\rho}_{\mathcal{S}}\right|_{I_{F_{\mathcal{S}}}}$ for the restriction to inertia of the collection $\bar{\rho}_{\mathcal{S}}$.

Let $\widetilde{w}_{h} \stackrel{\text { def }}{=} w_{0} t_{-\eta} \in \underline{\widetilde{W}}$. Recall the self-bijection $\mathcal{R}$ on $p$-regular Serre weights defined in [GHS18, Section 9.2]:

$$
\mathcal{R}(F(\lambda)) \stackrel{\text { def }}{=} F\left(\widetilde{w}_{h} \cdot \lambda\right)
$$

Definition 2.2.7 [GHS18, Definition 9.2.5]. Let $\bar{\rho}_{\mathcal{S}}$ be a collection of 2generic semisimple Galois representations $\bar{\rho}_{\widetilde{v}}: G_{F_{\widetilde{v}}} \rightarrow \mathrm{GL}_{3}(\mathbb{F})$. The set of predicted weights for $\bar{\rho}_{\mathcal{S}}$ is defined to be

$$
W^{?}\left(\bar{\rho}_{\mathcal{S}}\right) \stackrel{\text { def }}{=}\left\{\mathcal{R}(F): F \in \mathrm{JH}\left(\overline{V\left(\left.\bar{\rho}_{\mathcal{S}}\right|_{I_{\mathcal{S}}}\right)}\right)\right\} .
$$

If $\tau_{\mathcal{S}}$ is a collection of tame inertial types $\tau_{\widetilde{v}}: I_{F_{\widetilde{v}}} \rightarrow \mathrm{GL}_{3}(\mathcal{O})$, we furthermore define

$$
W^{?}\left(\bar{\rho}_{\mathcal{S}}, \tau_{\mathcal{S}}\right) \stackrel{\text { def }}{=} W^{?}\left(\bar{\rho}_{\mathcal{S}}\right) \cap \mathrm{JH}\left(\overline{\sigma\left(\tau_{\mathcal{S}}\right)}\right)
$$

REMARK 2.2.8. The condition that $\left.\bar{\rho}_{\mathcal{S}}\right|_{I_{F_{\mathcal{S}}}}$ is 2-generic is to ensure that the elements of $\mathrm{JH}\left(\overline{V\left(\left.\bar{\rho}_{\mathcal{S}}\right|_{I_{F_{\mathcal{S}}}}\right)}\right)$ are all 0 -deep so that $\mathcal{R}$ is defined (cf. Lemma 4.2.13).

Recall from [GHS18, Definition 7.1.3] that there is a subset $W_{\text {obv }}(\bar{\rho}) \subseteq W^{?}(\bar{\rho})$ of obvious Serre weights of $\bar{\rho}$. The set $W_{\text {obv }}\left(\bar{\rho}_{\mathcal{S}}\right)$ is defined in the evident way. 
2.3. Combinatorics of types and Serre weights. Recall the notation $\bar{\rho}_{\mathcal{S}}$ from the previous section. We will always assume that $\bar{\rho}_{\widetilde{v}}$ is 2-generic and semisimple for all $\widetilde{v} \in \mathcal{S}$ in what follows. The following proposition describes $W_{\text {obv }}\left(\bar{\rho}_{\mathcal{S}}\right)$ in terms of the extension graph.

Proposition 2.3.1. Assume that $\bar{\rho}_{\mathcal{S}}$ is a collection of semisimple Galois representations. Suppose that $\left.\bar{\rho}_{\mathcal{S}}\right|_{I_{F_{\mathcal{S}}}} \cong \bar{\tau}_{\mathcal{S}}(s, \lambda)$, where $\lambda-\eta$ is 3-deep in $\underline{C}_{0}$. Then $W_{\mathrm{obv}}\left(\bar{\rho}_{\mathcal{S}}\right)$ is the set

$$
\left\{F\left(\mathfrak{T r}_{\lambda}\left(s(\omega), \pi(\widetilde{w}) \cdot \underline{C}_{0}\right)\right): \widetilde{w}=w t_{-\pi^{-1} \omega} \in \underline{\widetilde{W}}_{1}^{+, \mathrm{der}}\right\} .
$$

Proof. This follows from [LLHL19, Corollary 2.2.13 and (2.6)].

DeFINITION 2.3.2. Define

$$
\Sigma_{0} \stackrel{\text { def }}{=}\left\{\begin{array}{c}
\left(\varepsilon_{1}+\varepsilon_{2}, 0\right),\left(\varepsilon_{1}-\varepsilon_{2}, 0\right),\left(\varepsilon_{2}-\varepsilon_{1}, 0\right) \\
(0,1),\left(\varepsilon_{1}, 1\right),\left(\varepsilon_{2}, 1\right) \\
(0,0),\left(\varepsilon_{1}, 0\right),\left(\varepsilon_{2}, 0\right)
\end{array}\right\} .
$$

Define $\Sigma \stackrel{\text { def }}{=} \Sigma_{0}^{\mathcal{J}} \subset \underline{\Lambda}_{W} \times \mathcal{A}$.

The set of predicted weights $W^{?}\left(\bar{\rho}_{\mathcal{S}}\right)$ and the Jordan-Hölder factors of a Deligne-Lusztig representation $R_{s}(\mu)$ will be described in terms of the 'triangle' $\Sigma_{0}$ inside $\Lambda_{W} \times\left\{C_{0}, C_{1}\right\}$ (see Figure 1).

DEFINITION 2.3.3. Define the following subsets of $\Sigma_{0}$ :

$$
\begin{aligned}
& \Sigma_{0}^{\text {obv }} \stackrel{\text { def }}{=}\left\{\begin{array}{c}
\left(\varepsilon_{1}+\varepsilon_{2}, 0\right),\left(\varepsilon_{1}-\varepsilon_{2}, 0\right),\left(\varepsilon_{2}-\varepsilon_{1}, 0\right) \\
(0,1),\left(\varepsilon_{1}, 1\right),\left(\varepsilon_{2}, 1\right)
\end{array}\right\}, \\
& \Sigma_{0}^{\text {inn }} \stackrel{\text { def }}{=} \Sigma_{0} \backslash \Sigma_{0}^{\text {obv }} .
\end{aligned}
$$

We say that $(\omega, a) \in \Sigma$ is obvious (respectively inner) in component $i$ if ( $\omega_{i}$, $\left.a_{i}\right) \in \Sigma_{0}^{\text {obv }}$ (respectively $\left.\left(\omega_{i}, a_{i}\right) \in \Sigma_{0}^{\text {inn }}\right)$.

We define an involution $r$ of $\underline{\Lambda}_{W}^{\mu} \times \mathcal{A}$ by $r(\omega, a)=\left(\omega, a^{\prime}\right)$ with $a_{i} \neq a_{i}^{\prime}$ for all $i \in \mathcal{J}$.

Proposition 2.3.4. Let $\mu \in X^{*}(\underline{T})$. Suppose that $V\left(\left.\bar{\rho}_{\mathcal{S}}\right|_{I_{F_{\mathcal{S}}}}\right)=R_{s}(\mu+v)$ where $v \in \underline{\Lambda}_{R}$ and $\mu+v-\eta$ is 3-deep in alcove $\underline{C}_{0}$. Then $W^{\text {? }}\left(\bar{\rho}_{\mathcal{S}}\right)$ is $F\left(\mathfrak{T r}_{\mu}\left(t_{\nu} \operatorname{sr}(\bar{\Sigma})\right)\right)$. 


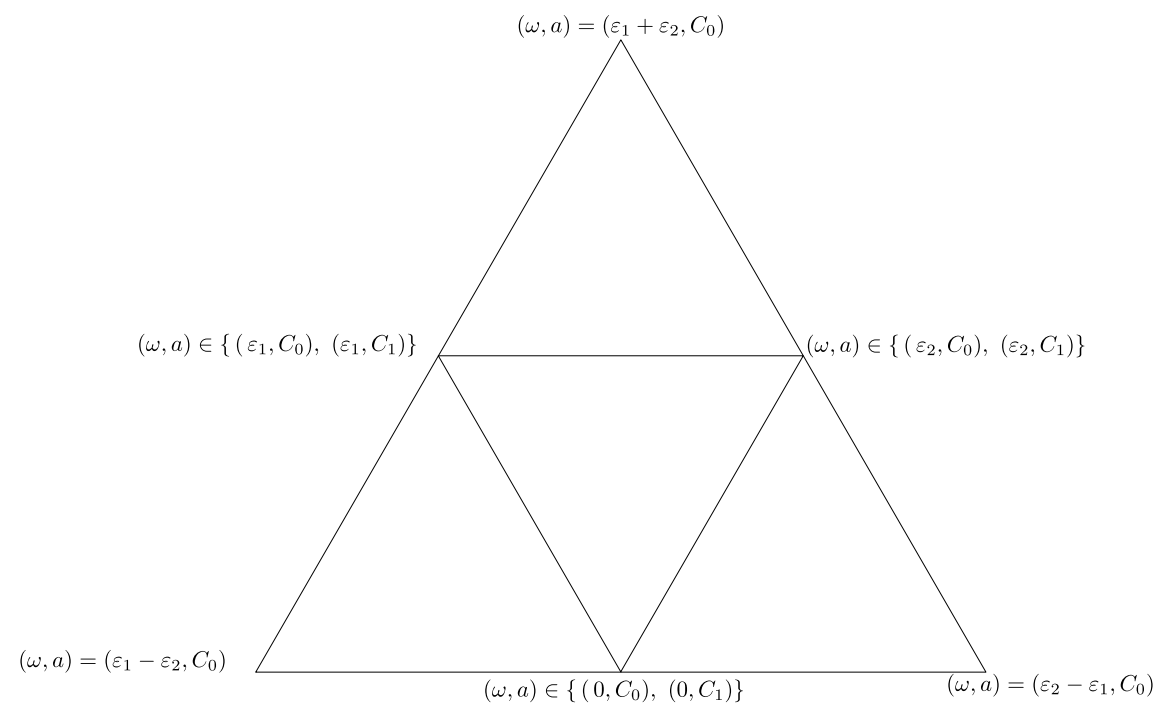

Figure 1. The set $\Sigma_{0}$.

Proof. By Proposition 2.3.1, $W_{\text {obv }}\left(\bar{\rho}_{\mathcal{S}}\right)$ is given by

$$
\left\{F\left(\mathfrak{T r}_{\mu+v}\left(s(\omega), \pi(\widetilde{w}) \cdot \underline{C}_{0}\right)\right): \widetilde{w}=w t_{-\pi^{-1} \omega} \in \underline{W}_{1}^{+, \text {der }}\right\} .
$$

Since $v \in \underline{\Lambda}_{R}$, we have $\mathfrak{T r}_{\mu+v}\left(s(\omega), \pi(\widetilde{w}) \cdot \underline{C}_{0}\right)=\mathfrak{T r}_{\mu}\left(s(\omega)+v, \pi(\widetilde{w}) \cdot \underline{C}_{0}\right)$. By the generalization of Jantzen's formula in [Her09, Appendix, Theorem 3.4], the reduction of $R_{s}(\mu+v)$ is given in terms of reductions of Weyl modules. The fact that $\mu+v-\eta$ is 3-deep implies that these Weyl modules decompose in a generic pattern. Hence, $W^{?}\left(\bar{\rho}_{\mathcal{S}}\right)$ is given by [GHS18, Proposition 10.1.8], which is

$$
F\left(\left\{\mathfrak{T r}_{\mu}\left(t_{v} s(\omega), a\right) \mid \tilde{w}=w t_{-\pi^{-1} \omega} \in \underline{W}_{1}^{+, \text {der }}, \pi(\widetilde{w}) \cdot \underline{C}_{0} \uparrow a\right\}\right) .
$$

This set is precisely $F\left(\mathfrak{T r}_{\mu}\left(t_{v} \operatorname{sr}(\Sigma)\right)\right)$.

PRoposition 2.3.5. If $v \in X^{*}(\underline{T})$ such that $v+\eta \in \underline{\Lambda}_{R}$ and $\mu+v-\eta$ is 3-deep in alcove $\underline{C}_{0}$, then $\mathrm{JH}\left(\overline{R_{s}(\mu+v)}\right)=F\left(\mathfrak{T r}_{\mu}\left(t_{v} s(\Sigma)\right)\right)$.

Proof. Since $t_{1} \circ \mathcal{R} \circ \mathfrak{T r}_{\mu}=\mathfrak{T r}_{\mu} \circ r, F\left(\mathfrak{T r}_{\mu}(\omega, a)\right) \in \mathrm{JH}\left(\overline{R_{s}(\lambda-\underline{1})}\right)$ if and only if $F\left(\mathfrak{T r}_{\mu}(r(\omega, a))\right) \in \mathcal{R}\left(\mathrm{JH}\left(\overline{R_{s}(\lambda)}\right)\right)=W^{?}\left(\bar{\rho}_{\mathcal{S}}\right)$ where $V\left(\left.\bar{\rho}\right|_{I_{\mathcal{S}}}\right)=R_{s}(\lambda)$. The result then follows from Proposition 2.3.4. 
DEFinition 2.3.6. For any $\widetilde{w} \in \underline{W}$, we set $R_{\widetilde{w}}(\mu) \stackrel{\text { def }}{=} R_{w}(\mu+\widetilde{w}(0))$, where $w \in \underline{W}$ is the projection of $\widetilde{w}$.

There is an action of $\underline{\widetilde{W}}$ on $\underline{\Lambda}_{W} \times \mathcal{A}$ where it acts through $\underline{\widetilde{W}}^{\text {der }}$ on just the first factor (via the usual action, not the dot action). By Propositions 2.3.4 and 2.3.5, we have the following.

Proposition 2.3.7. Let $\widetilde{w} \in \underline{W}_{a} t_{\eta}$ and assume that $\mu+\widetilde{w}^{-1}(0)-\eta$ is 3-deep in $\underline{C}_{0}$. Then the set $\mathrm{JH}\left(\overline{R_{\widetilde{w}^{-1}}(\mu)}\right)$ is $F\left(\mathfrak{T r}_{\mu}\left(\widetilde{w}^{-1}(\Sigma)\right)\right)$. If $\widetilde{w} \in \underline{W}_{a}$ and $V\left(\left.\bar{\rho}_{\mathcal{S}}\right|_{I_{F_{\mathcal{S}}}}\right)=$ $R_{\widetilde{w}}(\mu)$, then the set $W^{?}\left(\bar{\rho}_{\mathcal{S}}\right)$ is $F\left(\mathfrak{T r}_{\mu}(\widetilde{w}(r(\Sigma)))\right)$.

For the rest of this section, fix a character $\mu$ such that $\mu-\eta$ is 3-deep in $\underline{C}_{0}$ and an element $s \in \underline{W}$.

For $(s \omega, a) \in \underline{\Lambda}_{W}^{\mu} \times \mathcal{A}$, we let $\sigma_{(\omega, a)}^{(s, \mu)}=F\left(\mathfrak{T r}_{\mu}(s \omega, a)\right)$. When the pair $(s, \mu)$ is clear from the context, we will simply write $\sigma_{(\omega, a)}$ instead of $\sigma_{(\omega, a)}^{(s, \mu)}$ to lighten notation. Let $\widetilde{w} \in \underline{W}_{a} t_{\eta}$ such that $s \widetilde{w}^{-1}(\Sigma) \subset \underline{\Lambda}_{W}^{\mu} \times \mathcal{A}$ and $\mu+s \widetilde{w}^{-1}(0)-$ $\eta$ is 3-deep. Then the map

$$
\begin{aligned}
\tilde{w}^{-1}(\Sigma) & \rightarrow \mathrm{JH}\left(\overline{R_{s} \widetilde{w}^{-1}(\mu)}\right) \\
\widetilde{w}^{-1}(\omega, a) & \rightarrow \sigma_{\left(\widetilde{w}^{-1}(\omega), a\right)}^{(s, \mu)}
\end{aligned}
$$

is a bijection by Proposition 2.3.7. Similarly, if $V\left(\left.\bar{\rho}_{\mathcal{S}}\right|_{I_{F_{\mathcal{S}}}}\right)=R_{s}(\mu)$, then the map

$$
\begin{aligned}
r(\Sigma) & \rightarrow W^{?}\left(\bar{\rho}_{\mathcal{S}}\right) \\
r(\omega, a) & \rightarrow \sigma_{r(\omega, a)}^{(s, \mu)}
\end{aligned}
$$

is a bijection.

DEFINITION 2.3.8. We say that $\sigma \in \mathrm{JH}\left(\overline{R_{s \widetilde{w}^{-1}}(\mu)}\right)$ is an outer (respectively inner) weight for $R \stackrel{\text { def }}{=} R_{s \widetilde{w}^{-1}}(\mu)$ in component $i \in \mathcal{J}$ if $\sigma=\sigma_{\left(\widetilde{w}^{-1}(\omega), a\right)}$ with $\left(\omega_{i}, a_{i}\right) \in \Sigma_{0}^{\mathrm{obv}}$ (respectively $\left.\left(\omega_{i}, a_{i}\right) \in \Sigma_{0}^{\mathrm{inn}}\right)$. We define the defect of $\sigma$ with respect to $R$ as

$$
\operatorname{Def}_{R}(\sigma) \stackrel{\text { def }}{=} \#\{i \in \mathcal{J} \text { such that } \sigma \text { is an inner weight of } R \text { in component } i\} .
$$

Similarly, if $V\left(\left.\bar{\rho}_{\mathcal{S}}\right|_{I_{F_{\mathcal{S}}}}\right)=R_{S}(\mu)$, we say that $\sigma \in W^{?}\left(\bar{\rho}_{\mathcal{S}}\right)$ is an obvious (respectively shadow) weight for $\bar{\rho}_{\mathcal{S}}$ in component $i \in \mathcal{J}$ if $\sigma=\sigma_{r(\omega, a)}$ with $\left(\omega_{i}, a_{i}\right) \in \Sigma_{0}^{\text {obv }}$ (respectively $\left.\left(\omega_{i}, a_{i}\right) \in \Sigma_{0}^{\mathrm{inn}}\right)$. We define the defect of $\sigma$ with 
Table 1. The graph $\Sigma_{0}$.

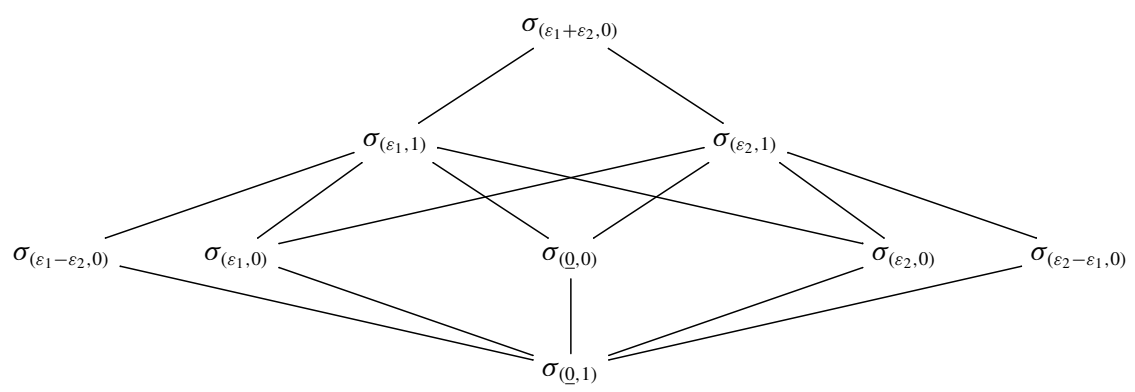

respect to $\bar{\rho}_{\mathcal{S}}$ as

$\operatorname{Def}_{\bar{\rho}_{\mathcal{S}}}(\sigma) \stackrel{\text { def }}{=} \#\left\{i \in \mathcal{J}\right.$ such that $\sigma$ is a shadow weight of $\bar{\rho}_{\mathcal{S}}$ in component $\left.i\right\}$.

We give the set $\Sigma_{0}$ the structure of a graph in Table 1 . We write $\Sigma_{0}^{\mathcal{J}}$ for the corresponding product graph. The above bijections respect the notion of adjacency for $\mathrm{JH}\left(\overline{R_{s} \tilde{w}^{-1}(\mu)}\right), W^{?}\left(\bar{\rho}_{\mathcal{S}}\right)$, and $\Sigma_{0}^{\mathcal{J}}$.

We now describe the intersections of $\mathrm{JH}\left(\overline{R_{s} \tilde{w}^{-1}(\mu)}\right)$ and $W^{?}\left(\bar{\rho}_{\mathcal{S}}\right)$ for $\left.\bar{\rho}_{\mathcal{S}}\right|_{G_{F_{\mathcal{S}}}} \cong$ $\bar{\tau}_{\mathcal{S}}(s, \mu)$ in terms of the action of $\widetilde{W}$ on $\underline{\Lambda}_{W}$ using Proposition 2.3.7. We first introduce a subset of $\underline{\widetilde{W}}$, the admissible set, which appears in Corollary 2.3.11 and again in Section 3.

Recall from Section 1.4 that $\widetilde{W}$ denotes the extended affine Weyl group of $G \stackrel{\text { def }}{=} \mathrm{GL}_{3 / \mathbb{F}}$. The choice of the dominant base alcove endows $\widetilde{W}$ with a Bruhat order which is denoted by $\leqslant$. We also have the natural generalization of the Bruhat order $\leqslant$ on $\underline{\widetilde{W}}$ associated with the choice of the dominant base alcove.

Definition 2.3.9. Let $\lambda \in X^{*}(T)$. We define

$$
\operatorname{Adm}(\lambda)=\left\{\widetilde{w} \in \widetilde{W} \mid \widetilde{w} \leqslant t_{s(\lambda)} \text { for some } s \in W\right\} .
$$

Similarly, for a weight $\mu=\left(\mu_{j}\right)_{j} \in X^{*}(\underline{T})$, define

$$
\operatorname{Adm}(\mu)=\prod_{j} \operatorname{Adm}\left(\mu_{j}\right) \subset \underline{\widetilde{W}} .
$$

REMARK 2.3.10. For combinatorics of weights as in this section, we use the dominant base alcove and the corresponding affine reflection $\gamma^{+}=t_{(1,0,-1)}(13)$ 
as a generator for $W_{a}^{\text {der }}$. When working on the Galois side, as in Section 3, the antidominant base alcove appears naturally, and the analogous set $\operatorname{Adm}^{\vee}(\eta)$ for the antidominant base alcove plays an important role (see Definition 3.1.1 and the discussion before). The affine generator for the antidominant choice of base alcove is $\gamma=(13) t_{(1,0,-1)}$. Note that in [LLHLM18], everything is in terms of antidominant and so what we call $\operatorname{Adm}(\eta)$ there, we denote by $\operatorname{Adm}^{\vee}(\eta)$ here.

For $\widetilde{w} \in \widetilde{W}$, define $\Sigma_{\widetilde{w}} \stackrel{\text { def }}{=} \Sigma_{0} \cap \widetilde{w}\left(r\left(\Sigma_{0}\right)\right)$. Similarly, for $\widetilde{w} \in \underline{\widetilde{W}}$, let $\Sigma_{\widetilde{w}} \stackrel{\text { def }}{=} \Sigma \cap \widetilde{w}(r(\Sigma))$. One can directly compute these sets using Figure 1. For example, using Figure 2, one can check that $\Sigma_{\widetilde{w}}$ is empty if and only if $\widetilde{w}$ is not in $\operatorname{Adm}(\eta) X^{0}(\underline{T})$ (in essence, [LLHL19, Proposition 4.4.2] is a generalization of this fact). Otherwise, see Table 2 for a description of $\Sigma_{\widetilde{w}}$.

COROLlary 2.3.11. Let $\widetilde{w} \in \underline{W}_{a} t_{\eta}$. Assume that both $\mu-\eta$ and $\mu+$ $s \widetilde{w}^{-1}(\underline{0})-\eta$ are 3-deep in $\underline{C}_{0}$. The set $\mathcal{R}\left(\mathrm{JH}\left(\overline{R_{s}(\mu)}\right)\right) \cap \mathrm{JH}\left(\overline{R_{s \widetilde{w}^{-1}}(\mu)}\right)$ is the set of weights $\sigma_{r(\omega, a)}^{(s, \mu)}$ where $(\omega, a) \in \Sigma_{\widetilde{w}^{-1}}$. Similarly, the set $\mathrm{JH}\left(\overline{R_{s}(\mu-\underline{1})}\right) \cap$ $\mathcal{R}\left(\mathrm{JH}\left(\overline{R_{s \widetilde{w}}(\mu-\underline{1})}\right)\right)$ is the set of weights $\sigma_{(\omega, a)}^{(s, \mu)}$ where $(\omega, a) \in \Sigma_{\widetilde{w}}$.

Proof. By Proposition 2.3.7, one can reduce the case where $s=$ id. In that case, (2.6) and (2.7) imply that the set of Serre weights in the intersection $\mathcal{R}\left(\mathrm{JH}\left(\overline{R_{s}(\mu)}\right)\right) \cap \mathrm{JH}\left(\overline{R_{s \widetilde{w}^{-1}}(\mu)}\right)$ is $\mathfrak{T r}_{\mu}\left(\widetilde{w}^{-1}(\Sigma) \cap r(\Sigma)\right)$. We conclude by noting that $\widetilde{w}^{-1}(\Sigma) \cap r(\Sigma)=r\left(\Sigma_{\widetilde{w}^{-1}}\right)$. The statement for $\operatorname{JH}\left(\overline{R_{s}(\mu-\underline{1})}\right) \cap$ $\mathcal{R}\left(\mathrm{JH}\left(\overline{R_{s} \widetilde{w}}(\mu-\underline{1})\right)\right)$ is proved similarly.

Lemma 2.3.12. If $(\omega, a) \in \underline{\Lambda}_{W} \times \mathcal{A} \backslash r(\Sigma)$, there exists $\widetilde{w} \in \underline{W}_{a} t_{\eta}$ such that $(\omega, a) \in \widetilde{w}^{-1}(\Sigma)$ and $\widetilde{w} \notin \operatorname{Adm}(\eta)$.

Proof. We can work one component at a time and, thus, reduce to the case where $\# \mathcal{J}=1$. This can then be checked directly using Figure 2.

COROllary 2.3.13. Let $\lambda \in X_{1}(\underline{T})$ be 5-deep and $\mu-\eta \in \underline{C}_{0}$ be 3deep. If $F(\lambda) \notin \mathcal{R}\left(\mathrm{JH}\left(\overline{R_{s}(\mu)}\right)\right)$, then there exists a 3-generic Deligne-Lusztig representation $R_{s^{\prime}}\left(\mu^{\prime}\right)$ such that $F(\lambda) \in \mathrm{JH}\left(\overline{R_{s^{\prime}}\left(\mu^{\prime}\right)}\right)$ but

$$
\mathcal{R}\left(\mathrm{JH}\left(\overline{R_{s}(\mu)}\right)\right) \cap \mathrm{JH}\left(\overline{R_{s^{\prime}}\left(\mu^{\prime}\right)}\right)=\emptyset .
$$

Proof. If the central characters do not match, that is, $\left.(\lambda-\mu+\eta)\right|_{\underline{Z}} \notin(p-$ $\pi) X^{*}(\underline{Z})$, then any Deligne-Lusztig representation which contains $F(\lambda)$ works. Otherwise, by Proposition 2.1.4, there exists $(s \omega, a) \in \underline{\Lambda}_{W}^{\mu} \times \mathcal{A}$ such that 
Table 2. Intersections.

\begin{tabular}{|c|c|c|c|}
\hline$\widetilde{w} t_{-\underline{1}}$ & $\Sigma_{\widetilde{w}}$ & $\tilde{w} t_{-1}$ & $\Sigma_{\widetilde{w}}$ \\
\hline$\gamma^{+} \alpha \beta \alpha=t_{(1,0,-1)}$ & $\left\{\left(\varepsilon_{1}+\varepsilon_{2}, 0\right)\right\}$ & $\alpha \beta \alpha \gamma^{+}=t_{(-1,0,1)}$ & $\{(0,1)\}$ \\
\hline$\beta \gamma^{+} \beta \alpha=t_{(1,-1,0)}$ & $\left\{\left(\varepsilon_{1}, 1\right)\right\}$ & $\alpha \beta \gamma^{+} \beta=t_{(-1,1,0)}$ & $\left\{\left(\varepsilon_{2}-\varepsilon_{1}, 0\right)\right\}$ \\
\hline$\alpha \gamma^{+} \alpha \beta=t_{(0,1,-1)}$ & $\left\{\left(\varepsilon_{2}, 1\right)\right\}$ & $\beta \gamma^{+} \alpha \gamma^{+}=t_{(0,-1,1)}$ & $\left\{\left(\varepsilon_{1}-\varepsilon_{2}, 0\right)\right\}$ \\
\hline$\beta \alpha \gamma^{+}$ & $\left\{\left(\varepsilon_{1}-\varepsilon_{2}, 0\right),(\underline{0}, 1)\right\}$ & $\alpha \beta \gamma^{+}$ & $\left\{\left(\varepsilon_{2}-\varepsilon_{1}, 0\right),(0,1)\right\}$ \\
\hline$\alpha \beta \alpha$ & $\{(\underline{0}, 1),(\underline{0}, 0)\}$ & & \\
\hline$\beta \alpha$ & $\left\{\begin{array}{cc}(\underline{0}, 1), & (\underline{0}, 0) \\
\left(\varepsilon_{1}-\varepsilon_{2}, 0\right), & \left(\varepsilon_{1}, 1\right)\end{array}\right\}$ & $\alpha \beta$ & $\left\{\begin{array}{cc}(\underline{0}, 1), & (\underline{0}, 0) \\
\left(\varepsilon_{2}-\varepsilon_{1}, 0\right), & \left(\varepsilon_{2}, 1\right)\end{array}\right\}$ \\
\hline$\alpha$ & $\left\{\begin{array}{ccc}(\underline{0}, 1), & (\underline{0}, 0), & \left(\varepsilon_{2}, 1\right) \\
\left(\varepsilon_{2}, 0\right), & \left(\varepsilon_{2}-\varepsilon_{1}, 0\right), & \left(\varepsilon_{1}, 1\right)\end{array}\right\}$ & Id & $\left\{\begin{array}{lll}(\underline{0}, 1) & \left(\varepsilon_{1}, 1\right) & \left(\varepsilon_{2}, 1\right) \\
(\underline{0}, 0) & \left(\varepsilon_{1}, 0\right) & \left(\varepsilon_{2}, 0\right)\end{array}\right\}$ \\
\hline
\end{tabular}

Not all elements of $\operatorname{Adm}(\eta) t_{-1}$ appear in the table. There is an order-three symmetry of $\operatorname{Adm}(\eta) t_{-1}$ induced by the outer automorphism of $\widetilde{W}^{\text {der }}$, and we include at least one representative for each orbit.

$\sigma_{(\omega, a)}^{(s, \mu)} \cong F(\lambda)$. Moreover, since $F(\lambda) \notin \mathcal{R}\left(\mathrm{JH}\left(\overline{R_{s}(\mu)}\right)\right)$, we have $(\omega, a) \notin r(\Sigma)$. By Lemma 2.3.12, there exists $\widetilde{w} \in \underline{W}_{a} t_{\eta} \backslash \operatorname{Adm}(\eta)$ such that $(\omega, a) \in \widetilde{w}^{-1}(\Sigma)$. We take $s^{\prime}=s w^{-1}$ and $\mu^{\prime}=\mu+s \widetilde{w}^{-1}(0)$ so that $R_{s^{\prime}}\left(\mu^{\prime}\right)=R_{s \widetilde{w}^{-1}}(\mu)$. Since $(\omega$, a) $\in \widetilde{w}^{-1}(\Sigma), \omega-\widetilde{w}^{-1}(0)$ is in $\underline{W}(\bar{\Sigma})$, where $\bar{\Sigma}$ denotes the image of $\Sigma$ in $\underline{\Lambda}_{W}$. A direct computation shows that since $\lambda$ is 5-deep, $\mu+s \widetilde{w}^{-1}(0)-\eta$ is 3-deep in $\underline{C}_{0}$. By Proposition 2.3.7, we have that $F(\lambda) \in \mathrm{JH}\left(\overline{R_{s^{\prime}}\left(\mu^{\prime}\right)}\right)$. By Corollary 2.3.11 and the remark before it, we have that $\mathcal{R}\left(\mathrm{JH}\left(\overline{R_{s}(\mu)}\right)\right) \cap \mathrm{JH}\left(\overline{R_{s^{\prime}}\left(\mu^{\prime}\right)}\right)=\emptyset$.

\section{Serre weight conjectures}

In Sections 3.1-3.3, we improve results of [LLHLM18] on Kisin modules and étale $\varphi$-modules with descent data. Most of these results are generalized 


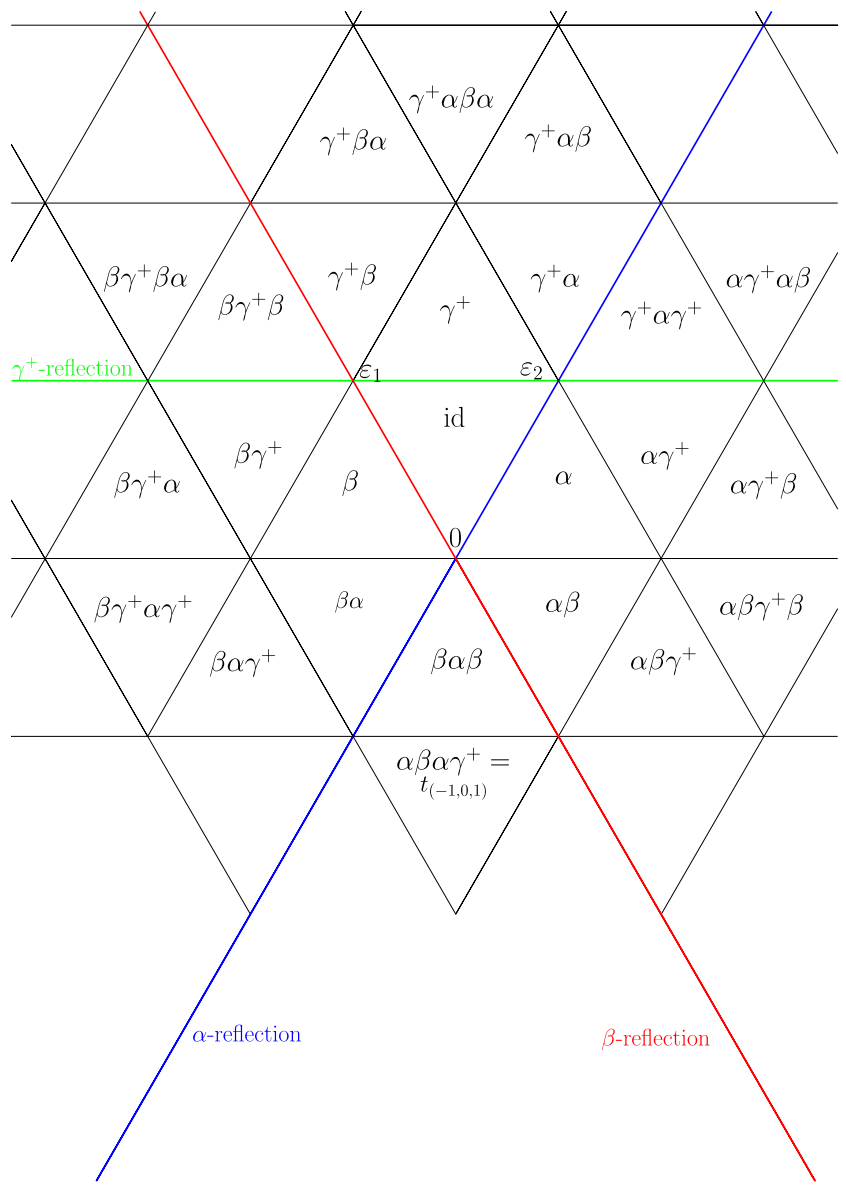

Figure 2. The standard apartment of $\mathrm{SL}_{3}$. Labeled alcoves are alcoves in $\operatorname{Adm}(\eta)$.

to $\mathrm{GL}_{n}$ in [LLHL19]. In Section 3.2, we prove a key structure result relating the Frobenius of a Kisin module with tame descent data to the Frobenius of the corresponding étale $\phi$-module (Proposition 3.2.1). This plays an important role in computing the Galois representation associated with semisimple Kisin modules (Proposition 3.3.8) and later establishing an explicit geometric BreuilMézard conjecture (see Section 3.6). The remainder of Section 3.3 is devoted to bounding reductions of potentially crystalline representations of type $(\eta, \tau)$ for the purpose of weight elimination (Theorem 3.3.12). 
In Section 3.4, we connect the results of Sections 3.1-3.3 with the combinatorics of Serre weights from Section 2.3. In particular, Proposition 3.4.2 describes the intersection of the set of predicted weights of a semisimple $\bar{\rho}$ with the set of Jordan-Hölder constituents of a Deligne-Lusztig representation, as a function of its shape. In Section 3.5, we prove the Serre weight conjectures for semisimple $\bar{\rho}$ by matching the number of predicted Serre weights to the number of irreducible components of the Galois deformation rings which were computed in [LLHLM18, Theorem 3.5.3]. In Section 3.6, we prove a geometric Breuil-Mézard conjecture which assigns to each (predicted) Serre weight a prime ideal of the universal framed deformation ring (Proposition 3.6.1). In Section 3.6.1, we make this assignment explicit for certain tamely potentially crystalline deformation rings. The explicit ideals play an important role in the proof of Breuil's lattice conjecture in Section 5.

Throughout this section, we assume that $\mathcal{S}=\{\widetilde{v}\}$ unless otherwise stated and write $K \stackrel{\text { def }}{=} F_{\widetilde{v}}$. We drop the subscript $\widetilde{v}$ from notation in this situation. The set $\mathcal{J}$ is identified with the set of embeddings $K \hookrightarrow E$ and also with $\mathbb{Z} / f \mathbb{Z}$ using our chosen embedding $\sigma_{0}$ (cf. Section 1.4). For $i \in \mathcal{J}$, we will use the word component $i$ and embedding $i$ interchangeably.

3.1. Background. In this subsection, we recall some basic facts on Kisin modules with tame descent data. We start with the following involution on $\underline{\widetilde{W}}$, which naturally appears when passing from Kisin modules to $G_{K_{\infty}}$ representations. We let $\widetilde{W}^{\vee}$ (respectively $\widetilde{W}^{\vee}$ ) be the partially ordered group which is identified with $\underline{\widetilde{W}}$ (respectively $\widetilde{W}$ ) as a group, and whose Bruhat order is defined by the antidominant base alcove (and still denoted as $\leqslant$ ).

DEFINITION 3.1.1. Define a bijection $\widetilde{w} \mapsto \widetilde{w}^{*}$ between $\widetilde{W}^{\vee}$ and $\underline{\widetilde{W}}$ as follows:

(1) For $w=\left(w_{j}\right) \in \underline{W}$, define $w^{*}=\left(w_{j}^{*}\right) \in \underline{W}$ by $w_{j}^{*}=w_{f-1-j}^{-1}$.

(2) For $v=\left(v_{j}\right) \in X^{*}(\underline{T})$, define $v^{*}=\left(v_{j}^{*}\right) \in X^{*}(\underline{T})$ by $v_{j}^{*}=v_{f-1-j}$.

(3) For $\widetilde{w}=w t_{v} \in \underline{\widetilde{W}}^{\vee}$, define $\widetilde{w}^{*} \in \underline{\widetilde{W}}$ by $\widetilde{w}^{*}=t_{v^{*}} w^{*}$.

Note that $\widetilde{w} \mapsto \widetilde{w}^{*}$ is an antihomomorphism of groups. For $j \in \mathcal{J}$, we write $\widetilde{w}_{j}^{*}$ for the $j$ th component of $\widetilde{w}^{*}$.

Let $\tau$ be a tame inertial type which we always assume to be 1-generic. Fix a lowest alcove presentation $(s, \mu)$ for $\tau$ (Definition 2.2.1).

If $s=\left(s_{0}, \ldots, s_{f-1}\right)$ and $\mu=\left(\mu_{j}\right)_{0 \leqslant j \leqslant f-1} \in X^{*}(\underline{T})$, we take

$$
s_{\tau} \stackrel{\text { def }}{=} s_{0} s_{f-1} s_{f-2} \cdots s_{1} \in W
$$


and $\boldsymbol{\alpha}_{(s, \mu)} \in X^{*}(\underline{T)})$ such that $\boldsymbol{\alpha}_{(s, \mu), j}=s_{1}^{-1} s_{2}^{-1} \ldots s_{j}^{-1}\left(\mu_{j}+\eta_{j}\right)$ for $1 \leqslant j \leqslant f-1$ and $\boldsymbol{\alpha}_{(s, \mu), 0}=\mu_{0}+\eta_{0}$. Let $r \in\{1,2,3\}$ be the order of $s_{\tau}$, and write $K^{\prime}$ for the unramified extension of $\mathbb{Q}_{p}$ of degree $f^{\prime} \stackrel{\text { def }}{=} f r$.

REMARK 3.1.2. In [LLHLM18], the notion of lowest alcove presentation does not appear. Everything is written for presentations of the form $\tau\left(\left(s_{\tau}, 1, \ldots, 1\right)\right.$, $\boldsymbol{\alpha}_{(s, \mu)}$ ) (see, for example, the beginning of [LLHLM18, Sections 2.1, 6.1]). In the notation of loc. cit., $\boldsymbol{\alpha}_{(s, \mu), j}=\left(a_{1, j}, a_{2, j}, a_{3, j}\right)$. The element

$$
s_{\text {or }} \stackrel{\text { def }}{=}\left(s_{1}^{-1} s_{2}^{-1} \ldots s_{f-1}^{-1}, s_{1}^{-1} s_{2}^{-1} \ldots s_{f-2}^{-1}, \ldots, s_{1}^{-1}, 1\right) \in \underline{W}
$$

has the property that $s_{\mathrm{or}}^{*}\left(\boldsymbol{\alpha}_{(s, \mu)}\right)=\mu+\eta$, and, hence, ${ }^{\left(0, s_{\mathrm{or}}^{*}\right)}\left(\left(\left(s_{\tau}, 1, \ldots, 1\right)\right.\right.$, $\left.\left.\boldsymbol{\alpha}_{(s, \mu)}\right)\right)=(s, \mu+\eta)$. The element $s_{\text {or }}$ is called the orientation of $\boldsymbol{\alpha}_{(s, \mu)}$ [LLHLM18, Definition 2.6 and equation (2.2)].

If $r=1$, we say that $\tau$ is a principal series type. Otherwise, we write $\tau^{\prime}$ for the base change of $\tau$ to $K^{\prime} / K$ (which is just $\tau$ considered as a principal series type for $\left.G_{K^{\prime}}\right)$. We record the relevant data for $\tau^{\prime}$. Define $\boldsymbol{\alpha}_{(s, \mu)}^{\prime} \in X^{*}(T)^{\operatorname{Hom}\left(k^{\prime}, \mathbb{F}\right)} \cong$ $X^{*}(\underline{T})^{r}$ (using a choice of embedding $\sigma_{0}^{\prime}: k^{\prime} \hookrightarrow \mathbb{F}$ extending $\sigma_{0}$ ) by

$$
\boldsymbol{\alpha}_{(s, \mu), j+k f}^{\prime} \stackrel{\text { def }}{=} s_{\tau}^{-k}\left(\boldsymbol{\alpha}_{(s, \mu), j}\right) \quad \text { for } 0 \leqslant j \leqslant f-1,0 \leqslant k \leqslant r-1 .
$$

If $\tau_{K^{\prime}}\left(w^{\prime}, \mu^{\prime}\right)$ is the analogous construction of tame types over $K^{\prime}$ for $\left(w^{\prime}\right.$, $\left.\mu^{\prime}\right) \in\left(\underline{W} \times X^{*}(\underline{T})\right)^{r}$ as in Definition 2.2.1, then $\tau^{\prime} \cong \tau_{K^{\prime}}\left(1, \boldsymbol{\alpha}_{(s, \mu)}^{\prime}\right)$ by direct comparison using equation (2.5). The orientation $s_{\text {or }}^{\prime} \in \underline{W}^{r}$ of $\boldsymbol{\alpha}_{(s, \mu)}^{\prime}$ in the sense of [LLHLM18, Definition 2.6] is given by

$$
s_{\mathrm{or}, j+k f}^{\prime} \stackrel{\text { def }}{=} s_{\tau}^{k+1} s_{\mathrm{or}, j} \quad \text { for } 0 \leqslant j \leqslant f-1,0 \leqslant k \leqslant r-1
$$

(compare with [LLHLM18, Proposition 6.1]).

Let $L^{\prime}=K^{\prime}\left(\pi_{r}\right)=K^{\prime}\left((-p)^{\frac{1}{p^{r f}-1}}\right)$ and $\Delta^{\prime} \stackrel{\text { def }}{=} \operatorname{Gal}\left(L^{\prime} / K^{\prime}\right) \subseteq \Delta \stackrel{\text { def }}{=} \operatorname{Gal}\left(L^{\prime} / K\right)$. Note that $\tau$ defines a $\mathcal{O}$-valued representation of $\Delta^{\prime}$. For any complete local Noetherian $\mathcal{O}$-algebra $R$ with residue field $\mathbb{F}^{\prime}$ finite over $\mathbb{F}$, let $\mathfrak{S}_{L^{\prime}, R} \stackrel{\text { def }}{=}$ $\left(W\left(k^{\prime}\right) \otimes_{\mathbb{Z}_{p}} R\right) \llbracket u^{\prime} \rrbracket$. We endow $\mathfrak{S}_{L^{\prime}, R}$ with an action of $\Delta$ as follows: for any $g$ in $\Delta^{\prime}, g\left(u^{\prime}\right)=\frac{g\left(\pi_{r}\right)}{\pi_{r}} u^{\prime}$ and $g$ acts trivially on the coefficients; if $\sigma \in \operatorname{Gal}\left(L^{\prime} / \mathbb{Q}_{p}\right)$ is the lift of Frobenius on $W\left(k^{\prime}\right)$ which fixes $\pi_{r}$, then $\sigma^{f}$ generates $\operatorname{Gal}\left(K^{\prime} / K\right)$ acting in natural way on $W\left(k^{\prime}\right)$ and trivially on both $u^{\prime}$ and $R$. Set $v=\left(u^{\prime}\right)^{p^{r f}-1}$, and note that

$$
\left(\mathfrak{S}_{L^{\prime}, R}\right)^{\Delta=1}=\left(W(k) \otimes_{\mathbb{Z}_{p}} R\right) \llbracket v \rrbracket .
$$

As usual, $\varphi: \mathfrak{S}_{L^{\prime}, R} \rightarrow \mathfrak{S}_{L^{\prime}, R}$ acts as $\sigma$ on $W\left(k^{\prime}\right)$, trivially on $R$, and sends $u^{\prime}$ to $\left(u^{\prime}\right)^{p}$. 
If $\tau$ is a principal series types, let $Y^{[0, h], \tau}(R)$ be the category of Kisin modules over $L^{\prime}$ with tame descent of type $\tau$ and height in $[0, h]$ as defined in [CL18, Section 3]. More generally, we have the following.

Definition 3.1.3. An element $\left(\mathfrak{M}, \phi_{\mathfrak{M}},\{\widehat{g}\}\right) \in Y^{[0, h], \tau}(R)$ is a Kisin module $\left(\mathfrak{M}, \phi_{\mathfrak{M}}\right)$ over $\mathfrak{S}_{L^{\prime}, R}$ [LLHLM18, Definition 2.3] with height less than $h$ together with a semilinear action of $\Delta$ which commutes with $\phi_{\mathfrak{M}}$ such that for each $0 \leqslant j \leqslant f^{\prime}-1$

$$
\mathfrak{M}^{(j)} \bmod u^{\prime} \cong \tau^{\vee} \otimes_{\mathcal{O}} R
$$

as $\Delta^{\prime}$-representations. In particular, the semilinear action induces an isomorphism $\iota_{\mathfrak{M}}:\left(\sigma^{f}\right)^{*}(\mathfrak{M}) \cong \mathfrak{M}$ (see [LLHLM18, Section 6.1]) as elements of $Y^{[0, h], \tau^{\prime}}(R)$.

REMARK 3.1.4. As explained in [LLHLM18, Section 6.1], the data of an extension of the action of $\Delta^{\prime}$ to an action of $\Delta$ is equivalent to the choice of an isomorphism $\iota_{\mathfrak{M}}:\left(\sigma^{f}\right)^{*}(\mathfrak{M}) \cong \mathfrak{M}$ satisfying an appropriate cocycle condition. We will use both points of view interchangeably.

REMARK 3.1.5. The appearance of $\tau^{\vee}$ in the definition is due to the fact that we are using the contravariant functors to Galois representations to be consistent with [LLHLM18] as opposed to the covariants versions which appear in [CL18, EGH13]. In [LLHLM18], we did not use the notation $\tau^{\vee}$. Instead, we included it in our description of descent data by having a minus sign in the equation before Definition 2.1 of loc. cit. The notion of Kisin module with tame descent data of type $\tau$ here is consistent with what appears in loc. cit.

Definition 3.1.6. An eigenbasis of $\mathfrak{M} \in Y^{[0, h], \tau}(R)$ is an eigenbasis $\beta^{\prime}=$ $\left(\beta^{\prime\left(j^{\prime}\right)}\right)_{j^{\prime}}$ for $\mathfrak{M}$ considered as a Kisin module with descent data of type $\tau^{\prime}$ in the sense of [LLHLM18, Definition 2.8], which is compatible with the isomorphism $\iota_{\mathfrak{M}}$ : by letting $\beta^{\prime\left(j^{\prime}\right)}=\left(f_{1}^{\prime\left(j^{\prime}\right)}, f_{2}^{\prime\left(j^{\prime}\right)}, f_{3}^{\prime\left(j^{\prime}\right)}\right)$ then

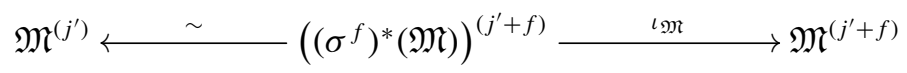

$$
\left\{f_{1}^{\prime\left(j^{\prime}\right)}, f_{2}^{\prime\left(j^{\prime}\right)}, f_{3}^{\prime\left(j^{\prime}\right)}\right\} \longmapsto\left\{f_{1}^{\prime\left(j^{\prime}+f\right)}, f_{2}^{\prime\left(j^{\prime}+f\right)}, f_{3}^{\prime\left(j^{\prime}+f\right)}\right\}
$$

for all $0 \leqslant j^{\prime} \leqslant f^{\prime}-1$, where the isomorphism on the left-hand side is obtained from [LLHLM18, Lemma 6.2]. For short, the compatibility above will be written as $\iota_{\mathfrak{M}}\left(\beta^{\left(j^{\prime}\right)}\right)=\beta^{\left(j^{\prime}+f\right)}$. (See also [LLHL19, Definition 3.2.8].) 
3.2. Étale $\varphi$-modules with descent data. The main result of this subsection is Proposition 3.2.1, describing the Frobenius action on étale $\varphi$-modules with tame descent data.

Let $\mathcal{O}_{\mathcal{E}, K^{\prime}}\left(\right.$ respectively $\left.\mathcal{O}_{\mathcal{E}, L^{\prime}}\right)$ be the $p$-adic completion of $\left(W\left(k^{\prime}\right) \llbracket v \rrbracket\right)[1 / v]$ (respectively of $\left.\left(W\left(k^{\prime}\right) \llbracket u^{\prime} \rrbracket\right)\left[1 / u^{\prime}\right]\right)$. Recall from [LLHLM18, Section 2.3] that for a complete local Noetherian $\mathcal{O}$-algebra $R$, we have the category $\Phi$ - $\operatorname{Mod}_{K^{\prime}}^{\text {ét }}(R)\left(\right.$ respectively $\Phi$ - $\left.\operatorname{Mod}_{\mathrm{dd}, L^{\prime}}^{\text {ét }}(R)\right)$ of étale $\left(\varphi, \mathcal{O}_{\mathcal{E}, K^{\prime}} \widehat{\otimes}_{\mathbb{Z}_{p}} R\right)$-modules (respectively étale $\left(\varphi, \mathcal{O}_{\mathcal{E}, L^{\prime}} \widehat{\bigotimes}_{\mathbb{Z}_{p}} R\right)$-modules with descent data from $L^{\prime}$ to $\left.K^{\prime}\right)$. There is an analogous definition of $\Phi$ - $\operatorname{Mod}_{K}^{\text {ét }}(R)$ and $\Phi-\operatorname{Mod}_{\mathrm{dd}, L}^{\text {ét }}(R)$. Given $(\mathfrak{M}$, $\left.\iota_{\mathfrak{M}}\right) \in Y^{[0, h], \tau}(R)$, the element $\mathfrak{M} \otimes \mathcal{O}_{\mathcal{E}, L^{\prime}}$ is naturally an object $\Phi$ - $\operatorname{Mod}_{\mathrm{dd}, L^{\prime}}^{\text {ét }}(R)$.

We define an étale $\varphi$-module $\mathcal{M} \in \Phi$ - $\operatorname{Mod}_{K}^{\text {ét }}(R)$ by

$$
\mathcal{M} \stackrel{\text { def }}{=}\left(\mathfrak{M} \otimes \mathcal{O}_{\mathcal{E}, L^{\prime}}\right)^{\Delta=1}
$$

with the induced Frobenius. This defines a functor from $Y^{[0, h], \tau}(R)$ to $\Phi-\operatorname{Mod}_{K}^{\text {ét }}(R)$. Finally, recall the functor $\varepsilon_{0}: \Phi-\operatorname{Mod}_{K}^{\text {ét }}(R) \rightarrow \Phi^{f}-\operatorname{Mod}_{W(k)}^{\text {ét }}(R)$ from [LLHLM18, Section 2.3]. It is obtained by considering the $f$-fold composite of the partial Frobenii which act on $\mathcal{M}^{(0)}$.

Let $R$ be a local, Artinian $\mathcal{O}$-algebra with finite residue field $\mathbb{F}$. We have the usual functor $\mathbb{V}_{K}^{*}$ from $\Phi$ - $\operatorname{Mod}_{K}^{\text {ét }}(R)$ to representations of $G_{K_{\infty}}$ over $R$. Recall from [LLHLM18, Section 6.1] the functor

$$
T_{\mathrm{dd}^{\prime}}^{*}: Y^{[0, h], \tau}(R) \rightarrow \operatorname{Rep}_{R}\left(G_{K_{\infty}}\right)
$$

which is defined as $\left(\mathfrak{M}, \iota_{\mathfrak{M}}\right) \mapsto \mathbb{V}_{K}^{*}(\mathcal{M})$. From now onward, we write $T_{\mathrm{dd}}^{*}$ for the functor which was written as $T_{\mathrm{dd}^{\prime}}^{*}$ in [LLHLM18, Section 6.1].

We can now state the main result of this section, which is a sharpening of [LLHLM18, Proposition 2.26].

PROPOSITION 3.2.1. Let $\tau$ be a 1-generic type with lowest alcove presentation $(s, \mu)$. Let $\mathfrak{M} \in Y^{[0, h], \tau}(R)$ and $\beta$ be an eigenbasis of $\mathfrak{M}$. Let $s_{\mathrm{or}}=\left(s_{\mathrm{or}, j}\right)_{j}$ be the orientation for $\tau$ and write $A^{(j)}=\operatorname{Mat}_{\beta}\left(\phi_{\mathfrak{M}, s_{\mathrm{or}, j+1}(3)}^{(j)}\right)$ for $j \in\{0, \ldots, f-1\}$. Let $\mathcal{M}=\left(\mathfrak{M} \otimes \mathcal{O}_{\mathcal{E}, L^{\prime}}\right)^{\Delta=1} \in \Phi-\operatorname{Mod}_{K}^{e t}(R)$.

Then there is a basis $\mathfrak{f}=\left(\mathfrak{f}^{(j)}\right)_{j}$ for $\mathcal{M}$ such that

$$
\operatorname{Mat}_{\mathfrak{f}}\left(\phi_{\mathcal{M}}^{(j)}\right)=A^{(j)} s_{j}^{*} v^{\mu_{j}^{*}+\eta_{j}^{*}}
$$

Proof. The proof is a direct computation using [LLHLM18, Propositions 2.26 and 6.1]. 
Let $\boldsymbol{\alpha}_{(s, \mu)}^{\prime}$ and $\left(s_{\mathrm{or}, j^{\prime}}^{\prime}\right)$ be as in the discussion after Remark 3.1.2. Let $\beta^{\left(j^{\prime}\right)}=$ $\left(f_{1}^{\prime\left(j^{\prime}\right)}, f_{2}^{\prime\left(j^{\prime}\right)}, f_{3}^{\prime\left(j^{\prime}\right)}\right)$ be an eigenbasis for $\mathfrak{M}$ and write $A^{\left(j^{\prime}\right)} \stackrel{\text { def }}{=} \operatorname{Mat}_{\beta^{\prime}}\left(\phi_{\mathfrak{M}^{\prime}, s_{\text {or }, j^{\prime}+1}\left(j^{\prime}\right)}\right)$ for $0 \leqslant j^{\prime}<f^{\prime}-1$.

For any $0 \leqslant j^{\prime} \leqslant f^{\prime}-1$, set

$$
\mathbf{a}_{(s, \mu)}^{\prime\left(j^{\prime}\right)}=\sum_{i=0}^{f^{\prime}-1} \boldsymbol{\alpha}_{(s, \mu),-j^{\prime}+i}^{\prime} p^{i} \in \mathbb{Z}^{3},
$$

where $-j^{\prime}+i$ is taken modulo $f^{\prime}$ (compare with [LLHLM18, Section 2.1]). Then

$$
\mathfrak{f}^{\prime\left(j^{\prime}\right) \stackrel{\operatorname{def}}{=}}\left\{\left(u^{\prime}\right)^{\mathbf{a}_{(s, \mu), 1}^{\left(j^{\prime}\right)}} f_{1}^{\prime\left(j^{\prime}\right)},\left(u^{\prime}\right)^{\mathbf{a}_{(s, \mu), 2}^{\left(j^{\prime}\right)}} f_{2}^{\prime\left(j^{\prime}\right)},\left(u^{\prime}\right)^{\mathbf{a}_{(s, \mu), 3}^{\left(j^{\prime}\right)}} f_{3}^{\prime\left(j^{\prime}\right)}\right\}
$$

is a basis for $\mathcal{M}^{\prime\left(j^{\prime}\right)} \stackrel{\text { def }}{=}\left(\mathfrak{M}^{\left(j^{\prime}\right)}\left[1 / u^{\prime}\right]\right)^{\Delta^{\prime}=1}$ for $0 \leqslant j^{\prime}<f^{\prime}-1$.

For any $0 \leqslant j^{\prime} \leqslant f^{\prime}-1$, a direct computation (using [LLHLM18, Proposition 2.13]) shows that the matrix for $\phi_{\mathcal{M}^{\prime}}^{\left(j^{\prime}\right)}: \mathcal{M}^{\prime\left(j^{\prime}\right)} \rightarrow \mathcal{M}^{\left(j^{\prime}+1\right)}$ with respect to the bases above is

$$
s_{\mathrm{or}, j^{\prime}+1}^{\prime} A^{\left(j^{\prime}\right)}\left(s_{\mathrm{or}, j^{\prime}+1}^{\prime}\right)^{-1}\left(u^{\prime}\right)^{p \mathbf{a}_{(s, \mu)}^{\left(j^{\prime}\right)}-\mathbf{a}_{(s, \mu)}^{\prime\left(j^{\prime}+1\right)}} .
$$

Since $p \mathbf{a}_{(s, \mu)}^{\left(j^{\prime}\right)}-\mathbf{a}_{(s, \mu)}^{\prime\left(j^{\prime}+1\right)}=\left(p^{f^{\prime}}-1\right) \boldsymbol{\alpha}_{(s, \mu), f^{\prime}-1-j^{\prime}}^{\prime}$, this is same as

$$
s_{\mathrm{or}, j^{\prime}+1}^{\prime} A^{\left(j^{\prime}\right)}\left(s_{\mathrm{or}, j^{\prime}+1}^{\prime}\right)^{-1} v^{\alpha_{(s, \mu), f^{\prime}-1-j^{\prime}}^{\prime}} .
$$

Define $\widetilde{\beta}$ by $\widetilde{\beta}^{\left(j^{\prime}\right)}=\mathfrak{f}^{\left(j^{\prime}\right)} s_{\text {or }, j^{\prime}}^{\prime}$ (reordering the basis vectors). Let $j^{\prime}=j+i f$ for $0 \leqslant j \leqslant f-1$. Then the matrix for $\phi_{\mathcal{M}^{\prime}}^{\left(j^{\prime}\right)}$ with respect to $\widetilde{\beta}$ is given by

$$
A^{\left(j^{\prime}\right)}\left(s_{\mathrm{or}, j^{\prime}+1}^{\prime}\right)^{-1} s_{\mathrm{or}, j^{\prime}}^{\prime} v^{\left(s_{\mathrm{or}, j^{\prime}}^{\prime}\right)^{-1}\left(\alpha_{(s, \mu), f^{\prime}-1-j^{\prime}}^{\prime}\right)}=A^{(j)} s_{j}^{*} v^{\mu_{j}^{*}+\eta_{j}^{*}}
$$

using equation (3.1), Remark 3.1.2, and the fact that $A^{\left(j^{\prime}\right)}$ only depends on $j^{\prime}$ $\bmod f$ (cf. [LLHLM18, Proposition 6.9]).

Finally, recall that the eigenbasis $\beta^{\prime}$ is required to be compatible with $\iota_{\mathfrak{M}}$ : $\left(\sigma^{f}\right)^{*}(\mathfrak{M}) \cong \mathfrak{M}$ as in Definition 3.1.6, which gives $\iota_{\mathfrak{M}}^{\left(j^{\prime}\right)}\left(\beta^{\prime\left(j^{\prime}\right)}\right)=\beta^{\prime\left(j^{\prime}+f\right)} s_{\tau}^{-1}$. Hence, $\widetilde{\beta}$ descends to a collection of ordered bases $\left(\mathfrak{f}^{(j)}\right)_{0 \leqslant j \leqslant f-1}$ of the $\mathcal{M}^{(j)}=$ $\left(\left(\mathfrak{M}\left[1 / u^{\prime}\right]\right)^{\Delta=1}\right)^{(j)}$ such that the matrix for the partial Frobenius map $\mathcal{M}^{(j)} \rightarrow$ $\mathcal{M}^{(j+1)}$ is given by equation (3.3).

Proposition 3.2.2. Let $\mathfrak{M} \in Y^{[0, h], \tau}(R)$ and let $\mathcal{M}=\left(\mathfrak{M} \otimes \mathcal{O}_{\mathcal{E}, L^{\prime}}\right)^{\Delta=1} \in$ $\Phi-\operatorname{Mod}_{K}^{e t}(R)$ be the associated étale $\varphi$-module over $K$. 
If $\mathfrak{f}$ is a basis of $\mathcal{M}$ and $B^{(j)} \stackrel{\text { def }}{=} \operatorname{Mat}_{\mathfrak{f}}\left(\phi_{\mathcal{M}}^{(j)}\right)$ are the matrices of the partial Frobenii, then the étale $\varphi^{f}$-module $\varepsilon_{0}(\mathcal{M})$ is described by

$$
\operatorname{Mat}_{\mathfrak{f}^{(0)}}\left(\phi_{\mathcal{M}^{(0)}}^{f}\right)=\prod_{j=0}^{f-1} \varphi^{j}\left(B^{(f-j-1)}\right) .
$$

LEMMA 3.2.3. Let $\bar{\rho}: G_{K} \rightarrow \mathrm{GL}_{3}(\mathbb{F})$ be semisimple and 0 -generic. Let $\mathcal{M} \in$ $\Phi^{f}-\operatorname{Mod}_{W(k)}^{e t}(\mathbb{F})$ such that $\left.\mathbb{V}_{K}^{*}(\mathcal{M}) \cong \bar{\rho}\right|_{G_{K \infty}}$. Assume that there exists $s_{0} \in W$, $\lambda=\left(\lambda_{j}\right) \in X_{1}^{*}(\underline{T})$, and a basis for $\mathcal{M}$ such that the $\phi^{f}$-action on $\mathcal{M}$ is given by $D s_{0}^{-1} v^{\mu}$ where $D \in T(\mathbb{F})$ and $\mu \stackrel{\text { def }}{=} \sum_{j=0}^{f-1} p^{j} \lambda_{j} \in X^{*}(T)$. Then by letting $s=\left(s_{0}, 1, \ldots, 1\right) \in \underline{W}$,

$$
V\left(\left.\bar{\rho}\right|_{I_{K}}\right)=R_{s}(\lambda)
$$

Proof. Let $k$ be the order of $s_{0}$ and write $\mu=\left(\mu_{1}, \mu_{2}, \mu_{3}\right) \in X^{*}(T)$. Then

$$
\begin{aligned}
\phi^{f k}\left(e_{i}\right) & =\left(\prod_{m=0}^{k-1} D_{s_{0}^{-m}(i)}\right) v^{\sum_{m=0}^{k-1} p^{f(k-1-m)} \mu_{s_{0}^{-m}(i)}} e_{i} \\
& =\left(\prod_{m=0}^{k-1} D_{s_{0}^{m+1}(i)}\right) v^{\sum_{m=0}^{k-1} p^{f m} \mu_{s_{0}^{m+1}(i)}} e_{i} .
\end{aligned}
$$

Hence, $\left.\bar{\rho}\right|_{I_{K}}$ is isomorphic to $\bigoplus_{i=1}^{3} \omega_{k f}^{\sum_{m=0}^{k-1} p^{f m} \mu_{s_{0}^{m+1}(i)}}$. The lemma now follows from (2.5).

3.3. Semisimple Kisin modules. The statement of [LLHLM18, Theorem 2.21] gives a classification of Kisin modules with descent datum which can arise as reductions of potentially crystalline representations with Hodge-Tate weights $(2,1,0)^{f}$. In this subsection, we identify Kisin modules which correspond to semisimple Galois representations in an explicit way (Theorem 3.3.12).

For $\mathbb{F}^{\prime} / \mathbb{F}$ finite, let $\mathcal{I}\left(\mathbb{F}^{\prime}\right) \subset \mathrm{GL}_{3}\left(\mathbb{F}^{\prime} \llbracket v \rrbracket\right)$ be the Iwahori subgroup of elements which are upper triangular modulo $v$. Recall from Section 3.1 the partially ordered group $\widetilde{W}^{\vee}$ (respectively $\widetilde{W}^{\vee}$ ) which is identified with $\underline{\widetilde{W}}$ (respectively $\widetilde{W}$ ) as a group but whose Bruhat order is defined by the antidominant base alcove (and still denoted as $\leqslant$ ). For any character $\mu \in X^{*}(\underline{T})$, we define $\operatorname{Adm}^{\vee}(\mu)$ as in Definition 2.3.9 but for antidominant base alcove.

Let $h$ be a positive integer. As before, let $\tau$ be a 1-generic tame type with fixed lowest alcove presentation $(s, \mu)$. Recall from [LLHLM18, Definition 2.17] the notion of shape. 
DEFINITION 3.3.1. Let $\tau$ be a principal series type, and let $\widetilde{w}=\left(\widetilde{w}_{0}, \widetilde{w}_{1}, \ldots\right.$, $\left.\widetilde{w}_{f-1}\right) \in \underline{\widetilde{W}}^{\vee}$. A Kisin module $\mathfrak{M} \in Y^{[0, h], \tau}\left(\mathbb{F}^{\prime}\right)$ has shape $\widetilde{w}$ if for any eigenbasis $\beta$, the matrices $\left(A^{(j)}\right)_{j}=\left(\operatorname{Mat}_{\beta}\left(\phi_{\mathfrak{M}, s_{\mathrm{or}, j+1}(3)}^{(j)}\right)\right)_{j}$ have the property that $A^{(j)} \in$ $\mathcal{I}\left(\mathbb{F}^{\prime}\right) \widetilde{w}_{j} \mathcal{I}\left(\mathbb{F}^{\prime}\right)$.

For a nonprincipal series type, we define the shape of a Kisin module $\mathfrak{M} \in$ $Y^{[0, h], \tau}\left(\mathbb{F}^{\prime}\right)$ in terms of its base change BC $(\mathfrak{M})$; see [LLHL19, Definition 3.2.11] or [LLHLM18, Definition 6.10] for details.

For any $\lambda \in X^{*}(\underline{T})$ effective (that is, $\lambda_{j}=\left(a_{i, j}\right)_{i}$ with $a_{i, j} \geqslant 0$ for all $i, j$ ) and $h \gg 0$, let $Y^{\lambda, \tau} \subset Y^{[0, h], \tau}$ denote the closed substack defined in [CL18, Section 5] (cf. also [LLHLM18, Section 2.2]). Then for any finite extension $\mathbb{F}^{\prime} / \mathbb{F}, Y^{\lambda, \tau}\left(\mathbb{F}^{\prime}\right) \subset Y^{[0, h], \tau}\left(\mathbb{F}^{\prime}\right)$ is the subgroupoid consisting of Kisin modules with shape in $\operatorname{Adm}^{\vee}(\lambda)$.

DEFINITION 3.3.2. Let $\tau$ be a 3-generic type with lowest alcove presentation $(s, \mu)$ and let $\bar{\rho}: G_{K} \rightarrow \mathrm{GL}_{3}(\mathbb{F})$. Assume that there exists $\overline{\mathfrak{M}}_{\bar{\rho}} \in Y^{\eta, \tau}(\mathbb{F})$ such that $\left.T_{\mathrm{dd}}^{*}\left(\overline{\mathfrak{M}}_{\bar{\rho}}\right) \cong \bar{\rho}\right|_{G_{K_{\infty}}}$. We define $\widetilde{w}(\bar{\rho}, \tau) \in \operatorname{Adm}^{\vee}(\eta)$ to be the shape of $\overline{\mathfrak{M}}_{\bar{\rho}}$.

This is well defined because there exists at most one Kisin module $\overline{\mathfrak{M}}_{\bar{\rho}}$ with height in [0, 2] such that $\left.T_{\mathrm{dd}}^{*}\left(\overline{\mathfrak{M}}_{\bar{\rho}}\right) \cong \bar{\rho}\right|_{G_{K \infty}}$ (see [LLHLM18, Theorem 3.2] for a principal series type and Section 6.2 and the discussion before Lemma 6.13 in loc. cit. for the general case).

REMARK 3.3.3. More generally, if $\# \mathcal{S}>1$, for $\widetilde{w}=\left(\widetilde{w}_{\widetilde{v}}\right)_{\widetilde{v} \in \mathcal{S}}$, we define $\widetilde{w}^{*}$ as the collection $\left(\widetilde{w}_{\widetilde{v}}^{*}\right)_{\widetilde{v} \in \mathcal{S}}$, where each $\widetilde{w}_{\widetilde{v}}^{*} \in \widetilde{W}_{\widetilde{v}}$ is as in Definition 3.1.1. Similarly, if $\tau_{\mathcal{S}}$ is a collection of 3-generic tame inertial type and $\bar{\rho}_{\mathcal{S}}$ is a collection of Galois representations, we let $\widetilde{w}\left(\bar{\rho}_{\mathcal{S}}, \tau_{\mathcal{S}}\right) \stackrel{\text { def }}{=}\left(\widetilde{w}\left(\bar{\rho}_{\widetilde{v}}, \tau_{\widetilde{v}}\right)\right)_{\widetilde{v} \in \mathcal{S}}$.

We now introduce the notion of semisimple Kisin module of shape $\widetilde{w}$.

Definition 3.3.4. Let $\overline{\mathfrak{M}} \in Y^{[0, h], \tau}\left(\mathbb{F}^{\prime}\right)$, where $\mathbb{F}^{\prime} / \mathbb{F}$ is a finite extension. We say that $\overline{\mathfrak{M}}$ is semisimple of shape $\widetilde{w}=\left(\widetilde{w}_{j}\right)$ if there exists an eigenbasis $\beta$ of $\overline{\mathfrak{M}}$ such that

$$
A^{(j)} \in T\left(\mathbb{F}^{\prime} \llbracket v \rrbracket\right) \widetilde{w}_{j}
$$

for $0 \leqslant j \leqslant f^{\prime}-1$.

Proposition 3.3.5. If $\overline{\mathfrak{M}}$ is semisimple of shape $\widetilde{w}$, then $T_{\mathrm{dd}}^{*}(\overline{\mathfrak{M}})$ is semisimple.

Proof. If $\overline{\mathfrak{M}}$ is semisimple, we can choose a basis such that $A^{(j)} \in T\left(\mathbb{F}^{\prime} \llbracket v \rrbracket\right) \widetilde{w}_{j}$ where $\mathbb{F}^{\prime} / \mathbb{F}$ is a finite extension. Using this basis, we see by Propositions 3.2.1 
and 3.2.2 that the matrix for the étale $\varphi^{f}$-module $\varepsilon_{0}\left(\overline{\mathfrak{M}}\left[1 / u^{\prime}\right]^{\Delta=1}\right)$ lies in $T\left(\mathbb{F}^{\prime}((v))\right) s$ for some $s \in W$. If $s$ has order $d$, then $T_{\mathrm{dd}}^{*}(\overline{\mathfrak{M}})$ restricted to the unramified extension of $K_{\infty}$ of degree $d$ is a direct sum of characters (as $p \nmid d$ ) and so $T_{\mathrm{dd}}^{*}(\overline{\mathfrak{M}})$ is semisimple.

PROPOSITION 3.3.6. If $\overline{\mathfrak{M}}$ is semisimple of shape $\widetilde{w}=\left(\widetilde{w}_{j}\right)$, then there exists an eigenbasis $\beta$ such that

$$
A^{(j)}=\widetilde{w}_{j} \quad \text { for } 0 \leqslant j \leqslant f-2, \quad A^{(f-1)} \in T\left(\mathbb{F}^{\prime}\right) \widetilde{w}_{f-1} .
$$

Proof. See [LLHL19, Proposition 3.2.16].

Definition 3.3.7. For any $\widetilde{w}=\left(w_{j} t_{\lambda_{j}}\right) \in \widetilde{W}^{\vee}$, define $\mathcal{M}(\widetilde{w}) \in \Phi-\operatorname{Mod}_{K}^{\text {ét }}(\mathbb{F})$ to be the free étale $\varphi$-module over $\mathcal{O}_{\mathcal{E}, K} \otimes \mathbb{F}$ of rank three such that the matrix of

$$
\phi_{\mathcal{M}}^{(j)}: \mathcal{M}(\widetilde{w})^{(j)} \rightarrow \mathcal{M}(\widetilde{w})^{(j+1)}
$$

is given by $w_{j} v^{\lambda_{j}}$ (with respect to the standard basis).

Proposition 3.3.8. Let $(s, \mu)$ be a lowest alcove presentation of $\tau$. If $\overline{\mathfrak{M}} \in$ $Y^{[0, h], \tau}\left(\mathbb{F}^{\prime}\right)$ is semisimple of shape $\widetilde{w} \in \underline{\widetilde{W}}^{\vee}$, then

$$
\left.\left.T_{\mathrm{dd}}^{*}(\overline{\mathfrak{M}})\right|_{I_{K}} \cong \mathbb{V}_{K}^{*}\left(\mathcal{M}\left(\widetilde{w} s^{*} t_{\mu^{*}+\eta^{*}}\right)\right)\right|_{I_{K}} \cong \bar{\tau}(w, v+\eta)
$$

where $\widetilde{w} s^{*} t_{\mu^{*}}=w^{*} t_{v^{*}}$. (Note that since both $T_{\mathrm{dd}}^{*}(\overline{\mathfrak{M}})$ and $\mathbb{V}_{K}^{*}\left(\mathcal{M}\left(\widetilde{w} s^{*} t_{\mu^{*}+\eta^{*}}\right)\right)$ are tame, they canonically extend to $G_{K}$.)

Proof. This is the special case when $n=3$ of [LLHL19, Corollary 3.2.17]. For the sake of completeness, we include an argument here.

The first isomorphism follows from Proposition 3.3.6 combined with Proposition 3.2.1.

Let $w, v$ satisfy $\widetilde{w} s^{*} t_{\mu^{*}}=w^{*} t_{v^{*}}$. One easily checks that the $\phi^{f}$-action on $\varepsilon_{0}\left(\mathcal{M}\left(w^{*} t_{v^{*}+\eta^{*}}\right)\right) \in \Phi^{f}-\operatorname{Mod}_{W(k)}^{\text {ét }}(\mathbb{F})$ is given by $\prod_{j=0}^{f-1} w_{j}^{-1} v^{p^{j}\left(v_{j}+\eta_{j}\right)}$ using Proposition 3.2.2.

Let $s \in \underline{W}$. The $\phi^{f}$-action of $\varepsilon_{0}\left(\mathcal{M}\left(\left(s t_{v+\eta} w \pi(s)^{-1}\right)^{*}\right)\right) \in \Phi^{f}-\operatorname{Mod}_{W(k)}^{\text {ét }}(\mathbb{F})$ is given by

$$
\prod_{j=0}^{f-1} s_{j-1} w_{j}^{-1} s_{j}^{-1} v^{p^{j} s_{j}\left(v_{j}+\eta_{j}\right)}=s_{f-1}\left(\prod_{j=0}^{f-1} w_{j}^{-1} v^{p^{j}\left(v_{j}+\eta_{j}\right)}\right) s_{f-1}^{-1} .
$$

We conclude that $\varepsilon_{0}\left(\mathcal{M}\left(w^{*} t_{v^{*}+\eta^{*}}\right)\right) \cong \varepsilon_{0}\left(\mathcal{M}\left(\left(s t_{v+\eta} w \pi(s)^{-1}\right)^{*}\right)\right)$. Therefore, $\mathbb{V}_{K}^{*}\left(\mathcal{M}\left(w^{*} t_{v^{*}+\eta^{*}}\right)\right) \cong \mathbb{V}_{K}^{*}\left(\mathcal{M}\left(\left(s t_{v+\eta} w \pi(s)^{-1}\right)^{*}\right)\right)$. 
As $(w, v+\eta) \sim\left(s w \pi(s)^{-1}, s(v+\eta)\right)$, by [LLHL19, Proposition 2.2.4] and [Her09, Lemma 4.2], we reduce to the case where $w_{i}=1$ for $i \neq f-1$. The second isomorphism then follows from Lemma 3.2.3.

Proposition 3.3.9. Let $\bar{\rho}, \bar{\rho}^{\prime}$ be semisimple representations of $G_{K}$. Assume that there exists $\overline{\mathfrak{M}}$ semisimple of shape $\widetilde{w}$ such that $\left.T_{\mathrm{dd}}^{*}(\overline{\mathfrak{M}}) \cong \bar{\rho}\right|_{G_{K_{\infty}}}$. Then $\left.\left.\bar{\rho}^{\prime}\right|_{I_{K}} \cong \bar{\rho}\right|_{I_{K}}$ if and only if there exists a semisimple $\overline{\mathfrak{M}}^{\prime}$ of shape $\widetilde{w}$ such that $\left.T_{\mathrm{dd}}^{*}\left(\overline{\mathfrak{M}^{\prime}}\right) \cong \bar{\rho}\right|_{G_{K_{\infty}}}$.

Proof. This follows from counting unramified twists using Proposition 3.3.6.

The remainder of the subsection is devoted to results used for weight elimination.

LEMMA 3.3.10. If $\bar{\rho}: G_{K} \rightarrow \mathrm{GL}_{3}(\mathbb{F})$ admits a potentially crystalline lift of type $(\lambda, \tau)$, then so does $\bar{\rho}^{\mathrm{ss}}$ (after possibly replacing $E$ with a ramified extension).

Proof. Fix a characteristic 0 lift $\rho$ of $\bar{\rho}$ which is potentially crystalline of type $(\lambda, \tau)$. Then by enlarging the coefficient ring of $\rho$, we can always find a lattice whose reduction is semisimple by [Enn, Lemma 5(2)].

The following two theorems are key inputs in weight elimination by giving an upper bound on the semisimple representations which are reductions of potentially crystalline representations of type $(\lambda, \tau)$.

THEOREM 3.3.11. Let $\bar{\rho}: G_{K} \rightarrow \mathrm{GL}_{3}(\mathbb{F})$. Assume that $\bar{\rho}$ has a potentially crystalline lift of type $(\lambda, \tau)$ where $\lambda \in X^{*}(\underline{T})$ is effective. Assume that either (1) $\tau$ is a regular principal series type or (2) $\lambda=\eta$ and $\tau$ is 3-generic. Then, for a sufficiently large $h$, there is a Kisin module $\overline{\mathfrak{M}} \in Y^{[0, h], \tau}(\mathbb{F})$ of shape $\widetilde{w}=$ $\left(\widetilde{w}_{0}, \widetilde{w}_{1}, \ldots, \widetilde{w}_{f-1}\right) \in \operatorname{Adm}^{\vee}(\lambda)$ such that $\left.T_{\mathrm{dd}}^{*}(\overline{\mathfrak{M}}) \cong \bar{\rho}\right|_{G_{K_{\infty}}}$. In particular, $\overline{\mathfrak{M}} \in$ $Y^{\lambda, \tau}(\mathbb{F})$.

Proof. See [LLHL19, Theorem 3.2.20].

THEOREM 3.3.12. Let $\bar{\rho}$ be a semisimple representation of $G_{K}$ and assume $\tau$ is a 1-generic tame type. Assume that either (1) $\bar{\rho}$ is a direct sum of characters or (2) $\lambda=\eta$ and $\tau$ is 3-generic.

If there exists a Kisin module $\overline{\mathfrak{M}} \in Y^{\lambda, \tau}(\mathbb{F})$ such that $\left.T_{\mathrm{dd}}^{*}(\overline{\mathfrak{M}}) \cong \bar{\rho}\right|_{G_{K_{\infty}}}$, then there exists a finite extension $\mathbb{F}^{\prime} / \mathbb{F}$ and a semisimple Kisin module $\overline{\mathfrak{M}}^{\prime} \in Y^{\lambda, \tau}\left(\mathbb{F}^{\prime}\right)$ 
such that (after extending scalars) $\left.T_{\mathrm{dd}}^{*}\left(\overline{\mathfrak{M}}^{\prime}\right) \cong \bar{\rho}\right|_{G_{K_{\infty}}}$. Furthermore, we can take $\mathbb{F}^{\prime}=\mathbb{F}$ in case $(2)$.

REMARK 3.3.13. Theorems 3.3.11 and 3.3.12 together say that for a fixed 3generic type $\tau$ the set of semisimple $\left.\bar{\rho}\right|_{I_{K}}$ which arise as reductions of potentially crystalline representations of type $(\eta, \tau)$ are in bijection with a subset of $\operatorname{Adm}^{\vee}((2,1,0))^{f}$. In fact, it turns out that this admissibility condition exactly captures those semisimple $\bar{\rho}$ which are reductions of crystalline representations of type $(\eta, \tau)$. Checking this is equivalent to checking that the potentially crystalline deformation ring of type $(\eta, \tau)$ of the semisimple $\bar{\rho}$ corresponding to $\widetilde{w} \in \operatorname{Adm}^{\vee}((2,1,0))^{f}$ is nonzero. When $\widetilde{w}$ is such that $\ell\left(\widetilde{w}_{i}\right)>1$ for all $i$, this nontriviality follows from [LLHLM18, Table 7]. When $K=\mathbb{Q}_{p}$, [LLHLM18, Section 8] shows the nontriviality for all admissible $\widetilde{w}$. For general unramified $K$ and admissible $\widetilde{w}$, the nontriviality of the deformation ring will follow from Theorem 3.5.3.

Proof of Theorem 3.3.12. We first treat the case where $\bar{\rho}$ is a direct sum of characters. Let $\mathcal{M}_{\mathrm{dd}}=\overline{\mathfrak{M}}\left[1 / u^{\prime}\right]$ be an étale $\varphi$-module with descent datum for $L^{\prime} / K$ (that is, a semilinear action of $\Delta$ ). Since there is an equivalence of categories between étale $\varphi$-modules over $L^{\prime}$ with descent datum to $K$ and $G_{K_{\infty}}$-representations (cf. [LLHLM18, page 24] for principal series case and Section 6.1 in general), if $\bar{\rho}$ is a direct sum of characters, then $\mathcal{M}_{\mathrm{dd}}=\mathcal{M}_{1} \oplus$ $\mathcal{M}_{2} \oplus \mathcal{M}_{3}$, where each $\mathcal{M}_{i}$ is stable under $\phi_{\mathcal{M}_{\mathrm{dd}}}$ and the descent datum.

Let $Y_{\mathcal{M}_{\mathrm{dd}}, \tau}$ be the Kisin variety parametrizing lattices in $\mathcal{M}_{\mathrm{dd}}$ which lie in $Y^{\lambda, \tau}$. [LLHLM18, Definition 3.1] defines $Y_{\mathcal{M}_{\mathrm{dd}}}^{\lambda, \tau}$ in the principal series case. In general, $Y_{\mathcal{M}_{\mathrm{dd}}}^{\lambda, \tau}$ is the closed subscheme of fixed points of $Y_{\mathcal{M}_{\mathrm{dd}}}^{\lambda, \tau^{\prime}}$ under the natural action of $\sigma^{f}$. The torus $T=\mathbb{G}_{m}^{3}$ acts on $\mathcal{M}_{\mathrm{dd}}$ by scaling individually in each factor. As a consequence, we get an algebraic action of $T$ on the projective variety $Y_{\mathcal{M}_{\mathrm{dd}}}^{\lambda, \tau}$.

Any such action has a fixed point over some finite extension $\mathbb{F}^{\prime} / \mathbb{F}$. Let $\overline{\mathfrak{M}}^{\prime} \subset$ $Y_{\mathcal{M}_{\mathrm{dd}}}^{\lambda, \tau}\left(\mathbb{F}^{\prime}\right)$ be a $T$-fixed point. Let $\chi_{i}: T \rightarrow \mathbb{G}_{m}$ denote the projection onto each coordinate and set $\overline{\mathfrak{M}}_{i}^{\prime} \stackrel{\text { def }}{=}\left(\overline{\mathfrak{M}}^{\prime}\right)^{\chi_{i}}$. Then

$$
\overline{\mathfrak{M}}^{\prime}=\overline{\mathfrak{M}}_{1}^{\prime} \oplus \overline{\mathfrak{M}}_{2}^{\prime} \oplus \overline{\mathfrak{M}}_{3}^{\prime} .
$$

Since the $T$-action commutes with $\phi_{\mathcal{M}_{\mathrm{dd}}}$ and $\Delta$, each $\overline{\mathfrak{M}}_{i}^{\prime}$ is stable under both; hence, $\overline{\mathfrak{M}}_{i}^{\prime}$ is a rank-one Kisin module with descent datum. Any choice of eigenbasis which respects this decomposition shows that $\overline{\mathfrak{M}}^{\prime}$ is semisimple. Because $\overline{\mathfrak{M}}^{\prime}$ is in $Y^{\lambda, \tau}\left(\mathbb{F}^{\prime}\right)$, it is semisimple with an admissible shape $\widetilde{w} \in$ $\operatorname{Adm}^{\vee}(\lambda)$. 
Now suppose that $\tau$ is 3-generic, but $\bar{\rho}$ is not necessarily a direct sum of characters. In this case, the Kisin module $\overline{\mathfrak{M}}$ of type $(\eta, \tau)$ is unique by [LLHLM18, Theorem 3.2]. We make a base change to the unramified extension $\breve{K} / K$ of degree 6 . Let $\breve{\tau}$ be the base change of $\tau$ to $\breve{K}$, and let $\breve{\mathfrak{M}}=\mathrm{BC}(\overline{\mathfrak{M}})$ be the base change of the Kisin module $\overline{\mathfrak{M}}$. Since $\breve{\bar{M}}$ is the unique Kisin module of type $(\eta, \breve{\tau})$, by the above argument, it must be semisimple.

Recall the notion of gauge basis [LLHLM18, Definition 2.22]. Fix a gauge basis $\beta$ of $\overline{\mathfrak{M}}$ and let $\breve{\beta}$ be the induced gauge basis on $\breve{\mathfrak{M}}$. The eigenbasis $\breve{\beta}_{2}$ for $\breve{\mathfrak{M}}$ which puts the partial Frobenii in the form as in Proposition 3.3.6 is also a gauge basis. By [LLHLM18, Theorem 4.16], $\breve{\beta}$ and $\breve{\beta}_{2}$ differ by embeddingwise scaling by torus elements. We conclude that the matrices for the partial Frobenii with respect to $\breve{\beta}$ and hence $\beta$ are monomial. Since the only monomial matrices $\mathcal{I}(\mathbb{F}) \widetilde{w} \mathcal{I}(\mathbb{F})$ are $T(\mathbb{F}[[v]]) \widetilde{w}$, we see that $\overline{\mathfrak{M}}$ is semisimple.

3.4. Shapes and Serre weights. We continue to assume $\mathcal{S}=\{\widetilde{v}\}$. We compute $V\left(\left.\bar{\rho}\right|_{I_{K}}\right)$ in terms of shape for a semisimple $\bar{\rho}$. This will effectively allow us to determine $W^{?}(\bar{\rho}) \cap \mathrm{JH}(\overline{\sigma(\tau)})$ for a 3-generic tame type $\tau$ (Proposition 3.4.2) via the combinatorics of Section 2 (especially Corollary 2.3.11).

Proposition 3.4.1. Let $(s, \mu)$ be a lowest alcove presentation of $\tau$. Let $\overline{\mathfrak{M}} \in$ $Y^{[0, h], \tau}\left(\mathbb{F}^{\prime}\right)$ be semisimple of shape $\widetilde{w}=\left(\widetilde{w}_{j}\right) \in \underline{\widetilde{W}}^{\vee}$. Then

$$
V\left(\left.T_{\mathrm{dd}}^{*}(\overline{\mathfrak{M}})\right|_{I_{K}}\right)=R_{s \widetilde{w}^{*}}(\mu+\eta) .
$$

Proof. By Proposition 3.3.8, $\left.T_{\mathrm{dd}}^{*}(\overline{\mathfrak{M}})\right|_{I_{K}} \cong \bar{\tau}(w, v+\eta)$ where $\widetilde{w} s^{*} t_{\mu^{*}}=w^{*} t_{v^{*}}$. By [GHS18, Proposition 9.2.3],

$$
V\left(\left.T_{\mathrm{dd}}^{*}(\overline{\mathfrak{M}})\right|_{I_{K}}\right)=R_{w}(v+\eta) .
$$

Finally, an easy calculation shows that $R_{s \widetilde{w}^{*}}(\mu+\eta)=R_{w}(v+\eta)$ using Definition 2.3.6.

Proposition 3.4.2. Let $\tau$ be a 3-generic type with lowest alcove presentation $(s, \mu)$ and let $\overline{\mathfrak{M}} \in Y^{[0, h], \tau}\left(\mathbb{F}^{\prime}\right)$ be semisimple of shape $\widetilde{w}=\left(\widetilde{w}_{j}\right) \in \underline{W}^{\vee}$. Let $\bar{\rho}:$ $G_{K} \rightarrow \mathrm{GL}_{3}(\mathbb{F})$ be semisimple and 3-generic and assume that $\left.\bar{\rho}\right|_{G_{K_{\infty}}} \cong T_{\mathrm{dd}}^{*}(\overline{\mathfrak{M}})$. Then

$$
W^{?}(\bar{\rho}, \tau) \stackrel{\text { def }}{=} W^{?}(\bar{\rho}) \cap \mathrm{JH}(\overline{\sigma(\tau)})=\left\{\sigma_{(\omega, a)}^{(s, \mu+\eta)}:(\omega, a) \in \Sigma_{\widetilde{w}^{*}}\right\} \subset \mathrm{JH}\left(\overline{R_{s}(\mu+\eta)}\right) .
$$

Proof. This follows from Corollary 2.3.11 and Proposition 3.4.1. 
REMARK 3.4.3. There is an explicit list of elements of $\operatorname{Adm}^{\vee}(2,1,0)$ given in [LLHLM18, Table 1]. The effect of the involution $\widetilde{w} \mapsto \widetilde{w}^{*}$ is, in addition, to reverse the order of components, to reverse the order of the word, and to turn $\gamma=(13) v^{(1,0,-1)}$ into $\gamma^{+}$. In particular, $(\cdot)^{*}$ defines a bijection between $\operatorname{Adm}^{\vee}(2$, $1,0)$ and $\operatorname{Adm}(2,1,0)$.

3.4.1. Type elimination results. We assume throughout that $\bar{\rho}: G_{K} \rightarrow \mathrm{GL}_{3}(\mathbb{F})$ is 6 -generic.

PROPOSITION 3.4.4. Let $\tau$ be a 1-generic tame inertial type. If $\bar{\rho}$ is 6-generic and arises as the reduction of a potentially crystalline representation of type $(\eta, \tau)$, then

$$
W^{?}\left(\bar{\rho}^{\mathrm{ss}}, \tau\right) \neq \emptyset \text {. }
$$

Proof. First, assume that $\tau$ is 3-generic. By Theorem 3.3.11 combined with Lemma 3.3.10, there exists $\overline{\mathfrak{M}} \in Y^{\eta, \tau}(\mathbb{F})$ such that $\left.T_{\mathrm{dd}}^{*}(\overline{\mathfrak{M}}) \cong \bar{\rho}^{\mathrm{ss}}\right|_{G_{K_{\infty}}}$. In fact, by Theorem 3.3.12, we can take $\overline{\mathfrak{M}}$ to be semisimple of shape $\widetilde{w}=\left(\widetilde{w}_{j}\right) \in \operatorname{Adm}^{\vee}(\eta)$. By Proposition 3.4.2 and Table 2, we conclude that

$$
W^{?}\left(\bar{\rho}^{\mathrm{ss}}, \tau\right) \neq \emptyset \text {. }
$$

If $\tau$ is not 3-generic, then Proposition 3.4.5 shows that $\bar{\rho}$ does not arise as the reduction of potentially crystalline representation of type $(\eta, \tau)$ for any such $\tau$.

PROPOSITION 3.4.5. Let $n \geqslant 4$ and assume that $\bar{\rho}: G_{K} \rightarrow \mathrm{GL}_{3}(\mathbb{F})$ is n-generic. Assume that $\bar{\rho}$ arises as the reduction of a potentially crystalline representation of type $(\eta, \tau)$ where $\tau$ is a 1-generic tame inertial type. Then $\tau$ is $(n-3)$-generic.

Proof. By Lemma 3.3.10, we can assume that $\bar{\rho}$ is semisimple. The result follows now from [LLHL19, Proposition 3.3.2].

3.5. Serre weight conjectures. We are now ready to prove an abstract version of the Serre weight conjecture as well as a numerical Breuil-Mézard statement. In this section, we allow $\mathcal{S}$ to have arbitrary (finite) cardinality.

3.5.1. Setup and summary of results. Recall from Section 1.4 that $\mathcal{S}$ is a finite set and $F_{\widetilde{v}}$ is a finite extension of $\mathbb{Q}_{p}$ for each $\widetilde{v} \in \mathcal{S}$, and $k_{\widetilde{v}}$ is the residue field of $F_{\widetilde{v}}$. For Definition 3.5.1, we will not require that $F_{\widetilde{v}}$ be an unramified extension of $\mathbb{Q}_{p}$. In applications, $F$ will be a fixed global field, $\mathcal{S}$ will be a set of places in 
$F$, and $F_{\widetilde{v}}$ will be the completion at $\widetilde{v}$ of $F$. Let $\bar{\rho}_{\mathcal{S}}$ be a collection $\left(\bar{\rho}_{\widetilde{v}}\right)_{\widetilde{v} \in \mathcal{S}}$ where $\bar{\rho}_{\widetilde{v}}: G_{F_{\widetilde{v}}} \rightarrow \mathrm{GL}_{3}(\mathbb{F})$ is a continuous Galois representation. Let $R_{\widetilde{v}}^{\square}=R_{\bar{\rho}_{\widetilde{v}}}^{\square}$ denote the unrestricted universal framed deformation ring of $\bar{\rho}_{\widetilde{v}}$. Fix a natural number $h$ and let

$$
R_{\infty}=\left(\widehat{\bigotimes}_{\widetilde{v} \in \mathcal{S}} R_{\widetilde{v}}^{\square}\right) \llbracket x_{1}, x_{2}, \ldots, x_{h} \rrbracket \quad \text { and } \quad X_{\infty}=\operatorname{Spf} R_{\infty} .
$$

If $\tau_{\widetilde{v}}$ is an inertial type for $G_{F_{\widetilde{v}}}$, then let $R_{\widetilde{v}}^{\tau_{v}}=R_{\bar{\rho}_{\widetilde{v}}}^{\tau_{\widetilde{v}}}$ be the universal framed deformation ring of $\bar{\rho}_{\widetilde{v}}$ of inertial type $\tau_{\widetilde{v}}$ and (parallel) Hodge-Tate weights (2, $1,0)$. If $\tau_{\mathcal{S}}=\left(\tau_{\widetilde{v}}\right)_{\tilde{v} \in \mathcal{S}}$, then let

$$
R_{\infty}\left(\tau_{\mathcal{S}}\right)=\widehat{\bigotimes}_{\widetilde{v} \in \mathcal{S}} R_{\widetilde{v}}^{\tau_{\tilde{v}}} \otimes_{\widehat{\otimes} \in \mathcal{S}} R_{\widetilde{v}} R_{\infty} \quad \text { and } \quad X_{\infty}\left(\tau_{\mathcal{S}}\right)=\operatorname{Spf} R_{\infty}\left(\tau_{\mathcal{S}}\right)
$$

Let $d+1$ be the dimension of $X_{\infty}\left(\tau_{\mathcal{S}}\right)$ (the dimension is independent of $\tau_{\mathcal{S}}$ by [Kis08, Theorem 3.3.4]). We denote by $\bar{R}_{\widetilde{v}}^{\square}, \bar{R}_{\infty}$, and so on, the reduction of these objects modulo $\varpi$.

Let $K_{\widetilde{v}}$ be the group $\mathrm{GL}_{3}\left(\mathcal{O}_{F_{\widetilde{v}}}\right)$ and $K$ be the product $\prod_{\widetilde{v} \in \mathcal{S}} K_{\widetilde{v}}$. Results toward the inertial local Langlands correspondence (cf. Proposition 2.2.6(2)) associate a $\overline{\mathbb{Q}}_{p}$-valued $K_{\widetilde{v}}$-representation $\sigma\left(\tau_{\widetilde{v}}\right)$ to a 1 -generic tame inertial type $\tau_{\widetilde{v}}$ (and $\sigma\left(\tau_{\widetilde{v}}\right)$ can be realized over $\left.\mathcal{O}\right)$.

Definition 3.5.1. Let $\operatorname{Rep}_{K}(\mathcal{O})$ denote the category of continuous $K$ representations over finitely generated $\mathcal{O}$-modules and $\operatorname{Mod}\left(X_{\infty}\right)$ the category of coherent sheaves over $X_{\infty}$.

A weak minimal patching functor for $\bar{\rho}_{\mathcal{S}}=\left(\bar{\rho}_{\widetilde{v}}\right)_{\tilde{v} \in \mathcal{S}}$ is defined to be a nonzero covariant exact functor $M_{\infty}: \operatorname{Rep}_{K}(\mathcal{O}) \rightarrow \operatorname{Mod}\left(X_{\infty}\right)$ satisfying the following axioms:

(1) For each $\widetilde{v} \in \mathcal{S}$, let $\tau_{\widetilde{v}}$ be an inertial type for $G_{F_{v}}$. Let $\sigma\left(\tau_{\mathcal{S}}\right)$ be the $K$-representation $\bigotimes_{\tilde{v} \in \mathcal{S}} \sigma\left(\tau_{\tilde{v}}\right)$. If $\sigma\left(\tau_{\mathcal{S}}\right)^{\circ}$ is an $\mathcal{O}$-lattice in $\sigma\left(\tau_{\mathcal{S}}\right)$, then $M_{\infty}\left(\sigma\left(\tau_{\mathcal{S}}\right)^{\circ}\right)$ is $p$-torsion-free and is maximally Cohen-Macaulay over $R_{\infty}\left(\tau_{\mathcal{S}}\right)$.

(2) If $M_{\infty}\left(R_{1}(\mu)\right)$ is nonzero, then $\bar{\rho}_{\widetilde{v}}$ has a semistable lift of type $\tau_{\widetilde{v}}\left(1, \mu_{\widetilde{v}}\right)$ for all $\widetilde{v} \in \mathcal{S}$.

(3) If $\sigma$ is an irreducible $\prod_{\widetilde{v} \in \mathcal{S}} \mathrm{GL}_{3}\left(k_{\widetilde{v}}\right)$-representation over $\mathbb{F}$, the module $M_{\infty}(\sigma)$ is either 0 or maximal Cohen-Macaulay over its support, which is equidimensional of dimension $d$.

(4) The locally free sheaf $M_{\infty}\left(\sigma\left(\tau_{\mathcal{S}}\right)^{\circ}\right)[1 / p]$ (being maximal CohenMacaulay over the regular ring $\left.R_{\infty}\left(\tau_{\mathcal{S}}\right)\left[\frac{1}{p}\right]\right)$ has rank at most one on each connected component. 
Assume that a weak minimal patching functor $M_{\infty}$ for $\bar{\rho}_{\mathcal{S}}$ exists. Following [GHS18, Definition 3.2.6], let $W^{\mathrm{BM}}\left(\bar{\rho}_{\mathcal{S}}\right)$ be the set of irreducible Grepresentations (recall that $\mathrm{G} \stackrel{\text { def }}{=} \prod_{\widetilde{v} \in \mathcal{S}} \mathrm{GL}_{3}\left(k_{\widetilde{v}}\right)$ ) $\sigma$ over $\mathbb{F}$ such that $M_{\infty}(\sigma)$ is nonzero (note that a priori this set depends on the choice of $M_{\infty}$ ). For a finitely generated $R_{\infty}$-module $M$ with scheme-theoretic support Spec $A$ of dimension at most $d$, define $e(M)$ to be $d$ ! times the coefficient of the degree $d$ term of the Hilbert polynomial of $M$ (considered as an $A$-module). In particular, $e(M)$ is the Hilbert-Samuel multiplicity of $M$ as an $A$ module when $\operatorname{dim} A=d$ and is 0 otherwise.

We now assume that for all $\widetilde{v} \in \mathcal{S}, F_{\widetilde{v}}$ is an unramified extension of $\mathbb{Q}_{p}$. Recall Definition 2.2.7 of $W^{?}\left(\bar{\rho}_{\mathcal{S}}\right)$.

THEOREM 3.5.2. Suppose that $\bar{\rho}_{\mathcal{S}}$ is semisimple and 10-generic and that $M_{\infty}$ is a weak patching functor for $\bar{\rho}_{\mathcal{S}}$. Then for all Serre weights $\sigma, e\left(M_{\infty}(\sigma)\right)=1$ if $\sigma \in W^{?}\left(\bar{\rho}_{\mathcal{S}}\right)$ and $e\left(M_{\infty}(\sigma)\right)=0$ otherwise. In particular, $W^{\mathrm{BM}}\left(\bar{\rho}_{\mathcal{S}}\right)=W^{?}\left(\bar{\rho}_{\mathcal{S}}\right)$.

THEOREM 3.5.3. Let $K / \mathbb{Q}_{p}$ be a finite unramified extension of degree $f$. Let $\bar{\rho}: G_{K} \rightarrow \mathrm{GL}_{3}(\mathbb{F})$ be a continuous, 10-generic, and semisimple Galois representation, and let $\tau$ be a tame inertial type. If $\tau$ is not 1-generic, $R_{\bar{\rho}}^{\tau}$ is 0 . If $\tau$ is 1-generic, then the number of irreducible components of $\bar{R}_{\bar{\rho}}^{\tau} \stackrel{\text { def }}{=} R_{\bar{\rho}}^{\tau} / \varpi$ is equal to the number of elements in $W^{?}(\bar{\rho}, \tau)$. The ring $R_{\bar{\rho}}^{\tau}$ is a normal domain and is Cohen-Macaulay. Moreover, $\bar{R}_{\bar{\rho}}^{\tau}$ is reduced and its components are formally smooth of the same dimension.

The proofs of Theorems 3.5.2 and 3.5.3 appear in Section 3.5.3.

3.5.2. Types, weights, and the Breuil-Mézard philosophy. In this subsection, we describe the basic inductive argument toward the proofs of Theorems 3.5.2 and 3.5.3.

Recall that we have a length function $\ell: \widetilde{W}^{\vee} \rightarrow \mathbb{Z}$.

LEMMA 3.5.4. Let $K / \mathbb{Q}_{p}$ be a finite unramified extension of degree $f$. Let $\bar{\rho}: G_{K} \rightarrow \mathrm{GL}_{3}(\mathbb{F})$ be a semisimple Galois representation. Let $\tau$ be 5-generic with lowest alcove presentation $(s, \mu)$. Assume that $V\left(\left.\bar{\rho}\right|_{I_{K}}\right)=R_{s \widetilde{w}^{*}}(\mu+\eta)$ where $\widetilde{w}=\widetilde{w}(\bar{\rho}, \tau)=\left(\widetilde{w}_{0}, \ldots, \widetilde{w}_{f-1}\right) \in \operatorname{Adm}^{\vee}(\eta)$ with $\ell\left(\widetilde{w}_{j}\right)>1$ for every $j$. Then $\bar{R}_{\bar{\rho}}^{\tau} \neq 0$ and the number of irreducible components of $\bar{R}_{\bar{\rho}}^{\tau}$ is equal to the number of elements in $W^{?}(\bar{\rho}, \tau)$. The ring $R_{\bar{\rho}}^{\tau}$ is a normal domain and is CohenMacaulay. Moreover, $\bar{R}_{\bar{\rho}}^{\tau}$ is reduced and its components are formally smooth of the same dimension. 
Proof. By Proposition 3.4.1, our hypotheses imply that there exists a Kisin module $\overline{\mathfrak{M}}_{\bar{\rho}} \in Y^{\eta, \tau}(\mathbb{F})$ such that $\left.T_{\mathrm{dd}}^{*}\left(\overline{\mathfrak{M}}_{\bar{\rho}}\right) \cong \bar{\rho}\right|_{G_{K_{\infty}}}$ and that $\widetilde{w}=\widetilde{w}(\bar{\rho}, \tau)$. In [LLHLM18, Sections 5.3,6], we gave an explicit presentation for $R_{\bar{\rho}}^{\tau}$ whenever the length of $\widetilde{w}_{j}$ is at least 2 and the type $\tau$ is 5-generic. For each shape, there were at most two cases depending on $\overline{\mathfrak{M}}_{\bar{\rho}}$. By Propositions 3.3.9 and 3.3.12, whenever $\bar{\rho}$ is semisimple, $\overline{\mathfrak{M}}_{\bar{\rho}}$ is semisimple in the sense of Definition 3.3.1, and so $\overline{\mathfrak{M}}_{\bar{\rho}}$ lies in the special locus for the given shape.

We claim that

$$
\# \operatorname{Irr}\left(\operatorname{Spec}\left(\bar{R}_{\bar{\rho}}^{\tau}\right)\right)=\prod_{j} 2^{4-\ell\left(\widetilde{w}_{j}\right)}
$$

and that $\bar{R}_{\bar{\rho}}^{\tau}$ is reduced and Cohen-Macaulay. Indeed, from Table 7 in loc. cit., we see that $\bar{R}_{\bar{\rho}}^{\tau}$ is the completion of a tensor product over $\mathbb{F}$ of rings of the form $\mathbb{F}[X], \mathbb{F}[X, Y] / X Y$ at the maximal ideal generated by the variables $X, Y$. Since such a tensor product is Cohen-Macaulay, so is its completion by [Sta19, Tag 07NX]. Since such a tensor product is reduced and excellent (being a finite type $\mathbb{F}$-algebra), its completion is reduced by [Sta19, Tag 07NZ]. Finally, we also note that since each irreducible components of such a tensor product is smooth, the completion of the irreducible components stay irreducible and are formally smooth.

This implies the remaining properties (see the proof of [LLHLM18, Corollary 8.9]. Note that the reducedness of $\bar{R}_{\bar{\rho}}^{\tau}$ implies that $R_{\bar{\rho}}^{\tau}$ is a normal domain).

Finally, we compare this with the size of $W^{?}(\bar{\rho}, \tau)$, which is $\prod_{j \in \mathcal{J}} \# \Sigma_{\left(\widetilde{w}_{j}^{*}\right)}$ by Proposition 3.4.2 using Table 2.

COROLlary 3.5.5. Suppose that for each $\widetilde{v} \in \mathcal{S}, \bar{\rho}_{\widetilde{v}}: G_{F_{\tilde{v}}} \rightarrow \mathrm{GL}_{3}(\mathbb{F})$ is a semisimple Galois representation and $\tau_{\widetilde{v}}$ is a 5-generic tame inertial type satisfying the hypotheses of Lemma 3.5.4. Let $\tau_{\mathcal{S}}$ be $\left(\tau_{\widetilde{v}}\right)_{\widetilde{v} \in \mathcal{S}}$. Then $e\left(\bar{R}_{\infty}\left(\tau_{\mathcal{S}}\right)\right)$ is equal to $\# W^{?}\left(\bar{\rho}_{\mathcal{S}}, \tau_{\mathcal{S}}\right)$.

Proof. This follows immediately from Lemma 3.5.4 and [EG14, Lemma 2.2.14] and properties of tensor products of representations.

Proposition 3.5.6 (Weight elimination). Suppose that for each $\widetilde{v}, \bar{\rho}_{\widetilde{v}}: G_{F_{\widetilde{v}}} \rightarrow$ $\mathrm{GL}_{3}(\mathbb{F})$ is a 10-generic Galois representation. Then

$$
W^{\mathrm{BM}}\left(\bar{\rho}_{\mathcal{S}}\right) \subset W^{?}\left(\bar{\rho}_{\mathcal{S}}^{\mathrm{ss}}\right) .
$$

Proof. Suppose that $\sigma$ is a Serre weight such that $\sigma \in W^{\mathrm{BM}}\left(\bar{\rho}_{\mathcal{S}}\right) \backslash W^{?}\left(\bar{\rho}_{\mathcal{S}}^{\mathrm{ss}}\right)$. Note that $W^{\mathrm{BM}}\left(\bar{\rho}_{\mathcal{S}}\right)$ satisfies the (evident generalization of) [Enn, Axiom (WE)] 
by 3.5.1(2). By [Enn, Theorem 8] and [LLHL19, Remark 2.2.8], $\bar{\rho}_{\mathcal{S}}$ is 10generic implies that $\sigma$ is 3-generic. Then $\sigma \in \mathrm{JH}\left(\overline{\sigma\left(\tau_{\mathcal{S}}\right)}\right)$ for some collection $\tau_{\mathcal{S}} \stackrel{\text { def }}{=}\left(\tau_{\widetilde{v}}\right)_{\tilde{v} \in \mathcal{S}}$ of 1-generic tame types (for example, taking a tame principal series type containing $\sigma$ ). Since $\bar{\rho}_{\mathcal{S}}$ is 10-generic, we see by Proposition 3.4.5 and Definition 3.5.1(1) that $M_{\infty}\left(\overline{\sigma\left(\tau_{\mathcal{S}}\right)}\right) \neq 0$ implies that $\tau_{\mathcal{S}}$ is 7-generic. By Lemma 4.2.13 (whose proof only uses modular representation theory and is independent of the results in Section 3), $\sigma$ is 5-deep.

By Corollary $2.3 .13, \tau_{\mathcal{S}}$ above can be taken so that $W^{?}\left(\bar{\rho}_{\mathcal{S}}^{\mathrm{ss}}, \tau_{\mathcal{S}}\right)=\emptyset$ and $\sigma \in \mathrm{JH}\left(\overline{\sigma\left(\tau_{\mathcal{S}}\right)}\right)$. By Proposition 3.4.4, $\bar{\rho}_{\widetilde{v}}^{\text {ss }}$ is not the reduction of a potentially crystalline representation of type $\left(\eta_{\widetilde{v}}, \tau_{\widetilde{v}}\right)$ for some $\widetilde{v} \in \mathcal{S}$. By Proposition 3.3.10, $\bar{\rho}_{\widetilde{v}}$ is also not the reduction of a potentially crystalline representation of type $\left(\eta_{\tilde{v}}\right.$, $\left.\tau_{\tilde{v}}\right)$ for some $\tilde{v} \in \mathcal{S}$. Then $X_{\infty}\left(\tau_{\mathcal{S}}\right)=\emptyset$ and $M_{\infty}\left(\sigma\left(\tau_{\mathcal{S}}\right)^{\circ}\right)=0$ for any $\mathcal{O}$-lattice $\sigma\left(\tau_{\mathcal{S}}\right)^{\circ}$ in $\sigma\left(\tau_{\mathcal{S}}\right)$. By exactness of $M_{\infty}, M_{\infty}(\sigma)=0$ which is a contradiction.

REMARK 3.5.7. Instead of appealing to [Enn], one can show that provided that $\bar{\rho}_{\mathcal{S}}$ is generic enough, every element of $W^{\mathrm{BM}}\left(\bar{\rho}_{\mathcal{S}}\right)$ appears as a Jordan-Hölder factor of the reduction of some 1-generic tame type as follows. Even though not all Serre weights come from the reduction $\sigma\left(\tau_{\mathcal{S}}\right)$ for a collection $\tau_{\mathcal{S}}$ of 1generic tame types, they do occur in the reduction of $V_{\lambda} \otimes \sigma\left(\tau_{\mathcal{S}}\right)$ where $\lambda$ is sufficiently large (independent of $p$ ). The results of Section 3.3 hold also for potentially crystalline representations of type $\left(\lambda_{\widetilde{v}}+\eta_{\widetilde{v}}, \tau_{\widetilde{v}}\right) \tilde{v}_{\in \mathcal{S}}$ (possibly with a stronger genericity hypothesis), and then the same argument as in the proof of Proposition 3.5.6 gives the result.

Given a collection $\tau_{\mathcal{S}}$ of tame inertial types and a $\mathcal{O}$-lattice $\sigma\left(\tau_{\mathcal{S}}\right)^{\circ}$ in $\sigma\left(\tau_{\mathcal{S}}\right)$, we write $\bar{\sigma}\left(\tau_{\mathcal{S}}\right)^{\circ}$ to denote the reduction of $\sigma\left(\tau_{\mathcal{S}}\right)^{\circ}$ modulo $\varpi$.

LEMMA 3.5.8. Suppose that for each $\widetilde{v} \in \mathcal{S}, \bar{\rho}_{\widetilde{v}}: G_{F_{\widetilde{v}}} \rightarrow \mathrm{GL}_{3}(\mathbb{F})$ is a semisimple Galois representation and $\tau_{\widetilde{v}}$ is a 5-generic tame inertial type satisfying the hypotheses of Lemma 3.5.4. Let $\tau_{\mathcal{S}}=\left(\tau_{\widetilde{v}}\right)_{\widetilde{v} \in \mathcal{S}}$. Let $\sigma\left(\tau_{\mathcal{S}}\right)^{\circ}$ be an $\mathcal{O}$-lattice in $\sigma\left(\tau_{\mathcal{S}}\right)$. Then either $M_{\infty}\left(\bar{\sigma}\left(\tau_{\mathcal{S}}\right)^{\circ}\right)=0$ or $e\left(M_{\infty}\left(\bar{\sigma}\left(\tau_{\mathcal{S}}\right)^{\circ}\right)\right)$ is equal to e $\left(\bar{R}_{\infty}\left(\tau_{\mathcal{S}}\right)\right)$, and the alternative does not depend on the lattice.

Proof. The generic fiber of $X_{\infty}\left(\tau_{\mathcal{S}}\right)$ is irreducible since its special fiber is reduced (see Lemma 3.5.4). Then since $M_{\infty}\left(\sigma\left(\tau_{\mathcal{S}}\right)^{\circ}\right)$ is maximal CohenMacaulay over $X_{\infty}\left(\tau_{\mathcal{S}}\right)$, either $M_{\infty}\left(\sigma\left(\tau_{\mathcal{S}}\right)^{\circ}\right)$ has full support on $X_{\infty}\left(\tau_{\mathcal{S}}\right)$ or $M_{\infty}\left(\sigma\left(\tau_{\mathcal{S}}\right)^{\circ}\right)$ is 0 . The lemma now follows from the proof of [LLHLM18, Proposition 7.14]. 
LEMMA 3.5.9. Suppose that $\bar{\rho}_{\widetilde{v}}: G_{F_{\widetilde{v}}} \rightarrow \mathrm{GL}_{3}(\mathbb{F})$ is semisimple and 7-generic for all $\widetilde{v} \in \mathcal{S}$. If $\sigma \in W^{?}\left(\bar{\rho}_{\mathcal{S}}\right)$ has defect $\delta$, then there are tame inertial types $\tau_{\widetilde{v}}$ and $\tau_{\widetilde{v}}^{\prime}$ satisfying the hypotheses of Corollary 3.5 .5 such that if $\tau_{\mathcal{S}}=\left(\tau_{\widetilde{v}}\right)_{\tilde{v} \in \mathcal{S}}$ and $\tau_{\mathcal{S}}^{\prime}=\left(\tau_{\widetilde{v}}^{\prime}\right)_{\tilde{v} \in \mathcal{S}}$, we have

(1) $\sigma \in W^{?}\left(\bar{\rho}_{\mathcal{S}}, \tau_{\mathcal{S}}\right) \subset W^{?}\left(\bar{\rho}_{\mathcal{S}}, \tau_{\mathcal{S}}^{\prime}\right)$;

(2) $\# W^{?}\left(\bar{\rho}_{\mathcal{S}}, \tau_{\mathcal{S}}\right)=2^{\delta}$ and $\# W^{?}\left(\bar{\rho}_{\mathcal{S}}, \tau_{\mathcal{S}}^{\prime}\right)=2^{\delta+1}$;

(3) all Serre weights in $W^{?}\left(\bar{\rho}_{\mathcal{S}}, \tau_{\mathcal{S}}\right)$ and $W^{?}\left(\bar{\rho}_{\mathcal{S}}, \tau_{\mathcal{S}}^{\prime}\right)$ have defect at most $\delta$ and if $\delta>0, \sigma$ is the unique Serre weight in $W^{?}\left(\bar{\rho}_{\mathcal{S}}, \tau_{\mathcal{S}}^{\prime}\right)$ with defect $\delta$;

(4) if $\delta=0$, for any $\sigma^{\prime} \in W^{?}\left(\bar{\rho}_{\mathcal{S}}\right)$ of defect 0 which is adjacent to $\sigma$, one can choose the $\tau_{\mathcal{S}}^{\prime}$ above so that $W^{?}\left(\bar{\rho}_{\mathcal{S}}, \tau_{\mathcal{S}}^{\prime}\right)=\left\{\sigma, \sigma^{\prime}\right\}$.

Proof. This is essentially a consequence of results in Section 2.3. Suppose that $V\left(\left.\bar{\rho}_{\mathcal{S}}\right|_{I_{F_{\mathcal{S}}}}\right)=R_{S}(\mu)$ with $\mu-\eta$ 7-deep in alcove $\underline{C}_{0}$, and label $W^{?}\left(\bar{\rho}_{\mathcal{S}}\right)$ by $r(\Sigma)$ as in Proposition 2.3.4. Let $\widetilde{w}=\left(\widetilde{w}_{\widetilde{v}}\right)_{\tilde{v} \in \mathcal{S}}=\left(\widetilde{w}_{i}\right)_{i \in \mathcal{J}} \in \operatorname{Adm}(\eta)$ and $\tau_{\mathcal{S}}$ be the tame inertial type with lowest alcove presentation $\left(s w^{-1}, \mu+s \widetilde{w}^{-1}(0)-\eta\right)$, where $w \in \underline{W}$ is the image of $\widetilde{w}$. Then $\mu+s \widetilde{w}^{-1}(0)-\eta$ is 5-deep in alcove $\underline{C}_{0}$ so that $\tau_{\mathcal{S}}$ is 5-generic. Corollary 2.3.11 says that

$$
W^{?}\left(\bar{\rho}_{\mathcal{S}}, \tau_{\mathcal{S}}\right)=\left\{\sigma_{r(\omega, a)}^{(s, \mu)}: r(\omega, a) \in \prod_{i \in \mathcal{J}} r\left(\Sigma_{\widetilde{w}_{i}^{-1}}\right)\right\},
$$

where $\Sigma_{\widetilde{w}_{i}^{-1}}=\Sigma_{0} \cap \widetilde{w}_{i}^{-1}\left(r\left(\Sigma_{0}\right)\right)$. If $\ell\left(\widetilde{w}_{i}\right)>1$ for all $i$, then $\tau_{\mathcal{S}}$ satisfies the hypotheses of Corollary 3.5.5 (noting that $\ell(\widetilde{w})=\ell\left(\widetilde{w}^{*}\right)$ by the proof of [LLHL19, Lemma 2.1.3], where the lengths are as elements of $\underline{\widetilde{W}}^{\vee}$ and $\underline{\widetilde{W}}$, respectively).

Let $(\omega, a)=\left(\left(\omega_{i}, a_{i}\right)\right)_{i}$ be such that $\sigma=\sigma_{r(\omega, a)}^{(s, \mu)}$. We will construct the required types by appropriately choosing $\left(\widetilde{w}_{i}\right)_{i}$.

If $\left(\omega_{i}, a_{i}\right) \in \Sigma_{0}^{\text {obv }}$, we can find an element $\widetilde{w}_{i} \in \operatorname{Adm}((2,1,0))$ such that $\ell\left(\widetilde{w}_{i}\right)=4$ and $\Sigma_{\widetilde{w}_{i}^{-1}}=\left\{\left(\omega_{i}, a_{i}\right)\right\}$. If $\left(\omega_{i}, a_{i}\right) \in \Sigma_{0}^{\text {inn }}$, we can find an element $\widetilde{w}_{i} t_{-1} \in\{\alpha \beta \alpha, \beta \gamma \beta, \alpha \gamma \alpha\}$ such that $\Sigma_{\widetilde{w}_{i}^{-1}}$ contains exactly $\left\{\left(\omega_{i}, a_{i}\right), r\left(\omega_{i}, a_{i}\right)\right\}$. This choice of $\left(\widetilde{w}_{i}\right)_{i \in \mathcal{J}}$ gives a type $\tau_{\mathcal{S}}=\left(\tau_{\widetilde{v}}\right)_{\tilde{v} \in \mathcal{S}}$ such that $\sigma \in W^{?}\left(\bar{\rho}_{\mathcal{S}}, \tau_{\mathcal{S}}\right)$, $\# W^{?}\left(\bar{\rho}_{\mathcal{S}}, \tau_{\mathcal{S}}\right)=2^{\delta}$, and all weights in $W^{?}\left(\bar{\rho}_{\mathcal{S}}, \tau_{\mathcal{S}}\right)$ have defect at most $\delta$.

To construct $\tau_{\mathcal{S}}^{\prime}$ satisfying (2) and (3), we proceed similarly. We first deal with the case $\delta>0$. In this case, we can find a $j_{0} \in \mathcal{J}$ such that $\ell\left(\widetilde{w}_{j_{0}}\right)=3$, and consider any $\widetilde{w}_{j_{0}}^{\prime} \leqslant \widetilde{w}_{j_{0}}$ of length 2 . Then

$$
\Sigma_{\widetilde{w}_{j_{0}}^{-1}} \subset \Sigma_{\widetilde{w}_{j_{0}}^{-1}}
$$




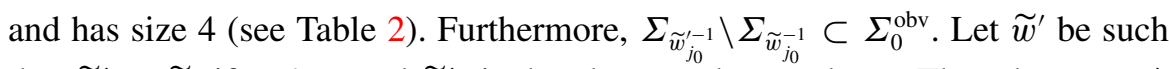
that $\widetilde{w}_{i}^{\prime}=\widetilde{w}_{i}$ if $i \neq j_{0}$ and $\widetilde{w}_{j_{0}}^{\prime}$ is the element chosen above. Then the type $\tau_{\mathcal{S}}^{\prime}$ such that $\sigma\left(\tau_{\mathcal{S}}^{\prime}\right)=R_{s\left(\widetilde{w}^{\prime}\right)^{-1}}(\mu)$ satisfies items (2) and (3).

Finally, assume that $\delta=0$. Let $\sigma^{\prime} \in W^{?}\left(\bar{\rho}_{\mathcal{S}}\right)$ be a defect 0 weight adjacent to $\sigma$, and write $\sigma^{\prime}=\sigma_{r\left(\omega^{\prime}, a^{\prime}\right)}^{(s, \mu)}$. Then there is a unique $j_{0} \in \mathcal{J}$ such that $\left(\omega_{j_{0}}^{\prime}, a_{j_{0}}^{\prime}\right) \neq\left(\omega_{j_{0}}\right.$, $\left.a_{j_{0}}\right)$. There are six possible pairs $\left\{\left(\omega_{j}, a_{j}\right),\left(\omega_{j}^{\prime}, a_{j}^{\prime}\right)\right\} \in \Sigma_{0}^{\text {obv }}$ which are adjacent in Table 1, each of which is $\Sigma_{\widetilde{w}_{j}^{-1}}$ for some length 3 nonshadow element $\widetilde{w}_{j}^{\prime}$ (see [LLHLM18, Table 1]). We let $\tau_{\mathcal{S}}^{\prime}$ be the inertial type corresponding to $\widetilde{w}^{\prime}$ such that $\widetilde{w}_{i}^{\prime}=\widetilde{w}_{i}$ for $i \neq j_{0}$ and $\widetilde{w}_{j_{0}}^{\prime}$ chosen as in the previous sentence. This gives the type satisfying items (2), (3), and (4).

REMARK 3.5.10. From Table 2, we see that the type $\tau_{\mathcal{S}}$ constructed in Lemma 3.5 .9 is uniquely characterized by requiring that $\sigma \in W^{?}\left(\bar{\rho}_{\mathcal{S}}, \tau_{\mathcal{S}}\right)$ and $\# W^{?}\left(\bar{\rho}_{\mathcal{S}}\right.$, $\left.\tau_{\mathcal{S}}\right)=2^{\delta}$. We call it the minimal type of $\sigma$ with respect to $\bar{\rho}$.

In what follows, our $\bar{\rho}_{\mathcal{S}}=\left(\bar{\rho}_{\widetilde{v}}\right)_{\widetilde{v} \in \mathcal{S}}$ will be assumed to be 10 -generic so that Proposition 3.5.6 applies. We observe that if $\tau_{\mathcal{S}}$ is a 3-generic tame type, then $\bar{\sigma}\left(\tau_{\mathcal{S}}\right)^{\circ}$ is multiplicity free for any choice of lattice $\sigma\left(\tau_{\mathcal{S}}\right)^{\circ}$. Then

$$
e\left(M_{\infty}\left(\bar{\sigma}\left(\tau_{\mathcal{S}}\right)^{\circ}\right)\right)=\sum_{\sigma \in \mathrm{JH}\left(\overline{\sigma\left(\tau_{\mathcal{S}}\right)}\right)} e\left(M_{\infty}(\sigma)\right)
$$

by Definition 3.5.1, and, in fact,

$$
e\left(M_{\infty}\left(\bar{\sigma}\left(\tau_{\mathcal{S}}\right)^{\circ}\right)\right)=\sum_{\sigma \in W^{?}\left(\bar{\rho}_{\mathcal{S}}^{\mathrm{ss}}, \tau_{\mathcal{S}}\right)} e\left(M_{\infty}(\sigma)\right) .
$$

Finally, observe that if $\bar{\rho}_{\mathcal{S}}$ is 10 -generic and $W^{?}\left(\bar{\rho}_{\mathcal{S}}, \tau_{\mathcal{S}}\right)$ is nonempty, then $\tau_{\mathcal{S}}$ is 7-generic by Proposition 3.4.5.

LEMma 3.5.11. Suppose that $\bar{\rho}_{\widetilde{v}}: G_{F_{\widetilde{v}}} \rightarrow \mathrm{GL}_{3}(\mathbb{F})$ is semisimple and 10-generic for all $\tilde{v} \in \mathcal{S}$. If there exists $\sigma \in W^{\mathrm{BM}}\left(\bar{\rho}_{\mathcal{S}}\right)$ with defect 0 , then for all $\sigma^{\prime} \in W^{\text {? }}\left(\bar{\rho}_{\mathcal{S}}\right)$, $e\left(M_{\infty}\left(\sigma^{\prime}\right)\right)=1$. In particular, $W^{\mathrm{BM}}\left(\bar{\rho}_{\mathcal{S}}\right)=W^{?}\left(\bar{\rho}_{\mathcal{S}}\right)$.

Proof. We first prove the lemma assuming $\sigma^{\prime}$ has defect 0 by induction on $d \stackrel{\text { def }}{=}$ $\mathrm{d}_{\mathrm{gph}}\left(\sigma, \sigma^{\prime}\right)$. By Lemma 3.5.9, one can choose a 1-generic tame type $\tau_{\mathcal{S}}$ such that $W^{?}\left(\bar{\rho}_{\mathcal{S}}, \tau_{\mathcal{S}}\right)=\{\sigma\}$. Note that $\tau_{\mathcal{S}}$ is then 7-generic under our assumptions. Then $e\left(M_{\infty}(\sigma)\right)=1$ by Corollary 3.5.5 and Lemma 3.5.8. This establishes the case $d=0$.

Suppose that $\sigma^{\prime} \in W^{?}\left(\bar{\rho}_{\mathcal{S}}\right)$ has defect 0 and that $d>0$. Then there is a $\sigma^{\prime \prime} \in W^{?}\left(\bar{\rho}_{\mathcal{S}}\right)$ adjacent to $\sigma^{\prime}$, with defect 0 , and such that $\mathrm{d}_{\mathrm{gph}}\left(\sigma, \sigma^{\prime \prime}\right)=d-1$. 
We choose a type $\tau_{\mathcal{S}}^{\prime}$ as in Lemma 3.5.9(4) for the adjacent weights $\sigma^{\prime}$ and $\sigma^{\prime \prime}$ and an $\mathcal{O}$-lattice $\sigma\left(\tau_{\mathcal{S}}^{\prime}\right)^{\circ}$ in $\sigma\left(\tau_{\mathcal{S}}^{\prime}\right)$. Then by inductive hypothesis, $M_{\infty}\left(\sigma^{\prime \prime}\right)$, and hence $M_{\infty}\left(\bar{\sigma}\left(\tau_{\mathcal{S}}^{\prime}\right)^{\circ}\right)$, is nonzero. By Corollary 3.5.5 and Lemma 3.5.8, $e\left(M_{\infty}\left(\bar{\sigma}\left(\tau_{\mathcal{S}}^{\prime}\right)^{\circ}\right)=2\right.$. We deduce from the inductive hypothesis that $e\left(M_{\infty}\left(\sigma^{\prime}\right)\right)=1$.

We now prove the general case of the lemma by induction on the defect. Suppose that $\sigma^{\prime} \in W^{?}\left(\bar{\rho}_{\mathcal{S}}\right)$ has defect $\delta>0$. We choose $\tau_{\mathcal{S}}$ as in Lemma 3.5.9 (and an $\mathcal{O}$-lattice $\sigma\left(\tau_{\mathcal{S}}\right)^{0}$ in $\sigma\left(\tau_{\mathcal{S}}\right)$ ) taking $\sigma$ to be $\sigma^{\prime}$. By Lemma 3.5.9(2) and (3), $W^{?}\left(\bar{\rho}_{\mathcal{S}}, \tau_{\mathcal{S}}\right)$ contains a weight of lower defect. The inductive hypothesis implies that $M_{\infty}\left(\bar{\sigma}\left(\tau_{\mathcal{S}}\right)^{\circ}\right)$ is nonzero. By Lemma 3.5.8 and Corollary 3.5.5, $e\left(M_{\infty}\left(\bar{\sigma}\left(\tau_{\mathcal{S}}\right)^{\circ}\right)\right)=2^{\delta}$. By Lemma 3.5.9(3) and induction, $e\left(M_{\infty}\left(\bar{\sigma}\left(\tau_{\mathcal{S}}\right)^{\circ}\right)\right)-$ $e\left(M_{\infty}\left(\sigma^{\prime}\right)\right)$ is the number of weights in $W^{?}\left(\bar{\rho}_{\mathcal{S}}, \tau_{\mathcal{S}}\right) \backslash\left\{\sigma^{\prime}\right\}$, which is $2^{\delta}-1$. We conclude that $e\left(M_{\infty}\left(\sigma^{\prime}\right)\right)=1$.

LEMMA 3.5.12. Suppose that $\bar{\rho}_{\widetilde{v}}: G_{F_{\tilde{v}}} \rightarrow \mathrm{GL}_{3}(\mathbb{F})$ is semisimple and 10-generic for all $\tilde{v} \in \mathcal{S}$. If $\sigma \in W^{\mathrm{BM}}\left(\bar{\rho}_{\mathcal{S}}\right)$ has defect $\delta>0$, then there exists $\sigma^{\prime} \in W^{\mathrm{BM}}\left(\bar{\rho}_{\mathcal{S}}\right)$ with defect less than $\delta$.

Proof. Choose $\tau_{\mathcal{S}}$ and $\tau_{\mathcal{S}}^{\prime}$ as in Lemma 3.5.9 and fix lattices $\sigma\left(\tau_{\mathcal{S}}\right)^{\circ}$ and $\sigma\left(\tau_{\mathcal{S}}^{\prime}\right)^{\circ}$. Then $M_{\infty}\left(\sigma\left(\tau_{\mathcal{S}}\right)^{\circ}\right)$ and $M_{\infty}\left(\sigma\left(\tau_{\mathcal{S}}^{\prime}\right)^{\circ}\right)$ are nonzero. Hence, by Lemmas 3.5.8 and 3.5.9(2) and Corollary 3.5.5, $e\left(M_{\infty}\left(\bar{\sigma}\left(\tau_{\mathcal{S}}^{\prime}\right)^{\circ}\right)\right)-e\left(M_{\infty}\left(\bar{\sigma}\left(\tau_{\mathcal{S}}^{\prime}\right)^{\circ}\right)\right)=2^{\delta}$. By Proposition 3.5.9(1), $e\left(M_{\infty}\left(\bar{\sigma}\left(\tau_{\mathcal{S}}^{\prime}\right)^{\circ}\right)\right)-e\left(M_{\infty}\left(\bar{\sigma}\left(\tau_{\mathcal{S}}\right)^{\circ}\right)\right)$ is the sum of $e\left(M_{\infty}\left(\sigma^{\prime}\right)\right)$ as $\sigma^{\prime}$ runs over the Serre weights in $W^{?}\left(\bar{\rho}_{\mathcal{S}}, \tau_{\mathcal{S}}^{\prime}\right) \backslash W^{?}\left(\bar{\rho}_{\mathcal{S}}, \tau_{\mathcal{S}}\right)$. By Lemma 3.5.9(3), the Serre weights in $W^{?}\left(\bar{\rho}_{\mathcal{S}}, \tau_{\mathcal{S}}^{\prime}\right) \backslash W^{?}\left(\bar{\rho}_{\mathcal{S}}, \tau_{\mathcal{S}}\right)$ have defect less than $\delta$. We conclude that there must be a Serre weight $\sigma^{\prime} \in W^{\mathrm{BM}}\left(\bar{\rho}_{\mathcal{S}}\right)$ of defect less than $\delta$.

\subsubsection{Proofs}

Proof of Theorem 3.5.2. Since $M_{\infty}$ is nonzero, there is a Serre weight $\sigma \in$ $W^{\mathrm{BM}}\left(\bar{\rho}_{\mathcal{S}}\right) \subset W^{?}\left(\bar{\rho}_{\mathcal{S}}\right)$ by Proposition 3.5.6. By induction on the defect using Lemma 3.5.12, we can assume, without loss of generality, that the defect of $\sigma$ is 0. The theorem now follows from Lemma 3.5.11.

REMARK 3.5.13. Our axioms for $M_{\infty}$ imply that if $\sigma \in \mathrm{JH}\left(\overline{\sigma\left(\tau_{\mathcal{S}}\right)}\right)$, then the support of $M_{\infty}(\sigma)$ is a (possibly empty) union of irreducible components of $\operatorname{Spec} \bar{R}_{\infty}\left(\tau_{\mathcal{S}}\right)$. As $\operatorname{Spec} R_{\infty}\left(\tau_{\mathcal{S}}\right)$ is the preimage of

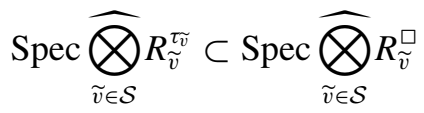


inside Spec $R_{\infty}$, we have shown that if $\sigma \in W^{\mathrm{BM}}\left(\bar{\rho}_{\mathcal{S}}\right)$, then the scheme-theoretic support of $M_{\infty}(\sigma)$ is irreducible with generic point given by the preimage in $R_{\infty}$ of a prime ideal in $\widehat{\bigotimes}_{\widetilde{v} \in \mathcal{S}} R_{\widetilde{v}}^{\square}$.

We now give two examples of weak minimal patching functors using the setup from [LLHLM18, Section 7.1]. Recall the definitions from loc. cit. of $F / F^{+}$, $\Sigma_{p}^{+}, G_{/ F^{+}}$, and $\iota_{w}$ (see also Section 5.3). Suppose that $\bar{r}: G_{F} \rightarrow \mathrm{GL}_{3}(\mathbb{F})$ is

- automorphic (of some weight) in the sense of [LLHLM18, Definition 7.1];

- satisfies the Taylor-Wiles hypotheses in the sense of [LLHLM18, Definition 7.3]; and

- if $\bar{r}$ is ramified at a finite place $w \notin \Sigma_{p}$ of $F$, then $\left.w\right|_{F^{+}}$splits in $F$ (we say that $\bar{r}$ has split ramification outside of $p$ ).

Then [LLHLM18, Proposition 7.15] constructs a weak minimal patching functor for $\bar{r}$ in the sense of [LLHLM18, Definition 7.11], which we will denote by $\widetilde{M}_{\infty}$. Let $h$ be the corresponding integer.

For each $v \in \Sigma_{p}^{+}$, choose a place $\widetilde{v}$ of $F$ lying above $v$. Let $\mathcal{S}_{p}$ be the set $\left\{\widetilde{v}: v \in \Sigma_{p}^{+}\right\}$. Let $\bar{\rho}_{\mathcal{S}_{p}}$ be $\left(\bar{\rho}_{\widetilde{v}}\right)_{\widetilde{v} \in \mathcal{S}_{p}}$ where we define $\bar{\rho}_{\widetilde{v}} \stackrel{\text { def }}{=} \bar{r}_{G_{F_{\tilde{v}}}}: G_{F_{\tilde{v}}} \rightarrow \mathrm{GL}_{3}(\mathbb{F})$. Define $R_{\infty}, R_{\infty}\left(\tau_{\mathcal{S}_{p}}\right)$, and so on, as before with respect to $h$ above. Let $K$ be $\prod_{\mathcal{S}_{p}} K_{\widetilde{v}}$ as before. From the proof of [LLHL19, Proposition 4.2.6] we have the following.

Proposition 3.5.14. Let $M_{\infty}: \operatorname{Rep}_{K}(\mathcal{O}) \rightarrow \operatorname{Mod}\left(X_{\infty}\right)$ be the functor $\tilde{M}_{\infty} \circ$ $\prod_{\widetilde{v} \in \mathcal{S}_{p}} l \widetilde{v}$. Then $M_{\infty}$ is a weak minimal patching functor for $\bar{\rho}_{\mathcal{S}_{p}}$.

Proof. Definition 3.5.1(2) follows from the proof of [LLHL19, Proposition 4.2.6]. The remaining properties follow easily from definitions and [LLHLM18, Proposition 7.15].

Now suppose that $p>3$ and $K / \mathbb{Q}_{p}$ is a finite extension. Let $\bar{\rho}: G_{K} \rightarrow$ $\mathrm{GL}_{3}(\mathbb{F})$ be a continuous Galois representation with a potentially diagonalizable lift of type $(\eta, \tau)$ such that $R_{\bar{\rho}}^{\tau}$ is formally smooth. For example, if $\bar{\rho}$ is 6-generic and semisimple, we can take $\tau$ so that if $\widetilde{w}(\bar{\rho}, \tau)=\left(\widetilde{w}_{j}\right)_{j}$, then $\ell\left(\widetilde{w}_{j}\right)=4$ for all $j$. We now construct a weak minimal patching functor for $\bar{\rho}$ (here $\# \mathcal{S}=1$ in the notation of Definition 3.5.1).

[EG14, Corollary A.7] constructs a CM extension $F / F^{+}$, a choice of places $\mathcal{S}_{p}$ above $\Sigma_{p}^{+}$as before, and an automorphic Galois representation $\bar{r}: G_{F} \rightarrow \mathrm{GL}_{3}(\mathbb{F})$ satisfying the above itemized properties such that there is an isomorphism $K \cong F_{\widetilde{v}}$ and $\left.\bar{r}\right|_{G_{\widetilde{v}}} \cong \bar{\rho}$ for all $\widetilde{v} \in \mathcal{S}_{p}$. Fix a place $\widetilde{v} \in \mathcal{S}_{p}$ 


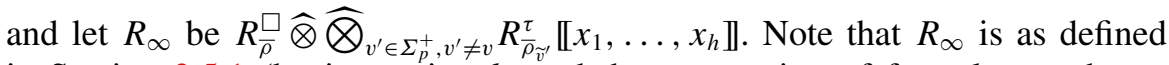
in Section 3.5.1 (by increasing $h$, and the assumption of formal smoothness of $R_{\bar{\rho}}^{\tau}$ ) and we identify $R_{\infty}$ with $R_{\left.\bar{r}\right|_{G_{\widetilde{v}}} ^{\square}}^{\square} \widehat{\otimes} \widehat{\bigotimes}_{v^{\prime} \in \Sigma_{p}^{+}, v^{\prime} \neq v} R_{\left.\bar{r}\right|_{F_{\widetilde{v}^{\prime}}} ^{\tau}}^{\tau} \llbracket x_{1}, \ldots, x_{h} \rrbracket$. Let $X_{\infty}=\operatorname{Spf} R_{\infty}$ as usual. We abusively let $K$ be the group $\operatorname{GL}_{3}\left(\mathcal{O}_{K}\right)$ (the meaning of each $K$ will be clear from the context). Fix a lattice $\sigma(\tau)^{\circ}$ in the $K$-module $\sigma(\tau)$.

Let $\widetilde{M}_{\infty}$ be the weak minimal patching functor for $\bar{r}$ constructed above. The following proposition follows from the construction of $\bar{r}$ and Proposition 3.5.14.

Proposition 3.5.15. Assume the above setup. Let $M_{\infty}: \operatorname{Rep}_{K}(\mathcal{O}) \rightarrow$ $\operatorname{Mod}\left(X_{\infty}\right)$ be the functor

$$
\tilde{M}_{\infty} \circ \prod_{v \in \Sigma_{p}^{+}} \iota_{\widetilde{v}} \circ\left(-\otimes \widehat{\bigotimes}_{v \in \Sigma_{p}^{+}, v^{\prime} \neq v} \sigma(\tau)^{\circ}\right) .
$$

Then $M_{\infty}$ is a patching functor for $\bar{\rho}$.

COROLlary 3.5.16. Suppose that $\bar{\rho}_{\mathcal{S}}$ is a collection of continuous Galois representations satisfying the properties of $\bar{\rho}$ in Proposition 3.5.15. Then there exists a patching functor for $\bar{\rho}_{\mathcal{S}}$.

Proof. We can take the completed tensor product of the patching functors constructed in Proposition 3.5.15 for each $\bar{\rho}_{\widetilde{v}}$.

Proof of Theorem 3.5.3. Suppose that $\tau$ is not 1-generic. We claim that $R_{\bar{\rho}}^{\tau}=$ 0 . It suffices to show that after restriction to $G_{K^{\prime}}$ for any unramified extension $K^{\prime} / K, \bar{\rho}$ does not have a potentially crystalline lift of type $\tau$ and Hodge-Tate weights $(2,1,0)$. Moreover, after such a restriction, $\tau$ is still not 1 -generic and $\bar{\rho}$ is 10-generic. We can then assume, without loss of generality, that $\tau$ is a principal series. By [LLHL19, Remark 2.2.8], $\tau$ is not 2-generic and $\bar{\rho}$ is 10 -generic in the sense of [Enn, Definition 2.2]. Then $\bar{\rho}$ does not have a potentially crystalline lift of type $\tau$ and Hodge-Tate weights $(2,1,0)$ by [Enn, Proposition 7].

If $\tau$ is 1 -generic and $R_{\bar{\rho}}^{\tau} \neq 0$, then Proposition 3.4.5 implies that $\tau$ is 7-generic. Proposition 3.4.2 implies that $W^{?}(\bar{\rho}, \tau) \neq \emptyset$. Suppose that $\tau$ is 1 -generic and $W^{?}(\bar{\rho}, \tau) \neq \emptyset$. By Proposition 3.5.15, a patching functor $M_{\infty}$ for $\bar{\rho}$ exists. For any $\mathcal{O}_{E}$-lattice $\sigma(\tau)^{\circ} \subset \sigma(\tau), M_{\infty}\left(\sigma(\tau)^{\circ}\right) \neq 0$ by Theorem 3.5.2. This implies that $R_{\bar{\rho}}^{\tau}$ is nonzero.

We now show that if $\tau$ is 1 -generic and $R_{\bar{\rho}}^{\tau} \neq 0$, then

$$
e\left(\bar{R}_{\bar{\rho}}^{\tau}\right)=e\left(M_{\infty}\left(\bar{\sigma}(\tau)^{\circ}\right)\right) .
$$


As $R_{\bar{\rho}}^{\tau} \neq 0$, we deduce from Proposition 3.4.5 that $\tau$ is 7-generic. The proof of (3.5) is now obtained by a direct generalization of the arguments of [LLHLM18, Section 8]; so we only explain the key details. There is a ring $\widetilde{R}_{\bar{\rho}}^{\tau}$ of the same dimension as $\bar{R}_{\bar{\rho}}^{\tau} \llbracket X_{1}, \ldots X_{3 f} \rrbracket$ which is a power series ring over the completed tensor product over $\mathbb{F}$ of rings $\widetilde{R}$ in [LLHLM18, Section 8] and $\bar{R} \overline{\mathfrak{M}}, \widetilde{w}$ for some $\overline{\mathfrak{M}}$ and $\widetilde{w}$ from [LLHLM18, Table 7], and which admits a surjection to $\bar{R}_{\bar{\rho}}^{\tau} \llbracket X_{1}$, $\ldots, X_{3 f} \rrbracket$. Then we have

$$
e\left(\widetilde{R}_{\bar{\rho}}^{\tau}\right)=e\left(M_{\infty}\left(\bar{\sigma}(\tau)^{\circ}\right)\right)
$$

for any $\mathcal{O}$-lattice $\sigma(\tau)^{\circ} \subset \sigma(\tau)$ by computing both sides using [LLHLM18, Table 7, Propositions 8.5 and 8.12] and Theorem 3.5.2 (using that $\bar{\rho}$ is 10generic). The proof of [LLHLM18, Proposition 7.14] implies

$$
e\left(M_{\infty}\left(\bar{\sigma}(\tau)^{\circ}\right)\right) \leqslant e\left(\bar{R}_{\bar{\rho}}^{\tau}\right) .
$$

Then we must have that $e\left(\widetilde{R}_{\bar{\rho}}^{\tau}\right)=e\left(\bar{R}_{\bar{\rho}}^{\tau} \llbracket X_{1}, \ldots, X_{3 f} \rrbracket\right)=e\left(\bar{R}_{\bar{\rho}}^{\tau}\right)$ by the above surjection. Furthermore, $\widetilde{R}_{\bar{\rho}}^{\tau}$ is reduced and Cohen-Macaulay by the same argument as in the proof of Lemma 3.5.4. Then $\bar{R}_{\bar{\rho}}^{\tau} \llbracket X_{1}, \ldots, X_{3 f} \rrbracket$ is isomorphic to $\widetilde{R}_{\bar{\rho}}^{\tau}$ by [LLHLM18, Lemma 8.8] and the desired properties of $\bar{R}_{\bar{\rho}}^{\tau}$ hold because they do for $\widetilde{R}_{\bar{\rho}}^{\tau}$. The desired ring-theoretic properties of $R_{\bar{\rho}}^{\tau}$ follow from the proof of [LLHLM18, Corollary 8.9].

REMARK 3.5.17. Since the number of irreducible components of $\bar{R}_{\bar{\rho}}^{\tau}$ is equal to $\# W^{?}(\bar{\rho}, \tau), \bar{R}_{\bar{\rho}}^{\tau}$ is reduced, and $M_{\infty}\left(\bar{\sigma}(\tau)^{\circ}\right)$ has full support over $\bar{R}_{\infty}(\tau)$, the proof of Theorem 3.5.3 shows that the irreducible support of $M_{\infty}(\sigma)$ must be different for each $\sigma \in W^{?}(\bar{\rho}, \tau)$.

3.6. The geometric Breuil-Mézard conjecture. We now show that weak minimal patching functors can be used to assign components in deformation rings to Serre weights.

Proposition 3.6.1. (1) Let $\bar{\rho}$ be as in Theorem 3.5.3. There is a unique assignment $\sigma \mapsto \mathfrak{p}(\sigma)$ for $\sigma \in W^{?}(\bar{\rho})$ such that $\mathfrak{p}(\sigma) \subset R_{\bar{\rho}}^{\square}$ is a prime ideal and

$$
\operatorname{Spec}\left(\bar{R}_{\bar{\rho}}^{\tau}\right)=\bigcup_{\sigma \in W^{?}(\bar{\rho}, \tau)} \operatorname{Spec}\left(R_{\bar{\rho}}^{\square} / \mathfrak{p}(\sigma)\right)
$$

for any tame type $\tau$ where the right-hand side is given the reduced scheme structure. Moreover, the image of $\mathfrak{p}(\sigma)$ in $\bar{R}_{\bar{\rho}}^{\tau}$ is a minimal prime ideal and (3.6) is the decomposition of $\bar{R}_{\bar{\rho}}^{\tau}$ into irreducible components. 
(2) If $M_{\infty}$ is a weak minimal patching functor for $\bar{\rho}_{\mathcal{S}}=\left(\bar{\rho}_{\widetilde{v}}\right)_{\widetilde{v} \in \mathcal{S}}$, then the scheme-theoretic support of $M_{\infty}\left(\bigotimes_{\widetilde{v} \in \mathcal{S}} \sigma_{\widetilde{v}}\right)$ is $\operatorname{Spec}\left(R_{\infty} / \sum_{\widetilde{v} \in \mathcal{S}} \mathfrak{p}\left(\sigma_{\widetilde{v}}\right) R_{\infty}\right)$.

Proof. We first prove uniqueness. Suppose there is such an assignment. This closely follows the procedure of induction on the defect with respect to $W^{?}(\bar{\rho})$ in the proof of Theorem 3.5.2. If the defect of $\sigma \in W^{?}(\bar{\rho})$ is 0 , then by letting $\tau$ be the minimal type of $\sigma$ with respect to $\bar{\rho}$, we have $\# W^{?}(\bar{\rho}, \tau)=1$ by Lemma 3.5.9. Then we must have $\mathfrak{p}(\sigma)=\operatorname{Ann}_{R \square} \bar{R}_{\bar{\rho}}^{\tau}$. If the defect of $\sigma$ is $\delta>0$, then choose $\tau$ to be the minimal type of $\sigma$ with respect to $\bar{\rho}$ as in Lemma 3.5.9. Then by induction and Lemma 3.5.9(3), there is a unique component of $\operatorname{Spec}\left(\bar{R}_{\bar{\rho}}^{\tau}\right)$ whose defining ideal is not $\mathfrak{p}\left(\sigma^{\prime}\right)$ for some $\sigma^{\prime} \in W^{?}(\bar{\rho}, \tau)$ of lower defect. Then $\mathfrak{p}(\sigma)$ must be this defining ideal.

We now show existence of an assignment. By Proposition 3.5.15, there is a weak minimal patching functor $M_{\infty}$ for $\bar{\rho}$, which we fix. By Remark 3.5.13, the generic point of the scheme-theoretic support of $M_{\infty}(\sigma)$ is of the form $\mathfrak{p}^{\prime}(\sigma) R_{\infty}$ for some prime ideal $\mathfrak{p}^{\prime}(\sigma) \subset R_{\bar{\rho}}^{\square}$. We claim that $\sigma \mapsto \mathfrak{p}^{\prime}(\sigma)$ is an assignment satisfying (3.6). Indeed, since the generic fiber of $R_{\bar{\rho}}^{\tau}$ is connected by 3.5.3, $\operatorname{Spec} \bar{R}_{\infty}(\tau)$ is the scheme-theoretic support of $M_{\infty}\left(\bar{\sigma}(\tau)^{\circ}\right)$. On the other hand, $M_{\infty}\left(\bar{\sigma}(\tau)^{\circ}\right)$ is filtered by $M_{\infty}(\sigma)$ for $\sigma \in W^{?}(\bar{\rho}, \tau)$ so that the support of $M_{\infty}\left(\bar{\sigma}(\tau)^{\circ}\right)$ is

$$
\bigcup_{\sigma \in W^{2}(\bar{\rho}, \tau)} \operatorname{Spec}\left(R_{\infty} / \mathfrak{p}^{\prime}(\sigma) R_{\infty}\right)
$$

Equation (3.7) is a decomposition of $\bar{R}_{\infty}(\tau)$ into irreducible components by Remark 3.5.17. Finally, we observe that this statement descends to $\bar{R}_{\bar{\rho}}^{\tau}$.

We now show part (2). Suppose that $M_{\infty}$ is a weak minimal patching functor for $\bar{\rho}_{\mathcal{S}}$. Let $\sigma=\bigotimes_{\widetilde{v} \in \Sigma} \sigma_{\widetilde{v}} \in W^{?}\left(\bar{\rho}_{\mathcal{S}}\right)$. Again by the proof of Theorem 3.5.2, the scheme-theoretic support of $M_{\infty}(\sigma)$ is $\operatorname{Spec} R_{\infty} /\left(\sum_{\widetilde{v} \in \mathcal{S}} \mathfrak{p}(\sigma)_{\widetilde{v}} R_{\infty}\right)$ for some prime ideals $\mathfrak{p}(\sigma)_{\widetilde{v}} \subset R_{\bar{\rho}_{\widetilde{v}}}^{\square}$. We will show that $\mathfrak{p}(\sigma)_{\widetilde{v}}=\mathfrak{p}\left(\sigma_{\widetilde{v}}\right)$, where $\mathfrak{p}$ is the assignment in part (1). We induct on $\delta=\operatorname{Def}_{\bar{\rho}_{\mathcal{S}}}(\sigma)$. If $\delta=0$, then one can choose $\tau_{\mathcal{S}}$ as in Lemma 3.5.9 so that $\bar{R}_{\infty}\left(\tau_{\mathcal{S}}\right)=R_{\infty} / \mathfrak{p}(\sigma)$. We conclude that $\mathfrak{p}(\sigma)_{\widetilde{v}}=\mathfrak{p}\left(\sigma_{\widetilde{v}}\right)$ for all $\widetilde{v} \in \mathcal{S}$ in this case. Suppose that $\delta>0$. Again choose $\tau_{\mathcal{S}}$ as in Lemma 3.5.9. Then by the inductive hypothesis, for any weight $\sigma^{\prime}=$ $\bigotimes_{\tilde{v} \in \mathcal{S}} \sigma_{\widetilde{v}}^{\prime} \in W^{?}\left(\bar{\rho}_{\mathcal{S}}, \tau_{\mathcal{S}}\right)$ with $\sigma^{\prime} ¥ \sigma$, the scheme-theoretic support of $M_{\infty}\left(\sigma^{\prime}\right)$ is $R_{\infty} /\left(\sum_{\widetilde{v} \in \mathcal{S}} \mathfrak{p}\left(\sigma_{\widetilde{v}}^{\prime}\right) R_{\infty}\right)$. Since the generic fiber of Spec $\left(\widehat{\bigotimes}_{\widetilde{v} \in \mathcal{S}} R_{\bar{\rho}_{\widetilde{v}}}^{\tau_{\widetilde{v}}}\right)$ is connected by 3.5.3, by item (1), the scheme-theoretic support of $M_{\infty}\left(\bar{\sigma}\left(\tau_{\mathcal{S}}\right)^{\circ}\right)$ is

$$
\bigcup_{\sigma^{\prime} \in W^{?}\left(\bar{\rho}_{\mathcal{S}}, \tau_{\mathcal{S}}\right)} \operatorname{Spec}\left(R_{\infty} /\left(\sum_{\widetilde{v} \in \mathcal{S}} \mathfrak{p}\left(\sigma_{\widetilde{v}}^{\prime}\right) R_{\infty}\right)\right) .
$$


From this, we see that since $e\left(M_{\infty}(\sigma)\right)=1$, the scheme-theoretic support of $M_{\infty}(\sigma)$ is forced to be $\operatorname{Spec} R_{\infty} /\left(\sum_{\widetilde{v} \in \mathcal{S}} \mathfrak{p}\left(\sigma_{\widetilde{v}}\right) R_{\infty}\right)$.

We have the following refinement of Proposition 3.6.1(2).

LEMMA 3.6.2. Assume that $\bar{\rho}$ is 10-generic. Let $\tau_{\mathcal{S}}=\left(\tau_{\widetilde{v}}\right)_{\widetilde{v} \in \mathcal{S}}$ be a collection of tame inertial types and let $V$ be a subquotient of $\bar{\sigma}\left(\tau_{\mathcal{S}}\right)^{\circ}$ for some $\mathcal{O}$-lattice $\sigma\left(\tau_{\mathcal{S}}\right)^{\circ}$ in $\sigma\left(\tau_{\mathcal{S}}\right)$. Define the closed subscheme $\bar{X}_{\infty}(V) \hookrightarrow \operatorname{Spec} \bar{R}_{\infty}$ to be the reduced subscheme underlying $\bigcup_{\sigma \in \mathrm{JH}(V)} \operatorname{Supp}\left(M_{\infty}(\sigma)\right)$. Then the schemetheoretic support of $M_{\infty}(V)$ is $\bar{X}_{\infty}(V)$. In particular, if $M_{\infty}(V)$ is a cyclic $R_{\infty}$ module, then $M_{\infty}(V) \cong R_{\infty} / I(V)$, where

$$
I(V) \stackrel{\text { def }}{=} \bigcap_{\sigma \in \mathrm{JH}(V)} \operatorname{Ann}_{R_{\infty}}\left(M_{\infty}(\sigma)\right) .
$$

Proof. Since $\bar{\rho}$ is 10 -generic, there is nothing to prove unless $\tau_{S}$ is 7 -generic. The proof now follows exactly as in the second paragraph of the proof of [EGS15, Proposition 8.1.1]. We recall the argument. The support of $M_{\infty}(V)$ is (the topological space) $\bar{X}_{\infty}(V)$ since $M_{\infty}(V)$ is filtered by $M_{\infty}(\sigma)$ for $\sigma \in \mathrm{JH}(V)$. It suffices to show that the scheme-theoretic support of $M_{\infty}(V)$ is reduced. The scheme-theoretic support of $M_{\infty}(V)$ is generically reduced (since the same is true for $\left.M_{\infty}\left(\bar{\sigma}\left(\tau_{\mathcal{S}}\right)^{\circ}\right)\right)$. Now since each $M_{\infty}(\sigma)$ is maximal Cohen-Macaulay over $\bar{R}_{\infty}\left(\tau_{\mathcal{S}}\right)$ (by Definition 3.5.1(3) and the fact that $\operatorname{dim} \bar{R}_{\infty}\left(\tau_{\mathcal{S}}\right)=d$ ) and the maximal Cohen-Macaulay property is preserved under extension (by the characterization of depth in terms of Ext groups), $M_{\infty}(V)$ is maximal CohenMacaulay over $\bar{R}_{\infty}\left(\tau_{\mathcal{S}}\right)$. This guarantees that the scheme-theoretic support of $M_{\infty}(V)$ has no embedded associated primes and, hence, is reduced.

3.6.1. Matching components. Recall that Proposition 3.6.1 gives a canonical parametrization of the irreducible components of the special fiber of the potentially crystalline deformation ring $R_{\bar{\rho}}^{\tau}$ in terms of $W^{?}(\bar{\rho}, \tau)$. Given a lowest alcove presentation $(s, \mu-\eta)$ of $\tau=\tau(s, \mu)$, we will define in item (4) below explicit rings $\bar{R}_{\overline{\mathfrak{M}}, \widetilde{w}}^{\text {expl, },}$, building on [LLHLM18, Sections 5.3.1, 8]. The rings $\bar{R}_{\overline{\mathfrak{M}}, \widetilde{w}}^{\exp , \nabla}$ will be formally smooth modifications of $\bar{R}_{\bar{\rho}}^{\tau}$. Thus, Proposition 3.6.1 gives a bijection between minimal primes of $\bar{R}_{\overline{\mathfrak{M}}, \widetilde{w}}^{\operatorname{exl}, \nabla}$ and $W^{?}(\bar{\rho}, \tau)$. On the other hand, by Corollary 2.3.11, the data $(s, \mu)$ gives a description of $W^{?}(\bar{\rho}$, $\tau)$ as $\sigma_{(\omega, a)}^{(s, \mu)}$, for $(\omega, a) \in \Sigma_{\widetilde{w}}$. In this subsection, we will make the bijection between minimal primes of $\bar{R}_{\overline{\mathfrak{M}}, \widetilde{w}}^{\text {expl, }}$ and $\Sigma_{\widetilde{w}}$ explicit. This will be needed in Section 5 , where we need to check relations between ideals corresponding to various subquotients of $\bar{\sigma}(\tau)^{0}$ for certain lattices $\sigma(\tau)^{0}$. 
We begin by recalling the relationship between $\bar{R}_{\overline{\mathfrak{M}}, \widetilde{w}}^{\operatorname{expl}, \nabla}$ and $\bar{R}_{\bar{\rho}}^{\tau}$. For the rest of this section, we assume that $\bar{\rho}$ is 10-generic. Recall that we have chosen a lowest alcove presentation $(s, \mu-\eta)$ of $\tau=\tau(s, \mu)$. We assume that $R_{\bar{\rho}}^{\tau} \neq 0$, and, thus, $\tau$ is 7 -generic and there exists a unique $\overline{\mathfrak{M}} \in Y^{\eta, \tau}(\mathbb{F})$ such that $T_{\mathrm{dd}}^{*}(\overline{\mathfrak{M}}) \cong$ $\left.\bar{\rho}\right|_{G_{K_{\infty}}}$. Let $\widetilde{w}=\widetilde{w}(\bar{\rho}, \tau)$ be the shape of $\overline{\mathfrak{M}}$ and let $\overline{\mathcal{M}} \stackrel{\text { def }}{=}\left(\overline{\mathfrak{M}}\left[1 / u^{\prime}\right]\right)^{\Delta=1}$. We also recall the notion of gauge basis [LLHLM18, Definitions 4.15, 6.11], and we fix a gauge basis $\bar{\beta}$ for $\overline{\mathfrak{M}}$. We also fix a framing (that is, a basis) for $\bar{\rho}$. Recall from [LLHLM18, Definition 2.11 and Section 6] (see also the discussion after [LLHL19, Definition 3.2.8], which is more aligned with the notation of this paper) the notation $A^{(i)} \stackrel{\text { def }}{=} \operatorname{Mat}_{\beta}\left(\phi_{\mathfrak{M}, s_{\mathrm{or}, i+1}(3)}^{(i)}\right)$, for an eigenbasis $\beta$ of a Kisin module $\mathfrak{M}$ with descent data of type $\tau$ and $s_{\text {or }} \in \underline{W}$ the orientation of $\tau=\tau(s, \mu)$. We say that $A^{(i)}$ is the matrix of the $i$ th partial Frobenius $\phi_{\mathfrak{M}, s_{\mathrm{or}, i+1}(3)}^{(i)}$ with respect to the eigenbasis $\beta$.

We have the following canonical diagram (cf. [LLHLM18, Diagram (5.9)]:

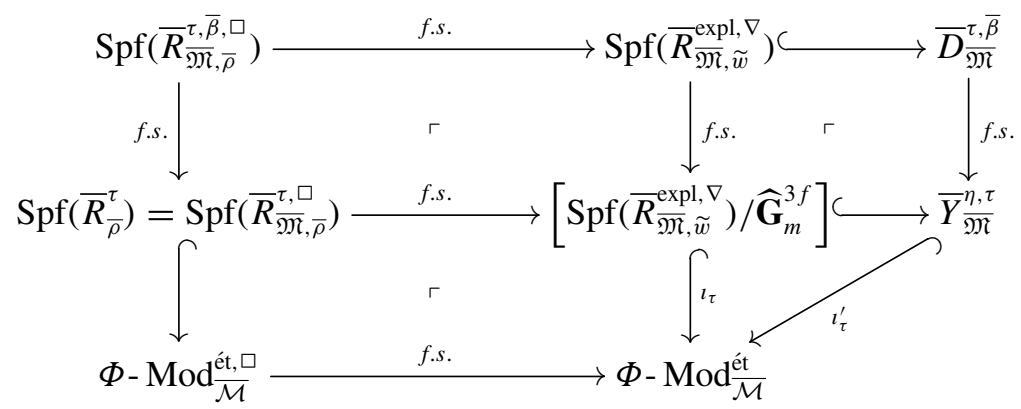

where f.s. stands for a formally smooth morphism. We explain the diagram as follows:

(1) $\bar{R}_{\overline{\mathfrak{M}}, \bar{\rho}, \bar{\beta}}^{\tau, \square}, \bar{R}_{\overline{\mathfrak{M}}, \bar{\rho}}^{\tau, \square}$, are defined in [LLHLM18, Sections 4.3, 5.2, 6.2]. They parametrize various deformation problems of $\bar{\rho}$ and $\overline{\mathfrak{M}}$ with extra data such as framings on Galois representations and gauge bases on $\overline{\mathfrak{M}}$. We note that in loc. cit., we used the symbol $\mu$ for what we call $\eta$ in this paper.

(2) $\Phi$ - $\operatorname{Mod}_{\mathcal{M}}^{\text {ét }}$ (respectively $\Phi$ - $\operatorname{Mod}_{\mathcal{M}}^{\text {ét, } \square}$ ) denotes the groupoid of étale $\varphi$ modules deforming $\overline{\mathcal{M}}$ (respectively deformations with a basis on the associated $G_{K_{\infty}}$-representation).

(3) $\bar{Y} \frac{\eta, \tau}{\mathfrak{M}}$ is the groupoid whose values on a local Artinian $\mathbb{F}$-algebra $A$ is given by the groupoid of pairs $\left(\mathfrak{M}_{A}, J_{A}\right)$ where $\mathfrak{M}_{A} \in Y^{\eta, \tau}(A)$ and $J_{A}: \mathfrak{M}_{A} \otimes_{A}$ $\mathbb{F} \stackrel{\sim}{\rightarrow} \overline{\mathfrak{M}}$ is an isomorphism in $Y^{\eta, \tau}(\mathbb{F})$. The groupoid $\overline{D_{\bar{M}}^{\tau, \bar{\beta}}}$ parametrizes 
the same data plus the data of a gauge basis lifting $\bar{\beta}$. By [LLHLM18, Theorem 6.12], there is an action of $\widehat{\mathbf{G}}_{m}^{3 f}$ on $\overline{D_{\mathfrak{M}}^{\tau, \bar{\beta}}}$ by scaling the gauge basis, and one has $\left[\bar{D}_{\overline{\mathfrak{M}}}^{\tau, \bar{\beta}} / \widehat{\mathbf{G}}_{m}^{3 f}\right] \cong \bar{Y}_{\overline{\mathfrak{M}}}^{\eta, \tau}$. By [LLHLM18, Theorem 4.17 and Section 6.2], $\bar{D} \overline{\mathfrak{M}^{\prime}}$ is representable by $\bar{R} \frac{\overline{\mathcal{M}}}{\overline{\mathfrak{M}}}=\widehat{\bigotimes}_{i}\left(R_{\widetilde{w}_{i}}^{\exp }\right)^{p \text {-flat, red }} / \varpi$. Over $\bar{R} \frac{\tau, \bar{\beta}}{\mathfrak{M}}$, we have a universal pair ( $\left.\mathfrak{M}^{\text {univ }}, \beta^{\text {univ }}\right)$ and, hence, the universal matrices of partial Frobenii $A^{(i) \text {,univ }}$. By construction, $\left(R_{\widetilde{w}_{i}}^{\text {expl }}\right)^{p \text {-flat, red }} / \varpi$ is a quotient of the power series ring over $\mathbb{F}$ generated by (suitable modifications of) the coefficients of the entries of $A^{(i) \text {,univ }}$ subject to certain 'finite height' equations. The map $\imath_{\tau}^{\prime}$ is the map sending $\mathfrak{M}^{\text {univ }}$ to $\left(\mathfrak{M}^{\mathrm{univ}}\left[\frac{1}{\bar{u}^{\prime}}\right]\right)^{\Delta=1}$.

(4) The ring $\bar{R}_{\overline{\mathfrak{M}}, \widetilde{w}}^{\text {expl, }}=\widehat{\bigotimes}_{i} \bar{R}_{\overline{\mathfrak{M}}, \widetilde{w}_{i}}^{\text {exl, }}$. We recall the description of each component ring from [LLHLM18] (see also Table 3). We let $(a, b, c) \in \mathbb{F}_{p}^{3}$ be the $\bmod$ $p$ reduction of $s_{f-1-i}^{-1}\left(\mu_{f-1-i}\right) \in X^{*}(T) \cong \mathbb{Z}^{3}$.

(1) When $\ell\left(\widetilde{w}_{i}\right)>1: \bar{R}_{\overline{\mathfrak{M}}^{\mathrm{w}}, \widetilde{w}_{i}}^{\exp , \nabla}$ is the quotient of the power series ring over $\mathbb{F}$ generated by (suitable modifications of) the coefficients of the entries of $A^{(i) \text {,univ }}$ by an explicit list of relations given by [LLHLM18, Section 5.3 and Table 7]. In this case, we even have the rings $R_{\overline{\mathfrak{M}}, \widetilde{w}_{i}}^{\text {exl, }}$ and diagram (3.9) can be lifted to a diagram over $\mathcal{O}$ with the same properties; cf. [LLHLM18, Diagram (5.9)].

Note that, strictly speaking, [LLHLM18, Table 7] only has entries for $\widetilde{w}_{i}$ belonging to a certain set of representatives under the action of the outer automorphisms of $\widetilde{W}_{a}$.

(2) When $\ell\left(\widetilde{w}_{i}\right)=1$ : By symmetry, we may assume $\widetilde{w}_{i}=\alpha t_{\underline{1}}$. The matrix $A^{(i) \text {,univ }}$ has the form

$$
A^{(i) \text {,univ }}=\left(\begin{array}{ccc}
c_{11} & c_{12}+v c_{12}^{*} & c_{13} \\
v c_{21}^{*} & c_{22}+v d_{22} & c_{23} \\
v c_{31} & v c_{32} & c_{33}+v c_{33}^{*}
\end{array}\right) .
$$

Set $\widetilde{c}_{32} \stackrel{\text { def }}{=} \frac{c_{32} c_{21}^{*}-d_{22} c_{31}}{c_{21}^{*}}$. We define $\bar{R}_{\bar{M}^{2}, \widetilde{w}_{i}}^{\text {expl, }}$ to be the quotient of the power series ring

$$
\begin{aligned}
& \mathbb{F}\left[c_{11}, c_{12}, c_{13}, c_{22}, c_{23}, c_{31}, \widetilde{c}_{32}, c_{33}, d_{22}, c_{12}^{*}\right. \\
& \quad-\left[\bar{c}_{12}^{*}\right], c_{21}^{*}-\left[\bar{c}_{21}^{*}\right], c_{33}^{*}-\left[\bar{c}_{33}^{*}\right] \rrbracket
\end{aligned}
$$

by the following relations:

$$
c_{11} c_{23}=0, \quad c_{33}^{*} c_{11} \widetilde{c}_{32}=c_{13} c_{31} \widetilde{c}_{32},
$$




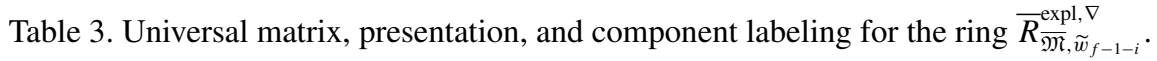

\begin{tabular}{|c|c|c|c|c|}
\hline$\widetilde{w}_{f-1-i} t_{-1}$ & $A^{(f-1-i)}$ & $\left(\omega_{i}, a_{i}\right) \in \Sigma_{\tilde{w}_{i}}$ & $c_{\left(\omega_{i}, a_{i}\right)}$ & $\bar{z}_{i}^{*}$ \\
\hline \multirow[t]{2}{*}{$\beta \alpha \gamma$} & \multirow{2}{*}{$\begin{array}{c}\left(\begin{array}{ccc}v c_{11} & v c_{12}^{*} & 0 \\
v^{2} c_{21}^{*} & v c_{22} & 0 \\
v\left(c_{31}+v d_{31}\right) & v c_{32} & c_{33}^{*}\end{array}\right) \\
c_{11} c_{22}=0 \\
(-1-a+c) c_{12}^{*} c_{31}-(-1-b+c) c_{32} c_{11}=0 \\
\end{array}$} & $\left(\varepsilon_{1}+\varepsilon_{2}, 0\right)$ & $\left(c_{11}\right)$ & $\beta \gamma^{+} \beta$ \\
\hline & & $\left(\varepsilon_{2}, 1\right)$ & $\left(c_{22}\right)$ & $\alpha$ \\
\hline \multirow[t]{2}{*}{$\alpha \beta \gamma$} & \multirow{2}{*}{$\begin{array}{c}\left(\begin{array}{ccc}v^{2} c_{11} & 0 & 0 \\
v\left(c_{21}+v d_{21}\right) & c_{22} & c_{23}^{*} \\
v\left(c_{21} c_{33}\left(c_{23}^{*}\right)^{-1}+v d_{31}\right) & v c_{32}^{*} & c_{33}\end{array}\right) \\
c_{22} c_{33}=0 ; \\
(-1-a+c) c_{21} c_{32}^{*}+(b-c) d_{31} c_{22}=0\end{array}$} & $\left(\varepsilon_{1}+\varepsilon_{2}, 0\right)$ & $\left(c_{22}\right)$ & $\alpha \gamma^{+} \alpha$ \\
\hline & & $\left(\varepsilon_{1}, 1\right)$ & $\left(c_{33}\right)$ & $\beta$ \\
\hline \multirow[t]{2}{*}{$\alpha \beta \alpha$} & \multirow{2}{*}{$\left.\begin{array}{ccc} & & \\
c_{11} & c_{11} c_{32}\left(c_{31}^{*}\right)^{-1} & d_{33} c_{11}\left(c_{31}^{*}\right)^{-1}+v c_{13}^{*} \\
0 & v c_{22}^{*} & v c_{23} \\
v c_{31}^{*} & v c_{32} & v d_{33}\end{array}\right)$} & $(0,0)$ & $\left(c_{11}\right)$ & $\gamma^{+}$ \\
\hline & & $(0,1)$ & $\left((a-b) c_{23} c_{32}-(a-c) c_{33}^{*} d_{33}\right)$ & id \\
\hline \multirow{4}{*}{$\alpha \beta$} & \multirow{4}{*}{$\left(\begin{array}{ccc}c_{13} c_{12}\left(c_{32}^{*}\right)^{-1} & c_{12} & c_{13}+v c_{13}^{*} \\
v c_{21}^{*} & c_{22} & c_{23}+v d_{23} \\
v c_{31} & v c_{32}^{*} & c_{31} c_{23}\left(c_{21}^{*}\right)^{-1}+v d_{33}\end{array}\right)$} & $\left(\varepsilon_{1}, 1\right)$ & $\left(c_{12}, c_{31}\right)$ & $\gamma^{+} \beta$ \\
\hline & & $\left(\varepsilon_{1}-\varepsilon_{2}, 0\right)$ & $\left(c_{31}, d_{33}\right)$ & $\beta \gamma^{+} \alpha \beta$ \\
\hline & & $(0,0)$ & $\left(c_{12}, c_{22}\right)$ & $\alpha \gamma^{+}$ \\
\hline & & $(0,1)$ & $\left(c_{22},(b-c) d_{33} c_{21}^{*}+(a-b) c_{31} d_{23}\right)$ & $\alpha$ \\
\hline \multirow{4}{*}{$\beta \alpha$} & \multirow{4}{*}{$\begin{array}{c}\left(\begin{array}{ccc}c_{11} & \left(c_{31}^{*}\right)^{-1} c_{11} c_{32}+v c_{12}^{*} & c_{13} \\
0 & v d_{22} & v c_{23}^{*} \\
v c_{31}^{*} & v c_{32} & c_{33}+v d_{33}\end{array}\right) \\
c_{11} c_{33}=0 ; \\
d_{22}\left(c_{13} c_{31}^{*}-c_{11} d_{33}\right)=0 ; \\
c_{11}\left((a-b) c_{32} c_{23}^{*}-(a-c) d_{22} d_{33}\right)=0 ; \\
(1+a-c) c_{33} c_{23}^{*} c_{12}^{*}=c_{13}\left((a-b) c_{32} c_{23}^{*}-(a-c) d_{22} d_{33}\right)\end{array}$} & $\left(\varepsilon_{2}, 1\right)$ & $\left(d_{22}, c_{11}\right)$ & $\gamma^{+} \alpha$ \\
\hline & & $\left(\varepsilon_{2}-\varepsilon_{1}, 0\right)$ & $\left(d_{22}, c_{32}\right)$ & $\alpha \gamma^{+} \beta \alpha$ \\
\hline & & $(0,0)$ & $\left(c_{11}, c_{13}\right)$ & $\beta \gamma^{+}$ \\
\hline & & $(0,1)$ & $\left((a-b) c_{32} c_{23}^{*}-(a-c) d_{22} d_{33}, c_{13} c_{31}^{*}-c_{11} d_{33}\right)$ & $\beta$ \\
\hline \multirow{5}{*}{$\alpha$} & \multirow{5}{*}{$\begin{array}{c}\left(\begin{array}{ccc}c_{11} & c_{12}+v c_{12}^{*} & c_{13} \\
v c_{21}^{*} & c_{22}+v d_{22} & c_{23} \\
v c_{31} & v c_{32} & \left(c_{21}^{*}\right)^{-1} c_{31} c_{23}+v c_{33}^{*}\end{array}\right) \\
\text { see } \$ 3.6 .2 \text { for the relations among coefficients }\end{array}$} & $\left(\varepsilon_{1}, 1\right)$ & $\left(c_{11}, c_{13}, c_{31}\right)$ & $\beta \gamma^{+} \beta$ \\
\hline & & $\left(\varepsilon_{2}, 0\right)$ & $\left(c_{11}, c_{31}, c_{32} c_{21}^{*}-d_{22} c_{31}\right)$ & $\gamma^{+} \alpha \beta$ \\
\hline & & $\left(\varepsilon_{2}, 1\right)$ & $\left(c_{11}, c_{32} c_{21}^{*}-d_{22} c_{31},(a-b) c_{13} d_{22}+(-1-a+c) c_{23} c_{12}^{*}\right)$ & $\gamma^{+} \alpha$ \\
\hline & & $\frac{\left(\varepsilon_{2}-\varepsilon_{1}, 0\right)}{(0,0)}$ & $\frac{\left(c_{23}, d_{22}, c_{32} c_{21}^{*}-d_{22} c_{31}\right)}{\left(c_{11}, c_{13}, c_{23}\right)}$ & $\frac{\alpha \beta \gamma^{+} \beta \alpha}{\beta \alpha \gamma^{+}}$ \\
\hline & & $(0,0)$ & $\frac{\left(c_{11}, c_{13} c_{23}\right)}{\left(c_{11} c_{33}^{*}-c_{13} c_{31}, c_{23},(a-b) c_{31} d_{22}+(c-b)\left(c_{32} c_{21}^{*}-d_{22} c_{31}\right)\right)}$ & $\beta \alpha \gamma$ \\
\hline \multirow{5}{*}{ id } & \multirow{5}{*}{$\begin{array}{l}\left(\begin{array}{ccc}c_{11}+v c_{11}^{*} & c_{12} & c_{13} \\
v c_{21} & c_{22}+v c_{22}^{*} & c_{23} \\
v c_{31} & v c_{32} & c_{33}+v c_{33}^{*}\end{array}\right) \\
\text { see } \$ 3.6 .2 \text { for the relations among coefficients }\end{array}$} & $\left(\varepsilon_{1}, 0\right)$ & $\left(c_{i i, i=1,2,3,}, c_{21}, c_{31}, c_{23}\right)$ & $\beta \beta \gamma^{+} \beta \alpha$ \\
\hline & & $\frac{\left(\varepsilon_{1}, 1\right)}{\left(\varepsilon_{2}, 0\right)}$ & $\frac{\left(c_{31}, c_{33}, c_{11},(-1-a+c) c_{32} c_{13}-(-1-a+b) c_{12} c_{33}^{*}, c_{21} c_{13}-c_{23} c_{11}^{*}\right)}{\left(c_{131}\right)}$ & $\frac{\beta \gamma^{+} \beta}{\alpha \gamma^{+} \alpha \beta}$ \\
\hline & & $\frac{\left(\varepsilon_{2}, 0\right)}{\left(\varepsilon_{2}, 1\right)}$ & $\frac{\left(c_{i i, i}, 1,2,3, c_{12}, c_{31}, c_{32}\right)}{\left(c_{12}, c_{22}, c_{11},(a-b) c_{21} c_{13}-(-1-b+c) c_{23} c_{11}^{*}, c_{21} c_{32}-c_{31} c_{22}^{*}\right)}$ & $\alpha \gamma^{+} \alpha$ \\
\hline & & $(0,0)$ & $\left(c_{i i, i=1,2,3,} c_{13}, c_{23}, c_{12}\right)$ & $\alpha \beta \alpha \gamma^{+}$ \\
\hline & & $(0,1)$ & $\left(c_{23}, c_{33}, c_{22},(a-b) c_{21} c_{32}-(a-c) c_{31} c_{22}^{*}, c_{32} c_{13}-c_{12} c_{33}^{*}\right)$ & $\alpha \beta \alpha$ \\
\hline
\end{tabular}

Data relevant to Theorem 3.6.4. The first column records the components of the shape $\widetilde{w}=\widetilde{w}(\bar{\rho}, \tau)$. The second column records the form of the matrix of partial Frobenius $A^{(f-1-i)}$ and the presentation of the ring $\bar{R}_{\mathfrak{M},}^{\operatorname{expl}_{1}, \nabla}$ in terms of the entries of $A^{(f-1-i)}$. The fourth column records the prime ideal $\mathfrak{c}_{\left(\omega_{i}, a_{i}\right)}$ in the statement of Theorem 3.6.4. The last column records the element $\widetilde{z}^{*} \in \underline{W}_{a}$ that occurs in Proposition 3.6.9, which controls the minimal type $\tau^{\prime}$ of the weight given by the third column with respect to $\bar{\rho}$. Thus, $\tau^{\prime}=\tau\left(s z^{*}, \mu+s \widetilde{z}^{*}(0)\right)$. Note that $\widetilde{w}\left(\bar{\rho}, \tau^{\prime}\right)=\widetilde{w}(\bar{\rho}, \tau) \widetilde{z}^{-1}$. Finally, the structure constants that feature in the presentation of $\bar{R}_{\overline{\mathfrak{M}}, \widetilde{w}_{f-1-i}}^{\operatorname{expl}, \nabla}$ are given by $(a, b, c) \in \mathbb{F}_{p}^{3}$ with $(a, b, c) \equiv s_{i}^{-1}\left(\mu_{i}\right) \bmod p$. 
Table 3. (continued)

\begin{tabular}{|c|c|c|c|c|}
\hline$\widetilde{w}_{f-1-i} t_{-\underline{1}}$ & $A^{(f-1-i)}$ & $\left(\omega_{i}, a_{i}\right) \in \Sigma_{\widetilde{w}_{i}^{*}}$ & $\mathfrak{w}_{\omega_{i}} \stackrel{\text { def }}{=} \mathfrak{c}_{\left(\omega_{i}, 0\right)} \cap \mathfrak{c}_{\left(\omega_{i}, 1\right)}$ & $\bar{z}_{i}^{*}$ \\
\hline$\alpha \beta$ & $\begin{array}{c}\left(\begin{array}{ccc}c_{13} c_{12}\left(c_{32}^{*}\right)^{-1} & c_{12} & c_{13}+v c_{13}^{*} \\
v c_{21}^{*} & c_{22} & c_{23}+v d_{23} \\
v c_{31} & v c_{32}^{*} & c_{31} c_{23}\left(c_{21}^{*}\right)^{-1}+v d_{33}\end{array}\right) \\
c_{12} c_{23}-c_{22} c_{13}=0 ; \\
c_{22} c_{31}=0 ; \\
c_{32}^{*} c_{13}-d_{33} c_{12}=0 ; \\
c_{12}\left((a-b) c_{31} d_{23}-(b-c) d_{33} c_{21}^{*}\right)=0 \\
(-1-a+c) c_{23} c_{32}^{*}=(-1-a+b) c_{22} d_{33}\end{array}$ & $(0,1),(0,0)$ & $\left(c_{22}\right)$ & $\alpha$ \\
\hline$\beta \alpha$ & $\begin{array}{c}\left(\begin{array}{ccc}c_{11} & \left(c_{31}^{*}\right)^{-1} c_{11} c_{32}+v c_{12}^{*} & c_{13} \\
0 & v d_{22} & v c_{23}^{*} \\
v c_{31}^{*} & v c_{32} & c_{33}+v d_{33}\end{array}\right) \\
c_{11} c_{32}=0 ; \\
d_{22}\left(c_{13} c_{31}^{*}-c_{11} d_{33}\right)=0 ; \\
c_{11}\left((a-b) c_{32} c_{23}^{*}-(a-c) d_{22} d_{33}\right)=0 ; \\
(1+a-c) c_{33} c_{23}^{*} c_{12}^{*}=c_{13}\left((a-b) c_{32} c_{23}^{*}-(a-c) d_{22} d_{33}\right)\end{array}$ & $(0,1),(0,0)$ & $\left(c_{13} c_{31}^{*}-c_{11} d_{33}\right)$ & $\beta$ \\
\hline \multirow[t]{2}{*}{$\alpha$} & \multirow{2}{*}{$\begin{array}{c}\left(\begin{array}{ccc}c_{11} & c_{12}+v c_{12}^{*} & c_{13} \\
v c_{21}^{*} & c_{22}+v d_{22} & c_{23} \\
v c_{31} & v c_{32} & \left(c_{21}^{*}\right)^{-1} c_{31} c_{23}+v c_{33}^{*}\end{array}\right) \\
\text { see } \$ 3.6 .2 \text { for the relations among coefficients }\end{array}$} & $\left(\varepsilon_{2}, 1\right),\left(\varepsilon_{2}, 0\right)$ & $\left(c_{32} c_{21}^{*}-d_{22} c_{31}, c_{11}\right)$ & $\gamma^{+} \alpha$ \\
\hline & & $(0,1),(0,0)$ & $\left(c_{11} c_{33}^{*}-c_{13} c_{31}, c_{23}\right)$ & $\beta \alpha$ \\
\hline \multirow[t]{3}{*}{ id } & \multirow{3}{*}{$\begin{array}{l}\left(\begin{array}{ccc}c_{11}+v c_{11}^{*} & c_{12} & c_{13} \\
v c_{21} & c_{22}+v c_{22}^{*} & c_{23} \\
v c_{31} & v c_{32} & c_{33}+v c_{33}^{*}\end{array}\right) \\
\text { see } \$ 3.6 .2 \text { for the relations among coefficients }\end{array}$} & $\left(\varepsilon_{1}, 1\right),\left(\varepsilon_{1}, 0\right)$ & $\left(c_{33}, c_{11}, c_{31}, c_{11}^{*} c_{23}-c_{21} c_{13}\right)$ & $\beta \gamma^{+} \beta$ \\
\hline & & $\left(\varepsilon_{2}, 1\right),\left(\varepsilon_{2}, 0\right)$ & $\left(c_{11}, c_{22}, c_{12}, c_{22}^{*} c_{31}-c_{32} c_{21}\right)$ & $\alpha \gamma^{+} \alpha$ \\
\hline & & $(0,1),(0,0)$ & $\left(c_{22}, c_{33}, c_{23}, c_{33}^{*} c_{12}-c_{13} c_{32}\right)$ & $\alpha \beta \alpha$ \\
\hline
\end{tabular}

Further data relevant to Theorem 3.6.4. The description of the columns is the same as that in Table 3.

$$
\begin{array}{rlrl}
c_{11} d_{22} c_{33}^{*} & =\frac{b-c}{a-b} c_{21}^{*} c_{13} \widetilde{c}_{32}, & c_{13} c_{23} \widetilde{c}_{32}=0, \\
c_{23} c_{31} \widetilde{c}_{32}=0, & c_{21}^{*} c_{33}=c_{31} c_{23}, \\
c_{12} c_{33}^{*}=\frac{a-c}{a-b} c_{13} \widetilde{c}_{32}, & c_{22} c_{33}^{*}=\frac{(-1-a+c)}{(-1-a+b)} c_{23} \widetilde{c}_{32},
\end{array}
$$

and

$$
(a-b) c_{13} c_{31} d_{22}+(c-b) c_{13} \widetilde{c}_{32} c_{21}^{*}+(-1-a+c) c_{23} c_{31} c_{12}^{*}=0 .
$$

These equations come from [LLHLM18, Proposition 8.11] and its proof, by restoring the units $c_{12}^{*}, c_{21}^{*}$, and $c_{33}^{*}$ (which were set to be 1 in loc. cit.); the first six equations above are deduced from the equations 
appearing in the statement of loc. cit. (where $d_{22}$ above is denoted by $c_{22}^{\prime}$ in loc. cit.), the seventh and eighth equations above appear in the proof of loc. cit., and the last equation above is implicit in loc. cit. (where we solved $c_{33}$ using the $p$-saturation of the 2 by 2 minor condition). In particular, $\bar{R}_{\mathfrak{M}, \widetilde{w}_{i}}^{\text {exl }, \nabla}$ is a formal power series ring over the ring $\widetilde{R}$ appearing in [LLHLM18, Proposition 8.11].

(3) When $\ell\left(\widetilde{w}_{i}\right)=0$, the matrix $A^{(i) \text {,univ }}$ has the form

$$
A^{(i) \text {,univ }}=\left(\begin{array}{ccc}
c_{11}+v c_{11}^{*} & c_{12} & c_{13} \\
v c_{21} & c_{22}+v c_{22}^{*} & c_{23} \\
v c_{31} & v c_{32} & c_{33}+v c_{33}^{*}
\end{array}\right) .
$$

We define $\bar{R}_{\overline{\mathfrak{M}}, \widetilde{w}_{i}}^{\text {expl, }}$ to be the quotient of $\mathbb{F} \llbracket c_{i j}, c_{k k}^{*}-\left[\bar{c}_{k k}^{*}\right], 1 \leqslant i, j$, $k \leqslant 3$ ॥ by the relations

$$
\begin{array}{lll}
c_{i i} c_{j j}=0 \quad \text { for } i \neq j, & c_{11} c_{23}=0 & c_{31} c_{22}=0, \\
c_{33} c_{12}=0, & c_{12} c_{23}=c_{22} c_{13}, & c_{11} c_{32}=c_{12} c_{31}, \\
c_{21} c_{33}=c_{31} c_{23}, & &
\end{array}
$$

and

$$
\begin{aligned}
& (-1-a+c) c_{22}^{*} c_{33}+(-1-a+b) c_{22} c_{33}^{*} \\
& \quad-(-1-a+c) c_{23} c_{32}=0, \\
& (a-b) c_{33}^{*} c_{11}+(-1-b+c) c_{33} c_{11}^{*}-(a-b) c_{13} c_{31}=0, \\
& (b-c) c_{11}^{*} c_{22}+(a-c) c_{11} c_{22}^{*}-(b-c) c_{12} c_{21}=0, \\
& c_{11} c_{22}^{*} c_{33}^{*}+c_{22} c_{11}^{*} c_{33}^{*}+c_{33} c_{11}^{*} c_{22}^{*}-c_{11}^{*} c_{23} c_{32}-c_{22}^{*} c_{13} c_{31}-c_{33}^{*} c_{12} c_{21} \\
& \quad+c_{13} c_{32} c_{21}=0 .
\end{aligned}
$$

These equations come from [LLHLM18, Corollary 8.4] by restoring the units $c_{k k}^{*}$ (which were set to be 1 in loc. cit.) and we added the equation $c_{12} c_{33}=0$, which was missing in loc. cit. and which is obtained by the 2 by 2 minor condition on $A^{(i) \text {,univ }}$. In particular, $\bar{R}_{\bar{M}, \widetilde{w}_{i}}^{\operatorname{expl}, \nabla}$ is a formal power series ring over the ring $\widetilde{R}$ appearing in [LLHLM18, Corollary 8.4].

Note that each object with a superscript $\square$ receives a $\widehat{\mathrm{GL}}_{3}$-action, corresponding to changing the framing on the Galois representation.

We now justify the diagram: 
- We claim that there is a canonical isomorphism $\left[\operatorname{Spf}\left(\bar{R}_{\overline{\mathfrak{M}}, \bar{\rho}}^{\tau, \bar{\beta}, \square}\right) / \widehat{\mathrm{GL}}_{3}\right] \cong$ $\operatorname{Spf}\left(\bar{R}_{\overline{\mathfrak{M}}, \widetilde{w}}^{\exp , \nabla}\right)$. When $\ell\left(\widetilde{w}_{i}\right)>1$ for all $i$, this is [LLHLM18, Theorem 5.12, Theorem 6.14 and Table 7]. When $\ell\left(\widetilde{w}_{i}\right) \leqslant 1$ for some $i$, this follows from the same arguments as [LLHLM18, Section 8] together with Theorem 3.5.2, as explained in the proof of Theorem 3.5.3. This justifies the existence and the properties of the first row of Diagram (3.9). It is clear from the construction that the $\widehat{\mathbf{G}}_{m}^{3 f}$-action on $\bar{D} \overline{\mathfrak{M}}_{\bar{\beta}}^{\tau, \bar{\beta}}$ preserves $\operatorname{Spf}\left(\bar{R}_{\overline{\mathfrak{M}}, \widetilde{w}}^{\text {expl, }}\right)$. Let $\left(\mathfrak{M}_{\widetilde{w}, \tau}, \beta_{\widetilde{w}, \tau}\right)$ be the restriction of $\left(\mathfrak{M}^{\text {univ }}, \beta^{\text {univ }}\right)$ to $\operatorname{Spf}\left(\overline{R_{\bar{M}}^{\text {expl, },}}\right)$. Thus, the map $\iota_{\tau}$ sends $\mathfrak{M}_{\widetilde{w}, \tau}$ to $\mathcal{M}_{\widetilde{w}, \tau} \stackrel{\text { def }}{=}\left(\mathfrak{M}_{\widetilde{w}, \tau}\left[\frac{1}{u^{\prime}}\right]\right)^{\Delta=1}$.

- The second row is obtained from the first row by quotienting by the $\widehat{\mathbf{G}}_{m}^{3 f}$-action coming from scaling the gauge basis and, hence, inherits all properties from the first row. The top squares are Cartesian.

- The second column is obtained from the first column by quotienting by the $\widehat{\mathrm{GL}}_{3}$-action coming from changing the framing of the Galois representation; hence, the bottom square is Cartesian.

The following proposition finishes our justification of the diagram.

Proposition 3.6.3. Assume that $\tau$ is 3-generic and that $\overline{\mathfrak{M}} \in \bar{Y}^{\eta, \tau}(\mathbb{F})$ is semisimple of shape $\widetilde{w}=\left(\widetilde{w}_{j}\right)$. Let $\bar{\beta}$ be a gauge basis for $\overline{\mathfrak{M}}$. Then the map

$$
l_{\tau}^{\prime}: \bar{Y}_{\bar{M}}^{\eta, \tau} \rightarrow \Phi-\operatorname{Mod}_{\mathcal{M}}^{e t}
$$

is a monomorphism.

Proof. We need to prove that the map on the groupoids of $\mathbb{F}[\varepsilon] /\left(\varepsilon^{2}\right)$-points

$$
\bar{Y} \overline{\bar{M}^{\prime} \tau}\left(\mathbb{F}[\varepsilon] /\left(\varepsilon^{2}\right)\right) \rightarrow \Phi-\operatorname{Mod}_{\mathcal{M}}\left(\mathbb{F}[\varepsilon] /\left(\varepsilon^{2}\right)\right)
$$

induced by $l_{\tau}^{\prime}$ is fully faithful. But this follows from [LLHL19, Proposition 3.2.18], noting that the right-hand side is equivalent to $\operatorname{Rep}_{\mathbb{F}[\varepsilon] / \varepsilon^{2}}\left(G_{K_{\infty}}\right)_{\bar{\rho}}$ in loc. cit.

Diagram (3.9) gives a bijection between the set of minimal primes $\operatorname{Irr}\left(\operatorname{Spec}\left(\bar{R}_{\bar{\rho}}^{\tau}\right)\right)$ and $\operatorname{Irr}\left(\operatorname{Spec}\left(\bar{R}_{\overline{\mathfrak{M}}, \widetilde{w}}^{\exp , \nabla}\right)\right)=\prod_{i} \operatorname{Irr}\left(\operatorname{Spec}\left(\bar{R}_{\overline{\mathfrak{M}}^{\mathrm{w}}, \widetilde{w}_{i}}^{\text {expl, }}\right)\right)$. By Proposition 3.6.1, this set is in bijection with the set $\mathfrak{p}(\sigma)$ for $\sigma \in W^{?}(\bar{\rho}, \tau)$. On the other hand, by Proposition 3.4.2, we have a bijection

$$
\prod_{i} \Sigma_{\widetilde{w}_{i}^{*}} \stackrel{\sim}{\rightarrow} W^{?}(\bar{\rho}, \tau)
$$




$$
(\omega, a) \mapsto \sigma_{(\omega, a)}^{(s, \mu)} .
$$

The following theorem is the main result of this subsection, which computes the above bijections in terms of the explicit rings.

THEOREM 3.6.4. Assume $\bar{\rho}$ is 10-generic. Via the above bijections, we have

$$
\begin{gathered}
\prod_{i=0}^{f-1} \Sigma_{\widetilde{w}_{i}^{*}} \stackrel{\sim}{\longrightarrow} \prod_{i} \operatorname{Irr}\left(\operatorname{Spec}\left(\bar{R}_{\overline{\mathfrak{M}}, \widetilde{w}_{i}}^{\exp , \nabla}\right)\right) \stackrel{\sim}{\longrightarrow} \operatorname{Irr}\left(\operatorname{Spec}\left(\bar{R}_{\bar{\rho}}^{\tau}\right)\right) \\
\left(\left(\omega_{i}, a_{i}\right)\right)_{i} \longmapsto\left(\left(\mathfrak{c}_{\left(\omega_{f-1-i}, a_{f-1-i}\right)} \bar{R}_{\mathfrak{M}, \widetilde{w}_{i}}^{\operatorname{expl}, \nabla}\right)_{i} \longmapsto \mathfrak{p}\left(\sigma_{(\omega, a)}^{(s, \mu)}\right),\right.
\end{gathered}
$$

where $\mathfrak{c}_{\left(\omega_{i}, a_{i}\right)}$ is the minimal prime of $\bar{R}_{\mathfrak{M}, \widetilde{w}_{f-1-i}}^{\operatorname{expl}, \nabla}$ given by Table 3.

REMARK 3.6.5. Note that Table 3 only gives the ideals $\mathfrak{c}_{\left(\omega_{i}, a_{i}\right)} \subseteq \bar{R}_{\overline{\mathfrak{M}}_{,}, \widetilde{w}_{f-1-i}}^{\operatorname{exl}, \nabla}$ for a set of representatives for $\widetilde{w}_{f-1-i} \in \operatorname{Adm}^{\vee}((2,1,0))$ for the action of the outer automorphisms of $\widetilde{W}^{\vee}$. This is sufficient because this action corresponds to changing the lowest alcove presentation of $\tau$.

We now describe the strategy of proof. The main idea is that by the proof of Proposition 3.6.1, the prime $\mathfrak{p}(\sigma)$ can be characterized by the relation (3.6) for a specific type $\tau^{\prime}$ (inducting on defect of $\sigma$ ). Namely, one can use the minimal type $\tau^{\prime}$ of $\sigma$ with respect to $\bar{\rho}$. For this type, each component of $\widetilde{w}^{\prime}=\widetilde{w}\left(\bar{\rho}, \tau^{\prime}\right)$ has length $\geqslant 3$. Furthermore, the minimal type $\tau^{\prime}$ has the property that $\operatorname{Spf} \bar{R}_{\bar{\rho}}^{\tau^{\prime}} \subset \operatorname{Spf} \bar{R}_{\bar{\rho}}^{\tau}$ inside $\operatorname{Spf} \bar{R}_{\bar{\rho}}^{\square}$. Thus, it suffices to determine the subset of $\operatorname{Irr}\left(\operatorname{Spec}\left(\bar{R}_{\overline{\mathfrak{M}}, \widetilde{w}_{i}}^{\operatorname{expl}, \nabla}\right)\right)$ which occurs in $\bar{R}_{\bar{\rho}}^{\tau^{\prime}}$. This is achieved by 'matching' the universal étale $\varphi$-module over a union of irreducible components of $\bar{R}_{\overline{\mathfrak{M}}, \widetilde{w}}^{\exp , \nabla}$ with the universal étale $\varphi$-module living over $\bar{R}_{\bar{\rho}}^{\tau^{\prime}}$ (or rather $\bar{R}_{\overline{\mathfrak{M}}^{\prime}, \widetilde{w}^{\prime}}^{\exp , \nabla}$.

The precise formulation of this matching mechanism is given by the following.

LEMMA 3.6.6. Let $\tau=\tau(s, \mu)$ and $\tau^{\prime}=\tau\left(s^{\prime}, \mu^{\prime}\right)$. Assume that $R_{\bar{\rho}}^{\tau}, R_{\bar{\rho}}^{\tau^{\prime}} \neq 0$, and the running hypothesis that $\bar{\rho}$ is 10-generic. Consider diagram (3.9) for $R_{\bar{\rho}}^{\tau}, R_{\bar{\rho}}^{\tau^{\prime}}$, constructed using the above presentations. We decorate the objects that occur in the diagram for $\tau^{\prime}$ with the same symbol as those in the diagram for $\tau$ but with a superscript ' added (so we have, for example, $\widetilde{w}^{\prime}, \mathfrak{M}_{\widetilde{w}^{\prime}, \tau^{\prime}}$, and so on). Assume

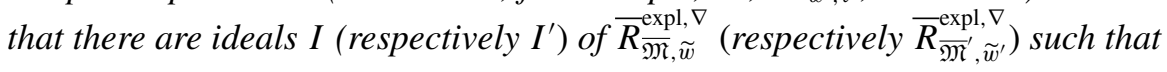

- $I, I^{\prime}$ are intersections of minimal primes.

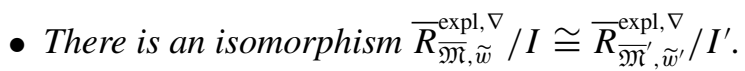


- There exists an isomorphism of étale $\varphi$-modules between the base change of $\mathcal{M}_{\widetilde{w}, \tau}$ to $\bar{R}_{\overline{\mathfrak{M}}, \widetilde{w}}^{\operatorname{expl}, \nabla} / I$ and the base change of $\mathcal{M}_{\widetilde{w}^{\prime}, \tau^{\prime}}$ to $\bar{R}_{\overline{\mathfrak{M}}^{\prime}, \widetilde{w}^{\prime}}^{\text {expl, }} / I^{\prime}$ compatible with the above ring homomorphism.

Let $J$ (respectively $J^{\prime}$ ) denote the intersection of minimal primes in $\bar{R}_{\bar{\rho}}^{\tau}$ (respectively $\bar{R}_{\bar{\rho}}^{\tau^{\prime}}$ ) which correspond to $I$ (respectively $I^{\prime}$ ). Then, $J$ and $J^{\prime}$ induce the same ideals in $\bar{R}_{\bar{\rho}}^{\square}$.

Proof. Our hypotheses imply that there is an isomorphism

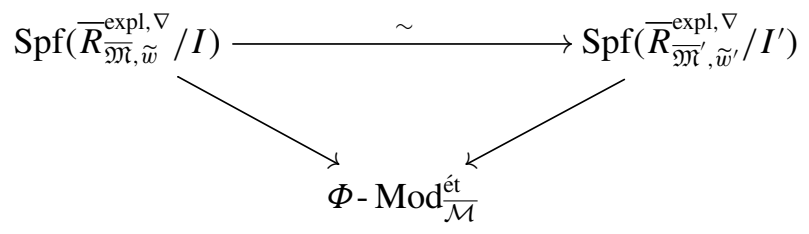

Pulling this back along the map $\Phi$ - $\operatorname{Mod}_{\mathcal{M}}^{\text {ét, } \square} \rightarrow \Phi$ - $\operatorname{Mod}_{\overline{\mathcal{M}}}^{\text {ét }}$ gives a commutative diagram

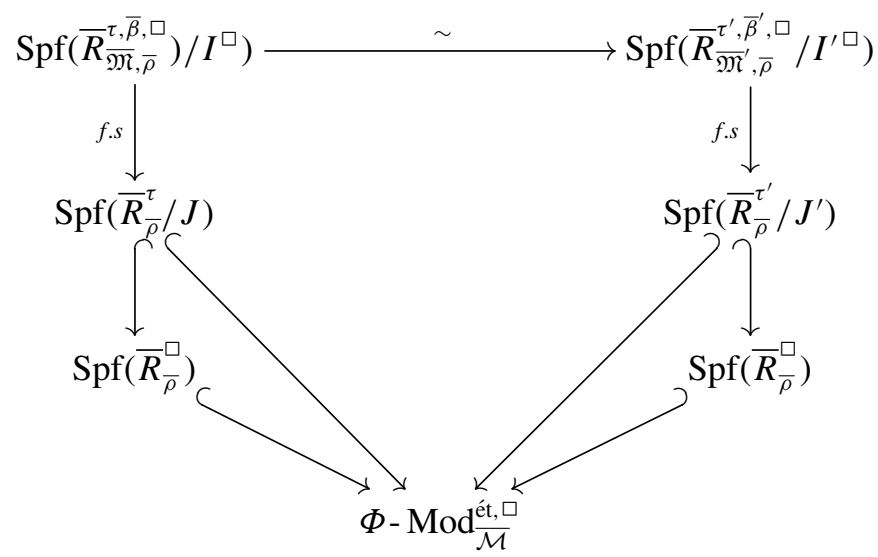

where we use [LLHLM18, Proposition 3.12] to see that the natural map $\operatorname{Spf}\left(\bar{R}_{\bar{\rho}}^{\square}\right) \rightarrow \Phi-\operatorname{Mod}_{\overline{\mathcal{M}}}^{\text {ét, }}$ is a monomorphism (as $\bar{\rho} 10$-generic implies $\operatorname{ad}(\bar{\rho})$ is cyclotomic free). But this implies that $\operatorname{Spf}\left(\bar{R}_{\bar{\rho}}^{\tau} / J\right)$ and $\operatorname{Spf}\left(\bar{R}_{\bar{\rho}}^{\tau^{\prime}} / J^{\prime}\right)$ define the same subfunctor of $\Phi-\operatorname{Mod}_{\overline{\mathcal{M}}}$ é, $\square$ and, hence, also the same subfunctor of $\operatorname{Spf}\left(\bar{R}_{\bar{\rho}}^{\square}\right)$.

In practice, we will apply the lemma by matching matrices of partial Frobenii. 
COROLlary 3.6.7. Keep the notations and setting of Lemma 3.6.6. Assume that there exists $\widetilde{z}=\left(\widetilde{z}_{i}\right)_{i} \in \underline{W}_{a}^{\vee}$ with corresponding $\widetilde{z}^{*}=\left(\widetilde{z}_{i}^{*}\right)_{i} \in \underline{W}_{a}$ and $z$ is the image of $\tilde{z}$ in $W^{\vee}$ such that $s^{\prime}=s z^{*}$ and $\mu^{\prime}=\mu+s \widetilde{z}^{*}(0)$. Write $\left(I_{i}\right)_{i}$ for the collection of ideals in $\overline{R_{\bar{M}}} \widetilde{w}_{i}$ giving rise to $I$, write $A^{(i)}$ for the matrix of the ith partial Frobenius of $\mathfrak{M}_{\widetilde{w}, \tau}$ with respect to $\beta_{\widetilde{w}, \tau}$, and let $\left(I_{i}^{\prime}\right)_{i}$ and $A^{\prime(i)}$ be the analogous objects for $\tau^{\prime}$. Assume the following:

- The isomorphism $\bar{R}_{\bar{M}, \widetilde{w}}^{\text {expl, }} / I \cong \bar{R}_{\overline{\mathfrak{M}}^{\prime}, \widetilde{w}^{\prime}}^{\text {expl, }} / I^{\prime}$ is induced by a collection of isomorphisms $\bar{R}_{\overline{\mathfrak{M}}, \widetilde{w}_{i}}^{\operatorname{expl}, \nabla} / I_{i} \cong \bar{R}_{\overline{\mathfrak{M}}^{\prime}, \widetilde{w}_{i}^{\prime}}^{\text {expl, }} / I_{i}^{\prime}$.

- For each $i$,

$$
A^{(f-1-i)} \bmod I_{f-1-i}=A^{\prime(f-1-i)} \widetilde{z}_{f-1-i} \bmod I_{f-1-i}^{\prime}
$$

via the above isomorphisms.

Then the same conclusion as Lemma 3.6.6 holds.

Proof. This follows from Lemma 3.6.6, Proposition 3.2.1, and the fact that $\widetilde{z} s^{*} t_{\mu^{*}}=s^{\prime *} t_{\mu^{*}}$.

REMARK 3.6.8. Note that the second condition in Corollary 3.6.7 implies in particular that

$$
\left(\bar{A}^{(i)}\right)_{i}=\left(\bar{A}^{\prime(i)} \widetilde{z}_{i}\right)_{i},
$$

where $\bar{A}^{(i)}, \bar{A}^{\prime(i)}$ denote the reductions modulo the maximal ideal. We now explain how, in the situations where we apply Corollary 3.6.7, we can always arrange this.

If we have $\bar{\rho}$ semisimple and $\tau, \tau^{\prime}, \widetilde{z}$ as in Proposition 3.6.9, then

$$
\widetilde{w}=\widetilde{w}^{\prime} \widetilde{z}
$$

Furthermore, for any choices of gauge bases $\beta, \beta^{\prime}$, for $\mathfrak{M}_{\widetilde{w}, \tau}$ and $\mathfrak{M}_{\widetilde{w}^{\prime}, \tau^{\prime}}$, respectively, one has $\bar{A}^{(i)} \in T(\mathbb{F}) \widetilde{w}_{i}$ and $\bar{A}^{\prime(i)} \in T(\mathbb{F}) \widetilde{w}_{i}^{\prime}$ by Proposition 3.3.6. Let $\mathfrak{M}_{\widetilde{w}, \tau}^{\prime}$ denote the element of $Y^{\eta, \tau}(\mathbb{F})$ with eigenbasis $\beta_{\mathfrak{M}^{\prime}}$ such that the partial Frobenii is given by $\left(\bar{A}^{\prime(i)} \widetilde{z}_{i}\right)_{i}$. (Note that $\mathfrak{M}_{\tilde{w}, \tau}^{\prime} \in Y^{\eta, \tau}(\mathbb{F})$ because it has shape $\widetilde{w} \in \operatorname{Adm}^{\vee}(\eta)$.) Since $\widetilde{z} s^{*} t_{\mu^{*}}=s^{\prime *} t_{\mu^{* *}},\left(\mathfrak{M}_{\widetilde{w}, \tau}^{\prime}\right)^{\Delta=1} \cong\left(\mathfrak{M}_{\widetilde{w}^{\prime}, \tau^{\prime}}\right)^{\Delta=1}$ by Proposition 3.2.1. Thus, $T_{\mathrm{dd}}^{*}\left(\mathfrak{M}_{\widetilde{w}, \tau}^{\prime}\right) \cong \bar{\rho}$. By the triviality of Kisin variety (see [LLHLM18, Theorem 3.2]), $\mathfrak{M}_{\widetilde{w}, \tau}^{\prime} \cong \mathfrak{M}_{\widetilde{w}, \tau}$ and $\beta_{\mathfrak{M}^{\prime}}$ defines a gauge basis of $\mathfrak{M}_{\widetilde{w}, \tau}$. Thus, by replacing $\beta_{\mathfrak{M}}$ by $\beta_{\mathfrak{M}^{\prime}}$ (this can be done by scaling by an element of $\underline{T}(\mathbb{F})$ by [LLHL19, Proposition 3.2.22]), we can then ensure that condition (3.12) holds. In all the matching that we perform below, we will always assume that the gauge bases have been chosen so that condition (3.12) holds. 
The following proposition describes all the types $\tau^{\prime}$ for which we need to perform some matching with $\tau$.

Proposition 3.6.9. Assume that $\bar{\rho}$ is 10-generic. Let $\tau=\tau(s, \mu)$ be a tame inertial type such that $R_{\bar{\rho}}^{\tau} \neq 0$, and let $\widetilde{w}=\widetilde{w}(\bar{\rho}, \tau)=\left(\widetilde{w}_{i}\right)_{i}$. Let $(\omega, a)=\left(\left(\omega_{i}\right.\right.$, $\left.\left.a_{i}\right)\right)_{i} \in \prod_{i} \Sigma_{\widetilde{w}_{i}^{*}}$ and $\sigma=\sigma_{(\omega, a)}^{(s, \mu)} \in W^{?}(\bar{\rho}, \tau)$. Let $\tau^{\prime}$ be the minimal type of $\sigma$ with respect to $\bar{\rho}$; $c f$. Remark 3.5.10. Then we have the following:

- For $\widetilde{z}^{*}=\left(\widetilde{z}_{i}^{*}\right)_{i} \in \underline{\widetilde{W}}_{a}$ as given by Table 3, we have $\tau^{\prime}=\tau\left(s^{\prime}, \mu^{\prime}\right)$ where

$$
s^{\prime}=s z^{*}, \quad \mu^{\prime}=\mu+s \widetilde{z}^{*}(0)
$$

- $\widetilde{w}\left(\bar{\rho}, \tau^{\prime}\right) \widetilde{z}=\widetilde{w} ;$ and

- $W^{?}\left(\bar{\rho}, \tau^{\prime}\right) \subset W^{?}(\bar{\rho}, \tau)$.

Proof. This is immediate by computing the pairwise intersections among $\Sigma_{0}$, $\widetilde{w}_{i}^{*}\left(r\left(\Sigma_{0}\right)\right)$, and $\widetilde{z}_{i}^{*}\left(\Sigma_{0}\right)$.

Proof of Theorem 3.6.4. By Proposition 3.6.9 and the proof of Proposition 3.6.1, for each $\sigma \in W^{?}(\bar{\rho}, \tau)$ with minimal type $\tau^{\prime}$ with respect to $\bar{\rho}$, we need to show that the intersection of minimal primes

$$
\bigcap_{(\omega, a) \in \Sigma \cap \widetilde{z}^{*}(\Sigma)}\left(\sum_{i=0}^{f-1} \mathfrak{c}_{\left(\omega_{i}, a_{i}\right)} \bar{R}_{\overline{\mathfrak{M}}, \widetilde{w}}^{\text {expl, }}\right)=\sum_{i=0}^{f-1}\left(\bigcap_{\left(\omega_{i}, a_{i}\right) \in \Sigma_{0} \cap \widetilde{Z}_{i}^{*}\left(\Sigma_{0}\right)} \mathfrak{c}_{\left(\omega_{i}, a_{i}\right)} \bar{R}_{\overline{\mathfrak{M}}, \widetilde{w}}^{\text {expl, }}\right)
$$

of $\bar{R}_{\overline{\mathfrak{M}}, \widetilde{w}}^{\exp , \nabla}$ corresponds to the intersection of primes $\bigcap_{\sigma^{\prime} \in W^{?}\left(\bar{\rho}, \tau^{\prime}\right)} \mathfrak{p}\left(\sigma^{\prime}\right)$ of $\bar{R}_{\bar{\rho}}^{\square}$. But this follows from Corollary 3.6.7 (which allows us to work for each $i$ separately) and the explicit computations in Section 3.6.2.

We record the following lemma, which follows from Theorem 3.6.4, for future use. Let $\widetilde{w}=\widetilde{w}(\bar{\rho}, \tau)$. Suppose that $\ell\left(\widetilde{w}_{i}\right) \geqslant 2$ for all $0 \leqslant i \leqslant f-1$. Let $N \stackrel{\text { def }}{=} \sum_{i}\left(4-\ell\left(\widetilde{w}_{i}\right)\right)$, and $R_{N} \stackrel{\text { def }}{=} \widehat{\bigotimes}_{j=1}^{N} \mathcal{O} \llbracket x_{j}, y_{j} \rrbracket /\left(x_{j} y_{j}-p\right)$. In this case, there exists a formally smooth map $R_{N} \rightarrow R_{\overline{\mathfrak{M}}, \widetilde{w}}^{\text {expl, }}$, which we fix. We think of $R_{N}$ as a subalgebra of $R_{\overline{\mathfrak{M}}, \widetilde{w}}^{\operatorname{expl}, \nabla}$. For $\sigma \in W^{?}(\bar{\rho}, \tau)$, let $\mathfrak{p}^{\text {expl }}(\sigma) \subset R_{\overline{\mathfrak{M}}, \widetilde{w}}^{\text {expl, }}$ be the prime ideal corresponding to the weight $\sigma$. The minimal prime ideals of $R_{\overline{\mathfrak{M}}, \widetilde{w}}^{\text {exp }, \nabla}$ containing $\varpi$ are of the form $\left(\left(z_{j}\right)_{j=1}^{N}+(\varpi)\right) R_{\overline{\mathfrak{M}}, \widetilde{w}}^{\text {expl, }}$ where $z_{j} \in\left\{x_{j}, y_{j}\right\}$ for all $1 \leqslant j \leqslant N$. Define $z_{j}(\sigma)$ so that $\mathfrak{p}^{\text {expl }}(\sigma)=\left(\left(z_{j}(\sigma)\right)_{j=1}^{N}+(\varpi)\right) R_{\bar{M}}^{\operatorname{expl}, \widetilde{w}}$. 
LeMma 3.6.10. Assume that $\bar{\rho}$ is 10 -generic and that $\ell\left(\widetilde{w}_{i}\right) \geqslant 2$ for all $0 \leqslant i \leqslant$ $f-1$. For $\sigma_{1}$ and $\sigma_{2} \in W^{?}(\bar{\rho}, \tau)$,

$$
\#\left(\left\{z_{j}\left(\sigma_{1}\right)\right\}_{j=1}^{N} \Delta\left\{z_{j}\left(\sigma_{2}\right)\right\}_{j=1}^{N}\right)=2 \mathrm{~d}_{\mathrm{gph}}\left(\sigma_{1}, \sigma_{2}\right),
$$

where $\Delta$ denotes the symmetric difference.

Proof. From the $\alpha \beta \alpha, \alpha \beta$, and $\beta \alpha$ rows of Table 3, we check that if $\sigma_{1}, \sigma_{2} \in$ $W^{?}(\bar{\rho}, \tau)$, then $\mathfrak{p}^{\operatorname{expl}}\left(\sigma_{1}\right)+\mathfrak{p}^{\operatorname{expl}}\left(\sigma_{2}\right)$ is a prime ideal of $R_{\overline{\mathfrak{M}}, \widetilde{w}}^{\operatorname{expl}, \nabla}$ of height

$$
\mathrm{d}_{\mathrm{gph}}\left(\sigma_{1}, \sigma_{2}\right)+1
$$

On the other hand, the height of the intersection of $\left(\left(z_{j}\right)_{j}+(\varpi)\right)+\left(\left(z_{j}^{\prime}\right)_{j}+(\varpi)\right)$ is easily seen to be

$$
\frac{1}{2} \#\left(\left\{z_{j}\right\}_{j=1}^{N} \Delta\left\{z_{j}^{\prime}\right\}_{j=1}^{N}\right)+1 .
$$

For convenience, we list in Table 4 the length 4 shapes and their universal families over $\bar{R}_{\overline{\mathfrak{M}}_{,} \widetilde{w}_{j}}^{\text {expl, }}$.

3.6.2. Explicit computations. In this section, we record the matching of matrices of partial Frobenii needed in the proof of Theorem 3.6.4. We are always in the setting of the theorem. In particular, we have two types $\tau, \tau^{\prime}$ with chosen presentations related by the element $\widetilde{z}^{*}$ together with matrices of partial Frobenii $A^{(f-1-i)}, A^{\prime(f-1-i)}$. We will fix $i$ throughout.

We will frequently recall presentations of rings from [LLHLM18], and since we work in characteristic $p$, all occurrences of the symbol $e$ in loc. cit. will become -1 here. We let $(a, b, c)$ and $\left(a^{\prime}, b^{\prime}, c^{\prime}\right) \in \mathbb{F}_{p}^{3}$ be such that

$$
(a, b, c)=s_{i}^{-1}\left(\mu_{i}\right) \quad \bmod p, \quad\left(a^{\prime}, b^{\prime}, c^{\prime}\right)=\left(s_{i}^{\prime}\right)^{-1}\left(\mu_{i}^{\prime}\right) \quad \bmod p .
$$

Note that $\left(a^{\prime}, b^{\prime}, c^{\prime}\right)=\widetilde{z}_{f-1-i}(a, b, c)$. These are the structure constants that feature in the presentation of our explicit rings. We will also replace occurrences of the symbols $c_{i j}^{\prime}$ in loc. cit. by $d_{i j}$ as we wish to decorate objects associated with $\tau^{\prime}$ with a prime superscript.

Case $\widetilde{w}_{f-1-i}=\alpha \beta t_{\underline{1}}$. From [LLHLM18, Table 5], the matrix of the partial Frobenius $A^{(f-1-i)}$ has the form

$$
\left(\begin{array}{ccc}
c_{31} c_{12}\left(c_{32}^{*}\right)^{-1} & c_{12} & c_{13}+v c_{13}^{*} \\
v c_{21}^{*} & c_{22} & c_{23}+v d_{23} \\
v c_{31} & v c_{32}^{*} & \left(c_{31} c_{23}\left(c_{21}^{*}\right)^{-1}+v d_{33}\right)
\end{array}\right)
$$


Table 4. Universal matrix and presentation for the rings $\bar{R}_{\overline{\mathfrak{M}}, \widetilde{w}_{f-1-i}}^{\operatorname{expl}, \nabla}$ for extremal and shadow shapes.

\begin{tabular}{|c|c|c|c|}
\hline$\widetilde{w}_{f-1-i}^{\prime} t_{-1}$ & $A^{\prime(f-1-i)}$ & $\widetilde{w}_{f-1-i}^{\prime} t_{-\underline{1}}$ & $A^{\prime(f-1-i)}$ \\
\hline$\alpha \beta \alpha \gamma$ & $\left(\begin{array}{ccc}v^{2} c_{11}^{\prime *} & 0 & 0 \\
v^{2} c_{21}^{\prime} & v c_{22}^{\prime *} & 0 \\
v^{2} d_{31}^{\prime} & v c_{32}^{\prime} & c_{33}^{\prime *}\end{array}\right)$ & $\beta \gamma \alpha \gamma$ & $\begin{array}{c}\left(\begin{array}{ccc}v c_{11}^{\prime *} & v c_{12}^{\prime} & 0 \\
0 & v^{2} c_{22}^{\prime *} & 0 \\
v c_{31}^{\prime} & v c_{32}^{\prime}+v^{2} d_{32}^{\prime} & c_{33}^{\prime *}\end{array}\right) \\
\left(-1-b^{\prime}+c^{\prime}\right) c_{32}^{\prime} c_{11}^{\prime *}-\left(-1-a^{\prime}+c^{\prime}\right) c_{12}^{\prime} c_{31}^{\prime}=0\end{array}$ \\
\hline$\beta \gamma \beta \alpha$ & $\left(\begin{array}{ccc}c_{11}^{\prime *} & v d_{12}^{\prime} & c_{13}^{\prime} \\
0 & v^{2} c_{22}^{\prime *} & 0 \\
0 & v^{2} c_{32}^{\prime} & v c_{33}^{\prime *}\end{array}\right)$ & $\gamma \alpha \beta \alpha$ & $\begin{array}{c}\left(\begin{array}{ccc}c_{11}^{\prime *} & c_{12}^{\prime} & c_{13}^{\prime}+v d_{13}^{\prime} \\
0 & v c_{22}^{\prime *} & v c_{23}^{\prime} \\
0 & 0 & v^{2} c_{33}^{\prime *}\end{array}\right) \\
\left(a^{\prime}-c^{\prime}\right) c_{13}^{\prime} c_{22}^{\prime *}-\left(a^{\prime}-b^{\prime}\right) c_{23}^{\prime} c_{12}^{\prime}=0\end{array}$ \\
\hline$\alpha \gamma \alpha \beta$ & $\left(\begin{array}{ccc}v c_{11}^{\prime *} & 0 & v c_{13}^{\prime} \\
v c_{12}^{\prime} & c_{22}^{\prime *} & v d_{23}^{\prime} \\
0 & 0 & v^{2} c_{33}^{\prime \prime}\end{array}\right)$ & $\alpha \beta \gamma \beta$ & $\begin{array}{c}\left(\begin{array}{ccc}v^{2} c_{11}^{\prime *} & 0 & 0 \\
v\left(c_{21}^{\prime}+v d_{21}^{\prime}\right) & c_{22}^{\prime *} & c_{23}^{\prime} \\
v^{2} c_{31}^{\prime} & 0 & v c_{33}^{\prime *}\end{array}\right) \\
\left(-1-a^{\prime}+b^{\prime}\right) c_{21}^{\prime} c_{33}^{\prime *}-\left(b^{\prime}-c^{\prime}\right) c_{31}^{\prime} c_{23}^{\prime}=0\end{array}$ \\
\hline$\alpha \beta \alpha$ & $\begin{array}{c}\left(\begin{array}{ccc}c_{11}^{\prime} & c_{11}^{\prime} c_{32}^{\prime}\left(c_{31}^{\prime *}\right)^{-1} & c_{11}^{\prime} d_{33}^{\prime}\left(c_{31}^{\prime *}\right)^{-1}+v c_{13}^{\prime *} \\
0 & v c_{22}^{\prime *} & v c_{23}^{\prime} \\
v c_{31}^{\prime *} & v c_{32}^{\prime} & v d_{33}^{\prime}\end{array}\right) \\
c_{11}^{\prime}\left(\left(a^{\prime}-b^{\prime}\right) c_{23}^{\prime} c_{32}^{\prime}-\left(a^{\prime}-c^{\prime}\right) c_{22}^{\prime *} d_{33}^{\prime}\right)=0\end{array}$ & $\beta \gamma \beta$ & $\begin{array}{c}\left(\begin{array}{ccc}v d_{11}^{\prime} & c_{12}^{\prime *} & c_{13}^{\prime} \\
v\left(c_{22}^{\prime} d_{11}^{\prime}\left(c_{12}^{\prime *}\right)^{-1}+v c_{21}^{\prime *}\right) & c_{22}^{\prime} & c_{22}^{\prime} c_{13}^{\prime}\left(c_{12}^{\prime \prime}\right)^{-1} \\
v^{2} c_{31}^{\prime} & 0 & v c_{33}^{\prime *}\end{array}\right) \\
c_{22}^{\prime}\left(\left(b^{\prime}-c^{\prime}\right) c_{31}^{\prime} c_{13}^{\prime}-\left(-1-a^{\prime}+b^{\prime}\right) c_{33}^{\prime *} d_{11}^{\prime}\right)=0\end{array}$ \\
\hline$\gamma \alpha \gamma$ & $\begin{array}{l}\left(\begin{array}{ccc}v c_{11}^{\prime *} & v c_{12}^{\prime} & 0 \\
v c_{21}^{\prime} & v d_{22}^{\prime} & c_{23}^{\prime *} \\
v c_{33}^{\prime} c_{21}^{\prime}\left(c_{23}^{\prime *}\right)^{-1} & v\left(c_{33}^{\prime} d_{22}^{\prime}\left(c_{23}^{\prime *}\right)^{-1}+v c_{32}^{\prime *}\right) & c_{33}^{\prime}\end{array}\right) \\
c_{33}^{\prime}\left(\left(-1-a^{\prime}+c^{\prime}\right) c_{12}^{\prime} c_{21}^{\prime}-\left(1-b^{\prime}+c^{\prime}\right) c_{11}^{\prime *} d_{22}^{\prime}\right)=0\end{array}$ & & \\
\hline
\end{tabular}

The relevant data for the type $\tau^{\prime}$ occurring in the proof of Theorem 3.6.4. The first column records the components of the shape $\widetilde{w}^{\prime}=\widetilde{w}\left(\bar{\rho}, \tau^{\prime}\right)$. The second column records the form of the matrix of partial Frobenius $A^{\prime(f-1-i)}$ and the presentation of the ring $\bar{R}_{\overline{\mathfrak{M}}^{\prime}, \widetilde{w}_{f-1-i}^{\prime}}^{\operatorname{expl}, \nabla}$ in terms of the entries of $A^{\prime(f-1-i)}$. The structure constants that feature in the presentation of $\bar{R}_{\overline{\mathfrak{M}}^{\prime}, \widetilde{w}_{f-1-i}^{\prime}}^{\operatorname{expl}, \nabla}$ are given by $\left(a^{\prime}, b^{\prime}, c^{\prime}\right) \in \mathbb{F}_{p}^{3}$ where $\left(a^{\prime}, b^{\prime}\right.$, $\left.c^{\prime}\right) \equiv\left(s_{i}^{\prime}\right)^{-1}\left(\mu_{i}^{\prime}\right) \bmod p$. Note that $\left(s_{i}^{\prime}\right)^{-1}\left(\mu_{i}^{\prime}\right) \equiv \widetilde{z}_{f-1-i}(a, b, c) \bmod p$.

The ring $\bar{R}_{\mathfrak{M}, \widetilde{w}_{f-1-i}}^{\operatorname{expl}, \nabla}$ is the quotient of $\mathbb{F} \llbracket c_{12}, c_{13}, c_{22}, c_{23}, d_{23}, c_{31}, d_{33}, c_{13}^{*}-\left[\bar{c}_{13}^{*}\right]$, $c_{21}^{*}-\left[\bar{c}_{21}^{*}\right], c_{32}^{*}-\left[\bar{c}_{32}^{*}\right] \rrbracket$ by the relations

$$
\begin{aligned}
c_{12} c_{23}-c_{22} c_{13} & =0 \\
c_{22} c_{31} & =0
\end{aligned}
$$




$$
\begin{aligned}
c_{32}^{*} c_{13}-d_{33} c_{12} & =0 ; \\
c_{12}\left((b-c) d_{33} c_{21}^{*}+(a-b) c_{31} d_{23}\right) & =0 ; \\
(-1-a+c) c_{23} c_{32}^{*} & =(-1-a+b) c_{22} d_{33} .
\end{aligned}
$$

Note that all equations except for the last one are mod $\varpi$ reductions of equations in [LLHLM18, Section 5.3.3]. We explain how to justify the last equation from the computations in [LLHLM18, Section 5.3.3], which was implicit in [LLHLM18, Table 7]. For the remainder of this paragraph, we adopt the notation of loc. cit. We have $c_{12}\left((a-b) c_{31} c_{23}^{\prime}+(b-c) c_{21}^{*} c_{33}^{\prime}\right)=p z^{*}$. From the equation $c_{32}^{*} c_{13}-p c_{32}^{*} c_{13}^{*}-c_{33}^{\prime} c_{12}=0$, replacing $p$ by $\left(z^{*}\right)^{-1}\left(c_{12}\left((a-b) c_{31} c_{23}^{\prime}+(b-\right.\right.$ c) $\left.c_{21}^{*} c_{33}^{\prime}\right)$ ), we deduce that $c_{13}$ is a certain multiple of $c_{12}$. Finally, using the equation $c_{12} c_{23}=c_{22} c_{13}$ and canceling out $c_{12}$ (as it is a unit after inverting $p$ ), we get an equation solving $c_{23}$ in terms of the remaining variables. The mod $\varpi$ reduction of this equation gives the last equation above.

The minimal primes of $\bar{R}_{\overline{\mathfrak{M}}^{\mathrm{exp}}, \widetilde{w}_{f-1-i}}$ are

$$
\mathfrak{c}_{(0,0)}=\left(c_{12}, c_{22}\right) ; \quad \mathfrak{c}_{\left(\varepsilon_{1}, 1\right)}=\left(c_{12}, c_{31}\right) ; \quad \mathfrak{c}_{\left(\varepsilon_{1}-\varepsilon_{2}, 0\right)}=\left(c_{31}, d_{33}\right)
$$

and

$$
\mathfrak{c}_{(0,1)}=\left(c_{22},(b-c) d_{33} c_{21}^{*}+(a-b) c_{31} d_{23}\right) .
$$

(Note that in $\bar{R}_{\overline{\mathfrak{M}}, \widetilde{w}_{f-1-i}}^{\mathrm{expl}, \nabla}$, there are no more relation than those listed in (3.14). Indeed, let $\widetilde{R}$ be the quotient of $\mathbb{F} \llbracket c_{12}, c_{13}, c_{22}, c_{23}, d_{23}, c_{31}, d_{33}, c_{13}^{*}-\left[\bar{c}_{13}^{*}\right]$, $c_{21}^{*}-\left[\bar{c}_{21}^{*}\right], c_{32}^{*}-\left[\bar{c}_{32}^{*}\right] \rrbracket$ by the relations (3.14). Then the discussion above proves that there is a surjection $\widetilde{R} \rightarrow \bar{R}_{\overline{\mathfrak{M}}^{2}, \widetilde{w}_{f-1-i}}^{\operatorname{expl}, \nabla}$. A direct check on the relations (3.14) shows that $\widetilde{R}$ is reduced, equidimensional of the same dimension as $\bar{R}_{\mathfrak{M}, \widetilde{w}_{f-1-i}}^{\operatorname{expl}, \nabla}$, and with the same Hilbert-Samuel multiplicity. Therefore, the surjection is an isomorphism; see Lemma 3.6.11.) We need to perform matching for the ideals $\mathfrak{c}_{(0,0)}, \mathfrak{c}_{\left(\varepsilon_{1}, 1\right)}, \mathfrak{c}_{\left(\varepsilon_{1}-\varepsilon_{2}, 0\right)}, \mathfrak{w}_{(0)} \stackrel{\text { def }}{=} \mathfrak{c}_{(0,1)} \cap \mathfrak{c}_{(0,0)}$. We provide details for the matching of the ideal $\mathfrak{c}_{(0,0)}$ in Table 3. We have

$$
\bar{R}_{\bar{M}, \widetilde{w}_{f-1}}^{\operatorname{expl}, \nabla} / \mathfrak{c}_{(0,0)}=\mathbb{F} \llbracket d_{23}, c_{31}, d_{33}, c_{13}^{*}-\left[\bar{c}_{13}^{*}\right], c_{21}^{*}-\left[\bar{c}_{21}^{*}\right], c_{32}^{*}-\left[\bar{c}_{32}^{*}\right] \rrbracket
$$

and

$$
A^{(f-1-i)} \quad \bmod \mathfrak{c}_{(0,0)}=\left(\begin{array}{ccc}
0 & 0 & v c_{13}^{*} \\
v c_{21}^{*} & 0 & v d_{23} \\
v c_{31} & v c_{32}^{*} & v d_{33}
\end{array}\right) .
$$


On the other hand, $\widetilde{w}_{f-1-i}^{\prime}=\alpha \beta \alpha \gamma t_{1}, \widetilde{z}_{i}^{*}=\alpha \gamma^{+},\left(a^{\prime}, b^{\prime}, c^{\prime}\right)=(c-1, a, b+1)$, and $A^{\prime(f-1-i)}$ has the form

$$
\left(\begin{array}{ccc}
v^{2} c_{11}^{\prime *} & 0 & 0 \\
v^{2} c_{21}^{\prime} & v c_{22}^{\prime *} & 0 \\
v^{2} d_{31}^{\prime} & v c_{32}^{\prime} & c_{33}^{\prime *}
\end{array}\right)
$$

Now we note that $\widetilde{z}_{f-1-i}=t_{(-1,0,1)}(123)$ and

$$
\left(\begin{array}{ccc}
0 & 0 & v c_{13}^{*} \\
v c_{21}^{*} & 0 & v d_{23} \\
v c_{31} & v c_{32}^{*} & v d_{33}
\end{array}\right)=\left(\begin{array}{ccc}
v^{2} c_{11}^{\prime *} & 0 & 0 \\
v^{2} c_{21}^{\prime} & v c_{22}^{\prime *} & 0 \\
v^{2} d_{31}^{\prime} & v c_{32}^{\prime} & c_{33}^{\prime *}
\end{array}\right)\left(\begin{array}{ccc}
0 & 0 & v^{-1} \\
1 & 0 & 0 \\
0 & v & 0
\end{array}\right)
$$

under the isomorphism

$$
\bar{R}_{\overline{\mathfrak{M}}, \widetilde{w}_{f-1-i}}^{\operatorname{expl}, \nabla} / \mathfrak{c}_{(0,0)} \cong \bar{R}_{\overline{\mathfrak{M}}^{\prime}, \widetilde{w}_{f-1-i}^{\prime}}^{\operatorname{expl}, \nabla}
$$

given by the change of variable

$$
\begin{array}{lll}
c_{21}^{*}=c_{22}^{\prime *}, & c_{13}^{*}=c_{11}^{\prime *}, & d_{23}=c_{21}^{\prime}, \\
c_{31}=c_{32}^{\prime}, & c_{32}^{*}=c_{33}^{\prime *}, & d_{33}=d_{31}^{\prime} .
\end{array}
$$

Such a change of variable is allowed, provided the units that are matched with each other agree modulo the maximal ideal because $\bar{c}_{22}^{*}=\bar{c}_{21}^{*}, \bar{c}_{11}^{\prime *}=\bar{c}_{13}^{*}$, and $\bar{c}_{33}^{\prime *}=\bar{c}_{32}^{*}$ as elements of $\mathbb{F}$ (see Remark 3.6.8). Thus, 3.6.7 applies and we are done with this case. The matching for $\mathfrak{c}_{\left(\varepsilon_{1}-\varepsilon_{2}, 0\right)}$ can be performed in a similar fashion.

Next we provide details for the matching of $\mathfrak{c}_{\left(\varepsilon_{1}, 1\right)}$. We have

$$
\bar{R}_{\mathfrak{M}, \widetilde{w}_{f-1-i}}^{\operatorname{expl,\nabla }} / \mathfrak{c}_{\left(\varepsilon_{1}, 1\right)}=\frac{\mathbb{F}\left[c_{22}, c_{23}, d_{23}, d_{33}, c_{13}^{*}-\left[\bar{c}_{13}^{*}\right], c_{21}^{*}-\left[\bar{c}_{21}^{*}\right], c_{32}^{*}-\left[\bar{c}_{32}^{*}\right] \rrbracket\right.}{\left((-1-a+c) c_{23} c_{32}^{*}-(-1-a+b) c_{22} d_{33}\right)} .
$$

Moreover,

$$
A^{(f-1-i)} \quad \bmod \mathfrak{c}_{\left(\varepsilon_{1}, 1\right)}=\left(\begin{array}{ccc}
0 & 0 & v c_{13}^{*} \\
v c_{21}^{*} & c_{22} & c_{23}+v d_{23} \\
0 & v c_{32}^{*} & v d_{33}
\end{array}\right)
$$

On the other hand, $\widetilde{w}_{f-1-i}^{\prime}=\alpha \beta \gamma \beta t_{1}, \widetilde{z}_{i}^{*}=\gamma^{+} \beta,\left(a^{\prime}, b^{\prime}, c^{\prime}\right)=(c-1, a+1, b)$ and $A^{\prime(f-1-i)}$ has the form

$$
A^{\prime(f-1-i)}=\left(\begin{array}{ccc}
v^{2} c_{11}^{\prime *} & 0 & 0 \\
v c_{21}^{\prime}+v^{2} d_{21}^{\prime} & c_{22}^{\prime *} & c_{23}^{\prime} \\
v^{2} c_{31}^{\prime} & 0 & v c_{33}^{\prime *}
\end{array}\right)
$$


The ring $\overline{R_{\mathfrak{M}^{\prime}, \widetilde{w}_{f-1-i}^{\prime}}^{\text {expl, }}}$ is the quotient of $\mathbb{F}\left[c_{21}^{\prime}, d_{21}^{\prime}, c_{23}^{\prime}, c_{31}^{\prime}, c_{11}^{\prime *}-\left[\bar{c}_{11}^{\prime *}\right], c_{22}^{\prime *}-\left[\bar{c}_{22}^{\prime *}\right]\right.$, $\left.c_{33}^{\prime *}-\left[\bar{c}_{33}^{* *}\right]\right]$ by the relation

$$
\left(-1-a^{\prime}+b^{\prime}\right) c_{21}^{\prime} c_{33}^{\prime *}-\left(b^{\prime}-c^{\prime}\right) c_{31}^{\prime} c_{23}^{\prime}=0 .
$$

Now we note that

$$
\left(\begin{array}{ccc}
0 & 0 & v c_{13}^{*} \\
v c_{21}^{*} & c_{22} & c_{23}+v d_{23} \\
0 & v c_{32}^{*} & v d_{33}
\end{array}\right)=\left(\begin{array}{ccc}
v^{2} c_{11}^{\prime *} & 0 & 0 \\
v c_{21}^{\prime}+v^{2} d_{21}^{\prime} & c_{22}^{\prime *} & c_{23}^{\prime} \\
v^{2} c_{31}^{\prime} & 0 & v c_{33}^{\prime *}
\end{array}\right)\left(\begin{array}{ccc}
0 & 0 & v^{-1} \\
v & 0 & 0 \\
0 & 1 & 0
\end{array}\right)
$$

under the isomorphism

$$
\bar{R}_{\overline{\mathfrak{M}}, \widetilde{w}_{f-1-i}}^{\operatorname{expl}, \nabla} / \mathfrak{c}_{\left(\varepsilon_{1}, 1\right)} \cong \bar{R}_{\overline{\mathfrak{M}}^{\prime}, \widetilde{w}_{f-1-i}^{\prime}}^{\operatorname{expl}}
$$

given by the change of variable

$$
\begin{aligned}
& c_{22}=c_{23}^{\prime}, \quad c_{23}=c_{21}^{\prime}, \quad d_{23}=d_{21}^{\prime}, \\
& d_{33}=c_{31}^{\prime}, \quad c_{13}^{*}=c_{11}^{\prime *}, \quad c_{21}^{*}=c_{22}^{\prime *}, \quad c_{32}^{*}=c_{33}^{\prime *} .
\end{aligned}
$$

We finish the matching in this case.

Finally, we explain the matching of the ideal $\mathfrak{w}_{0} \stackrel{\text { def }}{=} \mathfrak{c}_{(0,1)} \cap \mathfrak{c}_{(0,0)}=\left(c_{22}\right)$. We have

$$
\bar{R}_{\mathfrak{M}, \widetilde{w}_{f-1-i}}^{\operatorname{expl,\nabla }} / \mathfrak{w}_{0}=\frac{\mathbb{F} \llbracket c_{12}, d_{23}, c_{31}, d_{33}, c_{13}^{*}-\left[\bar{c}_{13}^{*}\right], c_{21}^{*}-\left[\bar{c}_{21}^{*}\right], c_{32}^{*}-\left[\bar{c}_{32}^{*}\right] \rrbracket}{\left(c_{12}\left((b-c) d_{33} c_{21}^{*}+(a-b) c_{31} d_{23}\right)\right)} .
$$

Moreover,

$$
A^{(f-1-i)} \bmod \mathfrak{w}_{0}=\left(\begin{array}{ccc}
c_{31} c_{12}\left(c_{32}^{*}\right)^{-1} & c_{12} & d_{33} c_{12}\left(c_{32}^{*}\right)^{-1}+v c_{13}^{*} \\
v c_{21}^{*} & 0 & v d_{23} \\
v c_{31} & v c_{32}^{*} & v d_{33}
\end{array}\right) .
$$

On the other hand, $\widetilde{w}_{f-1-i}^{\prime}=\alpha \beta \alpha t_{1}, \widetilde{z}_{i}^{*}=\alpha,\left(a^{\prime}, b^{\prime}, c^{\prime}\right)=(b, a, c)$ and $A^{\prime(f-1-i)}$ has the form

$$
A^{\prime(f-1-i)}=\left(\begin{array}{ccc}
c_{11}^{\prime} & c_{11}^{\prime} c_{32}^{\prime}\left(c_{31}^{\prime *}\right)^{-1} & d_{33}^{\prime} c_{11}^{\prime}\left(c_{31}^{\prime *}\right)^{-1}+v c_{13}^{\prime *} \\
0 & v c_{22}^{\prime *} & v c_{23}^{\prime} \\
v c_{31}^{\prime *} & v c_{32}^{\prime} & v d_{33}^{\prime}
\end{array}\right)
$$

The ring $\bar{R}_{\overline{\mathfrak{M}}^{\prime}, \widetilde{w}_{f-1-i}^{\prime}}^{\mathrm{expl}, \nabla}$ is the quotient of $\mathbb{F}\left[c_{11}^{\prime}, c_{23}^{\prime}, c_{32}^{\prime}, d_{33}^{\prime}, c_{31}^{*}-\left[\bar{c}_{31}^{*}\right], c_{22}^{*}-\left[\bar{c}_{22}^{*}\right]\right.$, $c_{13}^{*}-\left[\bar{c}_{13}^{*}\right] \|$ by the relations

$$
c_{11}^{\prime}\left(\left(a^{\prime}-b^{\prime}\right) c_{23}^{\prime} c_{32}^{\prime}-\left(a^{\prime}-c^{\prime}\right) c_{33}^{\prime *} d_{33}^{\prime}\right)=0 .
$$


(We remark that the form of the $(3,3)$ entry of $A^{\prime(f-1-i)}$ is as above because in the notation of [LLHLM18, Section 5.3.1], we have $c_{33}=-y_{33}^{\prime} c_{13} c_{31}^{*}=0$ $\bmod p$ since $c_{13} c_{31}^{*}=c_{11} c_{33}^{\prime} \bmod p$ and $y_{33}^{\prime} c_{11}=0 \bmod p$.)

Now we note that

$$
\begin{aligned}
& \left(\begin{array}{ccc}
c_{31} c_{12}\left(c_{32}^{*}\right)^{-1} & c_{12} & d_{33} c_{12}\left(c_{32}^{*}\right)^{-1}+v c_{13}^{*} \\
v c_{21}^{*} & 0 & v d_{23} \\
v c_{31} & v c_{32}^{*} & v d_{33}
\end{array}\right)= \\
& =\left(\begin{array}{ccc}
c_{11}^{\prime} & c_{11}^{\prime} c_{32}^{\prime}\left(c_{31}^{\prime *}\right)^{-1} & d_{33}^{\prime} c_{11}^{\prime}\left(c_{31}^{* *}\right)^{-1}+v c_{13}^{\prime *} \\
0 & v c_{22}^{\prime *} & v c_{23}^{\prime} \\
v c_{31}^{\prime *} & v c_{32}^{\prime} & v d_{33}^{\prime}
\end{array}\right)\left(\begin{array}{lll}
0 & 1 & 0 \\
1 & 0 & 0 \\
0 & 0 & 1
\end{array}\right)
\end{aligned}
$$

under the isomorphism

$$
\bar{R}_{\overline{\mathfrak{M}}, \widetilde{w}_{f-1-i}}^{\operatorname{expl,\nabla }} / \mathfrak{w}_{0} \cong \bar{R}_{\overline{\mathfrak{M}}^{\prime}, \widetilde{w}_{f-1-i}^{\prime}}^{\operatorname{expl},}
$$

given by the change of variable

$$
\begin{aligned}
& c_{12}=c_{11}^{\prime}, \quad c_{31}=c_{32}^{\prime}, \quad d_{23}=d_{23}^{\prime}, \\
& d_{33}=d_{33}^{\prime}, \quad c_{32}^{*}=c_{31}^{\prime *}, \quad c_{21}^{*}=c_{22}^{\prime *}, \quad c_{13}^{*}=c_{13}^{\prime *} \text {. }
\end{aligned}
$$

Case $\widetilde{w}_{f-1-i}=\alpha t_{\underline{1}}$. From [LLHLM18, Table 5], the matrix of the partial Frobenius $A^{(f-1-i)}$ has the following form:

$$
\left(\begin{array}{ccc}
c_{11} & c_{12}+v c_{12}^{*} & c_{13} \\
v c_{21}^{*} & c_{22}+v d_{22} & c_{23} \\
v c_{31} & v c_{32} & c_{33}+v c_{33}^{*}
\end{array}\right) .
$$

Set $\widetilde{c}_{32} \stackrel{\text { def }}{=} \frac{c_{32} c_{21}^{*}-d_{22} c_{31}}{c_{21}^{*}}$. Recall from item (2) above that $\bar{R}_{\overline{\mathfrak{M}}_{,}, \widetilde{w}_{f-1-i}}^{\operatorname{expl}, \nabla}$ is the quotient of

$$
\mathbb{F} \llbracket c_{11}, c_{12}, c_{13}, c_{22}, c_{23}, c_{31}, \widetilde{c}_{32}, c_{33}, d_{22}, c_{12}^{*}-\left[\bar{c}_{12}^{*}\right], c_{21}^{*}-\left[\bar{c}_{21}^{*}\right], c_{33}^{*}-\left[\bar{c}_{33}^{*}\right] \rrbracket
$$

by the following relations:

$$
\begin{array}{ll}
c_{11} c_{23}=0, & c_{33}^{*} c_{11} \widetilde{c}_{32}=c_{13} c_{31} \widetilde{c}_{32}, \\
c_{11} d_{22} c_{33}^{*}=\frac{b-c}{a-b} c_{21}^{*} c_{13} \widetilde{c}_{32}, & c_{13} c_{23} \widetilde{c}_{32}=0, \\
c_{23} c_{31} \widetilde{c}_{32}=0, & c_{21}^{*} c_{33}=c_{31} c_{23}, \\
c_{12} c_{33}^{*}=\frac{a-c}{a-b} c_{13} \widetilde{c}_{32}, & c_{22} c_{33}^{*}=\frac{(-1-a+c)}{(-1-a+b)} c_{23} \widetilde{c}_{32},
\end{array}
$$


and

$$
(a-b) c_{13} c_{31} d_{22}+(c-b) c_{13} \widetilde{c}_{32} c_{21}^{*}+(-1-a+c) c_{23} c_{31} c_{12}^{*}=0 .
$$

(Note that $\overline{R_{\mathfrak{M}} \text { expl, }, \widetilde{w}_{f-1-i}}$ is formally smooth of relative dimension three over the ring $\widetilde{R}$ defined in [LLHLM18, Proposition 8.11].)

We provide details for the matching of the ideal $\mathfrak{c}_{\left(\varepsilon_{1}, 1\right)}$. We have

$$
\bar{R}_{\overline{\mathfrak{M}}, \widetilde{w}_{f-1-i}}^{\operatorname{expl},} / \mathfrak{c}_{\left(\varepsilon_{1}, 1\right)}=\frac{\mathbb{F} \llbracket c_{22}, c_{23}, c_{32}, d_{22}, c_{21}^{*}-\left[\bar{c}_{21}^{*}\right], c_{12}^{*}-\left[\bar{c}_{12}^{*}\right], c_{33}^{*}-\left[\bar{c}_{33}^{*}\right] \rrbracket}{\left((-1-a+b) c_{22} c_{33}^{*}-(-1-a+c) c_{23} c_{32}\right)}
$$

and

$$
A^{(f-1-i)} \quad \bmod \mathfrak{c}_{\left(\varepsilon_{1}, 1\right)}=\left(\begin{array}{ccc}
0 & v c_{12}^{*} & 0 \\
v c_{21}^{*} & c_{22}+v d_{22} & c_{23} \\
0 & v c_{32} & v c_{33}^{*}
\end{array}\right) .
$$

On the other hand, $\widetilde{w}_{f-1-i}^{\prime}=\alpha \beta \gamma \beta, \widetilde{z}_{i}^{*}=\beta \gamma^{+} \beta,\left(a^{\prime}, b^{\prime}, c^{\prime}\right)=(b-1, a+1, c)$ and $A^{\prime(f-1-i)}$ has the form

$$
\left(\begin{array}{ccc}
v^{2} c_{11}^{\prime *} & 0 & 0 \\
v\left(c_{21}^{\prime}+v d_{21}^{\prime}\right) & c_{22}^{\prime *} & c_{23}^{\prime} \\
v^{2} c_{31}^{\prime} & 0 & v c_{33}^{\prime *}
\end{array}\right)
$$

The ring $\bar{R}_{\overline{\mathfrak{M}}^{\prime}, \widetilde{w}_{f-1-i}^{\prime}}^{\mathrm{expl} \nabla}$ is the quotient of $\mathbb{F}\left[c_{21}^{\prime}, d_{21}^{\prime}, c_{23}^{\prime}, c_{31}^{\prime}, c_{11}^{\prime *}-\left[\bar{c}_{11}^{\prime *}\right], c_{22}^{\prime *}-\left[\bar{c}_{22}^{\prime *}\right]\right.$, $\left.c_{33}^{\prime *}-\left[\bar{c}_{33}^{\prime *}\right]\right]$ by the relation

$$
\left(-1-a^{\prime}+b^{\prime}\right) c_{21}^{\prime} c_{33}^{\prime *}-\left(b^{\prime}-c^{\prime}\right) c_{31}^{\prime} c_{23}^{\prime}=0 .
$$

We now note that

$$
\left(\begin{array}{ccc}
0 & v c_{12}^{*} & 0 \\
v c_{21}^{*} & c_{22}+v d_{22} & c_{23} \\
0 & v c_{32} & v c_{33}^{*}
\end{array}\right)=\left(\begin{array}{ccc}
v^{2} c_{11}^{\prime *} & 0 & 0 \\
v\left(c_{21}^{\prime}+v d_{21}^{\prime}\right) & c_{22}^{\prime *} & c_{23}^{\prime} \\
v^{2} c_{31}^{\prime} & 0 & v c_{33}^{\prime *}
\end{array}\right)\left(\begin{array}{ccc}
0 & v^{-1} & 0 \\
v & 0 & 0 \\
0 & 0 & 1
\end{array}\right)
$$

under the isomorphism

$$
\bar{R}_{\overline{\mathfrak{M}}, \widetilde{w}_{f-1-i}}^{\operatorname{expl}, \nabla} / \mathfrak{c}_{\left(\varepsilon_{1}, 1\right)} \cong \bar{R}_{\overline{\mathfrak{M}}^{\prime}, \widetilde{w}_{f-1-i}^{\prime}}^{\operatorname{expl},}
$$

given by the change of variables

$$
\begin{array}{lll}
c_{12}^{*}=c_{11}^{\prime *}, & c_{21}^{*}=c_{22}^{\prime *}, & d_{22}=d_{21}^{\prime} \\
c_{23}=c_{23}^{\prime} & c_{32}=c_{31}^{\prime} & c_{33}^{*}=c_{33}^{\prime *} .
\end{array}
$$


We now explain the matching of the ideal $\mathfrak{w}_{\varepsilon_{2}} \stackrel{\text { def }}{=} \mathfrak{c}_{\left(\varepsilon_{2}, 0\right)} \cap \mathfrak{c}_{\left(\varepsilon_{2}, 1\right)}=\left(c_{11}, \widetilde{c}_{32}\right)$. We have

$$
\bar{R}_{\mathfrak{M}, \widetilde{w}_{f-1-i}}^{\operatorname{expl} \nabla} / \mathfrak{w}_{\varepsilon_{2}}=\frac{\mathbb{F} \llbracket c_{13}, c_{23}, c_{31}, d_{22}, c_{12}^{*}-\left[\bar{c}_{12}^{*}\right], c_{21}^{*}-\left[\bar{c}_{21}^{*}\right], c_{33}^{*}-\left[\bar{c}_{33}^{*} \rrbracket\right.}{c_{31}\left((a-b) c_{13} d_{22}+(-1-a+c) c_{12}^{*} c_{23}\right)} .
$$

Moreover,

$$
A^{(f-1-i)} \quad \bmod \mathfrak{w}_{\varepsilon_{2}}=\left(\begin{array}{ccc}
0 & v c_{12}^{*} & c_{13} \\
v c_{21}^{*} & v d_{22} & c_{23} \\
v c_{31} & v d_{22} c_{31}\left(c_{21}^{*}\right)^{-1} & c_{23} c_{31}\left(c_{21}^{*}\right)^{-1}+v c_{33}^{*}
\end{array}\right) .
$$

On the other hand, $\widetilde{w}_{f-1-i}^{\prime}=\alpha \gamma \alpha, \widetilde{z}_{i}^{*}=\gamma^{+} \alpha$ and $\left(a^{\prime}, b^{\prime}, c^{\prime}\right)=(b, c-1, a+1)$ and $A^{\prime(f-1-i)}$ has the form

$$
\left(\begin{array}{ccc}
v c_{11}^{\prime *} & v c_{12}^{\prime} & 0 \\
v c_{21}^{\prime} & v d_{22}^{\prime} & c_{23}^{\prime *} \\
v c_{33}^{\prime} c_{21}^{\prime}\left(c_{23}^{\prime *}\right)^{-1} & v\left(c_{33}^{\prime} d_{22}^{\prime}\left(c_{23}^{\prime *}\right)^{-1}+v c_{32}^{\prime *}\right) & c_{33}^{\prime}
\end{array}\right)
$$

The ring $\frac{\bar{R}_{\mathfrak{M}^{\prime}, \widetilde{w}_{f-1-i}^{\prime}}^{\text {expl, }}}{\text { in }}$ is the quotient of $\mathbb{F}\left[c_{12}^{\prime}, c_{21}^{\prime}, d_{22}^{\prime}, c_{33}^{\prime}, c_{11}^{\prime *}-\left[\bar{c}_{11}^{\prime *}\right], c_{23}^{\prime *}-\left[\bar{c}_{23}^{\prime *}\right]\right.$, $\left.c_{32}^{\prime *}-\left[\bar{c}_{32}^{\prime *}\right]\right]$ by the relation

$$
c_{33}^{\prime}\left(\left(-1-a^{\prime}+c^{\prime}\right) c_{12}^{\prime} c_{21}^{\prime}-\left(1-b^{\prime}+c^{\prime}\right) c_{11}^{\prime *} d_{22}^{\prime}\right)=0 .
$$

Now we note that

$$
\begin{aligned}
& \left(\begin{array}{ccc}
0 & v c_{12}^{*} & c_{13} \\
v c_{21}^{*} & v d_{22} & c_{23} \\
v c_{31} & v d_{22} c_{31}\left(c_{21}^{*}\right)^{-1} & c_{23} c_{31}\left(c_{21}^{*}\right)^{-1}+v c_{33}^{*}
\end{array}\right) \\
& =\left(\begin{array}{ccc}
v c_{11}^{\prime *} & v c_{12}^{\prime} & 0 \\
v c_{21}^{\prime} & v d_{22}^{\prime} & c_{23}^{\prime *} \\
v c_{33}^{\prime} c_{21}^{\prime}\left(c_{23}^{\prime *}\right)^{-1} & v\left(c_{33}^{\prime} d_{22}^{\prime}\left(c_{23}^{\prime *}\right)^{-1}+v c_{32}^{\prime *}\right) & c_{33}^{\prime}
\end{array}\right)\left(\begin{array}{ccc}
0 & 1 & 0 \\
0 & 0 & v^{-1} \\
v & 0 & 0
\end{array}\right)
\end{aligned}
$$

under the isomorphism

$$
\bar{R}_{\overline{\mathfrak{M}}, \widetilde{w}_{f-1-i}}^{\operatorname{expl}, \nabla} / \mathfrak{w}_{\varepsilon_{2}} \cong \bar{R}_{\overline{\mathfrak{M}}^{\prime}, \widetilde{w}_{f-1-i}^{\prime}}^{\operatorname{expl} \nabla}
$$

given by the change of variables

$$
\begin{aligned}
& c_{21}^{*}=c_{23}^{\prime *}, \quad c_{31}=c_{33}^{\prime}, \quad c_{12}^{*}=c_{11}^{\prime *} \\
& d_{22}=c_{21}^{\prime}, \quad c_{13}=c_{12}^{\prime}, \quad c_{23}=d_{22}^{\prime}, \quad c_{33}^{*}=c_{32}^{\prime *} \text {. }
\end{aligned}
$$


Case $\widetilde{w}_{f-1-i}=t_{1}$. From [LLHLM18, Table 5], the matrix of the partial Frobenius $A^{(f-1-i)}$ has the following form:

$$
\left(\begin{array}{ccc}
c_{11}+v c_{11}^{*} & c_{12} & c_{13} \\
v c_{21} & c_{22}+v c_{22}^{*} & c_{23} \\
v c_{31} & v c_{32} & c_{33}+v c_{33}^{*}
\end{array}\right) .
$$

Recall from item (3) above that $\bar{R} \frac{\operatorname{expl}, \nabla}{\mathfrak{M}_{f-1-i}}$ is the quotient of $\mathbb{F} \llbracket c_{i j}, c_{k k}^{*}-\left[\bar{c}_{k k}^{*}\right]$, $1 \leqslant i, j, k \leqslant 3$ ॥ by the relations

$$
\begin{array}{lll}
c_{i i} c_{j j}=0 \quad \text { for } i \neq j, & c_{11} c_{23}=0, & \\
c_{33} c_{12}=0, & c_{12} c_{23}=c_{22} c_{13}, & c_{11} c_{32}=c_{12} c_{31}, \\
c_{21} c_{33}=c_{31} c_{23}, & &
\end{array}
$$

and

$$
\begin{aligned}
& (-1-a+c) c_{22}^{*} c_{33}+(-1-a+b) c_{22} c_{33}^{*}-(-1-a+c) c_{23} c_{32}=0 \\
& (a-b) c_{33}^{*} c_{11}+(-1-b+c) c_{33} c_{11}^{*}-(a-b) c_{13} c_{31}=0 \\
& (b-c) c_{11}^{*} c_{22}+(a-c) c_{11} c_{22}^{*}-(b-c) c_{12} c_{21}=0 \\
& c_{11} c_{22}^{*} c_{33}^{*}+c_{22} c_{11}^{*} c_{33}^{*}+c_{33} c_{11}^{*} c_{22}^{*}-c_{11}^{*} c_{23} c_{32}-c_{22}^{*} c_{13} c_{31} \\
& \quad-c_{33}^{*} c_{12} c_{21}+c_{13} c_{32} c_{21}=0 .
\end{aligned}
$$

(Note that $\bar{R}_{\mathfrak{M}, \widetilde{w}_{f-1-i}}^{\text {expl, }}$ is formally smooth of relative dimension three over the ring $\widetilde{R}$ defined in [LLHLM18, Corollary 8.4].)

We provide details for the matching of $\mathfrak{c}_{(0,0)}=\left(c_{11}, c_{22}, c_{33}, c_{13}, c_{23}, c_{12}\right)$.

We have

$$
\bar{R}_{\mathfrak{M}, \widetilde{w}_{f-1-i}}^{\operatorname{expl}, \nabla} / \mathfrak{c}_{(0,0)}=\mathbb{F} \llbracket c_{i j}, c_{k k}^{*}-\left[\bar{c}_{k k}^{*}\right], 1 \leqslant j<i \leqslant 3,1 \leqslant k \leqslant 3 \rrbracket
$$

and

$$
A^{(f-1-i)} \quad \bmod \mathfrak{c}_{(0,0)}=\left(\begin{array}{ccc}
v c_{11}^{*} & 0 & 0 \\
v c_{21} & v c_{22}^{*} & 0 \\
v c_{31} & v c_{32} & v c_{33}^{*}
\end{array}\right) .
$$

On the other hand, $\widetilde{w}_{f-1-i}^{\prime}=\alpha \beta \alpha \gamma t_{1}$ and $A^{\prime(f-1-i)}$ has the form

$$
\left(\begin{array}{ccc}
v^{2} c_{11}^{\prime *} & 0 & 0 \\
v^{2} c_{21}^{\prime} & v c_{22}^{\prime *} & 0 \\
v^{2} c_{31}^{\prime} & v c_{32}^{\prime} & c_{33}^{\prime *}
\end{array}\right)
$$


We note that $\widetilde{z}_{f-1-i}=t_{(1,0,-1)}$ and

$$
\left(\begin{array}{ccc}
v c_{11}^{*} & 0 & 0 \\
v c_{21} & v c_{22}^{*} & 0 \\
v c_{31} & v c_{32} & v c_{33}^{*}
\end{array}\right)=\left(\begin{array}{ccc}
v^{2} c_{11}^{\prime *} & 0 & 0 \\
v^{2} c_{21}^{\prime} & v c_{22}^{\prime *} & 0 \\
v^{2} c_{31}^{\prime} & v c_{32}^{\prime} & c_{33}^{\prime *}
\end{array}\right)\left(\begin{array}{ccc}
v^{-1} & 0 & 0 \\
0 & 1 & 0 \\
0 & 0 & v
\end{array}\right)
$$

under the isomorphism

$$
\bar{R}_{\mathfrak{M}, \widetilde{w}_{f-1-i}}^{\operatorname{expl,\nabla }} / \mathfrak{c}_{(0,0)} \cong \bar{R}_{\overline{\mathfrak{M}}^{\prime}, \widetilde{w}_{f-1-i}^{\prime}}^{\operatorname{expl},}
$$

given by the change of variables

$$
c_{i j}=c_{i j}^{\prime} \quad \text { for } 1 \leqslant j<i \leqslant 3, \quad c_{k k}^{*}=c_{k k}^{\prime *} \quad \text { for } 1 \leqslant k \leqslant 3 .
$$

We now explain the matching for the ideal $\mathfrak{w}_{0} \stackrel{\text { def }}{=} \mathfrak{c}_{(0,0)} \cap \mathfrak{c}_{(0,1)}=\left(c_{22}, c_{33}, c_{23}\right.$, $\left.c_{33}^{*} c_{12}-c_{13} c_{32}\right)$. We have

$$
\bar{R}_{\mathfrak{M}, \widetilde{w}_{f-1-i}}^{\operatorname{expl}, \nabla} / \mathfrak{w}_{0}=\frac{\mathbb{F} \llbracket c_{13}, c_{21}, c_{31}, c_{32}, c_{11}^{*}-\left[\bar{c}_{11}^{*}\right], c_{22}^{*}-\left[\bar{c}_{22}^{*}\right], c_{33}^{*}-\left[\bar{c}_{33}^{*}\right] \rrbracket}{\left(c_{13}\left((a-c) c_{22}^{*} c_{31}-(b-c) c_{32} c_{21}\right)\right)}
$$

(we have used the relations

$$
c_{33}^{*} c_{12}-c_{13} c_{32}=0, \quad c_{33}^{*} c_{11}-c_{13} c_{31}=0, \quad(a-c) c_{22}^{*} c_{11}-(b-c) c_{12} c_{21}=0
$$

holding in $\bar{R}_{\mathfrak{M}, \widetilde{w}_{f-1-i}}^{\text {expl, }} / \mathfrak{w}_{0}$ ). Moreover,

$$
A^{(f-1-i)} \bmod \mathfrak{w}_{0}=\left(\begin{array}{ccc}
c_{13} c_{31}\left(c_{33}^{*}\right)^{-1}+v c_{11}^{*} & c_{13} c_{32}\left(c_{33}^{*}\right)^{-1} & c_{13} \\
v c_{21} & v c_{22}^{*} & 0 \\
v c_{31} & v c_{32} & v c_{33}^{*}
\end{array}\right) .
$$

On the other hand, $\widetilde{w}_{f-1-i}=\alpha \beta \alpha=\widetilde{z}_{i}^{*},\left(a^{\prime}, b^{\prime}, c^{\prime}\right)=(c, b, a)$ and $A^{\prime(f-1-i)}$ has the form

$$
\left(\begin{array}{ccc}
c_{11}^{\prime} & c_{11}^{\prime} c_{32}^{\prime}\left(c_{31}^{\prime *}\right)^{-1} & c_{11}^{\prime} d_{33}^{\prime}\left(c_{31}^{\prime *}\right)^{-1}+v c_{13}^{\prime *} \\
0 & v c_{22}^{\prime *} & v c_{23}^{\prime} \\
v c_{31}^{\prime *} & v c_{32}^{\prime} & v d_{33}^{\prime}
\end{array}\right)
$$

The ring $R_{\overline{\mathfrak{M}}^{\prime}, \widetilde{w}_{f-1-i}^{\prime}}^{\operatorname{expl}, \nabla}$ is the quotient of $\mathbb{F}\left[c_{11}^{\prime}, c_{23}^{\prime}, c_{32}^{\prime}, d_{33}^{\prime}, c_{13}^{\prime *}-\left[\bar{c}_{13}^{\prime *}\right], c_{22}^{\prime *}-\left[\bar{c}_{22}^{\prime *}\right]\right.$, $\left.c_{31}^{\prime *}-\left[\bar{c}_{31}^{\prime *}\right]\right]$ by the relation

$$
c_{11}^{\prime}\left(\left(a^{\prime}-b^{\prime}\right) c_{23}^{\prime} c_{32}^{\prime}-\left(a^{\prime}-c^{\prime}\right) c_{22}^{\prime *} d_{33}^{\prime}\right)=0 .
$$

We now note that

$$
\left(\begin{array}{ccc}
c_{13} c_{31}\left(c_{33}^{*}\right)^{-1}+v c_{11}^{*} & c_{13} c_{32}\left(c_{33}^{*}\right)^{-1} & c_{13} \\
v c_{21} & v c_{22}^{*} & 0 \\
v c_{31} & v c_{32} & v c_{33}^{*}
\end{array}\right)
$$




$$
=\left(\begin{array}{ccc}
c_{11}^{\prime} & c_{11}^{\prime} c_{32}^{\prime}\left(c_{31}^{\prime *}\right)^{-1} & c_{11}^{\prime} d_{33}^{\prime}\left(c_{31}^{\prime *}\right)^{-1}+v c_{13}^{\prime *} \\
0 & v c_{22}^{\prime *} & v c_{23}^{\prime} \\
v c_{31}^{\prime *} & v c_{32}^{\prime} & v d_{33}^{\prime}
\end{array}\right)\left(\begin{array}{ccc}
0 & 0 & 1 \\
0 & 1 & 0 \\
1 & 0 & 0
\end{array}\right)
$$

under the isomorphism

$$
\bar{R}_{\overline{\mathfrak{M}}, \widetilde{w}_{f-1-i}}^{\operatorname{expl}, \nabla} / \mathfrak{w}_{0} \cong \bar{R} \frac{\overline{\mathfrak{M}}^{\operatorname{expl}}, \nabla}{\widetilde{w}_{f-1-i}^{\prime}}
$$

given by the change of variables

$$
\begin{array}{lll}
c_{13}=c_{11}^{\prime}, & c_{21}=c_{23}^{\prime}, & c_{31}=d_{33}^{\prime}, \\
c_{32}=c_{32}^{\prime}, & c_{11}^{*}=c_{13}^{\prime *}, & c_{22}^{*}=c_{22}^{\prime *},
\end{array}
$$

Case $\widetilde{w}_{f-1-i}=\beta \alpha t_{\underline{1}}$ and $\alpha \beta \alpha t_{\underline{1}}$ : The computations of these two cases are very similar to those we already performed and are left to the reader.

3.6.3. Ideal relations in deformation rings. In this subsection, we collect some results about sums of intersections of minimal primes in the potentially crystalline deformation rings $R_{\bar{\rho}}^{\tau}$. These computations play a crucial role in Section 5.1, where they are used to compute the value of a patching functor on certain representations as the limit of the value of the patching functor on simpler pieces of the representation.

Thanks to Theorem 3.6.4, all computations that we need to perform can be done on the rings $\bar{R}_{\overline{\mathfrak{M}}, \widetilde{w}_{f-1-i}}^{\text {expl, } \nabla}$ given in Table 3 . We continue to adopt the notation and setting of Theorem 3.6.4.

We will frequently make use of the following.

Lemma 3.6.11. Suppose we have a surjection of rings $g: S \rightarrow R$. Assume that $R$ and $S$ are equidimensional of dimension $d$, have the same number of minimal primes, and $S$ is reduced. Then $g$ is an isomorphism.

Proof. The first two hypotheses imply that the kernel of $g$ is nilpotent since $g$ induces an isomorphism between the underlying topological spaces of $\operatorname{Spec}(R)$ and $\operatorname{Spec}(S)$. But since $S$ is reduced, this kernel must in fact be 0 .

Ideal relations in $\bar{R}_{\bar{M}, \text { id }}^{\exp , \nabla}$

LEMMA 3.6.12. In the ring $\bar{R}_{\overline{\mathfrak{M}} \text {,id }}^{\mathrm{expl}, \nabla}$, we have

$$
\left(\mathfrak{w}_{0} \cap \mathfrak{c}_{\left(\varepsilon_{1}, 0\right)}\right)+\left(\mathfrak{w}_{0} \cap \mathfrak{c}_{\left(\varepsilon_{2}, 0\right)}\right)=\mathfrak{w}_{0} .
$$


Proof. An elementary check on the list of generators of the ideals $\mathfrak{w}_{0}, \mathfrak{c}_{\left(\varepsilon_{1}, 0\right)}$ and $\mathfrak{c}_{\left(\varepsilon_{2}, 0\right)}$ (cf. Table 3) shows that

$$
\mathfrak{w}_{0} \cap \mathfrak{c}_{\left(\varepsilon_{1}, 0\right)} \supseteq\left(c_{33}, c_{23}, c_{22}\right), \quad \mathfrak{w}_{0} \cap \mathfrak{c}_{\left(\varepsilon_{2}, 0\right)} \supseteq\left(c_{33}, c_{22}, c_{13} c_{32}-c_{12} c_{33}^{*}\right) .
$$

In particular (again by looking at the list of generators of $\mathfrak{w}_{0}$ ), we have $\left(\mathfrak{w}_{0} \cap\right.$ $\left.\mathfrak{c}_{\left(\varepsilon_{1}, 0\right)}\right)+\left(\mathfrak{w}_{0} \cap \mathfrak{c}_{\left(\varepsilon_{2}, 0\right)}\right) \supseteq \mathfrak{w}_{0}$. The reverse inclusion is obvious.

LEMMA 3.6.13. In the ring $\bar{R}_{\overline{\mathfrak{M}} \text {,id }}^{\mathrm{expl}, \nabla}$, we have

$$
\mathfrak{w}_{0} \cap \mathfrak{c}_{\left(\varepsilon_{1}, 0\right)} \cap \mathfrak{c}_{\left(\varepsilon_{2}, 0\right)}=\left(c_{22}, c_{33}\right) .
$$

Proof. We let $\widetilde{R}$ be the ring with the same presentation as $\bar{R}_{\overline{\mathfrak{M}} \text {,id }}^{\text {exl, }}$ except that all $c_{i j}^{*}$ are set to 1 . As explained in [LLHLM18, Corollary 8.4], there is a natural identification of $\overline{R_{\bar{M}} \text {,id }}$ expl, with the power series over $\widetilde{R}$ with three variables, and we can work with the ring $\widetilde{R}$ instead of $\bar{R}_{\overline{\mathfrak{M}} \text {,id }}^{\text {expl, }}$. All the ideals that we consider come from $\widetilde{R}$ and are given by generators with the same name.

From Table 3, we immediately obtain $\left(c_{22}, c_{33}\right) \subseteq \mathfrak{w}_{0} \cap \mathfrak{c}_{\left(\varepsilon_{1}, 0\right)} \cap \mathfrak{c}_{\left(\varepsilon_{2}, 0\right)}$, and, hence, we need to prove that the surjection

$$
\widetilde{R} /\left(c_{22}, c_{33}\right) \rightarrow \widetilde{R} /\left(\mathfrak{w}_{0} \cap \mathfrak{c}_{\left(\varepsilon_{1}, 0\right)} \cap \mathfrak{c}_{\left(\varepsilon_{2}, 0\right)}\right)
$$

is an isomorphism. The ring on the right-hand side is equidimensional of dimension three and has four minimal primes. By Lemma 3.6.11, it suffices to show that $\widetilde{R} /\left(c_{22}, c_{33}\right)$ is reduced, is equidimensional of dimension three, and has four minimal primes.

Now $\widetilde{R} /\left(c_{22}, c_{33}\right)$ is the quotient of the power series ring $\mathbb{F} \llbracket c_{11}, c_{i j}, 1 \leqslant i, j$, $\leqslant 3, i \neq j \rrbracket$ by the ideal generated by the following elements:

$$
\begin{array}{lll}
c_{11} c_{23}, & c_{12} c_{23}, & c_{11} c_{32}-c_{12} c_{31}, \\
c_{31} c_{23}, & c_{23} c_{32}, & \kappa c_{11}=c_{21} c_{12}, \\
c_{11}-c_{13} c_{31}, & c_{11}-c_{12} c_{21}-c_{13} c_{31}-c_{23} c_{32}+c_{21} c_{13} c_{32} &
\end{array}
$$

for some $\kappa \in \mathbb{F}^{\times}$depending on $(a, b, c)$. By standard manipulations, we conclude that $\widetilde{R} /\left(c_{22}, c_{33}\right)$ is isomorphic to the quotient of the power series ring $\mathbb{F} \llbracket c_{i j}$, $1 \leqslant i, j, \leqslant 3, i \neq j \rrbracket$ by the ideal generated by the following elements:

$$
\begin{array}{lll}
c_{31} c_{23}, & c_{23} c_{32}, & c_{12} c_{23}, \\
c_{31}\left(c_{12}-c_{13} c_{32}\right), & c_{13}\left(\kappa c_{31}-c_{21} c_{32}\right), & c_{21}\left(c_{12}-c_{13} c_{32}\right) ;
\end{array}
$$


hence, by writing $\widetilde{c}_{12} \stackrel{\text { def }}{=} c_{12}-c_{13} c_{32}, \widetilde{c}_{31} \stackrel{\text { def }}{=} \kappa c_{31}-c_{21} c_{32}$, we obtain

$$
\widetilde{R} /\left(c_{22}, c_{33}\right) \cong \frac{\mathbb{F} \llbracket \widetilde{c}_{12}, \widetilde{c}_{31}, c_{23}, c_{13}, c_{21}, c_{32} \rrbracket}{\left(\widetilde{c}_{12} \widetilde{c}_{31}, \widetilde{c}_{12} c_{23}, \widetilde{c}_{12} c_{21}, \widetilde{c}_{31} c_{23}, \widetilde{c}_{31} c_{13}, c_{23} c_{32}\right)}
$$

This latter ring is easily seen to be equidimensional of dimension three and has four minimal primes. It is furthermore reduced since it is the quotient of a power series ring by an ideal generated by square-free monomials.

From Lemma 3.6.13, the same argument used in the proof of Lemma 3.6.12 gives the following.

LEMMA 3.6.14. In the ring $\bar{R}_{\overline{\mathfrak{M}} \text {,id }}^{\operatorname{expl}, \nabla}$, we have

$$
\left(\mathfrak{w}_{0} \cap \mathfrak{w}_{\varepsilon_{1}} \cap \mathfrak{c}_{\left(\varepsilon_{2}, 0\right)}\right)+\left(\mathfrak{w}_{0} \cap \mathfrak{w}_{\varepsilon_{2}} \cap \mathfrak{c}_{\left(\varepsilon_{1}, 0\right)}\right)=\mathfrak{w}_{0} \cap \mathfrak{c}_{\left(\varepsilon_{1}, 0\right)} \cap \mathfrak{c}_{\left(\varepsilon_{2}, 0\right)}
$$

Proof. From Table 3, we immediately deduce that $\left(\mathfrak{w}_{0} \cap \mathfrak{w}_{\varepsilon_{1}} \cap \mathfrak{c}_{\left(\varepsilon_{2}, 0\right)}\right) \supseteq\left(c_{33}\right)$ and $\left(\mathfrak{w}_{0} \cap \mathfrak{w}_{\varepsilon_{2}} \cap \mathfrak{c}_{\left(\varepsilon_{1}, 0\right)}\right) \supseteq\left(c_{22}\right)$. The conclusion now follows as in Lemma 3.6.12 by noting that $\left(c_{22}, c_{33}\right)=\mathfrak{w}_{0} \cap \mathfrak{c}_{\left(\varepsilon_{1}, 0\right)} \cap \mathfrak{c}_{\left(\varepsilon_{2}, 0\right)}$.

Ideal relations in $\bar{R}_{\mathfrak{M}, \alpha}^{\operatorname{expl}, \nabla}$.

LEMMA 3.6.15. Consider the ring $\bar{R}_{\mathfrak{M}, \alpha}^{\text {expl, }, \nabla}$ (cf. Table 3) and let $I_{\Lambda_{0}} \stackrel{\text { def }}{=} \mathfrak{c}_{(0,0)} \cap$ $\mathfrak{c}_{(0,1)} \cap \mathfrak{c}_{\left(\varepsilon_{2}, 0\right)}$.

Then

(1) $I_{\Lambda_{0}}=\left(c_{11} c_{33}^{*}-c_{13} c_{31}, c_{23} c_{31}, c_{23}\left(c_{32} c_{21}^{*}-d_{22} c_{31}\right)\right)$,

(2) $\mathfrak{c}_{\left(\varepsilon_{1}, 1\right)} \cap \mathfrak{c}_{(0,0)} \cap \mathfrak{c}_{\left(\varepsilon_{2}, 0\right)}=\left(c_{11}, c_{13} c_{31}\right)$,

(3) $\mathfrak{c}_{(0,1)} \cap \mathfrak{c}_{(0,0)}=\left(c_{23}, c_{11} c_{33}^{*}-c_{13} c_{31}\right)$.

Proof. As in the proof of Lemma 3.6.13, it suffices to work in the ring $\widetilde{R}$ which has the same presentation as $\bar{R}_{\overline{\mathfrak{M}}, \alpha}^{\operatorname{expl}, \nabla}$ except that all $c_{i j}^{*}$ are set to 1 . Recall that $\widetilde{c}_{32} \stackrel{\text { def }}{=} c_{32}-d_{22} c_{31}$ in $\widetilde{R}$.

We start with item (1). From Table (3), we easily deduce

$$
I_{\Lambda_{0}} \supseteq\left(c_{11}-c_{13} c_{31}, c_{23} c_{31}, c_{23} \widetilde{c}_{32}\right)
$$

that is, a surjection

$$
\widetilde{R} /\left(c_{11}-c_{13} c_{31}, c_{23} c_{31}, c_{23} \widetilde{c}_{32}\right) \rightarrow \widetilde{R} / I_{\Lambda_{0}}
$$


which we claim is an isomorphism. Note that, by construction, the ring $\widetilde{R} / I_{\Lambda_{0}}$ has three minimal primes, and it is equidimensional of dimension three.

From [LLHLM18, Proposition 8.11], we immediately deduce that the ring $\widetilde{R} /\left(c_{11}-c_{13} c_{31}, c_{23} c_{31}, c_{23} \widetilde{c}_{32}\right)$ is isomorphic to the quotient of the power series ring $\mathbb{F} \llbracket c_{31}, c_{13}, d_{22}, \widetilde{c}_{32}, c_{23} \rrbracket$ by the ideal generated by the following elements:

$$
\begin{aligned}
& c_{13} c_{31} c_{23}, \quad c_{13} c_{31} d_{22}-\frac{b-c}{a-b} c_{13} \widetilde{c}_{32}, \quad c_{23} c_{31}, \\
& c_{23} \widetilde{c}_{32}, \quad(a-b) c_{13} c_{31} d_{22}+(c-b) c_{13} \widetilde{c}_{32}+(-1-a+c) c_{23} c_{31}
\end{aligned}
$$

that is, by the ideal generated by

$$
c_{13}\left(c_{31} d_{22}-\frac{b-c}{a-b} \widetilde{c}_{32}\right), \quad c_{23} c_{31}, \quad c_{23} \widetilde{c}_{32},
$$

or, equivalently, the ideal generated by

$$
c_{13}\left(c_{31} d_{22}-\frac{b-c}{a-b} \widetilde{c}_{32}\right), \quad c_{23} c_{31}, \quad c_{23}\left(c_{31} d_{22}-\frac{b-c}{a-b} \widetilde{c}_{32}\right) .
$$

In other words, by an evident change of variables, we have

$$
\widetilde{R} /\left(c_{11}-c_{13} c_{31}, c_{23} c_{31}, c_{23} \widetilde{c}_{32}\right) \cong \mathbb{F} \llbracket X, Y, Z, W, c_{22}^{\prime} \rrbracket /(X Y, Y Z, W Z)
$$

and the latter ring is equidimensional of dimension three and has three irreducible components. It is, moreover, reduced since the ideal of relations is generated by square-free monomials.

The argument to prove (2) is completely analogous and we only sketch it. It is immediate to obtain from Table 3 the inclusion $\mathfrak{c}_{\left(\varepsilon_{1}, 1\right)} \cap \mathfrak{c}_{(0,0)} \cap \mathfrak{c}_{\left(\varepsilon_{2}, 0\right)} \supseteq\left(c_{11}, c_{13} c_{31}\right)$. From [LLHLM18, Proposition 8.11], we see that $\widetilde{R} /\left(c_{11}, c_{13} c_{31}\right)$ is isomorphic to

$$
\begin{gathered}
\mathbb{F} \llbracket c_{13}, c_{23}, c_{31}, \widetilde{c}_{32}, d_{22} \rrbracket /\left(c_{13} c_{31}, c_{13} \widetilde{c}_{32}, c_{31} c_{23} \widetilde{c}_{32}, c_{31}\left(\kappa c_{23}-c_{13} d_{22}\right)\right)= \\
=\mathbb{F} \llbracket c_{13}, c_{23}, c_{31}, \widetilde{c}_{32}, d_{22} \rrbracket /\left(c_{13} c_{31}, c_{13} \widetilde{c}_{32}, c_{31} c_{23}\right),
\end{gathered}
$$

for some $\kappa \in \mathbb{F}^{\times}$depending on $(a, b, c)$. The conclusion is obtained by an analogous argument to the previous case.

The proof of item (3) is similar and left to the reader. (Note that $\mathfrak{c}_{(0,1)} \cap$ $\mathfrak{c}_{(0,0)} \supseteq\left(c_{23}, c_{11}-c_{13} c_{31}\right)$ and use the explicit presentation of $\widetilde{R}$ given by [LLHLM18, Proposition 8.11] to check that $\widetilde{R} /\left(c_{23}, c_{11}-c_{13} c_{31}\right)$ is reduced with two irreducible components of dimension three.) 
LEMMA 3.6.16. In $\bar{R}_{\overline{\mathfrak{M}}, \alpha}^{\mathrm{expl}, \nabla}$, we have

$$
\begin{aligned}
& \left(I_{\Lambda_{0}} \cap \mathfrak{c}_{\left(\varepsilon_{1}, 1\right)}\right)+\left(I_{\Lambda_{0}} \cap \mathfrak{c}_{\left(\varepsilon_{2}, 1\right)} \cap \mathfrak{c}_{\left(\varepsilon_{2}-\varepsilon_{1}, 0\right)}\right)=I_{\Lambda_{0}}, \\
& \left(\mathfrak{c}_{\left(\varepsilon_{1}, 1\right)} \cap \mathfrak{c}_{(0,0)} \cap \mathfrak{c}_{\left(\varepsilon_{2}, 0\right)} \cap \mathfrak{c}_{(0,1)}\right)+\left(\mathfrak{c}_{\left(\varepsilon_{1}, 1\right)} \cap \mathfrak{c}_{(0,0)} \cap \mathfrak{c}_{\left(\varepsilon_{2}, 0\right)} \cap \mathfrak{c}_{\left(\varepsilon_{2}, 1\right)}\right) \\
& \quad=\mathfrak{c}_{\left(\varepsilon_{1}, 1\right)} \cap \mathfrak{c}_{(0,0)} \cap \mathfrak{c}_{\left(\varepsilon_{2}, 0\right)}, \\
& \left(\mathfrak{c}_{(0,1)} \cap \mathfrak{c}_{(0,0)} \cap \mathfrak{c}_{\left(\varepsilon_{2}, 0\right)}\right)+\left(\mathfrak{c}_{(0,1)} \cap \mathfrak{c}_{(0,0)} \cap \mathfrak{c}_{\left(\varepsilon_{2}-\varepsilon_{1}, 0\right)}\right)=\mathfrak{c}_{(0,1)} \cap \mathfrak{c}_{(0,0)} .
\end{aligned}
$$

Proof. We work in $\widetilde{R}$ and set $\widetilde{c}_{32} \stackrel{\text { def }}{=} c_{32}-d_{22} c_{31}$. An elementary check on the list of generators of the ideals $I_{\Lambda_{0}}, \mathfrak{c}_{\left(\varepsilon_{2}, 1\right)}, \mathfrak{c}_{\left(\varepsilon_{2}-\varepsilon_{1}, 0\right)}$, and $\mathfrak{c}_{\left(\varepsilon_{1}, 1\right)}$ shows that

$$
\left(I_{\Lambda_{0}} \cap \mathfrak{c}_{\left(\varepsilon_{1}, 1\right)}\right) \supseteq\left(c_{11}-c_{13} c_{31}, c_{23} c_{31}\right), \quad\left(I_{\Lambda_{0}} \cap \mathfrak{c}_{\left(\varepsilon_{2}, 1\right)} \cap \mathfrak{c}_{\left(\varepsilon_{2}-\varepsilon_{1}, 0\right)}\right) \supseteq\left(c_{23} \widetilde{c}_{32}\right),
$$

and we deduce (again by looking at the list of generators of $I_{\Lambda_{0}}$ ) that

$$
\left(I_{\Lambda_{0}} \cap \mathfrak{c}_{\left(\varepsilon_{1}, 1\right)}\right)+\left(I_{\Lambda_{0}} \cap \mathfrak{c}_{\left(\varepsilon_{2}, 1\right)} \cap \mathfrak{c}_{\left(\varepsilon_{2}-\varepsilon_{1}, 0\right)}\right) \supseteq I_{\Lambda_{0}} .
$$

The reverse inclusion is obvious.

Similarly, $\left(c_{11}\right) \subseteq \mathfrak{c}_{\left(\varepsilon_{1}, 1\right)} \cap \mathfrak{c}_{(0,0)} \cap \mathfrak{c}_{\left(\varepsilon_{2}, 0\right)} \cap \mathfrak{c}_{\left(\varepsilon_{2}, 1\right)}$ and $\left(c_{11}-c_{13} c_{31}\right) \subseteq \mathfrak{c}_{\left(\varepsilon_{1}, 1\right)} \cap \mathfrak{c}_{(0,0)} \cap$ $\mathfrak{c}_{\left(\varepsilon_{2}, 0\right)} \cap \mathfrak{c}_{(0,1)}$. Hence, $\left(\mathfrak{c}_{\left(\varepsilon_{1}, 1\right)} \cap \mathfrak{c}_{(0,0)} \cap \mathfrak{c}_{\left(\varepsilon_{2}, 0\right)} \cap \mathfrak{c}_{(0,1)}\right)+\left(\mathfrak{c}_{\left(\varepsilon_{1}, 1\right)} \cap \mathfrak{c}_{(0,0)} \cap \mathfrak{c}_{\left(\varepsilon_{2}, 0\right)} \cap \mathfrak{c}_{\left(\varepsilon_{2}, 1\right)}\right) \supseteq$ $\mathfrak{c}_{\left(\varepsilon_{1}, 1\right)} \cap \mathfrak{c}_{(0,0)} \cap \mathfrak{c}_{\left(\varepsilon_{2}, 0\right)}$.

The proof of item (3.19) is similar and left to the reader (note that $c_{11}-c_{13} c_{31} \in$ $\left(\mathfrak{c}_{(0,1)} \cap \mathfrak{c}_{(0,0)} \cap \mathfrak{c}_{\left(\varepsilon_{2}, 0\right)}\right)$ and $\left.c_{23} \in\left(\mathfrak{c}_{(0,1)} \cap \mathfrak{c}_{(0,0)} \cap \mathfrak{c}_{\left(\varepsilon_{2}-\varepsilon_{1}, 0\right)}\right)\right)$.

REMARK 3.6.17. The ideal relation appearing in Lemmas 3.6.15 and 3.6.16 are compatible with the outer automorphism of $\tilde{W}^{\vee}$. Explicitly, define $\tilde{\delta} \stackrel{\text { def }}{=}$ $(123) t_{(0,0,-1)}$ and let $\widetilde{w} \mapsto \tilde{\delta} \widetilde{w} \tilde{\delta}^{-1}$ be the corresponding outer automorphism on $\widetilde{W}^{\vee}$. Then $\delta$ acts on $\Sigma_{0}$ and the ideal relations of Lemmas 3.6.15 and 3.6.16 hold for shape $\beta$ with $\mathfrak{c}_{\left(\omega_{i}, a_{i}\right)}$ replaced by $\mathfrak{c}_{\left(\left(\widetilde{\delta}^{*}\right)^{-1}\left(\omega_{i}\right), a_{i}\right)}$. As an example, for shape $\beta$, the relation (3.19) becomes $\left(\mathfrak{c}_{\left(\varepsilon_{1}, 1\right)} \cap \mathfrak{c}_{\left(\varepsilon_{1}, 0\right)} \cap \mathfrak{c}_{(0,0)}\right)+\left(\mathfrak{c}_{\left(\varepsilon_{1}, 1\right)} \cap \mathfrak{c}_{\left(\varepsilon_{1}, 0\right)} \cap \mathfrak{c}_{\left(\varepsilon_{1}-\varepsilon_{2}, 0\right)}\right)=$ $\mathfrak{c}_{\left(\varepsilon_{1}, 1\right)} \cap \mathfrak{c}_{\left(\varepsilon_{1}, 0\right)}$.

\section{Lattices in generic Deligne-Lusztig representations}

The aim of this section is to classify lattices with irreducible cosocle in generic $\mathrm{GL}_{3}\left(\mathbb{F}_{q}\right)$ Deligne-Lusztig representations, providing the crucial representationtheoretic input to deduce Breuil's lattice conjecture from the weight part of Serre conjecture. The main result (Theorem 4.1.9) states that the submodule structure of lattices with irreducible cosocle can be predicted using the extension graph introduced in Section 2. 
Outline of the proof. Let $R$ be a generic Deligne-Lusztig representation. We have two main steps in the proof of the classification theorem: first, by local algebraic methods, we describe the reduction of lattices with irreducible cosocles isomorphic to a lower alcove weight with defect zero (Theorem 4.2.16). Second, in Section 4.3, we introduce another notion of distance related to the second part of Theorem 4.1.9, which we call saturation distance. It turns out that this notion is closely related to the submodule structure of the reduction of lattices.

Using a crucial global input coming from the geometry of Galois deformation rings, we show that

(1) if measured from $\sigma$ the graph and saturation distances coincide, then the reduction of the lattice with cosocle isomorphic to $\sigma$ is as predicted in Theorem 4.1.9 (Proposition 4.3.16);

(2) measured from a lower alcove weight of defect zero, the saturation distance and the graph distance coincide (Proposition 4.3.17, which uses Theorem 4.2.16).

In contrast to the other notions of distance that we introduce, the saturation distance involves lattices in characteristic zero, making it far more flexible. Taking advantage of this flexibility, we finally show by an induction on defect that the saturation distance and the graph distance coincide, completing the proof of Theorem 4.1.9.

Structure of Section 4. For $R$ as above and $\sigma \in \mathrm{JH}(\bar{R})$, let $R^{\sigma}$ denote the unique (up to homothety) $\mathcal{O}$-lattice in $R$ with irreducible cosocle $\sigma$ and write $\bar{R}^{\sigma}$ to denote its reduction modulo $\varpi$. The first main step is in Section 4.2. The argument uses the modular representation theory of algebraic groups to embed $\bar{R}^{\sigma}$ into the $\mathrm{G} \stackrel{\text { def }}{=} \underline{G}_{0}\left(\mathbb{F}_{p}\right)$-restriction of a tensor product $V_{\mu}$ of algebraic Weyl modules with non- $p$-restricted highest weight (see Section 4.2.2 for the definition of $V_{\mu}$ ). The content of Section 4.2.3 is the description of $\left.V_{\mu}\right|_{\mathrm{G}}$ provided by Theorem 4.2.7. This theorem describes the Jordan-Hölder constituents of $\left.V_{\mu}\right|_{\mathrm{G}}$ and the existence of nontrivial extensions between constituents at graph distance one (cf. the key technical result Proposition 4.2.10). The embedding of $\bar{R}^{\sigma}$ in $V_{\mu}$ is constructed in Section 4.2.4. One first proves the existence of a nonzero (and unique up to scalar) morphism $\bar{R}^{\sigma} \rightarrow V_{\mu}$ (Proposition 4.2.15); an inductive argument, using the description of the submodule structure of $\left.V_{\mu}\right|_{\mathrm{G}}$, then shows that the image of this morphism contains all the constituents of $\bar{R}^{\sigma}$. The submodule structure of $\bar{R}^{\sigma}$ is then obtained from that of $V_{\mu}$.

The second part of the proof of Theorem 4.1.9 is the content of Section 4.3. The key insight is the introduction of the auxiliary notion of saturation distance on $\mathrm{JH}(\bar{R})$ in Section 4.3.1 which relates the position of saturated lattices in $R^{\sigma}$. 
Using a global input, we give in Section 4.3.1 a first coarse relation between the saturation and the graph distance (cf. Corollary 4.3.8). Subsequently, we prove in Section 4.3.2 that the three distances are actually equal provided that $\sigma$ verifies an appropriate condition relating its defect, its graph distance, and its saturated distance (we say that $\sigma$ is maximally saturated in $R$ ). In particular, if $\sigma$ is maximally saturated in $R$, the structure of $\bar{R}^{\sigma}$ is predicted by the extension graph (Proposition 4.3.16).

We are hence left to prove that all constituents of $R$ are maximally saturated. This is shown by an induction argument in Section 4.3.3. The proof of Theorem 4.1.9 concludes this section.

\subsection{The classification statement.}

4.1.1. Some generalities. Let $\mathcal{C}$ be a nonzero finite abelian category over $\mathbb{F}$. Let $M$ be a nonzero object of $\mathcal{C}$. A decreasing (respectively increasing) filtration $\mathscr{F}$ on $M$ is a collection of subobjects $\mathscr{F}^{n}(M) \subset M$ (respectively $\mathscr{F}_{n}(M) \subset M$ ) for $n \in \mathbb{Z}$ such that $\mathscr{F}^{n+1}(M) \subset \mathscr{F}^{n}(M)$ (respectively $\mathscr{F}_{n}(M) \subset \mathscr{F}_{n+1}(M)$ ) for all $i$. A filtration $\mathscr{F}$ is exhaustive and separated if $\mathscr{F}^{n}(M)=0$ for $i$ sufficiently large (respectively small) and $\mathscr{F}^{n}(M)=M$ for $n$ sufficiently small (respectively large), and this property will always be assumed to hold. We write $\operatorname{gr}_{\mathscr{F}}^{n}(M) \stackrel{\text { def }}{=} \frac{\mathscr{F}^{n}(M)}{\mathscr{F}^{n+1}(M)}$ (respectively $\operatorname{gr}_{n}^{\mathscr{F}}(M) \stackrel{\text { def }}{=} \frac{\mathscr{F}_{n}(M)}{\mathscr{F}_{n-1}(M)}$ ) and omit $\mathscr{F}$ from the notation if it is clear from context. A filtration is semisimple if $\operatorname{gr}_{\mathscr{F}}^{n}(M)$ (respectively $\operatorname{gr}_{n}^{\mathscr{F}}(M)$ ) is semisimple for all $n \in \mathbb{Z}$. By shifting the filtration, we will assume that $\operatorname{gr}_{\mathscr{F}}^{n}(M)=0$ (respectively $\operatorname{gr}_{n}^{\mathscr{F}}(M)=0$ ) for $n<0$ and that $\operatorname{gr}_{\mathscr{F}}^{0}(M) \neq 0$ (respectively $\operatorname{gr}_{0}^{\mathscr{F}}(M) \neq 0$ ). The length of a filtration is the maximal $\ell \in \mathbb{Z}$ such that $\operatorname{gr}_{\mathscr{F}}^{\ell-1}(M) \neq 0$ (respectively $\operatorname{gr}_{\ell-1}^{\mathscr{F}}(M) \neq 0$ ).

The socle of $M$, denoted as $\operatorname{soc}(M)$, is defined to be the maximal (with respect to inclusion) semisimple subobject of $M$. The radical of $M$, denoted as $\operatorname{rad}(M)$, is the minimal (with respect to inclusion) subobject of $M$ whose corresponding quotient is semisimple. The cosocle of $M$ is $\operatorname{cosoc}(M) \stackrel{\text { def }}{=} M / \operatorname{rad}(M)$. We inductively define the radical and socle filtration on $M$ : we set $\operatorname{rad}^{0}(M)=M$ and let $\operatorname{rad}^{n}(M) \stackrel{\text { def }}{=} \operatorname{rad}\left(\operatorname{rad}^{n-1}(M)\right)$, and set $\operatorname{soc}_{-1}(M)=0$ and let $\operatorname{soc}_{n}(M)$ be the inverse image, via the canonical projection $M \rightarrow M / \operatorname{soc}_{n-1}(M)$, of $\operatorname{soc}\left(M / \operatorname{soc}_{n-1}(M)\right) \subseteq M / \operatorname{soc}_{n-1}(M)$. Then the radical (respectively socle) filtration is a decreasing (respectively increasing) semisimple filtration. Moreover, $\operatorname{gr}_{\mathrm{rad}}^{0}(M)=\operatorname{cosoc}(M)$ and $\operatorname{gr}_{0}^{\mathrm{soc}}(M)=\operatorname{soc}(M)$. Since formation of cosocle (respectively socle) is right (respectively left) exact, the filtration induced from the radical (respectively socle) filtration on a quotient object (respectively subobject) is the radical (respectively socle) filtration. The lengths 
of the radical and socle filtrations coincide, and we call this value the Loewy length of $M$ and denote it by $\ell \ell(M)$. Any semisimple filtration has length at least $\ell \ell(M)$, and we say that it is a Loewy series if its length equals $\ell \ell(M)$. If $\mathscr{F}$ is a decreasing Loewy series, then we necessarily have

$$
\operatorname{rad}^{n}(M) \subseteq \mathscr{F}^{n}(M) \subseteq \operatorname{soc}_{\ell \ell(M)-n-1}(M)
$$

for all $n \in\{0, \ldots, \ell \ell(M)\}$. We say that $M$ is rigid if $\operatorname{rad}^{n}(M)=\operatorname{soc}_{\ell \ell(M)-n-1}(M)$ for all $n$.

We say that $M$ is multiplicity free if every Jordan-Hölder factor of $M$ appears with multiplicity one. We now suppose that $M$ is multiplicity free. We say that $\sigma \in \mathrm{JH}(M)$ points to $\sigma^{\prime} \in \mathrm{JH}(M)$ if there exists a subquotient of $M$ which is isomorphic to a nontrivial extension of $\sigma$ by $\sigma^{\prime}$. We say that a subset $S \subset \mathrm{JH}(M)$ is closed if $\sigma \in S$ and $\sigma$ points to $\sigma^{\prime}$ imply that $\sigma^{\prime} \in S$.

PROPOSITION 4.1.1. The assignment of $\mathrm{JH}(N)$ to a subobject $N \subset M$ gives a bijection between subobjects of $M$ and closed subsets of $\mathrm{JH}(M)$.

Proof. It is easy to see that $\mathrm{JH}(N)$ is a closed subset of $\mathrm{JH}(M)$. Suppose that $S \subset$ $\mathrm{JH}(M)$ is a closed subset. Let $N$ be the minimal subobject of $M$ with $S \subset \mathrm{JH}(N)$. Suppose that $\mathrm{JH}(N) \backslash S$ is nonempty and contains $\sigma$. If $\sigma^{\prime} \in \mathrm{JH}(N)$ points to $\sigma$, then $\sigma^{\prime}$ is not in $S$ since $S$ is closed. By replacing $\sigma$ by $\sigma^{\prime}$ repeatedly, we can assume, without loss of generality, that $\sigma^{\prime}$ does not point to $\sigma$ for all $\sigma^{\prime} \in \mathrm{JH}(N)$. Let $N^{\prime}$ be the maximal subobject of $N$ such that $\sigma \notin \mathrm{JH}\left(N^{\prime}\right)$. This maximality implies that the socle of $N / N^{\prime}$ must be isomorphic to $\sigma$. By the assumption above, there is no subobject of $N / N^{\prime}$ which is an extension by $\sigma$, and, therefore, $N / N^{\prime}$ is isomorphic to $\sigma$. Then the existence of $N^{\prime}$ contradicts the minimality of $N$.

Suppose that $M$ is multiplicity free and that $\mathscr{F}$ is a decreasing (respectively increasing) filtration on $M$. For $\sigma \in \mathrm{JH}(M)$, we define $\mathrm{d}_{\mathscr{F}}^{M}(\sigma)$ (respectively $\left.\mathrm{d}_{M}^{\mathscr{F}}(\sigma)\right)$ to be the unique value $n$ such that $\operatorname{Hom}_{\mathcal{C}}\left(\sigma, \operatorname{gr}_{\mathscr{F}}^{n}(M)\right.$ ) (respectively $\left.\operatorname{Hom}_{\mathcal{C}}\left(\sigma, \operatorname{gr}_{n}^{\mathscr{F}}(M)\right)\right)$ is nonzero. For $\mathscr{F}$ semisimple, we say that $\sigma \in \mathrm{JH}(M)$ $\mathscr{F}$-points to $\sigma^{\prime} \in \mathrm{JH}(M)$ if $\sigma$ points to $\sigma^{\prime}$ and the (shifted) induced filtration on the subquotient which is isomorphic to a nontrivial extension of $\sigma$ by $\sigma^{\prime}$ has length 2.

If $M$ is multiplicity free, we can attach a directed acyclic graph $\Gamma(M)$ and a subgraph $\Gamma_{\mathscr{F}}(M)$ of $\Gamma(M)$ (respectively $\Gamma^{\mathscr{F}}(M)$ ) of $\Gamma(M)$ ) where the vertices are in bijection with the Jordan-Hölder factors of $M$ and there is an arrow $\sigma \rightarrow$ $\sigma^{\prime}$ in $\Gamma(M)$ if $\sigma$ points to $\sigma^{\prime}$ and an arrow $\sigma \rightarrow \sigma^{\prime}$ in $\Gamma_{\mathscr{F}}(M)$ (respectively in $\Gamma^{\mathscr{F}}(M)$ ) if $\sigma \mathscr{F}$-points to $\sigma^{\prime}$. An extension path (in $M$ ) is a directed path in 
$\Gamma(M)$ and an extension path in $\mathscr{F}$ is a directed path in $\Gamma_{\mathscr{F}}(M)$ (respectively $\left.\Gamma^{\mathscr{F}}(M)\right)$. The following proposition is immediate from the definitions.

PROPOSITION 4.1.2. Let $N$ be a subquotient of $M$. If $\mathscr{F}$ is a semisimple filtration on $M$, then it naturally induces a semisimple filtration on $N$. Moreover, $\Gamma(N)$ (respectively $\Gamma_{\mathscr{F}}(N)$ and $\Gamma^{\mathscr{F}}(N)$ ) is the maximal subgraph of $\Gamma(M)$ (respectively $\Gamma_{\mathscr{F}}(M)$ and $\Gamma^{\mathscr{F}}(M)$ ) with vertices corresponding to $\mathrm{JH}(N)$.

LEMMA 4.1.3. Suppose that $M$ is multiplicity free and $\sigma \in \mathrm{JH}(M)$. If $\mathrm{d}_{\mathrm{rad}}^{M}(\sigma)>$ 0 (respectively $\mathrm{d}_{M}^{\mathrm{soc}}(\sigma)>0$ ), there is a $\sigma^{\prime}$ such that $\mathrm{d}_{\mathrm{rad}}^{M}\left(\sigma^{\prime}\right)=\mathrm{d}_{\mathrm{rad}}^{M}(\sigma)-1$ (respectively $\mathrm{d}_{M}^{\mathrm{soc}}\left(\sigma^{\prime}\right)=\mathrm{d}_{M}^{\mathrm{soc}}(\sigma)-1$ ) and $\sigma^{\prime}$ rad-points to $\sigma$ (respectively $\sigma$ socpoints to $\left.\sigma^{\prime}\right)$.

Proof. We consider the radical filtration; the socle filtration is analyzed similarly. Let $d=\mathrm{d}_{\mathrm{rad}}^{M}(\sigma)$. Then the radical filtration on $\operatorname{rad}^{d-1}(M)$ is a shift of the radical filtration on $M$ by definition and induces the radical filtration on $\operatorname{rad}^{d-1}(M) / \operatorname{rad}^{d+1}(M)$. Then there is a $\sigma^{\prime} \in \mathrm{JH}\left(\mathrm{gr}_{\mathrm{rad}}^{d-1}(M)\right)$ which rad-points to $\sigma$, otherwise $\sigma$ would not be in the radical of $\operatorname{rad}^{d-1}(M) / \operatorname{rad}^{d+1}(M)$ which is $\operatorname{gr}_{\text {rad }}^{d}(M)$.

Corollary 4.1.4. Suppose that $M$ is multiplicity free and $\sigma \in \mathrm{JH}(M)$. Then there is an extension path in the radical (respectively socle) filtration of length $\mathrm{d}_{\mathrm{rad}}^{M}(\sigma)$ (respectively $\mathrm{d}_{M}^{\mathrm{soc}}(\sigma)$ ) ending (respectively beginning) with $\sigma$.

Proof. The case $\mathrm{d}_{\mathrm{rad}}^{M}(\sigma)=0$ (respectively $\mathrm{d}_{M}^{\mathrm{soc}}(\sigma)=0$ ) is trivial. The induction step follows from Lemma 4.1.3.

Lemma 4.1.5. Suppose that $M$ is multiplicity free and $\mathscr{F}$ is an increasing semisimple filtration on $M$. If $\sigma$ points to $\sigma^{\prime}$, then $d_{M}^{\mathscr{F}}(\sigma)>d_{M}^{\mathscr{F}}\left(\sigma^{\prime}\right)$.

Proof. Let $d$ be $d_{M}^{\mathscr{F}}(\sigma)$. By Proposition 4.1.1, $\mathscr{F}_{d}(M)$ contains $\sigma^{\prime}$ as a JordanHölder factor. Thus, $d_{M}^{\mathscr{F}}(\sigma) \geqslant d_{M}^{\mathscr{F}}\left(\sigma^{\prime}\right)$. If $d_{M}^{\mathscr{F}}(\sigma)=d_{M}^{\mathscr{F}}\left(\sigma^{\prime}\right)$, then there is a subquotient of $M$ which is isomorphic to a direct sum of $\sigma$ and $\sigma^{\prime}$. This contradicts the fact that $\sigma$ points to $\sigma^{\prime}$.

Proposition 4.1.6. Suppose that $M \in \mathcal{C}$ is multiplicity free. Then $M$ is rigid if and only if for every $\sigma \in \mathrm{JH}(M)$, there is an extension path in the radical (respectively socle) filtration of length $\ell \ell(M)-1-\mathrm{d}_{\mathrm{rad}}^{M}(\sigma)$ (respectively $\ell \ell(M)-$ $\left.1-\mathrm{d}_{M}^{\mathrm{soc}}(\sigma)\right)$ beginning (respectively ending) with $\sigma$. 
Proof. First, suppose that $M$ is rigid. There is an extension path $P_{\text {rad }}$ in the radical filtration of length $\mathrm{d}_{\mathrm{rad}}^{M}(\sigma)$ ending at $\sigma$ by Corollary 4.1.4. By rigidity, this is an extension path in the socle filtration of length $\mathrm{d}_{\mathrm{rad}}^{M}(\sigma)=\ell \ell(M)-1-\mathrm{d}_{M}^{\mathrm{soc}}(\sigma)$.

Now suppose that there is an extension path in the radical filtration of length $\ell \ell(M)-1-\mathrm{d}_{\mathrm{rad}}^{M}(\sigma)$ starting with $\sigma$. Then

$$
\mathrm{d}_{M}^{\mathrm{soc}}(\sigma) \geqslant \ell \ell(M)-1-\mathrm{d}_{\mathrm{rad}}^{M}(\sigma)
$$

by Lemma 4.1.5. The reverse inequality is implied by (4.1), and we conclude that $\operatorname{gr}_{\mathrm{rad}}^{n}(M)$ and $\operatorname{gr}_{\ell \ell(M)-1-n}^{\mathrm{soc}}(M)$ are isomorphic and, thus, that $M$ is rigid.

The following is self-evident.

Proposition 4.1.7. Let $M \in \mathcal{C}$ be multiplicity free. Then the dual object $M^{*}$ in the dual abelian category $\mathcal{C}^{*}$ is also multiplicity free. A decreasing filtration $\mathscr{F}$ on $M$ gives rise to an increasing filtration $\mathscr{F}^{*}$ on $M^{*}$. Then the map sending $\sigma \in$ $\mathrm{JH}(M)$ to $\sigma^{*} \in \mathrm{JH}\left(M^{*}\right)$ extends to isomorphisms of directed graphs $\Gamma(M) \stackrel{\sim}{\rightarrow}$ $\Gamma\left(M^{*}\right)$ and $\Gamma_{\mathscr{F}}(M) \stackrel{\sim}{\rightarrow} \Gamma^{\mathscr{F} *}(M)^{*}$ where $-{ }^{*}$ denotes the transpose of a directed graph. In particular, if $\sigma_{0} \rightarrow \sigma_{1} \rightarrow \cdots \rightarrow \sigma_{n}$ is an extension path in $\mathscr{F}$, then $\sigma_{n}^{*} \rightarrow \sigma_{n-1}^{*} \rightarrow \cdots \rightarrow \sigma_{0}^{*}$ is an extension path in $\mathscr{F}^{*}$.

4.1.2. The main result. We now use the notation from Section 4.1 .1 with $\mathcal{C}$ the category of finite $\mathbb{F}[\mathrm{G}]$-modules. Let $R$ be a 2-generic Deligne-Lusztig representation of G over E. By [Her09, Appendix, Theorem 3.4] (see the proof of Proposition 2.3.5), $R$ is residually multiplicity free. We will show that the elements in $\mathrm{JH}(\bar{R})$ are $p$-regular in Lemma 4.2.13. If $\sigma \in \mathrm{JH}(\bar{R})$, then there exists a unique (up to homothety) $\mathcal{O}$-lattice $R^{\sigma} \subseteq R$ with irreducible cosocle isomorphic to $\sigma$ by [EGS15, Lemma 4.1.1], and we write $\bar{R}^{\sigma}$ to denote its reduction modulo $\varpi$. In Section 2, we defined a distance function $d_{\text {gph }}$ on $p$ regular Serre weights. We now want to relate the graph distance to the submodule structure of $\bar{R}^{\sigma}$. To simplify notation, we fix $R$ and write $\mathrm{d}_{\text {rad }}^{\sigma}\left(\sigma^{\prime}\right)$ for $\mathrm{d}_{\text {rad }}^{\bar{R}^{\sigma}}\left(\sigma^{\prime}\right)$.

Definition 4.1.8. Let $V$ be a set of (isomorphism classes of) weights $\sigma=$ $F(\mu)$ with $\mu p$-regular. Let $\sigma \in V$. Let $\Gamma$ be a directed graph with vertex set $V$. Then we say that $\Gamma$ is predicted by the extension graph with respect to $\sigma$ if there is an edge from $\kappa_{1}$ to $\kappa_{2}$ if and only if $\mathrm{d}_{\mathrm{gph}}\left(\kappa_{1}, \kappa_{2}\right)=1$ and $\mathrm{d}_{\mathrm{gph}}\left(\sigma, \kappa_{1}\right) \leqslant \mathrm{d}_{\mathrm{gph}}(\sigma$, $\kappa_{2}$ ) (see Definition 2.1.8).

Our main result on the representation theory side is the following.

THEOREM 4.1.9. Let $R$ be as above and 13-generic. Let $\sigma \in \mathrm{JH}(\bar{R})$. Then 
(1) $\mathrm{d}_{\mathrm{gph}}(\sigma, \kappa)=\mathrm{d}_{\mathrm{rad}}^{\sigma}(\kappa)$ for all $\kappa \in \mathrm{JH}(\bar{R})$, in particular,

$$
\ell \ell\left(\bar{R}^{\sigma}\right)=2 \operatorname{Def}_{R}(\sigma)+3\left(f-\operatorname{Def}_{R}(\sigma)\right)+1 ;
$$

(2) $\Gamma_{\text {rad }}\left(\bar{R}^{\sigma}\right)$ is predicted by the extension graph with respect to $\sigma$;

(3) $\Gamma\left(\bar{R}^{\sigma}\right)$ is predicted by the extension graph with respect to $\sigma$;

(4) $\bar{R}^{\sigma}$ is rigid; and

(5) if $\kappa \in \mathrm{JH}(\bar{R})$ and $R^{\kappa} \hookrightarrow R^{\sigma}$ is a saturated inclusion, then $p^{\mathrm{d}_{\operatorname{gph}}(\kappa, \sigma)} R^{\sigma} \hookrightarrow$ $R^{\kappa}$ is a saturated inclusion.

REMARK 4.1.10. By Proposition 2.3.5, every maximal geodesic in $\mathrm{JH}(\bar{R})$ starting from $\sigma$ has the same length. Then item (4) follows from items (1) and (2) and Proposition 4.1.6. Furthermore, (3) and Proposition 4.1.1 give a classification of submodules of $\bar{R}^{\sigma}$, from which one can easily deduce items (1) and (2).

The proof of Theorem 4.1 .9 will be carried out in the following subsections.

4.2. Injective envelopes. We now relax our hypotheses on $\underline{G}_{0}$ but keep much of the related notation. Let $\underline{G}_{0}$ be a connected reductive group over $\mathbb{F}_{p}$ and let $\underline{G}$ be the base change $\underline{G}_{0} \times_{\mathbb{F}_{p}} \mathbb{F}$. Assume that $\underline{G}$ is split and isomorphic to $\underline{G}_{s} \times_{\mathbb{F}_{p}} \mathbb{F}$, where $\underline{G}_{s}$ is a connected split reductive group over $\mathbb{F}_{p}$. Let $\underline{G}_{0}^{\text {der }}$ be the derived subgroup of $\underline{G}_{0}$ and let $\underline{G}^{\text {der }}$ be the base change $\underline{G}_{0}^{\text {der }} \times_{\mathbb{F}_{p}} \mathbb{F}$. Assume that $\underline{G}_{0}^{\text {der }}$ is simply connected. Let $\mathrm{G}$ (respectively $\mathrm{G}^{\mathrm{der}}$ ) be the finite group $\underline{G}_{0}\left(\mathbb{F}_{p}\right)$ (respectively $\underline{G}_{0}^{\text {der }}\left(\mathbb{F}_{p}\right)$ ). Let $F: \underline{G} \rightarrow \underline{G}$ denote the relative Frobenius with respect to $\underline{G}_{s}$. There is an automorphism $\pi$ of $\underline{G}_{s}$, and hence its based root datum, so that $F \circ \pi: \underline{G} \rightarrow \underline{G}$ is the relative Frobenius with respect to $\underline{G}_{0}$. This definition of $\pi$ is consistent with the special case introduced in Section 1.4. Let $h$ be the Coxeter number of $\underline{G}$. We will eventually specialize to the case where $\underline{G}$ is a product of copies of $\mathrm{GL}_{3}$ so that $h=3$.

We define $\operatorname{Proj}(\sigma)$ to be the projective hull of $\sigma$ in the category of $\mathbb{F}[\mathrm{G}]$ modules. As $\mathbb{F}[\mathrm{G}]$ is a Frobenius algebra, we have an isomorphism $\operatorname{Proj}(\sigma) \cong$ $\operatorname{Inj}(\sigma)$ where $\operatorname{Inj}(\sigma)$ denotes the injective envelope of $\sigma$ (again in the category of $\mathbb{F}[\mathrm{G}]$-modules; cf. [Alp86, Section 6, Theorems 4 and 6]).

4.2.1. Algebraic groups, Frobenius kernels, and finite groups. In this section, we compare injective envelopes of weights for representations of Frobenius kernel and of finite groups. One goal is to prove that, under genericity conditions, 
the graph distance introduced in Section 2.1 can be characterized in terms of nonvanishing of $\mathrm{Ext}_{\mathrm{G}}^{1}$-groups (Lemma 4.2.6). We, moreover, introduce and describe a non- $p$-restricted Weyl module $V_{\mu}$ which will play a key role in the proof of a particular case of Theorem 4.1.9.

Let $\underline{G}_{1}$ denote the Frobenius kernel of $\underline{G}$ and $\underline{G}_{1} \underline{T}$ denote the product of $\underline{T}$ and $\underline{G}_{1}$ in $\underline{G}$. We similarly define $\underline{G}_{1}^{\text {der }}$ and $\underline{G}_{1}^{\text {der }} \underline{T}^{\text {der }}$. The $\underline{G}$-representation $L(\mu)$ remains irreducible when restricted to $\underline{G}_{1} \underline{T}$ and $\underline{G}_{1}$ (cf. [Jan03, II.3.10 and II.9.6]) and we will use the standard notations $L_{1}(\mu)=\left.L(\mu)\right|_{\underline{G}_{1}}, \widehat{L}_{1}(\mu)=$ $\left.L(\mu)\right|_{\underline{G}_{1} \underline{T}}$ (cf. loc. cit.). We write $\widehat{Q}_{1}(\mu)$ to denote the injective envelope of the irreducible representation $\widehat{L}_{1}(\mu)$ in the category of $\underline{G}_{1} \underline{T}$-modules. It restricts to an injective envelope of $L_{1}(\mu)$ in the category of $\underline{G}_{1}$-modules. As in the case of the finite group $\mathrm{G}$, it is isomorphic to a projective cover of $L_{1}(\mu)$ as well. We recall the following important result.

THEOREM 4.2.1. Assume that $p>2(h-1)$ and that $\underline{G}$ has no factors of type $A_{1}$. Then $\widehat{Q}_{1}(\mu)$ has a unique $\underline{G}$-module structure which will be denoted by $Q_{1}(\mu)$ in what follows. In particular, $\operatorname{soc}_{\underline{G}} Q_{1}(\mu)$ is isomorphic to $L(\mu)$ since $L(\mu)$ is the unique extension of $\widehat{L}_{1}(\mu)$. Assume that $\mu \in X_{1}(\underline{T)}$ is $h-2$-deep. Then

$$
\left.Q_{1}(\mu)\right|_{G} \cong \operatorname{Proj}(F(\mu)) \cong \operatorname{Inj}(F(\mu)) .
$$

Proof. The first part of the theorem is well known; cf. [Jan03, II.11.11]. In what follows, we deduce the isomorphism (4.2) from [Pil93, Lemma 6.1] (where it is stated when $\underline{G}$ is semisimple). It suffices to show that $\left.Q_{1}(\mu)\right|_{\mathrm{G}}$ is injective and its socle is $F(\mu)$.

Claim 1. Let $M$ be a $\underline{G}$-module. Then $\operatorname{soc}_{\underline{G}^{\text {der }}}\left(\left.M\right|_{\underline{G}^{\text {der }}}\right)=\left.\operatorname{soc}_{\underline{G}}(M)\right|_{\underline{G}^{\text {der }}}$. The analogous statement holds true for $\underline{G}_{1} \underline{T}$ and $\underline{G}_{1}^{\text {der }} \underline{T}^{\text {der }}$ and for the finite groups $\mathrm{G}$ and $\mathrm{G}^{\mathrm{der}}$.

Proof of Claim 1. Let $\underline{Z} \stackrel{\text { def }}{=} \underline{G} / \underline{G}^{\text {der }}$ (respectively $\underline{Z}_{1} \stackrel{\text { def }}{=} \underline{G}_{1} / \underline{G}_{1}^{\text {der }}$ ). For $\bullet \in$ $\{\varnothing, 1\}$, the group $\underline{Z}$. is diagonalizable, and, hence, by the Hochschild-Serre spectral sequence [Jan03, I.6.9(3)], the restriction functor Res $\underline{G}_{G_{0}^{\text {der }}}$ (which is exact) induces a canonical isomorphism

$$
\operatorname{Ext}_{\underline{G}_{\bullet}^{i}}^{i}(M, N) \stackrel{\sim}{\longrightarrow} H^{0}\left(\underline{Z}_{\bullet}, \operatorname{Ext}_{\underline{G}_{\bullet}^{\text {der }}}^{i}\left(\left.M\right|_{\underline{G}_{\bullet}^{\text {der }}},\left.N\right|_{\underline{G}_{\bullet}^{\text {der }}}\right)\right)
$$

for all $i \in \mathbb{N}$ and all $\underline{G}_{\text {.-modules }} M, N$. Since the $\underline{G}_{\text {- }}^{\text {der }}$-restriction of an irreducible $\underline{G}$.-module remains irreducible, we conclude that $\operatorname{Res}{ }_{G_{0}^{\text {der }}}$. commutes with the formation of socles and cosocles. By [Jan03, II.9.6 (11)], this implies the required statement for the groups $\underline{G}_{1} \underline{T}, \underline{G}_{1}^{\mathrm{der}} \underline{T}^{\mathrm{der}}$. 
Since $Z \stackrel{\text { def }}{=} \mathrm{G} / \mathrm{G}^{\mathrm{der}}$ has order prime to $p$, we also have a canonical isomorphism

$$
\operatorname{Ext}_{\mathrm{G}}^{i}(M, N) \stackrel{\sim}{\longrightarrow} H^{0}\left(\mathrm{Z}, \operatorname{Ext}_{\mathrm{G}^{\text {der }}}^{i}\left(\left.M\right|_{\mathrm{G}^{\mathrm{der}}},\left.N\right|_{\mathrm{G}^{\mathrm{der}}}\right)\right.
$$

for all $i \in \mathbb{N}$ and all G-modules $M, N$ which implies the statement in the case of finite groups.

Claim 2. Let $v$ be a $p$-restricted weight. Then $\left.\widehat{Q}_{1}(v)\right|_{\underline{G}_{1}^{\text {der }} \underline{T}^{\text {der }}}$ is the injective envelope of $\left.\widehat{L}_{1}(\nu)\right|_{\underline{G}_{1}^{\mathrm{der}}} \underline{T}^{\mathrm{der}}$ as a $\underline{G}_{1}^{\mathrm{der}} \underline{T}^{\mathrm{der}}$-module.

Proof of Claim 2. By Claim 1, the socle of $\left.\widehat{Q}_{1}(v)\right|_{G_{1}^{\text {der }}} \underline{T}^{\text {der }}$ is isomorphic to $\left.\widehat{L}_{1}(v)\right|_{\underline{G}_{1}^{\text {der }} \underline{T}^{\text {der }}}$. It suffices to prove injectivity. By [Jan03, II.9.4], it is enough to prove injectivity for the restriction to $\underline{G}_{1}^{\mathrm{der}}$. As $\underline{G}_{1}^{\mathrm{der}}$ and $\underline{G}_{1}$ are both finite and $\underline{G}_{1}^{\text {der }}$ is closed in $\underline{G}_{1}$, we deduce that $\operatorname{Res} \underline{G}_{\underline{G}_{1}^{\text {der }}}$ maps injectives to injectives (since it has an exact left adjoint, cf. [Jan03, I.3.5, I.8.16]).

We are now ready to prove (4.2). We show that $\left.Q_{1}(\mu)\right|_{\mathrm{G}}$ is an injective Gmodule with socle isomorphic to $F(\mu)$. By [Pil93, Lemma 6.1] (which also holds in the nonsplit case; cf. the final remark of [Pil93, Section 11]) and Claim 2, we have

$$
\left.Q_{1}(\mu)\right|_{\mathrm{G}^{\mathrm{der}}} \cong \operatorname{Inj}\left(\left.F(\mu)\right|_{\mathrm{G}^{\mathrm{der}}}\right)
$$

so that $\left.Q_{1}(\mu)\right|_{\mathrm{G}^{\mathrm{der}}}$ is an injective $\mathrm{G}^{\mathrm{der}}$-module with socle isomorphic to $\left.F(\mu)\right|_{\mathrm{G}^{\mathrm{der}}}$.

We first show injectivity. The functor of $\mathrm{G} / \mathrm{G}^{\mathrm{der}}$-invariants is exact on the category of $\mathrm{G} / \mathrm{G}^{\mathrm{der}}$-representations. We hence obtain a canonical isomorphism

$$
\operatorname{Hom}_{\mathrm{G}}\left(\bullet,\left.Q_{1}(\mu)\right|_{\mathrm{G}}\right) \cong\left(\operatorname{Hom}_{\mathrm{G}^{\prime}}\left(\bullet,\left.Q_{1}(\mu)\right|_{\mathrm{G}^{\mathrm{der}}}\right)\right)^{\mathrm{G} / \mathrm{G}^{\mathrm{der}}},
$$

and $\operatorname{Hom}_{\mathrm{G}}\left(\bullet,\left.Q_{1}(\mu)\right|_{\mathrm{G}}\right)$, being the composite of two exact functors, is therefore exact.

The socle of $\left.Q_{1}(\mu)\right|_{\mathrm{G}}$ contains a submodule isomorphic to $F(\mu)$ and its restriction to $\mathrm{G}^{\mathrm{der}}$ is isomorphic to $\left.F(\mu)\right|_{\mathrm{G}^{\mathrm{der}}}$. Thus, the socle of $\left.Q_{1}(\mu)\right|_{\mathrm{G}}$ is isomorphic to $F(\mu)$. (We are grateful to the referee for simplifying the argument in our first version.)

Recall that an irreducible $\underline{G}$-module $L(\kappa)$, with $\kappa \in X_{+}^{*}(\underline{T})$, is said to be $p$ bounded if $\left\langle\kappa, \alpha^{\vee}\right\rangle<2(h-1) p$ for all coroots $\alpha \in \underline{R}^{\vee}$; a $\underline{G}$-module is $p$-bounded if all its Jordan-Hölder factors are $p$-bounded. Similarly, a $\underline{G}$-module is defined to be $m$-deep if the highest weights of all its Jordan-Hölder factors are $m$-deep. The following lemmas will be used several times in the rest of this section. 
Lemma 4.2.2 [Pil97, Lemma 3.1]. Let $M$ be a $\underline{G}$-module. If $M$ is 3(h-1)-deep with p-bounded highest weight, then

$$
\left.\operatorname{soc}_{i}^{\underline{G}}(M)\right|_{\mathrm{G}}=\operatorname{soc}_{i}^{\mathrm{G}}\left(\left.M\right|_{\mathrm{G}}\right),\left.\quad \operatorname{rad}_{\underline{G}}^{i}(M)\right|_{\mathrm{G}}=\operatorname{rad}_{\mathrm{G}}^{i}\left(\left.M\right|_{\mathrm{G}}\right) .
$$

Proof. The statement on the socle filtration for $\underline{G}^{\text {der }}$ and $G^{\text {der }}$ follows from the proof of [Pil97, Lemma 3.1]. While loc. cit. assumes that $\underline{G}_{0}^{\text {der }}$ is split over $\mathbb{F}_{p}$, the proof applies setting $n=1$ and using that $\left.L\left(p \lambda_{1}\right)\right|_{\mathrm{G}^{\text {der }}}$ is isomorphic to $\left.L\left(\pi \lambda_{1}\right)\right|_{\mathrm{G}^{\text {der }}}$ rather than $\left.L\left(\lambda_{1}\right)\right|_{\mathrm{G}^{\text {der }}}$. By duality, noting that $M$ is $3(h-1)$-deep if and only if its linear dual $M^{*}$ is $3(h-1)$-deep and that $\operatorname{soc}_{\ell \ell(M)-i}^{\bullet}\left(M^{*}\right)=$ $\left(M / \operatorname{rad}_{\bullet}^{i}(M)\right)^{*}$ for $\bullet \in\left\{\mathrm{G}^{\mathrm{der}}, \underline{G}^{\mathrm{der}}\right\}$, we obtain the analogous statement for the radical filtration (recall that $\ell \ell(M)$ is the Loewy length of $M$ ). The general case follows from Claim 1 in the proof of Theorem 4.2.1.

Corollary 4.2.3. Let $\mu \in X_{1}(\underline{T})$ such that $Q_{1}(\mu)$ is 3(h-1)-deep. Then

$$
\left.\operatorname{soc}_{i}^{\underline{G}}\left(Q_{1}(\mu)\right)\right|_{\mathrm{G}}=\operatorname{soc}_{i}^{\mathrm{G}}(\operatorname{Inj}(F(\mu))),\left.\quad \operatorname{rad}_{\underline{G}}^{i}\left(Q_{1}(\mu)\right)\right|_{\mathrm{G}}=\operatorname{rad}_{\mathrm{G}}^{i}(\operatorname{Inj}(F(\mu))) .
$$

Proof. This follows from Theorem 4.2.1 and Lemma 4.2.2.

If $v, \kappa \in X^{*}(\underline{T})$, we let $m_{\kappa}(v) \stackrel{\text { def }}{=} \operatorname{dim}_{\mathbb{F}}(L(\kappa))_{\nu}$. Moreover, we write $v \in L(\kappa)$ as a shorthand for $(L(\kappa))_{v} \neq 0$.

Lemma 4.2.4 (Translation principle). Assume that $p \geqslant 2(h-1)$. Let $\lambda, \xi \in$ $X_{1}(\underline{T})$. Assume that for all weights $v \in L(\xi)$, the weights $\lambda+v$ belong to the same alcove as $\lambda$. Then we have the following isomorphism of $\underline{G}$-modules:

(1) $L(\lambda) \otimes_{\mathbb{F}} L(\xi)=\bigoplus_{v \in L(\xi)} L(\lambda+v)^{\oplus m_{\xi}(v)}$,

(2) $Q_{1}(\lambda) \otimes_{\mathbb{F}} L(\xi)=\bigoplus_{\nu \in L(\xi)} Q_{1}(\lambda+v)^{\oplus m_{\xi}(v)}$.

Proof. We first prove item (1). The isomorphism holds upon restriction to $G_{1}^{\text {der }}$ by [Pil93, Lemma 5.1]. As the LHS and RHS of (1) have the same central characters, the $\underline{G}_{1}^{\text {der }}$-isomorphisms extend to $\underline{G}$-isomorphisms by (4.3).

We now switch to item (2). By the same argument as above, we deduce from [Pil93, Lemma 5.1] a $\underline{G}_{1} \underline{T}$-equivariant isomorphism

$$
\widehat{Q}_{1}(\lambda) \otimes_{\mathbb{F}} \widehat{L}_{1}(\xi) \cong \bigoplus_{\nu \in \widehat{L}_{1}(\xi)} \widehat{Q}_{1}(\lambda+v)^{\oplus m_{\xi}(\nu)} .
$$


By item (1) and the isomorphism $\operatorname{soc}_{G}\left(Q_{1}(\lambda)\right) \cong L(\lambda)$ from Theorem 4.2.1, we have a $\underline{G}$-equivariant injection

$$
\begin{aligned}
\operatorname{soc}_{\underline{G}}\left(\bigoplus_{\nu \in L(\xi)} Q_{1}(\lambda+v)^{\oplus m_{\xi}(v)}\right) & =\bigoplus_{\nu \in L(\xi)} L(\lambda+v)^{\oplus m_{\xi}(v)} \\
& =L(\lambda) \otimes_{\mathbb{F}} L(\xi) \hookrightarrow Q_{1}(\lambda) \otimes_{\mathbb{F}} L(\xi) .
\end{aligned}
$$

We claim that in the full subcategory of $p$-bounded $\underline{G}$-modules, the functor $\operatorname{Hom}_{\underline{G}}\left(\bullet, Q_{1}(\lambda) \otimes_{\mathbb{F}} L(\xi)\right)$ is exact. Granting the claim we deduce from (4.7) a $\underline{G}$-equivariant morphism

$$
\bigoplus_{v \in L(\xi)} Q_{1}(\lambda+v)^{\oplus m_{\xi}(v)} \hookrightarrow Q_{1}(\lambda) \otimes_{\mathbb{F}} L(\xi)
$$

which is injective since it is injective on socles. The morphism is hence an isomorphism by (4.6) (note that $\widehat{L}_{1}(\xi)_{v}=L(\xi)_{v}$ for all $v \in X_{1}(\underline{T})$ since $\xi \in X_{1}(\underline{T})$ ) and item (2) follows.

We prove the claim. It will be enough to prove that for any irreducible, $p$ bounded $\underline{G}$-module $L(\kappa)$, one has

$$
\operatorname{Ext}_{\underline{G}}^{1}\left(L(\kappa), Q_{1}(\lambda) \otimes_{\mathbb{F}} L(\xi)\right)=0 .
$$

As $L(\kappa)$ is $p$-bounded, we can write $\kappa=\kappa^{(0)}+p \omega_{\kappa}$ where $\kappa^{(0)} \in X_{1}(\underline{T})$ and $\omega_{\kappa} \in X_{+}^{*}\left(\underline{T)}\right.$ satisfies $\left\langle\omega_{\kappa}, \alpha^{\vee}\right\rangle<2(h-1)$ for all coroots $\alpha^{\vee} \in \underline{R}^{\vee}$. Recall that $\left.Q_{1}(\lambda)\right|_{\underline{G}_{1}} \cong \widehat{Q}_{1}(\lambda)$. As $\widehat{Q}_{1}(\lambda)$ is injective as a $\underline{G}_{1}$-module, the LyndonHochschild-Serre spectral sequence [Jan03, I.6.6(3), I.6.5(2)], together with (4.6), provides us with an isomorphism

$$
\begin{aligned}
\operatorname{Ext}_{\underline{G}}^{1}\left(L(\kappa), Q_{1}(\lambda) \otimes_{\mathbb{F}} L(\xi)\right) & \cong \operatorname{Ext}_{\underline{G} / \underline{G}_{1}}^{1}\left(L\left(p \omega_{\kappa}\right), \operatorname{Hom}_{\underline{G}_{1}}\left(L_{1}\left(\kappa^{(0)}\right), \widehat{Q}_{1}(\lambda) \otimes_{\mathbb{F}} L_{1}(\xi)\right)\right) \\
\cong & \operatorname{Ext}_{\underline{G} / \underline{G}_{1}}^{1}\left(L\left(p \omega_{\kappa}\right), \operatorname{Hom}_{\underline{G}_{1}}\left(L_{1}\left(\kappa^{(0)}\right), \bigoplus_{v \in \widehat{L}_{1}(\xi)} \widehat{Q}_{1}(\lambda+v)\right)^{\oplus m_{\xi}(v)}\right) \\
& \cong \begin{cases}\operatorname{Ext}_{\underline{G}}^{1}\left(L\left(\omega_{\kappa}\right), L\left(\frac{\lambda+v-\kappa^{(0)}}{p}\right)\right)^{\oplus m_{\xi}(v)} & \text { if } \lambda+v-\kappa^{(0)} \in p X^{0}(\underline{T}) \\
0 & \text { else. }\end{cases}
\end{aligned}
$$

As $\left\langle\omega_{\kappa}, \alpha^{\vee}\right\rangle<2(h-1) \leqslant p-2$ for all coroots $\alpha^{\vee} \in \underline{R}^{\vee}$, it follows that $\omega_{\kappa}$ lies in the lower $p$-restricted alcove; in particular, there are no algebraic extensions between $L\left(\omega_{\kappa}\right)$ and $L(\omega)$ for any $\omega \in X^{0}(\underline{T})$. This establishes (4.8). 
4.2.2. The case of $\mathrm{GL}_{3}$. We now describe the modules $\left.Q_{1}(\mu)\right|_{\mathrm{G}}$ in more detail in the case $\underline{G}_{0}$ is $\prod_{\tilde{v} \in \mathcal{S}} \operatorname{Res}_{k_{\tilde{v}} / \mathbb{F}_{p}} \mathrm{GL}_{3}$ as in Section 1.4 and $\mu \in X_{1}(\underline{T})$. We recall the alcove labeling for $\mathrm{SL}_{3}$ in [Hum06, Section 13.9] and write

$$
\mathscr{A} \stackrel{\text { def }}{=}\{A, B, C, D, E, F, G, H, I, J\}
$$

If $X \in \mathscr{A}$, let $\widetilde{w}_{X} \in W_{a}$ be the unique element such that $\widetilde{w}_{X} \cdot C_{0}=X$. For $i \in \mathcal{J}$, we define $\widetilde{w}_{X, i} \in \underline{W}_{a}$ in the evident way. For $\underline{X} \in \mathscr{A}^{\mathcal{J}}$, we also define $\widetilde{w}_{\underline{X}} \in \underline{W}_{a}$ in the evident way. In what follows, we let $f \stackrel{\text { def }}{=} \# \mathcal{J}$.

Assume from now on that $p \geqslant 5$ and $\mu$ is 2-deep. Let $\mu^{\mathrm{op}}$ be $\widetilde{w}_{\underline{B}} \cdot \mu$ and write $\mu^{\text {op }}$ as the sum $\sum_{i} \mu_{i}^{\text {op }}$. Let $Q_{1}\left(\mu_{i}\right)$ be the $\mathrm{GL}_{3 / \mathbb{F}}$-module defined in Theorem 4.2.1. It is rigid with Loewy length $\ell \ell\left(Q_{1}(\mu)\right)=6+1$ and is endowed with a Weyl filtration (see [Jan03, II.11.13, II.11.5(5), II.4.19] and also [Hum06, Section 13.9]) with submodule $V_{\mu_{i}} \stackrel{\text { def }}{=} V\left(\mu_{i}^{\text {op }}+p \eta_{i}^{\prime}\right)$ and the $\mathrm{GL}_{3 / \mathbb{F}}$-module obtained by extension of scalars from the Weyl module for $\mathrm{GL}_{3 / \mathbb{F}}$ with the highest weight $\mu_{i}^{\mathrm{op}}+p \eta_{i}^{\prime}$. Moreover, the socle filtration of $Q_{1}\left(\mu_{i}\right)$ (see [Hum06, Section 13.9] for a concise reference, [AM01, Proposition 8.4] and its proof and [Jan03, Section II.D.4]) and $V_{\mu_{i}}$ (cf. [BDM15, Section 4], and Table 6) is known. (The condition that $\mu$ is 2-deep is to guarantee that all $V_{\mu_{i}}$ has maximal length; their Loewy length in this case is $3+1$.) In particular, one sees that $V_{\mu_{i}}$ is a multiplicity-free submodule of $Q_{1}\left(\mu_{i}\right)$. Then the $G$-modules $Q_{1}(\mu)$ and $V_{\mu}$ are defined to be the tensor products $\bigotimes_{i} Q_{1}\left(\mu_{i}\right)$ and $\bar{\bigotimes}_{i} V_{\mu_{i}}$, respectively.

The module $Q_{1}(\mu)$ is rigid with Loewy length $\ell \ell\left(Q_{1}(\mu)\right)=6 f+1$. The socle filtration on $Q_{1}(\mu)$ is the tensor product of the socle filtrations Fil on $Q_{1}\left(\mu_{i}\right)$ for $i \in \mathcal{J}$, and the graph $\Gamma\left(V_{\mu}\right)$ is the product $\prod_{i} \Gamma\left(V_{\mu_{i}}\right)$. In particular, $V_{\mu}$ is rigid. Let Fil be the unique increasing Loewy series for $Q_{1}(\mu)$; its restriction to $V_{\mu}$ is the unique Loewy series for $V_{\mu}$.

Recall that an irreducible $\mathbb{F}[\mathrm{G}]$-module $F$ is said to be $n$-deep if we can write $\left.F \cong L(\mu)\right|_{\underline{G}\left(\mathbb{F}_{p}\right)}$ for some $\mu \in X_{1}(\underline{T})$ which is $n$-deep. A $\mathbb{F}[\mathrm{G}]$-module is defined to be $n$-deep if all its Jordan-Hölder constituents are $n$-deep.

LeMmA 4.2.5. Let $\mu \in X^{*}(\underline{T}), n \in \mathbb{N}$, and $\widetilde{w} \in \widetilde{W}$. If $\mu$ is $n$-deep in alcove $a$, then $\widetilde{w} \cdot \mu$ is $n$-deep in alcove $\widetilde{w} \cdot a$. In particular, if $\mu \in X_{1}(\underline{T})$ is $n$-deep, then $Q_{1}(\mu)$ and $V_{\mu}$ are $n$-deep. If $\mu \in X_{1}(\underline{T})$ is $n+2$-deep, then $\left.Q_{1}(\mu)\right|_{\mathrm{G}}$ and $\left.V_{\mu}\right|_{\mathrm{G}}$ are all $n$-deep.

Proof. The first two claims are easy. To prove the final claim, first note that the Jordan-Hölder factors of $\left.Q_{1}(\mu)\right|_{\mathrm{G}}$ and $\left.V_{\mu}\right|_{\mathrm{G}}$ are the same, and so it suffices to prove the claim for $\left.L\right|_{\mathrm{G}}$ where $L \in \mathrm{JH}\left(V_{\mu}\right)$. Suppose that $L$ is isomorphic to 
$L(\lambda)$ and $\lambda=\lambda^{0}+p \omega_{\lambda}$. Then

$$
\left.L\right|_{\mathrm{G}} \cong L\left(\lambda^{0}+\pi \omega_{\lambda}\right) \cong \bigoplus_{\varepsilon \in L\left(\pi \omega_{\lambda}\right)} L(\lambda+\varepsilon)^{m_{\pi \omega_{\lambda}}(\varepsilon)}
$$

by Lemma 4.2.4(1). The result now follows from the fact that $\left|\left\langle\varepsilon, \alpha^{\vee}\right\rangle\right| \leqslant 2$ for any $\varepsilon \in L\left(\pi \omega_{\lambda}\right)$ and any positive root $\alpha \in R^{+}$of a simple factor of $\underline{G}$.

Assume that $\mu$ is 6-deep. By Corollary 4.2.3, the socle filtration on $\operatorname{Inj}(F(\mu))$ is given by $\left.\left(\operatorname{Fil}_{n} Q_{1}(\mu)\right)\right|_{\mathrm{G}}$. Since $\operatorname{Inj}(F(\mu))$ is rigid (it is isomorphic to $\operatorname{Proj}(F(\mu)))$, this is the unique increasing Loewy series. One can use Lemma 4.2.4 to compute $\operatorname{gr}_{n} \operatorname{Inj}(F(\mu))$. We do this in the case $n=1$ to compute G-extensions, justifying the name 'extension graph' introduced in Section 2.

LEMMA 4.2.6. Assume that $\mu_{i}$ is 6-deep for all $i$ and let $\sigma \stackrel{\text { def }}{=} F(\mu)$. Then $\mathrm{d}_{\mathrm{gph}}(\sigma$, $\kappa)=1$ if and only if $\operatorname{Ext}_{\mathrm{G}}^{1}(\kappa, \sigma) \neq 0$, in which case the dimension of the $\operatorname{Ext}^{1}$ group is 1 .

Proof. Since $\mu$ is 6-deep, $Q_{1}(\mu)$ is 6-deep by Lemma 4.2 .5 so that $\left.Q_{1}(\mu)\right|_{\mathrm{G}} \cong$ $\operatorname{Inj}(F(\mu))$ by Corollary 4.2.3. It suffices to show that $\left[\left.\operatorname{gr}_{1} Q_{1}(\mu)\right|_{\mathrm{G}}: \kappa\right] \leqslant 1$ and that $\mathrm{d}_{\mathrm{gph}}(\sigma, \kappa)=1$ if and only if $\left[\left.\mathrm{gr}_{1} Q_{1}(\mu)\right|_{\mathrm{G}}: \kappa\right]=1$. Note that $\operatorname{gr}_{1} Q_{1}(\mu)=$ $\bigoplus_{i}\left(\operatorname{gr}_{1} Q_{1}\left(\mu_{i}\right)\right) \otimes \bigotimes_{j \neq i} L\left(\mu_{j}\right)$. Let $\widetilde{w}_{\mu}$ be the element of $W_{a}$ so that $\lambda \stackrel{\text { def }}{=} \widetilde{w}_{\mu}^{-1} \cdot \mu$ is in $\underline{A}$. The length of $\operatorname{gr}_{1} Q_{1}(\mu)$ is $3 f$ with Jordan-Hölder factors of the form $L(\widetilde{w} \cdot \mu)$ for $3 f$ choices of $\widetilde{w}$. Writing $\widetilde{w}$ as $t_{\omega_{-}} \widetilde{w}_{+}$with $\widetilde{w}_{+} \in \widetilde{W}_{1}^{+}$, the $3 f$ choices of $\widetilde{w}$ correspond to $\omega_{-}=0, \varepsilon_{1, i}^{\prime}$, or $\varepsilon_{2, i}^{\prime}$ for some $i \in \mathcal{J}$, with $\widetilde{w}_{+}$the unique element in $\underline{W}_{1}^{+}$so that $t_{\omega_{-}} \widetilde{w}_{+}$is in $\underline{W}_{a}$ and $\widetilde{w}_{+} \widetilde{w}_{\mu} \cdot \underline{A}=\widetilde{w}_{B, i} \widetilde{w}_{\mu} \cdot \underline{A}$.

It suffices to show that

$$
\left.L(\widetilde{w} \cdot \mu)\right|_{\mathrm{G}}
$$

is multiplicity free and contains exactly the weights of the form $F\left(\mathfrak{T r}_{\lambda+\eta}(\omega\right.$, $\left.\left.\pi\left(\widetilde{w}_{B, i} \widetilde{w}_{\mu} \cdot \underline{A}\right)\right)\right)$ with $\omega$ a permutation of $\pi \omega_{-}$. (Here, $\omega$ as the first argument of $\mathfrak{T r}_{\lambda+\eta}$ is understood to be the image of $\omega$ in $\Lambda_{W}$.)

We have isomorphisms

$$
\begin{aligned}
\left.L(\widetilde{w} \cdot \mu)\right|_{\mathrm{G}} & \left.\cong L\left(\widetilde{w}_{+} \cdot \mu\right) \otimes L\left(\pi \omega_{-, i}\right)\right|_{\mathrm{G}} \\
& \cong \bigoplus_{\omega \in L\left(\pi \omega_{-, i}\right)} F\left(\widetilde{w}_{+} \cdot \mu+\omega\right)^{\oplus m_{\pi \omega_{-}, i}(\omega)},
\end{aligned}
$$

where the second isomorphism follows from Lemma 4.2.4. On the other hand, the pair $\left(\omega, \pi\left(\widetilde{w}_{+, i} \widetilde{w}_{\mu} \cdot \underline{A}\right)\right)$ is $\beta\left(\omega, \widetilde{w}_{+, i} \widetilde{w}_{\mu}\right)$ with $\beta$ as in Lemma 2.1.1 since we have that $t_{\omega} \pi\left(\widetilde{w}_{+, i} \widetilde{w}_{\mu}\right) \in \underline{W}_{a}$. Then by definition, we have

$$
F\left(\mathfrak{T r}_{\lambda+\eta}\left(\omega, \pi\left(\widetilde{w}_{+} \widetilde{w}_{\mu} \cdot \underline{A}\right)\right)\right) \cong F\left(\widetilde{w}_{+} \widetilde{w}_{\mu} \cdot(\lambda+\omega)\right) \cong F\left(\widetilde{w}_{+} \cdot \mu+w_{+} w_{\mu} \omega\right),
$$


where $w_{+} w_{\mu}$ is the image of $\widetilde{w}_{+} \widetilde{w}_{\mu}$ in $\underline{W}$. We conclude by combining (4.9) and (4.10).

4.2.3. Study of the Weyl module $\left.V_{\mu}\right|_{\mathrm{G}}$. We now assume that $\mu \in X_{1}(\underline{T})$ is such that $\mu \in \underline{B}$ and push the analysis in Lemma 4.2.6 further to describe $\left.V_{\mu}\right|_{\mathrm{G}}$ in this case. Recall that $\underline{G}_{0} \stackrel{\text { def }}{=} \operatorname{Res}_{k_{\tilde{v}} / \mathbb{F}_{p}} \mathrm{GL}_{3}$ and that $\underline{G} \stackrel{\text { def }}{=} \underline{G}_{0} \times_{\mathbb{F}_{p}} \mathbb{F}$. Recall that $\underline{T} \subset \underline{G}$ is the diagonal torus, and $\underline{\Lambda}_{W}$ is the weight lattice of $\underline{G}^{\text {der }}$. Let $\underline{\Lambda}_{\eta^{\prime}} \subseteq \underline{\Lambda}_{W}$ be the convex hull of the $\underline{W}$-orbit of $\eta^{\prime}$. Explicitly, we have

$$
\begin{aligned}
\underline{\Lambda}_{\eta^{\prime}}= & \left\{\left(v_{i}\right)_{i} \in \underline{\Lambda}_{W}, v_{i} \in\left\{0, \pm \varepsilon_{1, i}, \pm \varepsilon_{2, i}, \pm \eta_{i}^{\prime}, \pm\left(\varepsilon_{1, i}-\varepsilon_{2, i}\right),\right.\right. \\
& \left.\left. \pm\left(2 \varepsilon_{1, i}-\varepsilon_{2, i}\right), \pm\left(\varepsilon_{1, i}-2 \varepsilon_{2, i}\right)\right\}\right\} .
\end{aligned}
$$

We define the subgraph $\underline{\Lambda}_{\leq\left(\eta^{\prime}, 0\right)} \subseteq \underline{\Lambda}_{\eta^{\prime}} \times \mathcal{A}$ as follows:

$$
\underline{\Lambda}_{\leq \eta^{\prime}, 0} \stackrel{\text { def }}{=}\left\{(\omega, a) \in \underline{\Lambda}_{\eta^{\prime}} \times \mathcal{A}: a_{i}=0 \text { if } \omega_{i}=w \eta_{i}^{\prime} \text { for some } w \in W\right\} .
$$

The main result concerning $V_{\mu}$ is the following.

THEOREM 4.2.7. Let $\mu \in X_{1}(\underline{T})$ be a p-restricted weight such that $\mu$ is 2-deep in alcove $\underline{B}$.

(1) The translation map $\mathfrak{T r}_{\mu^{\mathrm{op}+\eta}}: \underline{\Lambda}_{W}^{\left(\mu^{\mathrm{op}}+\eta\right)} \times \mathcal{A} \rightarrow X_{1}(\underline{T}) /\left\langle(p-\pi) X^{0}(\underline{T})\right\rangle$ induces a bijection:

$$
\begin{aligned}
\mathfrak{T}_{V_{\mu}}: \underline{\Lambda}_{\leq\left(\eta^{\prime}, 0\right)} & \rightarrow \mathrm{JH}\left(\left.V_{\mu}\right|_{\mathrm{G}}\right) \\
(\omega, a) & \mapsto \sigma_{(\omega, a)} .
\end{aligned}
$$

(2) We have

$$
\left[\left.\operatorname{gr}_{d}\left(V_{\mu}\right)\right|_{\mathrm{G}}: \sigma_{(\omega, a)}\right]= \begin{cases}1 & \text { if } d=\mathrm{d}_{\mathrm{gph}}\left(\sigma_{(0,1)}, \sigma_{(\omega, a)}\right) \\ 0 & \text { if } d<\mathrm{d}_{\mathrm{gph}}\left(\sigma_{(0,1)}, \sigma_{(\omega, a)}\right)\end{cases}
$$

for all $(\omega, a) \in \underline{\Lambda}_{\leq\left(\eta^{\prime}, 0\right)}$.

(3) Assume that $\mu$ is 6-deep. Then there exists a G-submodule $\left.U \subset V_{\mu}\right|_{\mathrm{G}}$ such that:

(a) $U$ is multiplicity free;

(b) $\mathrm{JH}(U)=\mathrm{JH}\left(\left.V_{\mu}\right|_{\mathrm{G}}\right)$;

(c) if we denote the restriction Fil $\left.\right|_{U}$ by Fil, for any $\sigma \in \mathrm{JH}(U)$, we have $\left[\mathrm{gr}_{d}(U): \sigma\right]=1$ if and only if $d=\mathrm{d}_{\mathrm{gph}}\left(\sigma_{(0,1)}, \sigma\right)$; and 
(d) if $(\omega, a),\left(\omega^{\prime}, a^{\prime}\right) \in \underline{\Lambda}_{\leq\left(\eta^{\prime}, 0\right)}$, then $\sigma_{(\omega, a)}$ points to $\sigma_{\left(\omega^{\prime}, a^{\prime}\right)}$ (with respect to $U$ ) if and only if $\mathrm{d}_{\mathrm{gph}}\left(\sigma_{(0,1)}, \sigma_{(\omega, a)}\right) \geqslant \mathrm{d}_{\mathrm{gph}}\left(\sigma_{(0,1)}, \sigma_{\left(\omega^{\prime}, a^{\prime}\right)}\right)$, and $\mathrm{d}_{\mathrm{gph}}\left(\sigma_{(\omega, a)}, \sigma_{\left(\omega^{\prime}, a^{\prime}\right)}\right)=1$. In particular, $\Gamma(U)^{\mathrm{op}}$ (that is, the graph obtained from $\Gamma(U)$ by reversing the direction of the edges) is predicted by the extension graph with respect to $\sigma_{(0,1)}$.

Proof of 4.2.7(1) and (2). We first show that the image of $\mathfrak{T r}_{V_{\mu}}$ contains $\operatorname{JH}\left(\left.V_{\mu}\right|_{\mathrm{G}}\right)$. Let $\underline{\widetilde{w}} \in \underline{W}_{a}$ such that $\left[V_{\mu}: L(\widetilde{w} \cdot \mu)\right] \neq 0$. It suffices to show that $\mathfrak{T r}_{V_{\mu}}$ contains

$$
\mathrm{JH}\left(\left.L(\widetilde{w} \cdot \mu)\right|_{\mathrm{G}}\right)
$$

The proof is similar to that of Lemma 4.2.6. There is a decomposition $\widetilde{w}=t_{\omega_{-}} \widetilde{w}_{+}$ where $\widetilde{w}_{+} \in \widetilde{W}_{1}^{+}$. Again, $\left.L(\widetilde{w} \cdot \mu)\right|_{\mathrm{G}}$ is isomorphic to $\left.L\left(\widetilde{w}_{+} \cdot \mu\right) \otimes L\left(\pi \omega_{-}\right)\right|_{\mathrm{G}}$, which is isomorphic to

$$
\bigoplus_{\varepsilon \in L\left(\pi \omega_{-}\right)} F\left(\widetilde{w}_{+} \cdot \mu+\varepsilon\right)^{\oplus m_{\pi \omega_{-}}(\varepsilon)}
$$

by Lemma 4.2.4. As in the proof of Lemma 4.2.6, the summand $F\left(\widetilde{w}_{+} \cdot \mu+\varepsilon\right)$ is $\sigma_{\left(w_{0} \varepsilon, \pi\left(\widetilde{w}_{+} \cdot \widetilde{w}_{h} \cdot \underline{A}\right)\right)}$. Then (4.11) is contained in the image of $\mathfrak{T r}_{V_{\mu}}$ by an analysis of the weights of $L\left(\pi \omega_{-}\right)$. Indeed, $\widetilde{w} \cdot \mu$ is in one of the alcoves in the set $\{A$, $B, C, D, E, F, G\}^{\mathcal{J}}$ so that $\omega_{-, i}$ can be taken to be one of $0, \varepsilon_{1, i}, \varepsilon_{2, i}$, and $\eta_{i}^{\prime}$ for all $i$, and if $\omega_{-, i}$ is $\eta_{i}^{\prime}$, then $\widetilde{w}_{+, i}=\widetilde{w}_{B, i}$.

Item (1) follows from (2) and the above paragraph. We now prove item (2). With $\widetilde{w}$ as above, we define $n_{i}\left(\widetilde{w}_{i}\right) \in \mathbb{N}$ by

$$
\left[\operatorname{gr}_{n_{i}\left(\widetilde{w}_{i}\right)}\left(V_{\mu_{i}}\right): L\left(\widetilde{w}_{i} \cdot \mu_{i}\right)\right] \neq 0 .
$$

Let $0 \leqslant d \leqslant 3 f$. Since $V_{\mu}$ is multiplicity free, it suffices to show that

$$
\left.\bigoplus_{\sum_{i} n_{i}\left(\widetilde{w}_{i}\right)=d} L(\widetilde{w} \cdot \mu)\right|_{\mathrm{G}}
$$

contains weights of the form $\sigma_{(\omega, a)}$ with multiplicity one if $(\omega, a) \in \underline{\Lambda}_{\leq\left(\eta^{\prime}, 0\right)}$ and the distance between $\left(\omega_{i}, a_{i}\right)$ and $\left(0_{i}, 1_{i}\right)$ is $n_{\pi^{-1} i}\left(\widetilde{w}_{\pi^{-1} i}\right)$ for all $i$. If $v \in L\left(\pi \omega_{-}\right)$ is a permutation of $\pi \omega_{-}$, then it appears in $L\left(\pi \omega_{-}\right)$with multiplicity one. Thus, $\sigma_{\left(\varepsilon, \pi\left(\widetilde{w}_{+} \widetilde{w}_{h}\right) \cdot \underline{A}\right)}$ appears in (4.12) with multiplicity one. A casewise analysis, using the fifth column of Table 5 and the description of the socle layers of $V_{\mu_{i}}$ in [BDM15], shows that the distance from $\left(v_{i}, \pi\left(\widetilde{w}_{+, \pi^{-1} i} \cdot \widetilde{w}_{B, \pi^{-1} i}\right) \cdot A\right)$ to $\left(0_{i}, 1_{i}\right)$ is $n_{\pi^{-1} i}\left(\widetilde{w}_{\pi^{-1} i}\right)$. For example, if $n_{\pi^{-1} i}\left(\widetilde{w}_{\pi^{-1} i}\right)=2$, then $\omega_{-, \pi^{-1} i}$ is $\varepsilon_{1, \pi^{-1} i}^{\prime}$ or $\varepsilon_{2, \pi^{-1} i}^{\prime}$ and $\widetilde{w}_{+, \pi^{-1} i}$ is trivial. Then $v_{i}$ is a permutation of $\varepsilon_{1, i}$ or $\varepsilon_{2, i}$.

We now move to the proof of Theorem 4.2.7(3). We start with the following preliminary lemma. 
Table 5. Comparison between alcoves $C_{i}$, affine Weyl group elements $\widetilde{w}_{i}$ and the graph.

\begin{tabular}{|c|c|c|c|c|}
\hline$\widetilde{w}_{i}$ & $\widetilde{w}_{+, i}$ & $\omega_{-, i}$ & $\widetilde{w}_{i} \cdot A$ & $(\omega, a)_{\pi i}$ \\
\hline id & id & $t_{0}$ & $A$ & $(0,0)$ \\
\hline$(13) t_{-\left(\varepsilon_{1}^{\prime}+\varepsilon_{2}^{\prime}\right)}$ & $(13) t_{-\left(\varepsilon_{1}^{\prime}+\varepsilon_{2}^{\prime}\right)}$ & $t_{0}$ & $B$ & $(0,1)$ \\
\hline$(123) t_{-\alpha_{2}}$ & $(123) t_{-\varepsilon_{2}^{\prime}}$ & $t_{\varepsilon_{2}^{\prime}}$ & $C$ & $\left(s\left(\varepsilon_{2}^{\prime}\right), 0\right) ; s \in S_{3}$ \\
\hline$(12) t_{\alpha_{2}}$ & $\left.(12) t_{-\left(\varepsilon_{1}^{\prime}-\varepsilon_{2}^{\prime}-1\right.}\right)$ & $t_{\varepsilon_{2}^{\prime}}$ & $E$ & $\left(s\left(\varepsilon_{2}^{\prime}\right), 1\right) ; s \in S_{3}$ \\
\hline$(132) t_{-\alpha_{1}}$ & $(132) t_{-\varepsilon_{1}^{\prime}}$ & $t_{\varepsilon_{1}^{\prime}}$ & $D$ & $\left(s\left(\varepsilon_{1}^{\prime}\right), 0\right) ; s \in S_{3}$ \\
\hline$(23) t_{\alpha_{1}}$ & $(23) t_{-\left(\varepsilon_{2}^{\prime}-\varepsilon_{1}^{\prime}+\underline{1}\right)}$ & $t_{\varepsilon_{1}^{\prime}}$ & $F$ & $\left(s\left(\varepsilon_{1}^{\prime}\right), 1\right) ; s \in S_{3}$ \\
\hline$(13) t_{-2\left(\varepsilon_{1}^{\prime}+\varepsilon_{2}^{\prime}\right)}$ & $(13) t_{-\left(\varepsilon_{1}^{\prime}+\varepsilon_{2}^{\prime}\right)}$ & $t_{\left(\varepsilon_{1}^{\prime}+\varepsilon_{2}^{\prime}\right)}$ & $J$ & $\left(s\left(\varepsilon_{1}^{\prime}+\varepsilon_{2}^{\prime}\right), 1\right) ; s \in S_{3}$ and $(0,1)$ \\
\hline$\widetilde{w}_{i}$ & $\widetilde{w}_{i,+}$ & $\widetilde{w}_{i,-}$ & $\widetilde{w}_{i} \cdot B$ & $(\omega, a)_{\pi i}$ \\
\hline id & id & $t_{0}$ & $B$ & $(0,1)$ \\
\hline$(13) t_{-\left(\varepsilon_{1}^{\prime}+\varepsilon_{2}^{\prime}\right)}$ & $(13) t_{-\left(\varepsilon_{1}^{\prime}+\varepsilon_{2}^{\prime}\right)}$ & $t_{0}$ & $A$ & $(0,0)$ \\
\hline$(132) t_{-\left(2 \alpha_{1}+\alpha_{2}\right)}$ & $(132) t_{-\left(2 \varepsilon_{1}^{\prime}-1\right)}$ & $t_{\varepsilon_{2}^{\prime}}$ & $E$ & $\left(s\left(\varepsilon_{2}^{\prime}\right), 1\right) ; s \in S_{3}$ \\
\hline$(23) t_{-\alpha_{2}}$ & $(23) t_{-\varepsilon_{2}^{\prime}}$ & $t_{\varepsilon_{2}^{\prime}}$ & $C$ & $\left(s\left(\varepsilon_{2}^{\prime}\right), 0\right) ; s \in S_{3}$ \\
\hline$(123) t_{-\left(\alpha_{1}+2 \alpha_{2}\right)}$ & $(123) t_{-\left(2 \varepsilon_{2}^{\prime}+1\right)}$ & $t_{\varepsilon_{1}^{\prime}}$ & $F$ & $\left(s\left(\varepsilon_{1}^{\prime}\right), 1\right) ; s \in S_{3}$ \\
\hline$(12) t_{-\alpha_{1}}$ & $(12) t_{-\varepsilon_{1}^{\prime}}$ & $t_{\varepsilon_{1}^{\prime}}$ & $D$ & $\left(s\left(\varepsilon_{1}^{\prime}\right), 0\right) ; s \in S_{3}$ \\
\hline$(13) t_{-2\left(\varepsilon_{1}^{\prime}+\varepsilon_{2}^{\prime}\right)}$ & $(13) t_{-\left(\varepsilon_{1}^{\prime}+\varepsilon_{2}^{\prime}\right)}$ & $t_{\left(\varepsilon_{1}^{\prime}+\varepsilon_{2}^{\prime}\right)}$ & $G$ & $\left(s\left(\varepsilon_{1}^{\prime}+\varepsilon_{2}^{\prime}\right), 0\right) ; s \in S_{3}$ and $(0,0)$ \\
\hline
\end{tabular}

For each element $\widetilde{w}^{*}$ in the first column, we consider the decomposition $\widetilde{w}_{i}=\omega_{i,-}$. $\widetilde{w}_{i,+}$ with $\left(\widetilde{w}_{i,+}\right)_{i} \in \widetilde{W}^{+}$. In the fourth column, we write the alcove containing $\widetilde{w}_{i}$. $A$. In the fifth column, we write the $\pi i$ th coordinate of the points in the graph (with origin $\left.\mu+\eta^{\prime}\right)$ corresponding to an irreducible constituent of $\left.\left(\bigotimes_{i} L\left(\widetilde{w}_{i} \cdot \mu_{i}\right)\right)\right|_{\mathrm{G}}$. Similar comments apply to the second half of the table. Note that in this case, we consider the decomposition $\widetilde{w}_{i}=\omega_{-, i} \cdot \widetilde{w}_{+, i}$ with $\widetilde{w}_{+, i} \in \widetilde{W}_{1}^{+} \widetilde{w}_{B}$ and the graph has origin in $\mu^{\mathrm{op}}+\eta$. Finally, we have set $\alpha_{1}$ and $\alpha_{2} \in X^{*}(T)$ to be $(1,-1,0)$ and $(0,1,-1)$, respectively.

LEMMA 4.2.8. Let $\mu \in X_{1}(\underline{T)}$ be a p-restricted weight which is 2-deep in alcove B. There exists a $\mathrm{G}$-submodule $\left.U \subset V_{\mu}\right|_{\mathrm{G}}$ such that $\mathrm{JH}(U)=\mathrm{JH}\left(\left.V_{\mu}\right|_{\mathrm{G}}\right)$ and

$$
\left[\left.\operatorname{gr}_{d}(U)\right|_{\mathrm{G}}: \sigma_{(\omega, a)}\right]= \begin{cases}1 & \text { if } d=\mathrm{d}_{\mathrm{gph}}\left(\sigma_{(0,1)}, \sigma_{(\omega, a)}\right) \\ 0 & \text { if } d \neq \mathrm{d}_{\mathrm{gph}}\left(\sigma_{(0,1)}, \sigma_{(\omega, a)}\right)\end{cases}
$$

where gr is with respect to $\left.\mathrm{Fil} \stackrel{\text { def }}{=} \mathrm{Fil}\right|_{U}$. In particular, $U$ is multiplicity free.

Proof. The notation in this proof is complicated by necessity. To illustrate the simple underlying idea, we first present the proof in the case that $f=\# \mathcal{J}=1$. We have the following:

$$
\begin{aligned}
& \operatorname{gr}_{3}\left(V_{\mu}\right) \cong L\left(\widetilde{w}_{G} \widetilde{w}_{B} \cdot \mu\right) \\
& \operatorname{gr}_{2}\left(V_{\mu}\right) \cong L\left(\widetilde{w}_{E} \widetilde{w}_{B} \cdot \mu\right) \oplus L\left(\widetilde{w}_{F} \widetilde{w}_{B} \cdot \mu\right)
\end{aligned}
$$


Table 6. Graph of $V\left(\mu^{\text {op }}+p \eta^{\prime}\right)$.

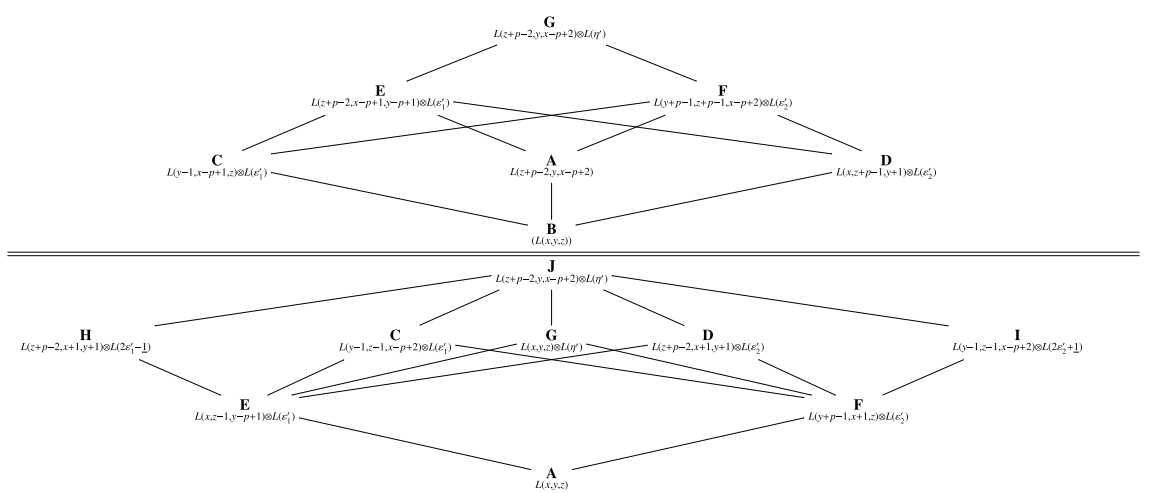

The graphs of $V\left(\mu^{\mathrm{op}}+p \eta^{\prime}\right)$ when $f=1$ (and $\mu$ is 2-deep). In the first (respectively second) diagram, $\mu \in X_{1}(\underline{T)}$ is upper alcove (respectively lower alcove). For each alcove, we write below the unique weight $L$ such that $L(x, y, z) \uparrow L$. We remark that $\operatorname{gr}_{1}\left(V\left(\mu^{\text {op }}+p \eta^{\prime}\right)=\operatorname{gr}_{1}\left(Q_{1}(\mu)\right)\right.$ if $\mu=(x, y, z)$ is upper alcove, while $\operatorname{gr}_{1}\left(V\left(\mu^{\text {op }}+\right.\right.$ $\left.p \eta^{\prime}\right) \oplus L\left(\mu^{\mathrm{op}}\right)=\operatorname{gr}_{1}\left(Q_{1}(\mu)\right)$ if $\mu=(x, y, z)$ is lower alcove.

$$
\begin{aligned}
& \operatorname{gr}_{1}\left(V_{\mu}\right) \cong L\left(\widetilde{w}_{C} \widetilde{w}_{B} \cdot \mu\right) \oplus L\left(\widetilde{w}_{B} \cdot \mu\right) \oplus L\left(\widetilde{w}_{D} \widetilde{w}_{B} \cdot \mu\right) \\
& \operatorname{gr}_{0}\left(V_{\mu}\right) \cong L(\mu)
\end{aligned}
$$

For the alcove $a$, there is a decomposition $\widetilde{w}_{a}$ as the product $t_{\omega_{a,-}} \widetilde{w}_{a,+}$ where $\widetilde{w}_{a,+} \in \widetilde{W}_{1}^{+}$and $\omega_{a,-} \in X^{*}(T)$. Let $\lambda$ be $\widetilde{w}_{B} \cdot \mu$. Then

$$
\begin{aligned}
\left.L\left(\widetilde{w}_{a} \widetilde{w}_{B} \cdot \mu\right)\right|_{\mathrm{G}} & \left.\cong L\left(\widetilde{w}_{a} \cdot \lambda\right) \cong L\left(\widetilde{w}_{a,+} \cdot \lambda+p \omega_{a,-}\right)\right|_{\mathrm{G}} \\
& \left.\cong\left(L\left(\widetilde{w}_{a,+} \cdot \lambda\right) \otimes L\left(p \omega_{a,-}\right)\right)\right|_{\mathrm{G}} \\
& \left.\cong\left(L\left(\widetilde{w}_{a,+} \cdot \lambda\right) \otimes L\left(\omega_{a,-}\right)\right)\right|_{\mathrm{G}} \\
& \left.\cong \bigoplus_{\omega \in L\left(\omega_{a,-}\right)} L\left(\widetilde{w}_{a,+} \cdot \lambda+\omega\right)\right|_{\mathrm{G}} ^{\oplus m_{\omega_{a,-}}(\omega)} \\
& \cong \bigoplus_{\omega \in L\left(\omega_{a,-}\right)} F\left(\mathfrak{T r}_{\lambda+\eta}(\omega, \bar{a})\right)^{\oplus m_{\omega_{a,-}}(\omega)}
\end{aligned}
$$

where $\bar{a}$ is the unique $p X^{*}(T)$-translate of $a$ lying in $\{A, B\}$. The third isomorphism above follows from the Steinberg tensor product theorem, and the fifth isomorphism follows from Lemma 4.2.4(1). We see then that $\left.\operatorname{gr}_{1}\left(V_{\mu}\right)\right|_{\mathrm{G}}$ is isomorphic to the multiplicity-free direct sum of $F\left(\mathfrak{T r}_{\lambda+\eta}(\omega, 0)\right)$ where $\omega$ is a permutation of $0, \varepsilon_{1}$, or $\varepsilon_{2}$. Similarly, $\left.\operatorname{gr}_{2}\left(V_{\mu}\right)\right|_{\mathrm{G}}$ is isomorphic to the multiplicityfree direct sum of $F\left(\mathfrak{T r}_{\lambda+\eta}(\omega, 1)\right)$ where $\omega$ is a permutation of $\varepsilon_{1}$ or $\varepsilon_{2}$. Finally, 
$\left.\operatorname{gr}_{3}\left(V_{\mu}\right)\right|_{\mathrm{G}}$ is isomorphic to $\bigoplus_{\omega \in L\left(\eta^{\prime}\right)} F\left(\mathfrak{T r}_{\lambda+\eta}(\omega, 0)\right)^{m_{\eta^{\prime}}(\omega)}$. Then, we can take $U$ to be the preimage of $\bigoplus_{\omega \in L\left(\eta^{\prime}\right)} F\left(\mathfrak{T}_{\mathfrak{r}+\eta}(\omega, 0)\right)$ in $\left.V_{\mu}\right|_{\mathrm{G}}$. It is then easy to check that $U$ satisfies the required properties.

We now proceed to the general case. Let $i \in \mathcal{J}$. We have an exact sequence of $\underline{G}$-modules

$$
0 \rightarrow \operatorname{rad}\left(V_{\mu_{i}}\right) \rightarrow V_{\mu_{i}} \stackrel{p_{i}}{\rightarrow} L\left(\mu_{i}^{\mathrm{op}}+p \eta_{i}^{\prime}\right) \rightarrow 0
$$

which gives the exact sequence

$$
0 \rightarrow \operatorname{rad}\left(V_{\mu_{i}}\right) \otimes \bigotimes_{j \neq i} V_{\mu_{j}} \rightarrow V_{\mu} \rightarrow L\left(\mu_{i}^{\mathrm{op}}+p \eta_{i}^{\prime}\right) \otimes \bigotimes_{j \neq i} V_{\mu_{j}} \rightarrow 0 .
$$

Then $\left.L\left(\mu_{i}^{\mathrm{op}}+p \eta_{i}^{\prime}\right) \otimes \bigotimes_{j \neq i} V_{\mu_{j}}\right|_{\mathrm{G}}$ is isomorphic to

$$
\left.L\left(\mu_{i}^{\mathrm{op}}\right) \otimes\left(V_{\mu_{\pi i}} \otimes L\left(\eta_{\pi i}^{\prime}\right)\right) \otimes \bigotimes_{j \neq i, \pi i} V_{\mu_{j}}\right|_{\mathrm{G}}
$$

Then we claim that

$$
V_{\mu_{\pi i}} \otimes L\left(\eta_{\pi i}^{\prime}\right) \cong \bigoplus_{\omega \in L\left(\eta_{\pi i}^{\prime}\right)} V_{\mu_{\pi i}+\omega}^{\oplus m_{\eta_{\pi i}^{\prime}}(\omega)}
$$

as $\underline{G}$-representations. We have an embedding

$$
V_{\mu_{\pi i}} \otimes L\left(\eta_{\pi i}^{\prime}\right) \hookrightarrow Q_{1}\left(\mu_{\pi i}\right) \otimes L\left(\eta_{\pi i}^{\prime}\right) \cong \bigoplus_{\omega \in L\left(\eta_{\pi i}^{\prime}\right)} Q_{1}\left(\mu_{\pi i}+\omega\right)^{\oplus m_{\eta_{\pi i}^{\prime}}(\omega)},
$$

where the last isomorphism follows from Lemma 4.2.4. Since

(1) $V_{\mu_{\pi i}} \otimes L\left(\eta_{\pi i}^{\prime}\right)$ contains all the Jordan-Hölder factors of the right-hand side of (4.14) with highest weights in alcove $G$ (using Lemma 4.2.4) and

(2) $\bigoplus_{\omega \in L\left(\eta_{\pi i}^{\prime}\right)} V_{\mu_{\pi i}+\omega}^{\oplus m_{\eta_{\pi i}^{\prime}}(\omega)}$ is the minimal submodule of the right-hand side of (4.14) containing all the Jordan-Hölder factors (counted with multiplicities) with highest weights in alcove $G$ (using the cosocle filtration of $V_{\mu_{\pi i}+\omega}$,

there is an injective map $\bigoplus_{\omega \in L\left(\eta_{\pi i}^{\prime}\right)} V_{\mu_{\pi i}+\omega}^{\oplus m_{\eta_{\pi i}^{\prime}}(\omega)} \hookrightarrow V_{\mu_{\pi i}} \otimes L\left(\eta_{\pi i}^{\prime}\right)$ which must be an isomorphism since the domain and codomain have the same length.

Let $U_{i}$ be the preimage of

$$
\left.\bigoplus_{\omega \in L\left(\eta_{i}^{\prime}\right), \omega \neq 0} L\left(\mu_{i}^{\mathrm{op}}\right) \otimes V_{\mu_{\pi i}+\omega} \otimes \bigotimes_{j \neq i, \pi i} V_{\mu_{j}}\right|_{\mathrm{G}}
$$


in $\left.V_{\mu}\right|_{\mathrm{G}}$. Let $U$ be the intersection $\cap_{i} U_{i}$. We claim that $U$ has the desired properties. If $d<\mathrm{d}_{\mathrm{gph}}\left(\sigma_{(0,1)}, \sigma_{(\omega, a)}\right)$, then (4.13) holds by Theorem 4.2.7(2). If $d=\mathrm{d}_{\mathrm{gph}}\left(\sigma_{(0,1)}, \sigma_{(\omega, a)}\right)$, then $\left[\operatorname{gr}_{d}\left(\left.V_{\mu}\right|_{\mathrm{G}}\right): \sigma_{(\omega, a)}\right]=1$ and $\left[\operatorname{gr}_{d}\left(U_{i}\right): \sigma_{(\omega, a)}\right]=1$ for all $i$ by the proof of Theorem 4.2.7(2). We conclude that $\left[\operatorname{gr}_{d}(U): \sigma_{(\omega, a)}\right]=1$.

We now suppose that $d>\mathrm{d}_{\mathrm{gph}}\left(\sigma_{(0, \underline{1})}, \sigma_{(\omega, a)}\right)$ and use the notation of the proof of Theorem 4.2.7(2). Suppose that $\sigma_{(\omega, a)}$ is a Jordan-Hölder factor of $\left.L(\widetilde{w} \cdot \mu)\right|_{\mathrm{G}} \cap \operatorname{gr}_{d}(U)$. Then there is some $i$ such that $n_{\pi^{-1} i}\left(\widetilde{w}_{\pi^{-1} i}\right)>d_{i}$ where $d_{i}$ is the distance from $\left(\omega_{i}, a_{i}\right)$ to $\left(0_{i}, 1_{i}\right)$. More precisely, $n_{\pi^{-1} i}\left(\widetilde{w}_{\pi^{-1} i}\right)$ is 3 and $\left(\omega_{i}, a_{i}\right)$ is $\left(0_{i}, 0_{i}\right)$. However, one can check as in the proof of Theorem 4.2.7(2) that $\left.L(\tilde{w} \cdot \mu)\right|_{\mathrm{G}} \cap \operatorname{gr}_{d}\left(U_{\pi^{-1} i}\right)$ does not contain any weight of the form $\sigma_{(\omega, a)}$ with $\left(\omega_{i}, a_{i}\right)=\left(0_{i}, 0_{i}\right)$.

Proposition 4.2.9. Let $\lambda, \theta$ be 6-deep in an alcove in $\{A, B, C, D, E$, $F, G\}^{\mathcal{J}}$. Then $\operatorname{Ext}_{G}^{1}(L(\theta), L(\lambda))$ is at most one-dimensional, and it is onedimensional if and only if $\lambda$ and $\theta$ are linked and lie in adjacent alcoves (that is, there exists $i_{0} \in \mathcal{J}$ such that $\lambda_{i}=\theta_{i}$ for all $i \neq i_{0}$ and $\lambda_{i_{0}}$ and $\theta_{i_{0}}$ lie in different alcoves sharing a face).

Proof. We immediately reduce to the case of $\mathrm{GL}_{3}$. Using (4.3), it suffices to consider the case of $\mathrm{SL}_{3}$, where the result follows from [And87, Section 4.1].

Let $\mu \in X_{1}(\underline{T})$ be a $p$-restricted weight 6-deep in alcove $\underline{B}$. Let $\widetilde{w}, \tilde{y} \in \underline{W}_{a}$ be elements such that the alcoves containing $\lambda \stackrel{\text { def }}{=} \widetilde{w} \cdot \mu, \theta \stackrel{\text { def }}{=} \widetilde{y} \cdot \mu$ are in $\{A, B$, $C, D, E, F, G\}^{\mathcal{J}}$ and $\operatorname{Ext}_{G}^{1}(L(\theta), L(\lambda)) \neq 0$. Note that $\lambda, \theta$ are both 6-deep in their alcove by Lemma 4.2.5.

Let $i_{0} \in \mathcal{J}$ be as in Proposition 4.2.9. Let $M$ be a nonsplit extension of $L(\theta)$ by $L(\lambda)$, which is unique up to isomorphism by Proposition 4.2.9.

Proposition 4.2.10. If $F_{0}$ and $F_{1}$ are in the socle and cosocle of $\left.M\right|_{\mathrm{G}}$, respectively, with $\left[\left.M\right|_{\mathrm{G}}: F_{0}\right]=1=\left[\left.M\right|_{\mathrm{G}}: F_{1}\right]$ and $\mathrm{d}_{\mathrm{gph}}\left(F_{0}, F_{1}\right)=1$, then there is a subquotient of $\left.M\right|_{\mathrm{G}}$ which is a nonsplit extension, unique up to isomorphism by Lemma 4.2.6, of $F_{1}$ by $F_{0}$.

Proposition 4.2.10 will be proven in several steps. Let $\lambda$ be $\lambda^{0}+p \omega_{\lambda}$ and $\theta=\theta^{0}+p \omega_{\theta}$ so that $\lambda^{0}$ and $\theta^{0}$ are in $X_{1}(\underline{T})$ and $\omega_{\lambda}$ and $\omega_{\theta}$ belong to $\left\{0, \varepsilon_{1, i}^{\prime}\right.$, $\left.\varepsilon_{2, i}^{\prime}, \eta_{i}^{\prime}\right\}^{\mathcal{J}}$. We begin with an algebraization lemma.

LEMMA 4.2.11. With $M$ and $i_{0}$ defined as above, assume, moreover, that $\lambda_{i}$ is p-restricted for all $i \neq i_{0}$. Then there is an injection $M \hookrightarrow Q_{1}\left(\lambda^{0}\right) \otimes L\left(p \omega_{\lambda}\right) \cong$ $\left(\otimes_{i \neq i_{0}} Q_{1}\left(\lambda_{i}\right)\right) \otimes Q_{1}\left(\lambda_{i_{0}}^{0}\right) \otimes L\left(p \omega_{\lambda}\right)$ whose restriction to $\mathrm{G}$ is an injective hull. 
Proof. The proof follows closely the argument of [Pil97, Lemma 3.1] (which is, in turn, based on [And87, Lemma 2.2]). There is an injection

$$
\operatorname{soc} M \cong L\left(\lambda^{0}\right) \otimes L\left(p \omega_{\lambda}\right) \hookrightarrow Q_{1}\left(\lambda^{0}\right) \otimes L\left(p \omega_{\lambda}\right) .
$$

This extends to an injection $M \hookrightarrow Q_{1}\left(\lambda^{0}\right) \otimes L\left(p \omega_{\lambda}\right)$ since

$$
\operatorname{Ext}_{\underline{G}}^{1}\left(L(\theta), Q_{1}\left(\lambda^{0}\right) \otimes L\left(p \omega_{\lambda}\right)\right)=0
$$

by the proof of (4.8). Both $Q_{1}\left(\lambda^{0}\right) \otimes L\left(p \omega_{\lambda}\right)$ and $M$ are 6-deep by Lemma 4.2.5. By Lemma 4.2.2, the restriction to $\mathrm{G}$ of the map $M \hookrightarrow Q_{1}\left(\lambda^{0}\right) \otimes L\left(p \omega_{\lambda}\right)$ is an isomorphism on socles and is, thus, essential. The restriction $\left.Q_{1}\left(\lambda^{0}\right) \otimes L\left(p \omega_{\lambda}\right)\right|_{\mathrm{G}}$ is an injective object by Lemma 4.2.4 and Theorem 4.2.1.

We begin with the following special case of Proposition 4.2.10, from which the general case will follow by the translation principle.

PROpOSITION 4.2.12. If $\lambda_{i}$ is $p$-restricted for all $i \neq i_{0}$, the conclusion of Proposition 4.2.10 holds.

Proof. We assume that $\mathrm{d}_{\mathrm{gph}}\left(F_{0}, F_{1}\right)=1$. Fix nonzero maps $\left.\operatorname{Proj}\left(F_{1}\right) \rightarrow M\right|_{\mathrm{G}}$ and $\left.M\right|_{\mathrm{G}} \rightarrow \operatorname{Inj}\left(F_{0}\right)$, unique up to scalar. If suffices to show that the composition

$$
\left.\operatorname{Proj}\left(F_{1}\right) \rightarrow M\right|_{\mathrm{G}} \rightarrow \operatorname{Inj}\left(F_{0}\right)
$$

is nonzero. The first map factors through $\operatorname{Proj}\left(F_{1}\right) / \operatorname{rad}^{2}\left(\operatorname{Proj}\left(F_{1}\right)\right)$ since the Loewy length of $\left.M\right|_{\mathrm{G}}$ is 2 . The second map factors as $\left.M\right|_{\mathrm{G}} \hookrightarrow \operatorname{Inj}\left(\left.M\right|_{\mathrm{G}}\right) \rightarrow$ $\operatorname{Inj}\left(F_{0}\right)$ where the second map is a projection to a direct summand. Applying gr $_{1}$ to the composition

$$
\operatorname{Proj}\left(F_{1}\right) /\left.\operatorname{rad}^{2}\left(\operatorname{Proj}\left(F_{1}\right)\right) \rightarrow M\right|_{\mathrm{G}} \hookrightarrow \operatorname{Inj}\left(\left.M\right|_{\mathrm{G}}\right) \rightarrow \operatorname{Inj}\left(F_{0}\right),
$$

we obtain

$$
F_{1} \hookrightarrow \operatorname{gr}_{1}\left(\left.M\right|_{\mathrm{G}}\right) \hookrightarrow \operatorname{gr}_{1} \operatorname{Inj}\left(\left.M\right|_{\mathrm{G}}\right) \rightarrow \operatorname{gr}_{1} \operatorname{Inj}\left(F_{0}\right),
$$

where the grading is with respect to the socle filtration. It suffices to show that the composition (4.16) is nonzero.

By Lemma 4.2.4, $F_{0}$ (respectively $F_{1}$ ) is isomorphic to $F\left(\lambda^{\prime}\right)$ where $\lambda^{\prime}=\lambda^{0}+$ $\varepsilon_{\lambda}$ (respectively $F\left(\theta^{0}+\varepsilon_{\theta}\right)$ ) for some $\varepsilon_{\lambda} \in L\left(\pi \omega_{\lambda}\right)$ (respectively $\varepsilon_{\theta} \in L\left(\pi \omega_{\theta}\right)$ ). Then using Lemma 4.2.11, (4.16) can be rewritten as

$$
\left.\left.\left.\left.L\left(\theta^{0}+\varepsilon_{\theta}\right)\right|_{\mathrm{G}} \hookrightarrow L(\theta)\right|_{\mathrm{G}} \hookrightarrow\left(\operatorname{gr}_{1}\left(Q_{1}\left(\lambda^{0}\right) \otimes L\left(p \omega_{\lambda}\right)\right)\right)\right|_{\mathrm{G}} \rightarrow\left(\operatorname{gr}_{1} Q_{1}\left(\lambda^{0}+\varepsilon_{\lambda}\right)\right)\right|_{\mathrm{G}},
$$

where the second map is the restriction of a map of $\underline{G}$-modules. By Lemma 4.2.2, the socle filtrations of $Q_{1}\left(\lambda^{0}\right) \otimes L\left(p \omega_{\lambda}\right)$ and $Q_{1}\left(\lambda^{0}\right) \otimes L\left(\pi \omega_{\lambda}\right)$ both induce the 
socle filtration on $\left.Q_{1}\left(\lambda^{0}\right) \otimes L\left(p \omega_{\lambda}\right)\right|_{\mathrm{G}}$. Applying Lemma 4.2.4 to $Q_{1}\left(\lambda^{0}\right) \otimes$ $L\left(\pi \omega_{\lambda}\right)$ and using the description of $Q_{1}\left(\lambda^{0}+\nu\right)$ in [Hum06, Section 13.9], we see that the graded pieces of the socle filtration of $Q_{1}\left(\lambda^{0}\right) \otimes L\left(\pi \omega_{\lambda}\right)$ are $\operatorname{gr}_{k}\left(Q_{1}\left(\lambda^{0}\right)\right) \otimes L\left(\pi \omega_{\lambda}\right)$. We claim that the graded pieces of the socle filtration of $Q_{1}\left(\lambda^{0}\right) \otimes L\left(p \omega_{\lambda}\right)$ are $\operatorname{gr}_{k}\left(Q_{1}\left(\lambda^{0}\right)\right) \otimes L\left(p \omega_{\lambda}\right)$. Taking the tensor product of the socle filtration on $Q_{1}\left(\lambda^{0}\right)$ with $L\left(p \omega_{\lambda}\right)$ gives a semisimple filtration $\mathcal{F}$ (as follows from Steinberg's tensor product theorem). Moreover, since the restrictions $\left.Q_{1}\left(\lambda^{0}\right) \otimes L\left(\pi \omega_{\lambda}\right)\right|_{\mathrm{G}}$ and $\left.Q_{1}\left(\lambda^{0}\right) \otimes L\left(p \omega_{\lambda}\right)\right|_{\mathrm{G}}$ are isomorphic, Lemma 4.2.2 implies that the dimensions of the graded pieces of the socle filtrations of $Q_{1}\left(\lambda^{0}\right) \otimes L\left(\pi \omega_{\lambda}\right)$ and $Q_{1}\left(\lambda^{0}\right) \otimes L\left(p \omega_{\lambda}\right)$ agree. A dimension consideration implies that $\mathcal{F}$ is the socle filtration.

In particular, we have

$$
\operatorname{gr}_{1}\left(Q_{1}\left(\lambda^{0}\right) \otimes L\left(p \omega_{\lambda}\right)\right) \cong \bigoplus_{i}\left(L\left(p \omega_{\lambda}\right) \otimes\left(\operatorname{gr}_{1} Q_{1}\left(\lambda_{i}^{0}\right)\right) \otimes \bigotimes_{j \neq i} L\left(\lambda_{j}^{0}\right)\right) .
$$

The algebraic map $L(\theta) \hookrightarrow \operatorname{gr}_{1}\left(Q_{1}\left(\lambda^{0}\right) \otimes L\left(p \omega_{\lambda}\right)\right)$ factors through the direct summand

$$
L\left(\lambda^{i_{0}}\right) \otimes\left(\operatorname{gr}_{1} Q_{1}\left(\lambda_{i_{0}}^{0}\right)\right) \otimes L\left(p \omega_{\lambda}\right)
$$

by alcove considerations, where $\lambda^{i_{0}} \stackrel{\text { def }}{=} \sum_{i \neq i_{0}} \lambda_{i}$. (Note that $\lambda_{i}=\lambda_{i}^{0}$ and $\theta_{i}=\lambda_{i}$ for all $i \neq i_{0}$.) Additionally, the map $\left.F_{1} \rightarrow \operatorname{gr}_{1} \operatorname{Inj}\left(F_{0}\right) \cong\left(\operatorname{gr}_{1} Q_{1}\left(\lambda^{\prime}\right)\right)\right|_{\mathrm{G}}$ factors through the direct summand

$$
\left.L\left(\lambda^{\prime, i_{0}}\right) \otimes\left(\operatorname{gr}_{1} Q_{1}\left(\lambda_{i_{0}}^{\prime}\right)\right)\right|_{\mathrm{G}},
$$

where $\lambda^{\prime, i_{0}}=\sum_{i \neq i_{0}} \lambda_{i}^{\prime}$ since if $j \neq i_{0}$, then the highest weights of the JordanHölder factors of

$$
\left.\left(\left(\bigotimes_{i \neq j} L\left(\lambda_{i}^{\prime}\right)\right) \otimes\left(\operatorname{gr}_{1} Q_{1}\left(\lambda_{j}^{\prime}\right)\right)\right)\right|_{\mathrm{G}}
$$

lie in the same alcove as the highest weight of $L\left(\lambda^{0}\right)$ except exactly at embedding $j$. Thus, it suffices to show that the composition

$$
\begin{aligned}
\left.L\left(\theta^{0}+\varepsilon_{\theta}\right)\right|_{\mathrm{G}} & \left.\left.\hookrightarrow L(\theta)\right|_{\mathrm{G}} \hookrightarrow L\left(\lambda^{i_{0}}\right) \otimes\left(\operatorname{gr}_{1} Q_{1}\left(\lambda_{i_{0}}^{0}\right)\right) \otimes L\left(p \omega_{\lambda}\right)\right|_{\mathrm{G}} \\
& \left.\rightarrow L\left(\lambda^{\prime, i_{0}}\right) \otimes\left(\operatorname{gr}_{1} Q_{1}\left(\lambda_{i_{0}}^{\prime}\right)\right)\right|_{\mathrm{G}}
\end{aligned}
$$

is nonzero.

The $G$-module $\operatorname{gr}_{1} Q_{1}\left(\lambda_{i_{0}}^{0}\right)$ is the direct sum of three irreducible modules in alcoves $A, C$, and $D$ (respectively $B, E$, and $F$ ) if $\lambda_{i_{0}}^{0}$ is in alcove $B$ (respectively $A$ ). Let $\widetilde{w}_{1}, \widetilde{w}_{2}$, and $\widetilde{w}_{3}$ be the elements of $W_{a}$ such that $\widetilde{w}_{1} \cdot A, \widetilde{w}_{2} \cdot A$, and $\widetilde{w}_{3} \cdot A$ are the alcoves $A, C$, and $D$ (respectively $B, E$, and $F$ ), respectively. Let $\widetilde{w}_{B} \in W_{a}$ be 
the element such that $B=\widetilde{w}_{B} \cdot A$. The natural identification $X^{*}(T) \backslash \widetilde{W} / \Omega \cong\{A$, $B$ \} where $\Omega=\operatorname{Stab}_{\widetilde{W}}(A)$ and the fact that $\theta_{i_{0}}^{0}$ is in alcove $A$ (respectively $B$ ) if $\lambda_{i_{0}}^{0}$ is in alcove $B$ (respectively $A$ ) shows that $X^{*}(T) \widetilde{y}_{i_{0}} \widetilde{w}_{B} \Omega=X^{*}(T) \widetilde{w}_{j} \Omega$ for any $j \in\{1,2,3\}$. Moreover, it is not difficult to check that

$$
X^{*}(T) \widetilde{y}_{i_{0}} \widetilde{w}_{B} \Omega=\coprod_{j=1}^{3} X^{*}(T) \widetilde{w}_{j}
$$

This implies that there exist unique $j \in\{1,2,3\}$ and $\omega \in X^{*}(T)$ such that

$$
t_{-\omega_{\theta, i_{0}}} \widetilde{y}_{i_{0}} \widetilde{w}_{B}\left(\widetilde{w}_{\lambda} t_{-\omega_{\lambda, i_{0}}} \widetilde{w}_{i_{0}} \widetilde{w}_{B}\right)^{-1}=t_{-\omega} \widetilde{w}_{j}
$$

where $\tilde{w}_{\lambda} \in W_{a}$ is the unique element such that $\widetilde{w}_{\lambda} \cdot \lambda_{i_{0}}^{0} \in A$ or, equivalently, that $\widetilde{w}_{\lambda} t_{-\omega_{\lambda, i_{0}}} \widetilde{w}_{i_{0}} \widetilde{w}_{B} \in \Omega$. Then we have that

$$
\theta_{i_{0}}^{0}+p \omega=t_{\omega} t_{-\omega_{\theta, i_{0}}} \tilde{y}_{i_{0}} \cdot \mu_{i_{0}}=\widetilde{w}_{j} \widetilde{w}_{\lambda} t_{-\omega_{\lambda, i_{0}}} \widetilde{w}_{i_{0}} \cdot \mu_{i_{0}}=\widetilde{w}_{j} \widetilde{w}_{\lambda} \cdot \lambda_{i_{0}}^{0}
$$

so that, by construction, $\omega \in X^{*}(T)$ is the unique weight such that $L\left(\theta_{i_{0}}^{0}+p \omega\right)$ is a Jordan-Hölder factor of $\operatorname{gr}_{1} Q_{1}\left(\lambda_{i_{0}}^{0}\right)$. We now consider $\omega$ as an element of $X^{*}(\underline{T})$ in the $i_{0}$-embedding.

We now proceed casewise. If $\omega_{\lambda}$ (respectively $\omega_{\theta}$ ) is 0 , then $\operatorname{gr}_{0}\left(\left.M\right|_{\mathrm{G}}\right)$ (respectively $\operatorname{gr}_{1}\left(\left.M\right|_{\mathrm{G}}\right)$ ) is irreducible, and so the map $\left.M\right|_{\mathrm{G}} \rightarrow \operatorname{Inj}\left(F_{0}\right)$ is injective (respectively the map $\left.\operatorname{Proj}\left(F_{1}\right) \rightarrow M\right|_{\mathrm{G}}$ is surjective) and the composition (4.15) is nonzero.

Now assume that $\omega_{\lambda}$ and $\omega_{\theta}$ are both nonzero. At most one of $\omega_{\lambda}$ and $\omega_{\theta}$ can be $\eta_{i_{0}}^{\prime}$. By duality, we can and will assume that $\omega_{\lambda} \neq \eta_{i_{0}}^{\prime}$. Then $\omega_{\lambda}$ is either $\varepsilon_{1, i_{0}}^{\prime}$ or $\varepsilon_{2, i_{0}}^{\prime}$. We assume that $\omega_{\lambda}$ is $\varepsilon_{2, i_{0}}^{\prime}$, the other case being symmetric. So if $\lambda_{i_{0}}$ is in alcove $D$ (respectively $F$ ), then $\theta_{i_{0}}$ is in alcove $E$ or $F$ (respectively $C, D$, or $G$ ) by Proposition 4.2.9. We claim that if $\omega_{\theta}$ is $\varepsilon_{1, i_{0}}^{\prime}$ (respectively $\varepsilon_{2, i_{0}}^{\prime}$ or $\eta_{i_{0}}^{\prime}$ ), then the $\omega$ defined above is $\varepsilon_{2, i_{0}}^{\prime}+(1,1,1)_{i_{0}}$ (respectively 0 or $\varepsilon_{1, i_{0}}^{\prime}$ ). Indeed, since $t_{-\omega} \widetilde{w}_{j} \cdot A \in\{A, B\}$ by (4.18) and $\widetilde{w}_{j} \cdot A \in\{A, B, C, D, E, F\}$ by definition, $\omega_{i_{0}}$ is in $\left\{0, \varepsilon_{1}^{\prime}, \varepsilon_{2}^{\prime}\right\}+X^{0}(T)$. From (4.18), we see that $\omega \equiv \omega_{\theta}-\omega_{\lambda}\left(\bmod \underline{\Lambda}_{R}\right)$. These two facts determine $\omega$.

A morphism

$$
L\left(\theta_{i_{0}}\right) \cong L\left(\theta_{i_{0}}^{0}+p \omega_{\theta}\right) \rightarrow\left(\operatorname{gr}_{1} Q_{1}\left(\lambda_{i_{0}}^{0}\right)\right) \otimes L\left(p \omega_{\lambda}\right)
$$

must factor through $L\left(\theta_{i_{0}}^{0}+p \omega^{\prime}\right) \otimes L\left(p \omega_{\lambda}\right)$ for some $\omega^{\prime}$. By construction, $\omega^{\prime}$ must be $\omega$. We conclude that the map

$$
L(\theta) \hookrightarrow L\left(\lambda^{i_{0}}\right) \otimes\left(\operatorname{gr}_{1} Q_{1}\left(\lambda_{i_{0}}^{0}\right)\right) \otimes L\left(p \omega_{\lambda}\right)
$$


factors through $L\left(\theta^{0}+p \omega\right) \otimes L\left(p \omega_{\lambda}\right)$. It suffices to show that the composition

$$
\begin{aligned}
\left.L\left(\theta^{0}+\varepsilon_{\theta}\right)\right|_{\mathrm{G}} & \left.\left.\hookrightarrow L(\theta)\right|_{\mathrm{G}} \hookrightarrow L\left(\theta^{0}\right) \otimes L(p \omega) \otimes L\left(p \omega_{\lambda}\right)\right|_{\mathrm{G}} \\
& \left.\rightarrow L\left(\lambda^{\prime, i_{0}}\right) \otimes\left(\mathrm{gr}_{1} Q_{1}\left(\lambda_{i_{0}}^{\prime}\right)\right)\right|_{\mathrm{G}}
\end{aligned}
$$

is nonzero.

Let $\varepsilon_{\lambda}^{\prime} \in L\left(\pi \omega_{\lambda}\right)$ be such that $\lambda^{0}+\varepsilon_{\lambda}$ and $\theta^{0}+\varepsilon_{\lambda}^{\prime}$ are in the same $\underline{\widetilde{W}}$-orbit under the $p$-dot action. Similar to an earlier argument of the three simple JordanHölder factors of $L\left(\lambda^{\prime}, i_{0}\right) \otimes\left(\mathrm{gr}_{1} Q_{1}\left(\lambda_{i_{0}}^{\prime}\right)\right)$, only the restriction to $\mathrm{G}$ of one of the form $L\left(\theta^{0}+\varepsilon_{\lambda}^{\prime}\right) \otimes L\left(p \omega^{\prime}\right)$ for some $\omega^{\prime}$ contains $F_{1} \cong F\left(\theta^{0}+\varepsilon_{\theta}\right)$ as a JordanHölder factor. By construction, $\omega^{\prime}$ must be $\omega$. It now suffices to show that the composition

$$
\begin{aligned}
\left.L\left(\theta^{0}+\varepsilon_{\theta}\right)\right|_{\mathrm{G}} & \left.\left.\left.\hookrightarrow L\left(\theta^{0}+\pi \omega_{\theta}\right)\right|_{\mathrm{G}} \cong L(\theta)\right|_{\mathrm{G}} \hookrightarrow L\left(\theta^{0}\right) \otimes L(p \omega) \otimes L\left(p \omega_{\lambda}\right)\right|_{\mathrm{G}} \\
& \left.\left.\cong L\left(\theta^{0}\right) \otimes L\left(\pi \omega_{\lambda}\right) \otimes L(p \omega)\right|_{\mathrm{G}} \rightarrow L\left(\theta^{0}+\varepsilon_{\lambda}^{\prime}\right) \otimes L(p \omega)\right|_{\mathrm{G}}
\end{aligned}
$$

is nonzero. Moreover, the multiplicity $\left[\left.L\left(\theta^{0}+\varepsilon_{\lambda}^{\prime}\right) \otimes L(p \omega)\right|_{\mathrm{G}}: F_{1}\right]$ is one.

Assume now that $\omega_{\theta}$ is not $\varepsilon_{1, i_{0}}^{\prime}$. Then using the assumption that $\left[\left.M\right|_{\mathrm{G}}: F_{1}\right]=1$ so that $\varepsilon_{\theta} \neq 0$, one can check that $F_{1}$ appears in $\left.L\left(\theta^{0}\right) \otimes L(p \omega) \otimes L\left(p \omega_{\lambda}\right)\right|_{\mathrm{G}}$ with multiplicity one, and we see that (4.19) is nonzero.

Finally, we assume that $\omega_{\theta}$ is $\varepsilon_{1, i_{0}}^{\prime}$. Let $\varepsilon$ be $\varepsilon_{\theta}-\varepsilon_{\lambda}^{\prime}$ which is in $L(\pi \omega)$ by the composition (4.19). Note that $\varepsilon \not \equiv \varepsilon_{\lambda}^{\prime}\left(\bmod X^{0}(\underline{T})\right)$. The weight $F_{1}$ appears with multiplicity two in $\left.\bigoplus_{v_{\lambda} \in L\left(\pi \omega_{\lambda}\right)} L\left(\theta^{0}+v_{\lambda}\right) \otimes L(p \omega)\right|_{\mathrm{G}}$ and with multiplicity one in each of $\left.L\left(\theta^{0}+\varepsilon_{\lambda}^{\prime}\right) \otimes L(p \omega)\right|_{\mathrm{G}}$ and $\left.L\left(\theta^{0}+\varepsilon-\pi(1,1,1)_{i_{0}}\right) \otimes L(p \omega)\right|_{\mathrm{G}}$. Assume for the sake of contradiction that the composition (4.19) is zero. Then the image of the composition of the first four maps of (4.19) is contained in $L\left(\theta^{0}+\varepsilon-\pi(1\right.$, $\left.1,1)_{i_{0}}\right)\left.\otimes L(p \omega)\right|_{\mathrm{G}}$. In particular, since $\varepsilon \not \equiv \varepsilon_{\lambda}^{\prime}\left(\bmod X^{0}(\underline{T})\right)$, the image of the composition of the first three maps of (4.19) is not stable under the involution action on $\left.\left.L\left(\theta^{0}\right) \otimes L(p \omega) \otimes L\left(p \omega_{\lambda}\right)\right|_{\mathrm{G}} \cong L\left(\theta^{0}+p(1,1,1)_{i_{0}}\right) \otimes L\left(p \omega_{\lambda}\right) \otimes L\left(p \omega_{\lambda}\right)\right|_{\mathrm{G}}$ which permutes the last two tensor factors. Since $F_{1}$ appears with multiplicity one in $\left.L(\theta)\right|_{\mathrm{G}}$, this implies that the image of

$$
\begin{aligned}
& L\left(\theta^{0}+p(1,1,1)_{i_{0}}\right) \otimes L\left(p \omega_{\theta}-p(1,1,1)_{i_{0}}\right) \\
& \quad \hookrightarrow L\left(\theta^{0}+p(1,1,1)_{i_{0}}\right) \otimes L\left(p \omega_{\lambda}\right) \otimes L\left(p \omega_{\lambda}\right),
\end{aligned}
$$

which induces the second map of (4.19), is not stable under this involution action (note the key role played by Lemma 4.2.11). However, the unique submodule of $L\left(p \omega_{\lambda}\right) \otimes L\left(p \omega_{\lambda}\right)$ isomorphic to $L\left(p \omega_{\theta}-p(1,1,1)_{i_{0}}\right)$ is the submodule where this involution acts by -1 . This is a contradiction. 
Proof of Proposition 4.2.10. We write $\lambda$ and $\theta$ as $\lambda^{0}+p \omega_{\lambda}$ and $\theta^{0}+p \omega_{\theta}$, respectively, where $\lambda^{0}$ and $\theta^{0}$ are $p$-restricted weights. Write $\omega_{\lambda}$ as $\omega_{\lambda, i_{0}}+\omega_{\lambda}^{i_{0}}$ where $\omega_{\lambda, i_{0}}$ (respectively $\omega_{\lambda}^{i_{0}}$ ) is 0 away from (respectively at) embedding $i_{0}$. Similarly, write $\omega_{\theta}$ as $\omega_{\theta, i_{0}}+\omega_{\theta}^{i_{0}}$. Then $\omega_{\lambda}^{i_{0}}$ equals $\omega_{\theta}^{i_{0}}$ by assumption, and so we set $\omega^{i_{0}} \stackrel{\text { def }}{=} \omega_{\lambda}^{i_{0}}=\omega_{\theta}^{i_{0}}$. Let $M_{i_{0}}$ be the unique up to isomorphism nontrivial extension of $L\left(\theta_{i_{0}}\right)$ by $L\left(\lambda_{i_{0}}\right)$ and let $M^{i_{0}}$ be $\bigotimes_{i \neq i_{0}} L\left(\lambda_{i}\right)$ so that $M$ is isomorphic to $M_{i_{0}} \otimes M^{i_{0}}$. Then $\left.M\right|_{\mathrm{G}}$ is isomorphic to

$$
\begin{aligned}
\left.M_{i_{0}} \otimes M^{i_{0}}\right|_{\mathrm{G}} & \left.\cong M_{i_{0}} \otimes\left(\bigotimes_{i \neq i_{0}} L\left(\lambda_{i}^{0}\right)\right) \otimes L\left(p \omega^{i_{0}}\right)\right|_{\mathrm{G}} \\
& \left.\cong M_{i_{0}} \otimes\left(\bigotimes_{i \neq i_{0}} L\left(\lambda_{i}^{0}\right)\right) \otimes L\left(\pi \omega^{i_{0}}\right)\right|_{\mathrm{G}}
\end{aligned}
$$

Let $M^{\prime}$ be $M_{i_{0}} \otimes\left(\bigotimes_{i \neq i_{0}} L\left(\lambda_{i}^{0}\right)\right) \otimes L\left(\pi \omega^{i_{0}}\right)$. By Lemma 4.2.2, we have that $\left.\left.\operatorname{soc}\left(M^{\prime}\right)\right|_{\mathrm{G}} \cong \operatorname{soc}\left(\left.M^{\prime}\right|_{\mathrm{G}}\right) \cong \operatorname{soc}\left(\left.M\right|_{\mathrm{G}}\right) \cong \operatorname{soc}(M)\right|_{\mathrm{G}}$ so that

$$
\operatorname{soc}\left(M^{\prime}\right) \cong L\left(\lambda_{i_{0}}\right) \otimes\left(\bigotimes_{i \neq i_{0}} L\left(\lambda_{i}^{0}\right)\right) \otimes L\left(\pi \omega^{i_{0}}\right) .
$$

Similarly, we have that

$$
\operatorname{cosoc}\left(M^{\prime}\right) \cong L\left(\theta_{i_{0}}\right) \otimes\left(\bigotimes_{i \neq i_{0}} L\left(\lambda_{i}^{0}\right)\right) \otimes L\left(\pi \omega^{i_{0}}\right) .
$$

The socle and cosocle of $M^{\prime}$ can be decomposed using Lemma 4.2.4. Fix a direct sum decomposition $\operatorname{cosoc}\left(M^{\prime}\right)=\bigoplus_{j} M_{1, j}^{\prime}$ into simple modules. Using the known dimensions of $\operatorname{Ext}_{\underline{G}}^{1}$ groups between simple modules and the fact that $M^{\prime}$ is rigid of Loewy length 2 , one sees that the minimal submodule $M_{j}^{\prime}$ of $M^{\prime}$ whose projection to $\operatorname{cosoc}\left(M^{\prime}\right)$ contains $M_{1, j}^{\prime}$ has length exactly 2 . Thus, the natural surjection $\oplus M_{j}^{\prime} \rightarrow \sum M_{j}^{\prime}=M^{\prime}$ is an isomorphism by length considerations. More explicitly, if $M_{1, j}^{\prime}$ is isomorphic to $L\left(\lambda^{0}+p \omega_{\lambda, i_{0}}+\pi \varepsilon^{i_{0}}\right)$ for some $\varepsilon^{i_{0}} \in L\left(\omega^{i_{0}}\right)$, then $M_{0, j}^{\prime}$ is isomorphic to $L\left(\theta^{0}+p \omega_{\theta, i_{0}}+w \pi \varepsilon^{i_{0}}\right)$ for the unique element $w \in \underline{W}$ such that these weights are linked.

The alcoves of $F_{1}$ and $F_{0}$ differ only in embedding $i_{0}$. Then by Lemma 4.2.6, the corresponding elements of $\underline{\Lambda}_{W}^{v}$ under $\mathfrak{T r}_{v}$ for any appropriate $v$ must be the same for all embeddings except for $\pi\left(i_{0}\right)$. By the above explicit description, we conclude that $F_{1}$ is in $\mathrm{JH}\left(\left.M_{1, j}^{\prime}\right|_{\mathrm{G}}\right)$ if and only if $F_{0}$ is in $\mathrm{JH}\left(\left.M_{0, j}^{\prime}\right|_{\mathrm{G}}\right)$. So it suffices to prove the proposition for $M_{j}^{\prime}$ in place of $M$. This now follows from Proposition 4.2.12. 
Proof of Theorem 4.2.7(3). Let $\Gamma$ be the directed graph with vertices $\mathrm{JH}(U)=$ $\mathrm{JH}\left(\left.V_{\mu}\right|_{\mathrm{G}}\right)$ so that there is a directed edge $\sigma_{1} \rightarrow \sigma_{2}$ for $\sigma_{1}$ and $\sigma_{2} \in \mathrm{JH}\left(\left.V_{\mu}\right|_{\mathrm{G}}\right)$ if and only if $\mathrm{d}_{\mathrm{gph}}\left(\sigma_{(0,1)}, \sigma_{1}\right)>\mathrm{d}_{\mathrm{gph}}\left(\sigma_{(0,1)}, \sigma_{2}\right)$ and $\mathrm{d}_{\mathrm{gph}}\left(\sigma_{1}, \sigma_{2}\right)=1$. Note that the first condition ensures that $\Gamma$ is acyclic.

We claim that $\Gamma$ is a subgraph of $\Gamma(U)$. Let $\sigma_{1}$ and $\sigma_{2}$ be in $\operatorname{JH}(U)$ with $\mathrm{d}_{\mathrm{gph}}\left(\sigma_{1}, \sigma_{2}\right)=1$. Let $d_{j}=\mathrm{d}_{\mathrm{gph}}\left(\sigma_{(0, \underline{1})}, \sigma_{j}\right)$ for $j=1$ and 2 and suppose that $d_{1}>d_{2}$ so that $d_{1}=d_{2}+1$. Then by Lemma 4.2.8, $d_{U}^{\text {soc }}\left(\sigma_{j}\right)$ is $d_{j}$ for $j=1$ and 2. Moreover, by Theorem 4.2.7(2) and parity reasons, $\left[\left.\operatorname{gr}_{d_{j}}\left(V_{\mu}\right)\right|_{\mathrm{G}}: \sigma_{k}\right]=\delta_{j k}$ for $j$ and $k$ in $\{1,2\}$. Using that every nontrivial extension which can occur in the layers of $V_{\mu}$ does occur (see Table 6), it is easy to check that there is a unique length 2 subquotient $M$ of $\operatorname{Fil}_{d_{1}}\left(V_{\mu}\right) / \operatorname{Fil}_{d_{2}-1}\left(V_{\mu}\right)$ such that $\sigma_{1}$ and $\sigma_{2}$ appear in $\mathrm{JH}\left(\left.M\right|_{\mathrm{G}}\right)$ with multiplicity one. By Proposition 4.2.10, there is a subquotient of $\left.M\right|_{\mathrm{G}}$ which is a nonsplit extension of $\sigma_{1}$ by $\sigma_{2}$. For multiplicity reasons, this must also be a subquotient of $U$. Hence, there is a directed edge from $\sigma_{1}$ to $\sigma_{2}$ in $\Gamma(U)$.

We now claim that if $\Gamma$ is a subgraph of a directed graph $\Gamma^{\prime}$ such that

(1) $\Gamma$ and $\Gamma^{\prime}$ have the same vertices,

(2) $\Gamma^{\prime}$ is acyclic, and

(3) $\sigma_{1} \rightarrow \sigma_{2}$ is a subgraph of $\Gamma^{\prime}$ only if $\mathrm{d}_{\mathrm{gph}}\left(\sigma_{1}, \sigma_{2}\right)=1$,

then $\Gamma^{\prime}=\Gamma$. Since $\Gamma(U)$ satisfies these conditions ((3) follows from Lemmas 4.2.5 and 4.2.6), this would complete the proof.

Assume that $\sigma_{1} \rightarrow \sigma_{2}$ is a subgraph of $\Gamma^{\prime}$. By (3) and the geometry of the extension graph, we have that $\mathrm{d}_{\mathrm{gph}}\left(\sigma_{(0,1)}, \sigma_{1}\right)=\mathrm{d}_{\mathrm{gph}}\left(\sigma_{(0,1)}, \sigma_{2}\right) \pm 1$, and, hence, it is enough to prove that $\mathrm{d}_{\mathrm{gph}}\left(\sigma_{(0,1)}, \sigma_{1}\right)=\mathrm{d}_{\mathrm{gph}}\left(\sigma_{(0,1)}, \sigma_{2}\right)+1$. Suppose otherwise. Then $\sigma_{2} \rightarrow \sigma_{1}$ is a subgraph of $\Gamma$ by definition and thus a subgraph of $\Gamma^{\prime}$. But this contradicts (2).

4.2.4. The embedding construction. We start with the following observation.

Lemma 4.2.13. Let $R$ be an $n$-generic Deligne-Lusztig representation. Then $R$ is $n$-2-deep.

Proof. If we write $R=R_{s}(\mu+\eta)$ with $\mu$ being $n$-deep, it is hence enough to prove that $\sigma_{r(\omega, a)}$ is $n-2$-deep for any obvious weight $\sigma_{(\omega, a)}$ in $\mathrm{JH}(\bar{R})$. This follows from Proposition 2.3.4.

From now onward, we assume that $\mu$ is 6-deep in alcove $\underline{B}$. (By Lemma 4.2.13, the Deligne-Lusztig representation $R \stackrel{\text { def }}{=} R_{s}\left(\mu^{\mathrm{op}}+\eta^{\prime}\right)$ is therefore 4-deep.) We write $\sigma^{\text {op }} \stackrel{\text { def }}{=} F(\mu)=F\left(\mathfrak{T r}_{\mu^{\mathrm{op}}+\eta}(0,1)\right)$. Note from Proposition 2.3.5 that the 
unique element $\sigma \in \mathrm{JH}(\bar{R})$ satisfying $\mathrm{d}_{\mathrm{gph}}\left(\sigma, \sigma^{\mathrm{op}}\right)=3 f$ (that is, having maximal graph distance from $\sigma^{\mathrm{op}}$ ) is

$$
\sigma \stackrel{\text { def }}{=} F\left(\mu^{\mathrm{op}}+s\left(\eta^{\prime}\right)\right)=F\left(\mathfrak{T r}_{\mu^{\mathrm{op}}+\eta}\left(s\left(\eta^{\prime}\right), 0\right)\right) .
$$

To ease notation, we write $\lambda \stackrel{\text { def }}{=} \mu^{\text {op }}+s\left(\eta^{\prime}\right)$ (hence, $\sigma=F(\lambda)$ ).

LEMmA 4.2.14. Let $\mu \in X_{1}(\underline{T})$ be a weight such that $\mu \in \underline{B}$ is 6-deep and $R \stackrel{\text { def }}{=} R_{s}\left(\mu^{\text {op }}+\eta^{\prime}\right)$. Set $\sigma^{\text {op }} \stackrel{\text { def }}{=} F(\mu)$ and let $\sigma \stackrel{\text { def }}{=} F(\lambda) \in \mathrm{JH}(\overline{\bar{R}})$ be the unique constituent at maximal graph distance. We have

$$
\left[\operatorname{Inj}\left(\sigma^{\mathrm{op}}\right): \sigma\right]=\left[\left.V_{\mu}\right|_{\mathrm{G}}: \sigma\right]=[U: \sigma]=\left[\mathrm{gr}_{3 f}\left(\left.V_{\mu}\right|_{\mathrm{G}}\right): \sigma\right]=1 .
$$

Proof. The equations $\left[\left.V_{\mu}\right|_{\mathrm{G}}: \sigma\right]=[U: \sigma]=\left[\mathrm{gr}_{3 f}\left(\left.V_{\mu}\right|_{\mathrm{G}}\right): \sigma\right]=1$ follow from Theorem 4.2.7(2). The equation $\left[\operatorname{Inj}\left(\sigma^{\mathrm{op}}\right): \sigma\right]=1$ is proved similarly using Theorem 4.2.1.

Proposition 4.2.15. Let $\mu \in X_{1}(\underline{T)}$ be a weight such that $\mu \in \underline{B}$ is 6-deep and $R \stackrel{\text { def }}{=} R_{s}\left(\mu^{\mathrm{op}}+\eta^{\prime}\right)$. Set $\sigma^{\mathrm{op}} \stackrel{\text { def }}{=} F(\mu)$ and let $\sigma \stackrel{\text { def }}{=} F(\lambda) \in \mathrm{JH}(\bar{R})$ be the unique constituent at maximal graph distance. Let $R^{\sigma}$ be an $\mathcal{O}$-lattice in $R$ with irreducible cosocle isomorphic to $\sigma$. There is an injection $\iota: \bar{R}^{\sigma} \rightarrow U$.

Note that the map $\iota$ is unique up to scalar as there is a unique up to scalar nonzero morphism Proj $(\sigma) \rightarrow U$ by Lemma 4.2.14.

Proof. Since $\sigma^{\mathrm{op}} \in \mathrm{JH}\left(\bar{R}^{\sigma}\right)$, there is a unique up to scalar nonzero map

$$
\bar{R}^{\sigma} \rightarrow \operatorname{Inj}\left(\sigma^{\text {op }}\right) \text {. }
$$

We first prove that the map (4.20) factors through the embedding

$$
U \stackrel{\alpha}{\hookrightarrow} \operatorname{Inj}\left(\sigma^{\text {op }}\right)
$$

or, equivalently, that the composite of (4.20) with the natural projection $\operatorname{Inj}\left(\sigma^{\mathrm{op}}\right) \rightarrow \operatorname{coker}(\alpha)$ is zero. As formation of cosocle is right exact and $\bar{R}^{\sigma}$ has irreducible cosocle isomorphic to $\sigma$, the image of the composite map $\bar{R}^{\sigma} \rightarrow \operatorname{coker}(\alpha)$ is either zero or has irreducible cosocle $\sigma$. By Lemma 4.2.14, we know that $\sigma$ is not a Jordan-Hölder constituent of $\operatorname{coker}(\alpha)$, and, therefore, $\operatorname{Hom}_{\mathrm{G}}\left(\bar{R}^{\sigma}, \operatorname{coker}(\alpha)\right)=0$. Now the image of this nonzero map contains $\sigma$ as a Jordan-Hölder factor. Since the minimal submodule of $U$ containing $\sigma$ as a Jordan-Hölder factor has length $9^{f}$, which is the length of $\bar{R}^{\sigma}$, by Theorem 4.2.7(3) and Proposition 4.1.1, this map must be an injection. 
THEOREM 4.2.16. With the hypotheses of Theorem 4.1.9, assume, moreover, that $\sigma$ is a lowest alcove weight with $\operatorname{Def}_{R}(\sigma)=0$. Then Theorem 4.1.9(1)-(4) hold.

Proof. By Remark 4.1.10, it suffices to prove Theorem 4.1.9(3). This follows from Theorem 4.2.7(3) and Propositions 4.2.15 and 4.1.2.

4.3. Proof of the structure theorem in the general case. The aim of this section is to deduce Theorem 4.1.9 from the particular case in Theorem 4.2.16. For this, we introduce a third notion of distance called the saturation distance (see Definition 4.3.4). The first step is to show an inequality between the graph and saturation distances (Corollary 4.3.8) and then proceed further in Section 4.3.2 to prove that under appropriate conditions on $\sigma \in \mathrm{JH}(\bar{R})$ (that is, when $\sigma$ is maximally saturated), the notions of graph, saturation, and cosocle distances $\left(\mathrm{d}_{\mathrm{rad}}^{\sigma}(\kappa)\right)$ actually coincide (Proposition 4.3.16). The agreement of the three distances is equivalent to Theorem 4.1.9, taking into account Lemma 4.3.12. In Section 4.3.3, we show that all weights are maximally saturated, thus concluding the proof of Theorem 4.1.9.

4.3.1. Notions of distances. In what follows, $R$ is a 3-generic Deligne-Lusztig representation. Recall that if $\sigma \in \mathrm{JH}(\bar{R})$, we write $\mathrm{d}_{\mathrm{rad}}^{\sigma}$ as a shorthand for $\mathrm{d}_{\mathrm{rad}}^{\bar{R}^{\sigma}}$. We establish some properties of $\mathrm{d}_{\mathrm{rad}}^{\sigma}$. We deduce the following from Lemma 4.2.6.

Corollary 4.3.1. Assume that $\bar{R}$ is 6-deep and $\sigma \in \mathrm{JH}(\bar{R})$. Then $\mathrm{d}_{\mathrm{gph}}(\sigma$, $\left.\sigma^{\prime}\right) \leqslant \mathrm{d}_{\mathrm{rad}}^{\sigma}\left(\sigma^{\prime}\right)$ for all $\sigma^{\prime} \in \mathrm{JH}(\bar{R})$.

Proof. The assumption that $\bar{R}$ is 6-deep implies that all the elements in $\mathrm{JH}(\bar{R})$ satisfy the hypotheses of Lemma 4.2.6. If $\kappa \in \mathrm{JH}(\bar{R})$ and $k+1=\mathrm{d}_{\text {rad }}^{\sigma}(\kappa)$, then $\operatorname{Ext}_{\mathrm{G}}^{1}\left(\operatorname{gr}^{k}\left(\bar{R}^{\sigma}\right), \kappa\right) \neq 0$. Hence, $\operatorname{Ext}_{\mathrm{G}}^{1}\left(\kappa^{\prime}, \kappa\right) \neq 0$ for some $\kappa^{\prime} \in \mathrm{JH}(\bar{R})$ with $\mathrm{d}_{\mathrm{rad}}^{\sigma}\left(\kappa^{\prime}\right)=k$. Equivalently, $\mathrm{d}_{\mathrm{gph}}\left(\kappa, \kappa^{\prime}\right)=1$ by Lemma 4.2.6. If $d=\mathrm{d}_{\mathrm{rad}}^{\sigma}\left(\sigma^{\prime}\right)$, we conclude that there is a sequence of weights $\sigma_{i}$ for $0 \leqslant i \leqslant d-1$ such that $\sigma_{0}=\sigma, \sigma_{d}=\sigma^{\prime}$ and $\mathrm{d}_{\mathrm{gph}}\left(\sigma_{i}, \sigma_{i+1}\right)=1$ for all $i$. Hence, $\mathrm{d}_{\mathrm{gph}}\left(\sigma, \sigma^{\prime}\right) \leqslant d$.

LEMMA 4.3.2. Let $\sigma, \sigma_{1} \in \mathrm{JH}(\bar{R})$. There is a unique minimal subrepresentation $Q_{\sigma}\left(\sigma_{1}\right) \subseteq \bar{R}^{\sigma}$ containing $\sigma_{1}$ as a Jordan-Hölder factor. Moreover, we have that

(1) $\operatorname{cosoc}\left(Q_{\sigma}\left(\sigma_{1}\right)\right) \cong \sigma_{1}$;

(2) $Q_{\sigma}\left(\sigma_{1}\right)$ is the image of any nonzero map $\bar{R}^{\sigma_{1}} \rightarrow \bar{R}^{\sigma}$. 
Proof. Let $Q_{\sigma}\left(\sigma_{1}\right)$ be the image of any nonzero map $\bar{R}^{\sigma_{1}} \rightarrow \bar{R}^{\sigma}$. It is clear that $\operatorname{cosoc}\left(Q_{\sigma}\left(\sigma_{1}\right)\right) \cong \sigma_{1}$. Suppose that $M \subset \bar{R}^{\sigma}$ is a subrepresentation containing $\sigma_{1}$ as a Jordan-Hölder factor. As $\bar{R}^{\sigma}$ is multiplicity free, $\bar{R}^{\sigma} / M$ does not contain $\sigma_{1}$ as a Jordan-Hölder factor, and, hence, the composition $Q_{\sigma}\left(\sigma_{1}\right) \hookrightarrow \bar{R}^{\sigma} \rightarrow \bar{R}^{\sigma} / M$ is 0 or, equivalently, $Q_{\sigma}\left(\sigma_{1}\right) \subset M$.

LEMMA 4.3.3. Let $\sigma$ and $\sigma_{1} \in \mathrm{JH}(\bar{R})$ and let $\sigma_{2} \in \mathrm{JH}\left(Q_{\sigma}\left(\sigma_{1}\right)\right)$. If $n=\mathrm{d}_{\mathrm{rad}}^{\sigma_{1}}\left(\sigma_{2}\right)$, then we have $\operatorname{Hom}_{\mathrm{G}}\left(\sigma_{2}, \operatorname{gr}_{\mathrm{rad}}^{n}\left(Q_{\sigma}\left(\sigma_{1}\right)\right)\right) \neq 0$. Moreover, $\mathrm{d}_{\mathrm{rad}}^{\sigma}\left(\sigma_{2}\right) \geqslant \mathrm{d}_{\mathrm{rad}}^{\sigma}\left(\sigma_{1}\right)+$ $\mathrm{d}_{\mathrm{rad}}^{\sigma_{1}}\left(\sigma_{2}\right)$.

Note that, a priori, the inequality may be strict so that what we call the cosocle distance $\mathrm{d}_{\mathrm{rad}}^{\sigma}(\kappa)$ from $\sigma$ to $\kappa$ is not a priori necessarily a metric.

Proof. Since formation of cosocle is right exact, we see that the map $\bar{R}^{\sigma_{1}} \rightarrow$ $Q_{\sigma}\left(\sigma_{1}\right)$ is strictly compatible with the radical filtrations. This proves the first claim.

Let $\mathscr{F}=\left\{\mathrm{Fil}^{k}\left(\bar{R}^{\sigma}\right)\right\}_{k}$ be the decreasing filtration defined by $\mathrm{Fil}^{k}\left(\bar{R}^{\sigma}\right)=$ $\operatorname{rad}^{k+\mathrm{d}_{\mathrm{rad}}^{\sigma}\left(\sigma_{1}\right)}\left(\bar{R}^{\sigma}\right)$ (that is, $\mathscr{F}$ is the radical filtration of $\bar{R}^{\sigma}$ shifted by $\mathrm{d}_{\mathrm{rad}}^{\sigma}\left(\sigma_{1}\right)$ ). Under the inclusion $Q_{\sigma}\left(\sigma_{1}\right) \hookrightarrow \bar{R}^{\sigma}$, $\mathscr{F}$ induces a semisimple filtration on $Q_{\sigma}\left(\sigma_{1}\right)$, beginning at 0 . So, with the radical filtration on the domain and $\mathscr{F}$ on the codomain, this inclusion is compatible with the filtrations. This immediately gives $\mathrm{d}_{\mathrm{rad}}^{\sigma}\left(\sigma_{2}\right)-\mathrm{d}_{\mathrm{rad}}^{\sigma}\left(\sigma_{1}\right) \geqslant \mathrm{d}_{\mathrm{rad}}^{\sigma_{1}}\left(\sigma_{2}\right)$ for any $\sigma_{2} \in \mathrm{JH}\left(Q_{\sigma}\left(\sigma_{1}\right)\right)$.

We now introduce a third notion of distance on the set $\mathrm{JH}(\bar{R})$ and give a comparison result with the graph distance (Corollary 4.3.8). To do this, we make crucial use of a global input (Proposition 4.3.7). We also use these results to deduce some basic results about $\Gamma_{\text {rad }}\left(\bar{R}^{\sigma}\right)$ (see, for example, Lemma 4.3.12).

Definition 4.3.4. Let $\sigma_{1}, \sigma_{2} \in \mathrm{JH}(\bar{R})$. Let $R^{\sigma_{1}}$ be an $\mathcal{O}$-lattice with irreducible cosocle $\sigma_{1}$ and fix a saturated inclusion of lattices $R^{\sigma_{2}} \subseteq R^{\sigma_{1}}$. The saturation distance (with respect to $R$ ) between $\sigma_{1}$ and $\sigma_{2}$, noted by $\mathrm{d}_{\text {sat }}\left(\sigma_{1}, \sigma_{2}\right)$, is defined to be the unique integer $d$ such that $p^{d} R^{\sigma_{1}} \subseteq R^{\sigma_{2}}$ is a saturated inclusion.

REMARK 4.3.5. We indicate a justification for the existence of the integer $d$ in Definition 4.3.4. Given two $\mathcal{O}$-lattices $\Lambda_{1}$ and $\Lambda_{2}$ in an $E$-vector space $V$, there is a unique $d \in \mathbb{Z}$ such that $\varpi^{d} \Lambda_{2} \subset \Lambda_{1}$ is a saturated inclusion. If $V, \Lambda_{1}$, and $\Lambda_{2}$ are obtained from base change from an unramified subfield of $E$, then, in fact, $\varpi^{d}$ is a power of $p$ up to units. Since $R, R^{\sigma_{1}}$, and $R^{\sigma_{2}}$ are defined over an unramified extension of $\mathbb{Q}_{p}$, the integer $d$ in Definition 4.3.4 exists.

The following lemma is clear. 
LEMMA 4.3.6. The saturation distance is a metric on $\mathrm{JH}(\bar{R})$.

The following proposition is the main global input.

Proposition 4.3.7. Let $R$ be a 13-generic Deligne-Lusztig representation. Let $\sigma_{1}, \sigma_{2} \in \mathrm{JH}(\bar{R})$ be such that $\mathrm{d}_{\mathrm{gph}}\left(\sigma_{1}, \sigma_{2}\right)=1$. Then $\mathrm{d}_{\mathrm{sat}}\left(\sigma_{1}, \sigma_{2}\right)=1$.

Proof. Let $\tau_{\mathcal{S}}$ be the collection of tame inertial type such that $\sigma\left(\tau_{\mathcal{S}}\right)=R$. We can and do fix a collection $\bar{\rho}_{\mathcal{S}}$ of semisimple 10-generic Galois representation such that if $\widetilde{w} \stackrel{\text { def }}{=} \widetilde{w}\left(\bar{\rho}_{\mathcal{S}}, \tau_{\mathcal{S}}\right)$, then $\ell\left(\widetilde{w}_{i}^{*}\right) \geqslant 2$ for all $i \in \mathcal{J}$ and $\sigma_{1}$ and $\sigma_{2} \in W^{?}\left(\bar{\rho}_{\mathcal{S}}\right.$, $\tau_{\mathcal{S}}$ ). (Note that the condition that $R$, hence $\tau_{\mathcal{S}}$, is 13-generic guarantees that $\bar{\rho}_{\mathcal{S}}$ is 10 -generic by the argument of Proposition 3.4.5.) Fix a weak minimal patching functor $M_{\infty}$ for $\bar{\rho}_{\mathcal{S}}$, which exists by Corollary 3.5.16. Recall $R_{\overline{\mathfrak{M}}_{\tilde{v}}, \bar{\rho}_{\widetilde{v}}}^{\tau_{\tilde{v}}, \square}$ from [LLHLM18, Definition 5.10].

For $i \in\{1,2\}$, define the modules

$$
\begin{array}{r}
M_{\infty}^{\prime}\left(R^{\sigma_{i}}\right) \stackrel{\text { def }}{=} M_{\infty}\left(R^{\sigma_{i}}\right) \widehat{\otimes}_{\left(\widehat{\otimes}_{\tilde{v} \in \mathcal{S}} R_{\overline{\rho_{v}}}^{\square}\right)}\left(\widehat{\bigotimes}_{\widehat{\bigotimes}_{\infty} \in \mathcal{S}} R_{\overline{\mathfrak{M}}_{\tilde{v}}, \bar{\rho}_{\widetilde{v}}}^{\bar{\tau}_{\widetilde{v}}, \square}\right) \\
M_{\infty}^{\prime}\left(\sigma_{i}\right) \stackrel{\text { def }}{=} M_{\infty}\left(\sigma_{i}\right) \widehat{\otimes}_{\left(\widehat{\otimes}_{\tilde{v} \in \mathcal{S}} R_{\widetilde{\rho_{\tilde{v}}}}^{\square}\right)}\left(\widehat{\bigotimes}_{\widetilde{v} \in \mathcal{S}} R_{\overline{\mathfrak{M}}_{\tilde{v}}, \bar{\beta}_{\widetilde{v}}, \square}\right) .
\end{array}
$$

Similarly, define $R_{\infty}^{\prime}\left(\tau_{\mathcal{S}}\right)$ and $\bar{R}_{\infty}^{\prime}\left(\tau_{\mathcal{S}}\right)$. Then $M_{\infty}^{\prime}\left(R^{\sigma_{i}}\right)$ is $p$-torsion-free and maximally Cohen-Macaulay over $R_{\infty}^{\prime}\left(\tau_{\mathcal{S}}\right)$, and generically of rank one, and similarly $M_{\infty}^{\prime}\left(\sigma_{i}\right)$ is maximally Cohen-Macaulay over $\bar{R}_{\infty}^{\prime}\left(\tau_{\mathcal{S}}\right)$.

We recall the setup of Lemma 3.6.10. The ring $R_{\infty}^{\prime}\left(\tau_{\mathcal{S}}\right)$ is formally smooth over $\widehat{\bigotimes}_{\widetilde{v} \in \mathcal{S}} R_{\overline{\mathfrak{M}}_{\widetilde{v}}, \widetilde{w}_{\widetilde{v}}}^{\operatorname{exl}, \nabla}$, where we write $\overline{\mathfrak{M}}_{\widetilde{v}} \in Y^{\eta, \tau_{\widetilde{v}}}(\mathbb{F})$ for the unique Kisin module corresponding to $\bar{\rho}_{\widetilde{v}}$ as in Theorem 3.3.12. By letting $N \stackrel{\text { def }}{=} \sum_{i \in \mathcal{J}}(4-$ $\left.\ell\left(\widetilde{w}_{i}^{*}\right)\right)$, the latter ring is formally smooth over $R_{N} \stackrel{\text { def }}{=} \widehat{\bigotimes}_{j=1}^{N} \mathcal{O} \llbracket x_{j}, y_{j} \rrbracket /\left(x_{j} y_{j}-\right.$ p) by [LLHLM18, §5.3.2] using that $\ell\left(\widetilde{w}_{i}^{*}\right)>1$ for all $i \in \mathcal{J}$. Fix an isomorphism $R_{N} \llbracket t_{1}, \ldots, t_{k} \rrbracket \stackrel{\sim}{\rightarrow} R_{\infty}^{\prime}\left(\tau_{\mathcal{S}}\right)$. Let $S \subset R_{N} \llbracket t_{1}, \ldots, t_{k} \rrbracket$ be the subring $\mathbb{Z}_{p} \llbracket\left(x_{j}, y_{j}\right)_{j=1}^{N},\left(t_{j}\right)_{j=1}^{k} \rrbracket /\left(x_{j} y_{j}-p\right)_{j=1}^{N}$. If $M^{\prime}$ is a maximal Cohen-Macaulay $R_{\infty}^{\prime}\left(\tau_{\mathcal{S}}\right)$-module, it is a maximal Cohen-Macaulay $S$-module as well by [Gro65, Corollaire 5.7.10] and using that the maximal ideal of $R_{\infty}^{\prime}\left(\tau_{\mathcal{S}}\right)$ is the only prime above the maximal ideal of $S$. The maximality follows from the fact that $R_{\infty}^{\prime}\left(\tau_{\mathcal{S}}\right)$ is finite over $S$. It is convenient to work over $S$ below since Spec $S / p S$ is reduced.

For $\sigma \in \mathrm{JH}(\bar{R}), \operatorname{Ann}_{R_{\infty}^{\prime}\left(\tau_{\mathcal{S}}\right)}\left(M_{\infty}^{\prime}(\sigma)\right)$ is $\mathfrak{p}(\sigma) R_{\infty}^{\prime}\left(\tau_{\mathcal{S}}\right)$ by Proposition 3.6.1(2). For $\sigma \in W^{?}\left(\bar{\rho}_{\mathcal{S}}, \tau_{\mathcal{S}}\right)$, let $\mathfrak{p}(\sigma)$ be $\left(z_{j}(\sigma)\right)_{j=1}^{N}+(\varpi)$ where $z_{j}(\sigma) \in\left\{x_{j}, y_{j}\right\}$ for each $1 \leqslant j \leqslant N$. Let $\mathfrak{p}_{S}(\sigma) \subset S$ be the preimage of $\mathfrak{p}(\sigma) R_{\infty}^{\prime}\left(\tau_{\mathcal{S}}\right)$ for all $\sigma \in$ $\operatorname{JH}(\bar{R})$ so that $\mathfrak{p}_{S}(\sigma)=\left(z_{j}(\sigma)\right)_{j=1}^{N}+(p)$ and $\operatorname{Supp}_{S} M_{\infty}^{\prime}(\sigma)=\operatorname{Spec}\left(S / \mathfrak{p}_{S}(\sigma)\right)$. 
For all $1 \leqslant j \leqslant N$, assume, without loss of generality, that $z_{j}\left(\sigma_{1}\right)=x_{j}$ and let $z_{j} \stackrel{\text { def }}{=} z_{j}\left(\sigma_{2}\right)$. Then by Lemma 3.6.10,\# $\left.\#\left\{x_{j}\right\}_{j} \Delta\left\{z_{j}\right\}_{j}\right)=2$. We assume, without loss of generality, that $z_{1}=y_{1}$ and $z_{j}=x_{j}$ for $j \neq 1$. To simplify notation, let $R_{i}=R^{\sigma_{i}}$ for $i=1,2$. We fix a chain of saturated inclusion of lattices $p^{k} R_{1} \subseteq$ $R_{2} \subseteq R_{1}$ with $k \geqslant 1$. Since $R$ is residually multiplicity free, $C \stackrel{\text { def }}{=} \operatorname{coker}\left(R_{2}+\right.$ $p R_{1} \hookrightarrow R_{1}$ ) does not contain $\sigma_{2}$ as a Jordan-Hölder factor (as can be seen from descent to an unramified coefficient ring). Thus,

$$
\operatorname{Supp}_{S} M_{\infty}^{\prime}(C) \subset \bigcup_{\sigma \in \mathrm{JH}(\bar{R}), \sigma \neq \sigma_{2}} \operatorname{Spec} S / \mathfrak{p}_{S}(\sigma) .
$$

The scheme-theoretic support of $M_{\infty}^{\prime}(C)$ in Spec $S$ is contained in Spec $S / p S$ and is thus generically reduced so that by the proof of Lemma 3.6.2, the schemetheoretic support of $M_{\infty}^{\prime}(C)$ in Spec $S$ is a closed subscheme of

$$
\operatorname{Spec}\left(S / \bigcap_{\sigma \in \mathrm{JH}(\bar{R}), \sigma \neq \sigma_{2}} \mathfrak{p}_{S}(\sigma)\right) \text {. }
$$

Since $x_{1} y_{2} \cdots y_{N} \in \mathfrak{p}_{S}(\sigma)$ for all $\sigma \in \mathrm{JH}(\bar{R})$ with $\sigma \neq \sigma_{2}, x_{1} y_{2} \cdots y_{N}$ annihilates $M_{\infty}^{\prime}(C)$ or, equivalently,

$$
x_{1} y_{2} \cdots y_{N} M_{\infty}^{\prime}\left(R_{1}\right) \subset M_{\infty}^{\prime}\left(R_{2}\right)+p M_{\infty}^{\prime}\left(R_{1}\right) .
$$

Symmetrically, we have

$$
y_{1} y_{2} \cdots y_{N} M_{\infty}^{\prime}\left(R_{2}\right) \subset M_{\infty}^{\prime}\left(p^{k} R_{1}\right)+p M_{\infty}^{\prime}\left(R_{2}\right) .
$$

Combining these, we have

$$
\begin{aligned}
& x_{1} y_{2} \cdots y_{N} y_{1} y_{2} \cdots y_{N} M_{\infty}^{\prime}\left(R_{1}\right) \\
& \quad \subset y_{1} y_{2} \cdots y_{N} M_{\infty}^{\prime}\left(R_{2}\right)+y_{1} y_{2} \cdots y_{N} p M_{\infty}^{\prime}\left(R_{1}\right) \\
& \quad \subset M_{\infty}^{\prime}\left(p^{k} R_{1}\right)+p M_{\infty}^{\prime}\left(R_{2}\right)+y_{1} y_{2} \cdots y_{N} p M_{\infty}^{\prime}\left(R_{1}\right) .
\end{aligned}
$$

Simplifying and canceling $p$, we have

$$
y_{2}^{2} \cdots y_{N}^{2} M_{\infty}^{\prime}\left(R_{1}\right) \subset p^{k-1} M_{\infty}^{\prime}\left(R_{1}\right)+M_{\infty}^{\prime}\left(R_{2}\right)+y_{1} y_{2} \cdots y_{N} M_{\infty}^{\prime}\left(R_{1}\right) .
$$

Assume that $k>1$. Then projecting via $M_{\infty}^{\prime}\left(R_{1}\right) \rightarrow M_{\infty}^{\prime}\left(\bar{R}_{1}\right) \rightarrow M_{\infty}^{\prime}\left(\sigma_{1}\right)$, we have the inclusion

$$
y_{2}^{2} \cdots y_{N}^{2} M_{\infty}^{\prime}\left(\sigma_{1}\right) \subset y_{1} y_{2} \cdots y_{N} M_{\infty}^{\prime}\left(\sigma_{1}\right) .
$$

This is a contradiction since $M_{\infty}^{\prime}\left(\sigma_{1}\right)$ is free over (a power series ring over) $\mathbb{F} \llbracket y_{1}, \ldots, y_{N} \rrbracket$, being maximal Cohen-Macaulay over it. 
We deduce the following inequality.

COROllary 4.3.8. Assume that $R$ is 13-generic and let $\sigma_{1}, \sigma_{2} \in \mathrm{JH}(\bar{R})$. Then $\mathrm{d}_{\mathrm{gph}}\left(\sigma_{1}, \sigma_{2}\right) \geqslant \mathrm{d}_{\text {sat }}\left(\sigma_{1}, \sigma_{2}\right)$.

Proof. The proof proceeds by induction on $\mathrm{d}_{\mathrm{gph}}\left(\sigma_{1}, \sigma_{2}\right)$, the case $\mathrm{d}_{\mathrm{gph}}\left(\sigma_{1}, \sigma_{2}\right)=$ 1 being covered by Proposition 4.3.7. Pick a weight $\kappa \in \mathrm{JH}(\bar{R})$ distinct from $\sigma_{1}$ and $\sigma_{2}$ such that $\mathrm{d}_{\mathrm{gph}}\left(\sigma_{1}, \kappa\right)+\mathrm{d}_{\mathrm{gph}}\left(\kappa, \sigma_{2}\right)=\mathrm{d}_{\mathrm{gph}}\left(\sigma_{1}, \sigma_{2}\right)$. Then we have

$$
\begin{aligned}
\mathrm{d}_{\mathrm{gph}}\left(\sigma_{1}, \sigma_{2}\right) & =\mathrm{d}_{\mathrm{gph}}\left(\sigma_{1}, \kappa\right)+\mathrm{d}_{\mathrm{gph}}\left(\kappa, \sigma_{2}\right) \\
& \geqslant \mathrm{d}_{\mathrm{sat}}\left(\sigma_{1}, \kappa\right)+\mathrm{d}_{\mathrm{sat}}\left(\kappa, \sigma_{2}\right) \geqslant \mathrm{d}_{\mathrm{sat}}\left(\sigma_{1}, \sigma_{2}\right) .
\end{aligned}
$$

We conclude this section showing that, under appropriate genericity conditions on the Deligne-Lusztig representation $R$, the graph $\Gamma_{\text {rad }}\left(\bar{R}^{\sigma}\right)$ is predicted by the extension graph if its Loewy strata are predicted by the extension graph (which is a weaker assumption, a priori).

LemMA 4.3.9. Assume that $R$ is 13-generic. Let $\sigma_{1}, \sigma_{2} \in \mathrm{JH}(\bar{R})$ be such that $\mathrm{d}_{\mathrm{gph}}\left(\sigma_{1}, \sigma_{2}\right)=1$. Let us fix $\sigma \in \mathrm{JH}(\bar{R})$ as well as two saturated inclusions of lattices $R^{\sigma_{2}} \subseteq R^{\sigma}$ and $R^{\sigma_{1}} \subseteq R^{\sigma}$. Then either $R^{\sigma_{2}} \subseteq R^{\sigma_{1}}$ or $R^{\sigma_{1}} \subseteq R^{\sigma_{2}}$.

Proof. We have $\mathrm{d}_{\mathrm{gph}}\left(\sigma_{1}, \sigma_{2}\right)=\mathrm{d}_{\mathrm{sat}}\left(\sigma_{1}, \sigma_{2}\right)=1$ by Proposition 4.3.7. Hence, there exists an integer $k \in \mathbb{Z}$ such that

$$
p^{k+1} R^{\sigma_{1}} \subseteq R^{\sigma_{2}} \subseteq p^{k} R^{\sigma_{1}}
$$

is a chain of saturated inclusions of lattices (see Remark 4.3.5). The first inclusion implies that $p^{k+1} R^{\sigma_{1}} \subseteq R^{\sigma}$ so that $k+1 \geqslant 0$. The second inclusion implies that $p^{-k} R^{\sigma_{2}} \subset R^{\sigma}$ so that $-k \geqslant 0$. Hence, $k=0$ or -1 and the result follows.

Lemma 4.3.10. Assume that $R$ is 13-generic. Let $\sigma, \sigma_{1}, \sigma_{2} \in \mathrm{JH}(\bar{R})$ and fix saturated inclusions $R^{\sigma_{i}} \subseteq R^{\sigma}$ for $i=1$, 2. If $\mathrm{d}_{\mathrm{rad}}^{\sigma}\left(\sigma_{2}\right) \geqslant \mathrm{d}_{\mathrm{rad}}^{\sigma}\left(\sigma_{1}\right)$ and $\mathrm{d}_{\mathrm{gph}}\left(\sigma_{1}\right.$, $\left.\sigma_{2}\right)=1$, then $R^{\sigma_{2}} \subseteq R^{\sigma_{1}}$.

Proof. By Lemma 4.3.9, either $R^{\sigma_{1}} \subset R^{\sigma_{2}}$ or $R^{\sigma_{2}} \subset R^{\sigma_{1}}$. Suppose that the former holds. Then $Q_{\sigma}\left(\sigma_{1}\right) \subset Q_{\sigma}\left(\sigma_{2}\right)$, and thus $\mathrm{d}_{\mathrm{rad}}^{\sigma}\left(\sigma_{1}\right)>\mathrm{d}_{\mathrm{rad}}^{\sigma}\left(\sigma_{2}\right)$ by Lemma 4.3.3. This is a contradiction.

Definition 4.3.11. Assume that $R$ is 2-generic and let $\sigma \in \mathrm{JH}(\bar{R})$. We say that the radical strata of $\bar{R}^{\sigma}$ are predicted by the extension graph if $\mathrm{d}_{\mathrm{gph}}\left(\sigma, \sigma^{\prime}\right)=$ $\mathrm{d}_{\mathrm{rad}}^{\sigma}\left(\sigma^{\prime}\right)$ for all $\sigma^{\prime} \in \mathrm{JH}(\bar{R})$. 
Lemma 4.3.12. Assume that $R$ is 13-generic and let $\sigma \in \mathrm{JH}(\bar{R})$. If the radical strata of $\bar{R}^{\sigma}$ are predicted by the extension graph, then $\Gamma_{\mathrm{rad}}\left(\bar{R}^{\sigma}\right)$ is predicted by the extension graph with respect to $\sigma$.

Proof. Let $\sigma_{i}$ and $\sigma_{i+1}$ be elements of $\mathrm{JH}(\bar{R})$ such that $\mathrm{d}_{\mathrm{gph}}\left(\sigma, \sigma_{i}\right)=i$ and $\mathrm{d}_{\mathrm{gph}}(\sigma$, $\left.\sigma_{i+1}\right)=i+1$. As the radical strata of $\bar{R}^{\sigma}$ are predicted by the extension graph, we have $\mathrm{d}_{\mathrm{gph}}\left(\sigma, \sigma_{i}\right)=\mathrm{d}_{\mathrm{rad}}^{\sigma}\left(\sigma_{i}\right)$ and $\mathrm{d}_{\mathrm{gph}}\left(\sigma, \sigma_{i+1}\right)=\mathrm{d}_{\mathrm{rad}}^{\sigma}\left(\sigma_{i+1}\right)$.

If $\mathrm{d}_{\mathrm{gph}}\left(\sigma_{i}, \sigma_{i+1}\right)=1$, then $\sigma_{i+1} \in \mathrm{JH}\left(Q_{\sigma}\left(\sigma_{i}\right)\right)$ by Lemma 4.3.10. By Lemma 4.3.3, we have

$$
i+1=\mathrm{d}_{\mathrm{rad}}^{\sigma}\left(\sigma_{i+1}\right) \geqslant \mathrm{d}_{\mathrm{rad}}^{\sigma}\left(\sigma_{i}\right)+\mathrm{d}_{\mathrm{rad}}^{\sigma_{i}}\left(\sigma_{i+1}\right)=i+\mathrm{d}_{\mathrm{rad}}^{\sigma_{i}}\left(\sigma_{i+1}\right) .
$$

This implies that $\sigma_{i+1}$ appears in the second layer of the radical filtration of $Q_{\sigma}\left(\sigma_{i}\right)$, whose cosocle is isomorphic to $\sigma_{i}$ by Lemma 4.3.2(1). Then there is an edge from $\sigma_{i}$ to $\sigma_{i+1}$ in $\Gamma_{\text {rad }}\left(\bar{R}^{\sigma}\right)$.

Conversely, if there is an edge from $\sigma_{i}$ to $\sigma_{i+1}$ in $\Gamma_{\text {rad }}\left(\bar{R}^{\sigma}\right)$, then $\mathrm{d}_{\mathrm{gph}}\left(\sigma_{i}, \sigma_{i+1}\right)$ is 1 by Lemma 4.2.6.

4.3.2. Distance equalities. In this subsection, we define when a weight $\sigma \in$ $\mathrm{JH}(\bar{R})$ is maximally saturated. Crucially using Lemma 4.2.6, we show that if $\sigma$ is maximally saturated, then the graph, saturation, and cosocle distances from $\sigma$ are equal, and, therefore, the structure Theorem 4.1.9 holds for $\bar{R}^{\sigma}$. From now on, we assume that the Deligne-Lusztig representation $R$ is 13-generic.

From Proposition 2.3.5 and the definition of defect (Definition 2.8), it follows that

$$
\max \left\{\mathrm{d}_{\mathrm{gph}}(\sigma, \kappa), \kappa \in \mathrm{JH}(\bar{R})\right\}=3 f-\operatorname{Def}_{R}(\sigma) .
$$

Furthermore, if $\operatorname{Def}_{R}(\sigma)=0$, then there is a unique $\sigma^{\text {op }} \in \mathrm{JH}(\bar{R})$ which has maximal graph distance from $\sigma$.

Definition 4.3.13. Let $\sigma \in \operatorname{JH}(\bar{R})$. We say that the weight $\sigma$ is maximally saturated in $R$ if the following property holds:

If $\kappa \in \mathrm{JH}(\bar{R})$ verifies $\mathrm{d}_{\mathrm{gph}}(\sigma, \kappa)=3 f-\operatorname{Def}_{R}(\sigma)$, then $\mathrm{d}_{\mathrm{sat}}(\sigma, \kappa)=3 f-\operatorname{Def}_{R}(\sigma)$.

The following proposition motivates Definition 4.3.13.

Proposition 4.3.14. A weight $\sigma \in \mathrm{JH}(\bar{R})$ is maximally saturated if and only if $\mathrm{d}_{\mathrm{gph}}(\sigma, \kappa)=\mathrm{d}_{\mathrm{sat}}(\sigma, \kappa)$ for all $\kappa \in \mathrm{JH}(\bar{R})$. 
Proof. The 'if' part is clear. Let $\kappa \in \mathrm{JH}(\bar{R})$ be any weight and write $d \stackrel{\text { def }}{=} \mathrm{d}_{\mathrm{gph}}(\sigma$, $\kappa)$ and $D \stackrel{\text { def }}{=} 3 f-\operatorname{Def}_{R}(\sigma)$. There are weights $\sigma=\sigma_{0}, \sigma_{1}, \ldots, \sigma_{D}$ so that $\kappa \in\left\{\sigma_{0}, \sigma_{1}, \ldots, \sigma_{D}\right\} \subset \mathrm{JH}(\bar{R}), \mathrm{d}_{\mathrm{gph}}\left(\sigma_{i}, \sigma_{i+1}\right)=1$ for all $0 \leqslant i \leqslant D-1$ and $\mathrm{d}_{\mathrm{gph}}\left(\sigma, \sigma_{D}\right)=D$. It is then easy to see that $\mathrm{d}_{\mathrm{gph}}\left(\sigma_{i}, \sigma_{j}\right)=j-i$ if $0 \leqslant i \leqslant j \leqslant D$. We have the following chain of a priori inequalities

$$
\begin{aligned}
\mathrm{d}_{\text {sat }}\left(\sigma, \sigma_{D}\right) & \leqslant \mathrm{d}_{\text {sat }}(\sigma, \kappa)+\mathrm{d}_{\mathrm{sat}}\left(\kappa, \sigma_{D}\right) \\
& \leqslant \mathrm{d}_{\mathrm{gph}}(\sigma, \kappa)+\mathrm{d}_{\mathrm{gph}}\left(\kappa, \sigma_{D}\right) \\
& =\mathrm{d}_{\mathrm{gph}}\left(\sigma, \sigma_{D}\right) \\
& =\mathrm{d}_{\mathrm{sat}}\left(\sigma, \sigma_{D}\right),
\end{aligned}
$$

using Corollary 4.3.8 where the last equality holds by assumption. We conclude that the above inequalities are in fact equalities and that $\mathrm{d}_{\mathrm{sat}}(\sigma, \kappa)=\mathrm{d}_{\mathrm{gph}}(\sigma$, $\kappa)$.

The following lemma will be the key in relating the notions of saturation and cosocle distance.

LEMMA 4.3.15. Let $d<\ell \ell\left(\bar{R}^{\sigma}\right)$ and let

$$
\sigma_{d} \leftarrow \sigma_{d-1} \leftarrow \cdots \leftarrow \sigma_{1} \leftarrow \sigma_{0} \stackrel{\text { def }}{=} \sigma
$$

be an extension path in $\Gamma_{\mathrm{rad}}\left(\bar{R}^{\sigma}\right)$ (note that $\mathrm{d}_{\mathrm{rad}}^{\sigma}\left(\sigma_{i}\right)=i$ for all $i \in\{0, \ldots, d\}$ ). For each $i \in\{0, \ldots, d\}$, let us fix a saturated inclusion $R^{\sigma_{i}} \subseteq R^{\sigma}$. Then we have a chain of (saturated) inclusions

$$
R^{\sigma_{d}} \subseteq R^{\sigma_{d-1}} \subseteq \cdots \subseteq R^{\sigma_{1}} \subseteq R^{\sigma_{0}}
$$

Proof. Since $\mathrm{d}_{\mathrm{rad}}^{\sigma}\left(\sigma_{i+1}\right)=i+1 \geqslant i=\mathrm{d}_{\mathrm{rad}}^{\sigma}\left(\sigma_{i}\right)$ for $0 \leqslant i \leqslant d-1$, the result follows from Lemma 4.3.10.

We can use Lemma 4.3.15 to show that all three notions of distance from $\sigma$ agree in some particular situations.

Proposition 4.3.16. If $\sigma \in \mathrm{JH}(\bar{R})$ is maximally saturated, then $\Gamma_{\mathrm{rad}}\left(\bar{R}^{\sigma}\right)$ is predicted by the extension graph with respect to $\sigma$.

Proof. Assume that $\sigma$ is maximally saturated. By Lemma 4.3.12, it suffices to show that $\mathrm{d}_{\mathrm{gph}}\left(\sigma, \sigma^{\prime}\right)=\mathrm{d}_{\mathrm{rad}}^{\sigma}\left(\sigma^{\prime}\right)$ for all $\sigma^{\prime} \in \mathrm{JH}(\bar{R})$. Let $\sigma^{\prime} \in \mathrm{JH}(\bar{R})$ and $d=$ $\mathrm{d}_{\mathrm{gph}}\left(\sigma, \sigma^{\prime}\right)$. We have that $\operatorname{Hom}_{\mathrm{G}}\left(\sigma^{\prime}, \operatorname{gr}^{k}\left(\bar{R}^{\sigma}\right)\right) \neq 0$ for some $k \geqslant d$ by Corollary 
4.3.1. Assume for the sake of contradiction that $k>d$. By the definition of the radical filtration, we may, and do, fix an extension path of length $k+1$ in $\Gamma_{\text {rad }}\left(\bar{R}^{\sigma}\right)$ :

$$
\sigma^{\prime}=\sigma_{k} \leftarrow \sigma_{k-1} \leftarrow \cdots \leftarrow \sigma_{1} \leftarrow \sigma_{0}=\sigma .
$$

Since $\mathrm{d}_{\mathrm{gph}}\left(\sigma_{0}, \sigma_{k}\right)=d<k$ and $\mathrm{d}_{\mathrm{gph}}\left(\sigma_{0}, \sigma_{i}\right) \leqslant \mathrm{d}_{\mathrm{rad}}^{\sigma_{0}}\left(\sigma_{i}\right)=i$ for all $i$ by Corollary 4.3.1, there exists an index $i \in\{0, \ldots, k-1\}$ such that

(1) $\mathrm{d}_{\mathrm{gph}}\left(\sigma_{0}, \sigma_{i}\right)=i$ and

(2) $\mathrm{d}_{\mathrm{gph}}\left(\sigma_{0}, \sigma_{i+1}\right)<i+1$.

Moreover, since the extension graph is bipartite, we have that $\mathrm{d}_{\mathrm{gph}}\left(\sigma_{0}, \sigma_{i+1}\right) \leqslant$ $i-1$. By Lemma 4.3.15, there is a chain of saturated inclusions:

$$
R^{\sigma_{i+1}} \subseteq R^{\sigma_{i}} \subseteq \cdots \subseteq R^{\sigma_{1}} \subseteq R^{\sigma}
$$

where, in particular, $R^{\sigma_{i}} \subseteq R^{\sigma}$ is saturated as well. As $\mathrm{d}_{\mathrm{gph}}\left(\sigma_{0}, \sigma_{i+1}\right)=\mathrm{d}_{\mathrm{sat}}\left(\sigma_{0}\right.$, $\sigma_{i+1}$ ) by Proposition 4.3.14, we further have $p^{i-1} R^{\sigma} \subseteq R^{\sigma_{i+1}}$. We conclude that $p^{i-1} R^{\sigma} \subseteq R^{\sigma_{i}} \subseteq R^{\sigma}$ and, hence, that $\mathrm{d}_{\mathrm{sat}}\left(\sigma_{0}, \sigma_{i}\right) \leqslant \bar{i}-1$. This contradicts (1) since $\mathrm{d}_{\mathrm{gph}}\left(\sigma_{0}, \sigma_{i}\right)=\mathrm{d}_{\mathrm{sat}}\left(\sigma_{0}, \sigma_{i}\right)$ by Proposition 4.3.14.

We now use Theorem 4.2.16 to prove that lower alcove weights of defect zero are maximally saturated.

Proposition 4.3.17. Let $R$ be a 13-generic Deligne-Lusztig representation and let $\sigma \in \mathrm{JH}(\bar{R})$ be a constituent with $\operatorname{Def}_{R}(\sigma)=0$. Assume that $\sigma \cong F(\lambda)$ where $\lambda \in X_{1}(\underline{T})$ is in alcove $\underline{A}$. Then $\sigma$ is maximally saturated in $\bar{R}$.

Proof. By Theorem 4.2.16, we can and do fix an extension path in $\Gamma_{\text {rad }}\left(\bar{R}^{\sigma}\right)$ with starting point $\sigma_{0} \stackrel{\text { def }}{=} \sigma: \sigma^{\mathrm{op}}=\sigma_{3 f} \leftarrow \sigma_{3 f-1} \leftarrow \cdots \leftarrow \sigma_{1} \leftarrow \sigma_{0}$. By Lemma 4.3.15, we have a sequence of saturated inclusions:

$$
R^{\sigma} \supseteq R^{\sigma_{1}} \supseteq \cdots \supseteq R^{\sigma_{3 f-1}} \supseteq R^{\sigma^{\mathrm{op}}}
$$

where $R^{\sigma^{\text {op }}} \subseteq R^{\sigma}$ is itself saturated. For each $0 \leqslant i \leqslant 3 f-1$, let $n_{i}$ be $\mathrm{d}_{\text {sat }}\left(\sigma_{i}\right.$, $\left.\sigma^{\text {op }}\right)$. It suffices to show that $n_{0}=\mathrm{d}_{\text {gph }}\left(\sigma_{0}, \sigma^{\text {op }}\right)=3 f$.

By Theorem 4.2.16, the reduction of the lattice in the dual Deligne-Lusztig representation $R^{*}$ with cosocle $\sigma^{\vee}$ is rigid and $\Gamma\left({\overline{\left(R^{*}\right)}}^{\sigma^{\vee}}\right)$ is predicted by the extension graph with respect to $\sigma^{\vee}$. Noting that the reduction of the dual of a lattice is the dual of the reduction of a lattice and using Proposition 4.1.7, we see that $\Gamma_{\text {rad }}\left(\bar{R}^{\sigma^{\text {op }}}\right)$ is predicted by the extension graph with respect to $\sigma^{\text {op }}$. 
In particular, $\sigma_{0} \leftarrow \sigma_{1} \leftarrow \cdots \leftarrow \sigma_{3 f-1} \leftarrow \sigma_{3 f}=\sigma^{\text {op }}$ is an extension path in $\Gamma_{\text {rad }}\left(\bar{R}^{\sigma}\right)$, and, hence, from Lemma 4.3.15, we deduce a chain of (saturated) inclusions

$$
R^{\sigma^{\text {op }}} \supseteq p^{n_{3 f-1}} R^{\sigma_{3 f-1}} \supseteq \cdots \supseteq p^{n_{1}} R^{\sigma_{1}} \supseteq p^{n_{0}} R^{\sigma}
$$

where we necessarily have $n_{i-1}>n_{i}$ for all $i$ (as $R^{\sigma_{i+1}} \subseteq R^{\sigma_{i}}$ is saturated). In particular, $n_{0} \geqslant 3 f$. On the other hand, Corollary 4.3 .8 implies that $n_{0} \leqslant 3 f$ so that $n_{0}=3 f$.

4.3.3. Induction on defect. In this subsection, we show inductively that all weights are maximally saturated, starting from lower alcove weights as in Proposition 4.3.17. We conclude the section with the proof of Theorem 4.1.9. We first start with the defect zero case.

Lemma 4.3.18. Let $R$ be a 13-generic Deligne-Lusztig representation. If $\sigma \in$ $\mathrm{JH}(\bar{R})$ and $\operatorname{Def}_{R}(\sigma)=0$, then $\sigma$ is maximally saturated.

Proof. We claim that if $\sigma \stackrel{\text { def }}{=} \sigma_{0}, \sigma_{1} \in \mathrm{JH}(\bar{R})$ such that $\operatorname{Def}_{R}\left(\sigma_{0}\right)=0, \operatorname{Def}_{R}\left(\sigma_{1}\right)=$ $0, \mathrm{~d}_{\mathrm{gph}}\left(\sigma_{0}, \sigma_{1}\right)=1$, and $\sigma_{0}$ is maximally saturated, then $\sigma_{1}$ is maximally saturated. The result then follows from Proposition 4.3.17 and an easy induction argument.

By Proposition 4.3.16, the graph $\Gamma_{\text {rad }}\left(\bar{R}^{\sigma}\right)$ is predicted by the extension graph. By duality (cf. the proof of Proposition 4.3.17), the graph $\Gamma_{\text {rad }}\left(\bar{R}^{\sigma^{\text {op }}}\right)$ is also predicted by the extension graph. Hence, we may and do choose two extension paths, of starting point $\sigma$, in the graph $\Gamma_{\text {rad }}\left(\bar{R}^{\sigma_{0}}\right)$ having the form: $\sigma_{0}^{\text {op }} \leftarrow$ $\sigma_{3 f-1}^{\prime} \leftarrow \cdots \leftarrow \sigma_{2}^{\prime} \leftarrow \sigma_{1} \leftarrow \sigma_{0}$ and $\sigma_{0}^{\mathrm{op}} \leftarrow \sigma_{1}^{\mathrm{op}} \leftarrow \sigma_{3 f-2}^{\prime \prime} \leftarrow \cdots \leftarrow \sigma_{1}^{\prime \prime} \leftarrow \sigma_{0}$ where $\sigma_{i}^{\prime}, \sigma_{i}^{\prime \prime} \in \mathrm{JH}\left(\bar{R}^{\sigma}\right)$. As the graph $\Gamma_{\text {rad }}\left(\bar{R}^{\sigma^{\text {op }}}\right)$ is predicted by the extension graph, the extension paths above induce extensions paths in $\bar{R}^{\sigma^{\text {op }}}$ by 'reversing the arrows and the endpoints'. Let us fix saturated inclusions of lattices $R^{\sigma_{i}^{\prime}}$, $R^{\sigma_{i}^{\prime \prime}} \subseteq R^{\sigma}, R^{\sigma_{1}}, R^{\sigma_{1}^{\mathrm{op}}} \subseteq R^{\sigma^{\mathrm{op}}}$. Since $\sigma$ and $\sigma^{\text {op }}$ are maximally saturated, we deduce that $p^{3 f-i} R^{\sigma_{i}^{\prime}}, \overline{p^{3 f-i}} R^{\sigma_{i}^{\prime \prime}} \subseteq R^{\sigma^{\text {op }}}, p^{3 f-1} R^{\sigma_{1}}, p R^{\sigma_{1}^{\text {op }}} \subseteq R^{\sigma^{\text {op }}}$ are saturated inclusions as well.

By Lemma 4.3.15, we deduce the following chain of saturated inclusions:

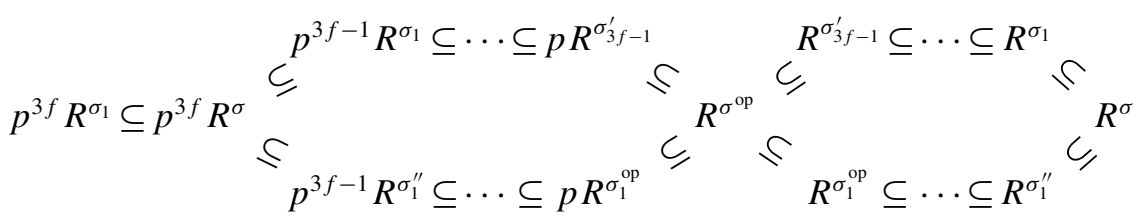


We claim that the inclusions $p^{3 f} R^{\sigma_{1}} \subseteq p R^{\sigma_{1}^{\text {op }}}$ and $p R^{\sigma_{1}^{\text {op }}} \subseteq R^{\sigma_{1}}$, obtained by composing the saturated inclusions above, are saturated.

We first show that $p^{3 f} R^{\sigma_{1}} \subseteq p R^{\sigma_{1}^{\text {op }}}$ is saturated. If not, then we obtain a chain of saturated inclusion $p^{3 f-1} R^{\sigma_{1}} \subseteq p R^{\sigma_{1}^{\mathrm{op}}} \subseteq R^{\sigma^{\mathrm{op}}}$. We deduce that $\sigma_{1}$ is a constituent in image $Q_{\sigma^{\text {op }}}\left(\sigma_{1}^{\mathrm{op}}\right)$ and hence $\mathrm{d}_{\mathrm{rad}}^{\sigma^{\mathrm{op}}}\left(\sigma_{1}\right) \geqslant \mathrm{d}_{\mathrm{rad}}^{\sigma^{\mathrm{op}}}\left(\sigma_{1}^{\mathrm{op}}\right)+\mathrm{d}_{\mathrm{rad}}^{\sigma_{1}^{\mathrm{op}}}\left(\sigma_{1}\right) \geqslant$ $\mathrm{d}_{\mathrm{gph}}\left(\sigma^{\mathrm{op}}, \sigma_{1}^{\mathrm{op}}\right)+\mathrm{d}_{\mathrm{gph}}\left(\sigma_{1}^{\mathrm{op}}, \sigma_{1}\right) \geqslant 3 f+1$ by Corollary 4.3.1 and Lemma 4.3.3. On the other hand, as the graph $\Gamma_{\text {rad }}\left(\bar{R}^{\sigma_{0}^{\text {op }}}\right)$ is predicted by the extension graph, we have that $\mathrm{d}_{\text {rad }}^{\sigma^{\text {op }}}\left(\sigma_{1}\right)=\mathrm{d}_{\mathrm{gph}}\left(\sigma^{\mathrm{op}}, \sigma_{1}\right)=3 f-1$ contradiction.

The evident analogue of the previous argument shows that $p R^{\sigma_{1}^{\text {op }}} \subseteq R^{\sigma_{1}}$ is saturated as well. Hence, $\mathrm{d}_{\text {sat }}\left(\sigma_{1}, \sigma_{1}^{\mathrm{op}}\right)=3 f$.

We now give the induction argument.

Proposition 4.3.19. Let $R$ be a 13-generic Deligne-Lusztig representation. Then any constituent $\sigma \in \mathrm{JH}(\bar{R})$ is maximally saturated in $\bar{R}$.

Proof. We induct on the defect $\delta \stackrel{\text { def }}{=} \operatorname{Def}_{R}(\sigma)$ for $\sigma \in \mathrm{JH}(\bar{R})$. The case $\delta=0$ holds by Lemma 4.3.18. Suppose that $\delta>0$. To ease notation, let $d \stackrel{\text { def }}{=} 3 f-\delta$ and pick a weight $\sigma_{d} \in \mathrm{JH}(\bar{R})$ such that $\mathrm{d}_{\mathrm{gph}}\left(\sigma, \sigma_{d}\right)=d$ (in other words, $\sigma_{d} \in$ $\mathrm{JH}(\bar{R})$ is at maximal graph distance from $\sigma)$; we will show that $d=\mathrm{d}_{\text {sat }}\left(\sigma, \sigma_{d}\right)$. Note that $\operatorname{Def}_{R}\left(\sigma_{d}\right) \leqslant \operatorname{Def}_{R}(\sigma)$. If $\operatorname{Def}_{R}\left(\sigma_{d}\right)<\operatorname{Def}_{R}(\sigma)$, then $\sigma_{d}$ is maximally saturated (by induction on $\operatorname{Def}_{R}\left(\sigma_{d}\right)$ ), and, hence, $\mathrm{d}_{\mathrm{gph}}\left(\sigma, \sigma_{d}\right)=\mathrm{d}_{\text {sat }}\left(\sigma, \sigma_{d}\right)$ by Proposition 4.3.14.

We now consider the case $\operatorname{Def}_{R}\left(\sigma_{d}\right)=\operatorname{Def}_{R}(\sigma)$. By a direct check on the extension graph, we see that there exists $\sigma_{d-1} \in \mathrm{JH}(\bar{R})$ with $\mathrm{d}_{\text {gph }}\left(\sigma_{d}, \sigma_{d-1}\right)=1$ and $\operatorname{Def}_{R}\left(\sigma_{d-1}\right)=\operatorname{Def}_{R}(\sigma)-1$. Note that $\mathrm{d}_{\mathrm{gph}}\left(\sigma_{d-1}, \sigma\right)=d-1$. By induction, the weight $\sigma_{d-1}$ is maximally saturated; hence, $\mathrm{d}_{\text {sat }}\left(\sigma_{d-1}, \kappa\right)=\mathrm{d}_{\mathrm{gph}}\left(\sigma_{d-1}, \kappa\right)=$ $\mathrm{d}_{\mathrm{rad}}^{\sigma_{d-1}}(\kappa)$ for all $\kappa \in \mathrm{JH}(\bar{R})$ by Propositions 4.3.14 and 4.3.16.

Let $R^{\sigma_{d-1}} \subseteq R^{\sigma}, R^{\sigma_{d}} \subseteq R^{\sigma}$ be saturated inclusions of lattices. By Lemma 4.3.9, we are in one of the following situations:

(1) $R^{\sigma_{d}} \subseteq R^{\sigma_{d-1}} \subseteq R^{\sigma}$;

(2) $R^{\sigma_{d-1}} \subseteq R^{\sigma_{d}} \subseteq R^{\sigma}$,

where the inclusions are all saturated.

Case (1). Taking $\kappa=\sigma$ in the above, we have that $\mathrm{d}_{\mathrm{sat}}\left(\sigma, \sigma_{d-1}\right)=d-1$ and, hence, obtain chains of saturated inclusions:

$$
\begin{array}{cc}
p^{d-1} R^{\sigma} \subseteq & R^{\sigma_{d-1}} \subseteq R^{\sigma} \\
& \text { UI } \\
& R^{\sigma_{d}}
\end{array}
$$


Assume for the sake of contradiction that $\mathrm{d}_{\text {sat }}\left(\sigma, \sigma_{d}\right)<d$, that is, that we have a factorization

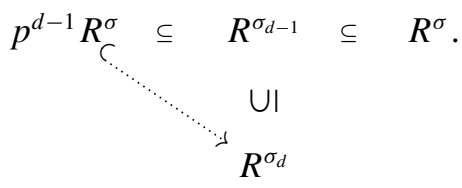

Then, we necessarily have that $\mathrm{d}_{\mathrm{sat}}\left(\sigma, \sigma_{d}\right)=d-1$. We obtain a commutative diagram:

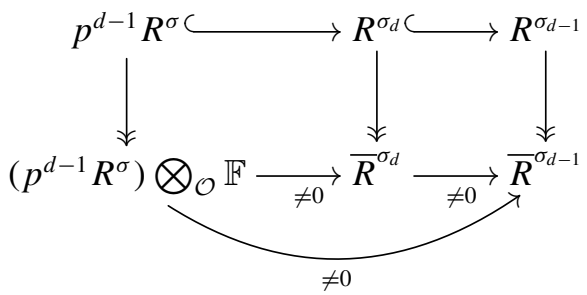

where the lower arrows are all nonzero.

In particular, $\sigma$ is a constituent of $Q_{\sigma_{d-1}}\left(\sigma_{d}\right)$. By Corollary 4.3.1 and Lemma 4.3.3, we have $\mathrm{d}_{\mathrm{rad}}^{\sigma_{d-1}}(\sigma) \geqslant \mathrm{d}_{\mathrm{rad}}^{\sigma_{d-1}}\left(\sigma_{d}\right)+\mathrm{d}_{\mathrm{rad}}^{\sigma_{d}}(\sigma) \geqslant \mathrm{d}_{\mathrm{gph}}\left(\sigma_{d-1}, \sigma_{d}\right)+\mathrm{d}_{\mathrm{gph}}\left(\sigma_{d}, \sigma\right)=$ $d+1$. On the other hand, as $\sigma_{d-1}$ is maximally saturated, $\mathrm{d}_{\mathrm{rad}}^{\sigma_{d-1}}(\sigma)=d-1$, a contradiction.

Case (2). We now have a commutative diagram

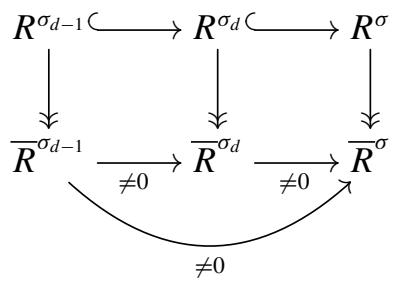

where again the lower arrows are nonzero. Exactly as in the previous case, we deduce that $\mathrm{d}_{\mathrm{rad}}^{\sigma}\left(\sigma_{d-1}\right) \geqslant \mathrm{d}_{\mathrm{rad}}^{\sigma}\left(\sigma_{d}\right)+1 \geqslant d+1$.

We therefore may, and do, fix an extension path in the radical filtration of $\bar{R}^{\sigma}$ :

$$
\sigma_{d-1}=\sigma_{k}^{\prime} \leftarrow \sigma_{k-1}^{\prime} \leftarrow \cdots \leftarrow \sigma_{1}^{\prime} \leftarrow \sigma_{0}^{\prime}=\sigma
$$

For notational convenience, we set $\kappa_{i} \stackrel{\text { def }}{=} \sigma_{k-i}^{\prime}$. As $k \geqslant d+1$, and as $\sigma_{d-1}$ is maximally saturated, we deduce as in the proof of Proposition 4.3.16, the existence of an index $i \in\{0, \ldots, k-1\}$ such that

(1) $\mathrm{d}_{\mathrm{gph}}\left(\kappa_{0}, \kappa_{i}\right)=\mathrm{d}_{\mathrm{sat}}\left(\kappa_{0}, \kappa_{i}\right)=i$ and 
(2) $\mathrm{d}_{\mathrm{gph}}\left(\kappa_{0}, \kappa_{i+1}\right)=\mathrm{d}_{\mathrm{sat}}\left(\kappa_{0}, \kappa_{i+1}\right)<i+1$, and actually $\mathrm{d}_{\mathrm{gph}}\left(\kappa_{0}, \kappa_{i+1}\right) \leqslant i-1$.

Fix a chain of saturated inclusions $R^{\kappa_{0}} \subseteq R^{\kappa_{1}} \subseteq \cdots \subseteq R^{\kappa_{k}}$. Item (2) implies that $R^{\kappa_{0}} \subseteq R^{\kappa_{i+1}} \subseteq p^{-i+1} R^{\kappa_{0}}$. The induced inclusion $R^{\kappa_{i}} \subseteq R^{\kappa_{i+1}} \subseteq p^{-i+1} R^{\kappa_{0}}$ contradicts item (1).

Proof of Theorem 4.1.9. (5) follows from Propositions 4.3.14 and 4.3.19. Items (1) and (2) follow from Propositions 4.3.16 and 4.3.19. Note that $\Gamma_{\text {rad }}\left(\bar{R}^{\sigma}\right)$ is a subgraph of $\Gamma\left(\bar{R}^{\sigma}\right)$. Using Lemma 4.2.6 and the fact that $\Gamma\left(\bar{R}^{\sigma}\right)$ is acyclic, $\Gamma\left(\bar{R}^{\sigma}\right)$ must in fact be $\Gamma_{\text {rad }}\left(\bar{R}^{\sigma}\right)$. This implies (3).

\section{Breuil's Conjectures}

In this section, we deduce generalizations of Breuil's conjectures on mod $p$ multiplicity one and lattices from the results in Sections 3-4. In Sections 5.15.2, we prove a version of Breuil's conjectures for abstract patching functors. Finally, we deduce the main results in Section 5.3.

5.1. Cyclicity for patching functors. In this subsection, we show that certain patched modules for tame types are locally free of rank one over the corresponding local deformation space using several inductive steps. The base case is Lemma 5.1.3. Each inductive step uses one of the two arguments in [EGS15, Section 10]. It is here that the results of Section 3.6.3 enter.

Recall from Section 1.4 that $\mathcal{S}$ is a finite set so that for each $\widetilde{v} \in \mathcal{S}, F_{\widetilde{v}}$ is a finite unramified extension of $\mathbb{Q}_{p}$ of degree $f_{\widetilde{v}}$. For each $\widetilde{v}$, let $\bar{\rho}_{\widetilde{v}}: G_{F_{\tilde{v}}} \rightarrow \mathrm{GL}_{3}(\mathbb{F})$ be a 10-generic semisimple continuous Galois representation and let $\bar{\rho}_{\mathcal{S}}$ be $\left(\bar{\rho}_{\widetilde{v}}\right)_{\tilde{v} \in \mathcal{S}}$. Recall the weak minimal patching functor setting of Section 3.5. Suppose that $\left.\bar{\rho}_{\mathcal{S}}\right|_{I_{\mathcal{S}}} \cong \bar{\tau}_{\mathcal{S}}(s, \mu)$ for $s \in \underline{W}$ and $\mu \in X^{*}(\underline{T})$.

Let $K$ be $\prod_{\tilde{v} \in \mathcal{S}} K_{\widetilde{v}}$. Suppose that $M_{\infty}$ is a weak minimal patching functor for $\bar{\rho}_{\mathcal{S}}$ (cf. Definition 3.5.1). For each $\widetilde{v} \in \mathcal{S}$, let $\tau_{\widetilde{v}}$ be a 13-generic tame inertial type for $F_{\widetilde{v}}$. Recall $R_{\mathfrak{M}_{\tilde{v}}, \bar{\rho}_{\widetilde{v}}}^{\tau_{\widetilde{v}}, \square}$ from [LLHLM18, Definition 5.10]. We let $\tau_{\mathcal{S}}$ be $\left(\tau_{\widetilde{v}}\right)_{\widetilde{v}}$ so that $\sigma\left(\tau_{\mathcal{S}}\right)$ is the $K$-module $\bigotimes_{\widetilde{v} \in \mathcal{S}} \sigma\left(\tau_{\widetilde{v}}\right)$. Let

$$
R_{\infty}^{\prime}\left(\tau_{\mathcal{S}}\right) \stackrel{\text { def }}{=} R_{\infty}\left(\tau_{\mathcal{S}}\right) \widehat{\otimes}_{\left(\widehat{\otimes}_{\tilde{v} \in \mathcal{S}} R_{\tilde{v}}^{\tau \widetilde{v}}\right)}\left(\widehat{\bigotimes}_{\widetilde{v} \in \mathcal{S}} R_{\mathfrak{M}_{\widetilde{v}}, \bar{\rho}_{\widetilde{v}}, \square}^{\bar{\rho}_{\widetilde{v}}}\right)
$$

and

$$
\left.M_{\infty}^{\prime}(V) \stackrel{\text { def }}{=} M_{\infty}(V) \widehat{\otimes}_{(\widehat{\otimes} \widetilde{v} \in \mathcal{S}} R_{\tilde{v}}^{\tau}\right)\left(\widehat{\bigotimes}_{\widetilde{v} \in \mathcal{S}} R_{\mathfrak{M}_{\tilde{v}}, \bar{\rho}_{\widetilde{v}}, \bar{\beta}_{\tilde{v}}, \square}^{\tau_{v}}\right)
$$

for any subquotient $V$ of a lattice in $\sigma\left(\tau_{\mathcal{S}}\right)$. 
We assume that $R_{\infty}\left(\tau_{\mathcal{S}}\right)$ is nonzero. Then there exists $\widetilde{w}=\left(\widetilde{w}_{\widetilde{v}}\right)_{\tilde{v} \in \mathcal{S}}=$ $\left(\widetilde{w}_{i}\right)_{i \in \mathcal{J}} \in \operatorname{Adm}^{\vee}(\eta)$ such that $\tau_{\mathcal{S}} \cong \tau_{\mathcal{S}}\left(s\left(w^{*}\right)^{-1}, \mu+s\left(\widetilde{w}^{*}\right)^{-1}(0)\right)$ with the lowest alcove presentation $\left(s\left(w^{*}\right)^{-1}, \mu+s\left(\widetilde{w}^{*}\right)^{-1}(0)-\eta\right)$ by Theorem 3.3.12 and Proposition 3.4.1. Note that $\widetilde{w}_{\widetilde{v}}=\widetilde{w}\left(\bar{\rho}_{\widetilde{v}}, \tau_{\widetilde{v}}\right)$. Then, as explained in Section 3.6.1, $\operatorname{Spf} \bar{R}_{\infty}^{\prime}\left(\tau_{\mathcal{S}}\right)$ (respectively $\operatorname{Spf} R_{\infty}^{\prime}\left(\tau_{\mathcal{S}}\right)$ ) is formally smooth over $\operatorname{Spf}\left(\widehat{\bigotimes}_{i \in \mathcal{J}} \bar{R}_{\mathfrak{M}_{\tilde{v}}, \widetilde{w}_{i}}^{\text {expl, }}\right)$ (respectively $\operatorname{Spf}\left(\widehat{\bigotimes}_{i \in \mathcal{J}} R_{\overline{\mathfrak{M}}_{\tilde{v}}, \widetilde{w}_{i}}^{\text {expl, }}\right)$ if $\ell\left(\widetilde{w}_{i}\right)>1$ for all $i \in$ $\mathcal{J})$, where $i=\left(\widetilde{v}, i_{\widetilde{v}}\right)$. We consider $\bar{R}_{\infty}^{\prime}\left(\tau_{\mathcal{S}}\right)$ (respectively $R_{\infty}^{\prime}\left(\tau_{\mathcal{S}}\right)$ ) both as a $\widehat{\bigotimes}_{i \in \mathcal{J}} \bar{R}_{\bar{M}_{\tilde{v}}, \widetilde{w}_{i}}^{\text {expl, }}$-algebra and a $\widehat{\bigotimes}_{\widetilde{v} \in \mathcal{S}} \bar{R}_{\bar{\rho}_{\widetilde{v}}}^{\square}$-algebra (respectively a $\widehat{\bigotimes}_{i \in \mathcal{J}} R_{\overline{\mathfrak{M}}_{\tilde{v}}, \widetilde{w}_{i}}^{\text {exl, }}$. algebra and a $\widehat{\bigotimes}_{\widetilde{v} \in \mathcal{S}} R_{\bar{\rho}_{\tilde{v}}}^{\square}$-algebra). It is easy to see that $M_{\infty}^{\prime}(V)$ is a cyclic $R_{\infty}^{\prime}\left(\tau_{\mathcal{S}}\right)$ module if and only if $M_{\infty}(V)$ is a cyclic $R_{\infty}\left(\tau_{\mathcal{S}}\right)$-module. Moreover, $M_{\infty}^{\prime}(V)$ is always maximal Cohen-Macaulay over its support. It will often be more convenient to prove that $M_{\infty}^{\prime}(V)$ is a cyclic $R_{\infty}^{\prime}\left(\tau_{\mathcal{S}}\right)$-module, and we will switch to $M_{\infty}^{\prime}(V)$ without comment. If $\sigma \in \mathrm{JH}\left(\overline{\sigma\left(\tau_{\mathcal{S}}\right)}\right)$, then recall from Section 4.1.2 that $\sigma\left(\tau_{\mathcal{S}}\right)^{\sigma}$ is the unique $\mathcal{O}$-lattice up to homothety in $\sigma\left(\tau_{\mathcal{S}}\right)$ with cosocle $\sigma$.

THEOREM 5.1.1. Let $\tau_{\mathcal{S}}$ be a 13-generic tame type and $\sigma \stackrel{\text { def }}{=} F(\lambda) \in W^{?}\left(\bar{\rho}_{\mathcal{S}}, \tau_{\mathcal{S}}\right)$ such that for all $i \in \mathcal{J}$,

$$
\lambda_{\pi^{-1}(i)} \in X_{1}(T) \text { is in alcove } C_{1} \text { if } \ell\left(\widetilde{w}_{i}^{*}\right) \leqslant 1 .
$$

Then $M_{\infty}\left(\sigma\left(\tau_{\mathcal{S}}\right)^{\sigma}\right)$ is free of rank one as a $R_{\infty}\left(\tau_{\mathcal{S}}\right)$-module.

The proof of Theorem 5.1.1 proceeds by proving cases of increasing complexity. We distinguish five steps in the argument.

LEMMA 5.1.2. If $\sigma \in W^{?}\left(\bar{\rho}_{\mathcal{S}}\right)$, then $M_{\infty}(\sigma)$ is a cyclic $R_{\infty}\left(\tau_{\mathcal{S}}\right)$-module.

Proof. The proof follows the methods of Diamond and Fujiwara [Dia97]. Note that the support of $M_{\infty}(\sigma)$ is formally smooth as can be checked from Theorem 3.5.2. Since $M_{\infty}(\sigma)$ is maximal Cohen-Macaulay over its formally smooth support by Definition 3.5.1(3), it is free over its support by the Auslander-Buchsbaum-Serre theorem and the Auslander-Buchsbaum formula. Since $e\left(M_{\infty}(\sigma)\right)=1$ by Theorem 3.5.2, the free rank is one.

If $V$ is a $\varpi$-torsion $K$-module satisfying the hypotheses of Lemma 3.6.2, we write $R_{\infty}(V)$ to denote the quotient $R_{\infty} / I(V)$. For the rest of this subsection, we assume that the weight $\sigma$ satisfies the conditions of Theorem 5.1.1.

Lemma 5.1.3. Assume that $\ell\left(\widetilde{w}_{i}^{*}\right)>1$ for all $i \in \mathcal{J}$. Let $\sigma \in W^{?}\left(\bar{\rho}_{\mathcal{S}}, \tau_{\mathcal{S}}\right)$ and let $V$ be a nonzero quotient of $\bar{\sigma}\left(\tau_{\mathcal{S}}\right)^{\sigma}$. Then $M_{\infty}(V)$ is a cyclic $R_{\infty}\left(\tau_{\mathcal{S}}\right)$-module. 
Proof. If $R_{\infty}^{\prime}\left(\tau_{\mathcal{S}}\right)$ is formally smooth over $\mathcal{O}$, then the result follows from Lemma 5.1.2. Suppose otherwise. We use the notation of the proof of Lemma 4.3.7 with $R=\sigma\left(\tau_{\mathcal{S}}\right)$. Recall that for $\sigma \in \mathrm{JH}\left(\overline{\sigma\left(\tau_{\mathcal{S}}\right)}\right), \mathfrak{p}_{S}(\sigma)=\left(z_{j}(\sigma)\right)_{j=1}^{N}+(p)$. By Lemma 3.6.10, for $\sigma_{1}$ and $\sigma_{2} \in W^{?}\left(\bar{\rho}_{\mathcal{S}}, \tau_{\mathcal{S}}\right)$, we have

$$
\#\left(\left\{z_{j}\left(\sigma_{1}\right)\right\}_{j} \Delta\left\{z_{j}\left(\sigma_{2}\right)\right\}_{j}\right)=2 \mathrm{~d}_{\mathrm{gph}}\left(\sigma_{1}, \sigma_{2}\right) .
$$

Let $\sigma_{1} \in W^{?}\left(\bar{\rho}_{\mathcal{S}}, \tau_{\mathcal{S}}\right)$ be such that $\mathrm{d}_{\mathrm{gph}}\left(\sigma, \sigma_{1}\right)=1$. Furthermore, fix a saturated inclusion $\sigma\left(\tau_{\mathcal{S}}\right)^{\sigma_{1}} \hookrightarrow \sigma\left(\tau_{\mathcal{S}}\right)^{\sigma}$. Letting $M \stackrel{\text { def }}{=} M_{\infty}^{\prime}\left(\sigma\left(\tau_{\mathcal{S}}\right)^{\sigma}\right)$ and $M_{1} \stackrel{\text { def }}{=}$ $M_{\infty}^{\prime}\left(\sigma\left(\tau_{\mathcal{S}}\right)^{\sigma_{1}}\right)$, we have a map $M_{1} \rightarrow M$. By Proposition 4.3.7, $\sigma\left(\tau_{\mathcal{S}}\right)^{\sigma} / \sigma\left(\tau_{\mathcal{S}}\right)^{\sigma_{1}}$ is $p$-torsion, with Jordan-Hölder factors determined by Theorem 4.1.9. By the proofs of Lemmas 3.6.2 and 4.3.7, the scheme-theoretic support of $M / M_{1}$ in Spec $S$ is

$$
\operatorname{Spec}\left(S / \bigcap_{\sigma^{\prime} \in \operatorname{JH}\left(\sigma\left(\tau_{\mathcal{S}}\right)^{\sigma} / \sigma\left(\tau_{\mathcal{S}}\right)^{\left.\sigma_{1}\right)}\right.} \mathfrak{p}_{S}\left(\sigma^{\prime}\right)\right) \text {. }
$$

The sets $\left\{z_{j}\left(\sigma_{1}\right)\right\}_{j}$ and $\left\{z_{j}(\sigma)\right\}_{j}$ differ at exactly one component, say $j_{1}$. The equation (5.2) determines $\mathfrak{p}_{S}\left(\sigma^{\prime}\right)$ for all $\sigma^{\prime} \in \mathrm{JH}\left(\overline{\sigma\left(\tau_{\mathcal{S}}\right)}\right)$ from which one checks that $z_{j_{1}}(\sigma)$ is in $\mathfrak{p}_{S}\left(\sigma^{\prime}\right)$ for any $\sigma^{\prime} \in \operatorname{JH}\left(\sigma\left(\tau_{\mathcal{S}}\right)^{\sigma} / \sigma\left(\tau_{\mathcal{S}}\right)^{\sigma_{1}}\right)$. By (5.3), we see that $\operatorname{coker}\left(M_{1} \rightarrow M\right)$ is killed by $z_{j_{1}}(\sigma)$. Similarly, we have that $\operatorname{coker}(p M \rightarrow$ $\left.M_{1}\right)$ is annihilated by $z_{j_{1}}\left(\sigma_{1}\right)$. Hence, we have inclusions $z_{j_{1}}(\sigma) M \subset M_{1}$ and $z_{j_{1}}\left(\sigma_{1}\right) M_{1} \subset p M$. Combining these, we have that $p M=z_{j_{1}}(\sigma) z_{j_{1}}\left(\sigma_{1}\right) M \subset$ $z_{j_{1}}\left(\sigma_{1}\right) M_{1} \subset p M$. We conclude that the above inclusions are equalities.

Applying the above argument for all $\sigma_{1} \in W^{?}\left(\bar{\rho}_{\mathcal{S}}, \tau_{\mathcal{S}}\right)$ at distance one from $\sigma$, we have that $M_{\infty}^{\prime}(\sigma) \cong M_{\infty}^{\prime}\left(\sigma\left(\tau_{\mathcal{S}}\right)^{\sigma}\right) /\left(\varpi,\left\{z_{i}(\sigma)\right\}_{i}\right)$. The left-hand side, and hence the right-hand side, is cyclic by Lemma 5.1.2. We conclude that $M_{\infty}^{\prime}\left(\bar{\sigma}\left(\tau_{\mathcal{S}}\right)^{\sigma}\right)$ is cyclic by Nakayama's lemma. Since $M_{\infty}^{\prime}(V)$ is a nonzero quotient of $M_{\infty}^{\prime}\left(\bar{\sigma}\left(\tau_{\mathcal{S}}\right)^{\sigma}\right)$, it too is cyclic.

As in Section 2.3, let $\sigma_{(\omega, a)} \stackrel{\text { def }}{=} F\left(\mathfrak{T r}_{\mu}(s \omega, a)\right.$ ) (where $\left.(s \omega, a) \in \underline{\Lambda}_{W}^{\mu} \times \mathcal{A}\right)$. Then $W^{?}\left(\bar{\rho}_{\mathcal{S}}\right)=\left\{\sigma_{(\omega, a)}:(\omega, a) \in r(\Sigma)\right\}$. Similarly, for $\widetilde{w} \in \operatorname{Adm}^{\vee}(\eta)$, we have a bijection

$$
\begin{aligned}
\left(\widetilde{w}^{*}\right)^{-1}(\Sigma) & \stackrel{\sim}{\rightarrow} \mathrm{JH}\left(\overline{\sigma\left(\tau_{\mathcal{S}}\right)}\right) \\
(\omega, a) & \mapsto \sigma_{(\omega, a)},
\end{aligned}
$$

where $\tau_{\mathcal{S}} \stackrel{\text { def }}{=} \tau\left(s\left(w^{*}\right)^{-1}, \mu-s\left(\widetilde{w}^{*}\right)^{-1}(0)\right)$. In what follows, $\sigma \in W^{?}\left(\bar{\rho}_{\mathcal{S}}, \tau_{\mathcal{S}}\right)$ is a Serre weight satisfying (5.1) and $V$ is a nonzero quotient of $\bar{\sigma}\left(\tau_{\mathcal{S}}\right)^{\sigma}$. We assume that for each $i \in \mathcal{J}$, there exists a subset $\Sigma_{V, i} \subseteq\left(\widetilde{w}_{i}^{*}\right)^{-1}\left(\Sigma_{0}\right)$ such that

$$
\prod_{i \in \mathcal{J}} \Sigma_{V, i} \stackrel{\sim}{\rightarrow} \mathrm{JH}(V)
$$




$$
(\omega, a) \mapsto \sigma_{(\omega, a)}=F\left(\mathfrak{T r}_{\mu}(s \omega, a)\right)
$$

is a bijection. All representations we consider below satisfy this assumption.

In the following lemmas, we use a gluing procedure to show that $M_{\infty}(V)$ is cyclic in cases where the set $W^{?}\left(\bar{\rho}_{\mathcal{S}}, \tau_{\mathcal{S}}\right)$ is of increasing complexity. In Figures $3-7$, we give a pictorial realization of the gluing procedure employed in the proofs of Lemma 5.1.4-5.1.7, respectively.

Lemma 5.1.4. Suppose that for each $i \in \mathcal{J}$, either $\ell\left(\widetilde{w}_{i}^{*}\right)>1$ or $\Sigma_{V, i} \subset\{(\omega$, $\left.1),(0,0),\left(\varepsilon_{1}, 0\right),\left(\varepsilon_{2}, 0\right)\right\}$ for some $\omega \in\left\{0, \varepsilon_{1}, \varepsilon_{2}\right\}$. Then $M_{\infty}(V)$ is a cyclic $R_{\infty}\left(\tau_{\mathcal{S}}\right)$-module.

Proof. Note that by condition (5.1), one has $(\omega, 1) \in \Sigma_{V, i}$ whenever $\ell\left(\widetilde{w}_{i}^{*}\right) \leqslant 1$. In this case, we assume, without loss of generality, that $(\omega, 0) \in \Sigma_{V, i}$ (because the quotient of a cyclic module is cyclic). Let $\bar{\Sigma}_{V, i}$ be the image of $\Sigma_{V, i}$ in $\underline{\Lambda}_{W}$ under the natural projection. We proceed by induction on

$$
n=\#\left\{i \in \mathcal{J}: \ell\left(\widetilde{w}_{i}^{*}\right) \leqslant 1 \text { and } \# \bar{\Sigma}_{V, i}=3\right\} .
$$

If $n=0$, then a casewise check shows that there exists $\widetilde{w}^{\prime} \in \operatorname{Adm}(\eta)$ such that for all $i \in \mathcal{J}, \ell\left(\widetilde{w}_{i}^{\prime}\right)>1$ and $\Sigma_{V, i} \subset \Sigma_{\left(\widetilde{w}_{i}^{\prime}\right)^{-1}}$. For example, if $\Sigma_{V, i} \subset\{(0,1),(0$, $\left.0),\left(\varepsilon_{1}, 0\right)\right\}$, then one takes $\widetilde{w}_{i}^{\prime}=\beta \alpha t_{1}$. Let $\tau_{\mathcal{S}}^{\prime}$ be $\tau_{\mathcal{S}}\left(s\left(w^{\prime}\right)^{-1}, \mu+s\left(\widetilde{w}^{\prime}\right)^{-1}(0)\right)$ where $w^{\prime} \in \underline{W}$ is the image of $\widetilde{w}^{\prime}$. Then $M_{\infty}(V)$ is a cyclic $R_{\infty}\left(\tau_{\mathcal{S}}^{\prime}\right)$-module by Lemma 5.1.3. Hence, $M_{\infty}(V)$ is a cyclic $R_{\infty}$-module, and, thus, it is a cyclic $R_{\infty}\left(\tau_{\mathcal{S}}\right)$-module as well.

Suppose now that $n>0$ so that there is an $i^{\prime} \in \mathcal{J}$ such that $\# \bar{\Sigma}_{V_{i^{\prime}}}=3$ and $\ell\left(\widetilde{w}_{i^{\prime}}^{*}\right) \leqslant 1$. By Theorem 4.1.9 and Propositions 4.1.1 and 4.1.2, there are quotients $V^{1}$ and $V^{2}$ of $V$ satisfying (5.4) with the following additional properties:

(1) $\Sigma_{V, i^{\prime}}=\Sigma_{V^{1}, i^{\prime}} \cup \Sigma_{V^{2}, i^{\prime}}$,

(2) $(\omega, 0) \in \Sigma_{V^{1}, i^{\prime}} \cap \Sigma_{V^{2}, i^{\prime}}$,

(3) $\# \bar{\Sigma}_{V^{1}, i^{\prime}}, \# \bar{\Sigma}_{V^{2}, i^{\prime}}=2$, and

(4) $\Sigma_{V^{j}, i}=\Sigma_{V, i}$ for all $i \neq i^{\prime}$ and $j=1,2$.

For example, if $\Sigma_{V, i^{\prime}}=\left\{(0,1),(0,0),\left(\varepsilon_{1}, 0\right),\left(\varepsilon_{2}, 0\right)\right\}$, then one takes $\Sigma_{V^{1}, i^{\prime}}=$ $\left\{(0,1),(0,0),\left(\varepsilon_{1}, 0\right)\right\}$ and $\Sigma_{V^{2}, i^{\prime}}=\left\{(0,1),(0,0),\left(\varepsilon_{2}, 0\right)\right\}$ (cf. Figure 3$)$.

Let $V^{3}$ be the quotient of $V^{1}$ and $V^{2}$ satisfying (5.4) such that $\Sigma_{V^{3}, i}=\Sigma_{V^{1}, i} \cap$ $\Sigma_{V^{2}, i}$ for all $i$. By the inductive hypothesis, $M_{\infty}^{\prime}\left(V^{1}\right), M_{\infty}^{\prime}\left(V^{2}\right)$, and $M_{\infty}^{\prime}\left(V^{3}\right)$ are cyclic. Let $I_{j}$ be $\operatorname{Ann}_{R_{\infty}^{\prime}\left(\tau_{\mathcal{S}}\right)} M_{\infty}^{\prime}\left(V^{j}\right)$ for $j=1,2,3$. By (1), we have an injection 
$V \hookrightarrow V^{1} \oplus V^{2}$. In fact, this injection lands in $V^{1} \times_{V^{3}} V^{2}$. Comparing lengths, we see that the injection $V \hookrightarrow V^{1} \times_{V^{3}} V^{2}$ is in fact an isomorphism, which, using the exactness of $M_{\infty}$ (see Definition 3.5.1), gives the first isomorphism below:

$$
\begin{aligned}
M_{\infty}^{\prime}(V) & \cong M_{\infty}^{\prime}\left(V^{1}\right) \times_{M_{\infty}^{\prime}\left(V^{3}\right)} M_{\infty}^{\prime}\left(V^{2}\right) \\
& \cong R_{\infty}^{\prime}\left(\tau_{\mathcal{S}}\right) / I_{1} \times_{R_{\infty}^{\prime}\left(\tau_{\mathcal{S}}\right) / I_{3}} R_{\infty}^{\prime}\left(\tau_{\mathcal{S}}\right) / I_{2} \\
& \cong R_{\infty}^{\prime}\left(\tau_{\mathcal{S}}\right) / I_{1} \times_{R_{\infty}^{\prime}\left(\tau_{\mathcal{S}}\right) /\left(I_{1}+I_{2}\right)} R_{\infty}^{\prime}\left(\tau_{\mathcal{S}}\right) / I_{2} \\
& \cong R_{\infty}^{\prime}\left(\tau_{\mathcal{S}}\right) /\left(I_{1} \cap I_{2}\right) .
\end{aligned}
$$

The second isomorphism follows from Lemma 3.6.2. The third isomorphism follows from the fact that $I_{1}+I_{2}=I_{3}$ by Theorem 3.6.4, Table 3, and Lemmas 3.6.12 and 3.6.16(3.19). We deduce in particular that $M_{\infty}^{\prime}(V)$ is cyclic.

We remind the reader that our parametrization of Serre weights $\sigma_{(\omega, a)}$ is 'centered at $\bar{\rho}_{\mathcal{S}}$ '. Thus, $\sigma_{(\omega, a)} \in W^{?}\left(\bar{\rho}_{\mathcal{S}}\right)$ exactly when $(\omega, a) \in r(\Sigma)$, and $\sigma_{(\omega, a)} \in \mathrm{JH}\left(\overline{\sigma\left(\tau_{\mathcal{S}}\right)}\right)$ exactly when $(\omega, a) \in\left(\widetilde{w}^{*}\right)^{-1}(\Sigma)$.

LEMMA 5.1.5. Suppose that for all $i \in \mathcal{J}$, either $\ell\left(\widetilde{w}_{i}^{*}\right)>1$ or

$$
\Sigma_{V, i} \subset\left(\widetilde{w}_{i}^{*}\right)^{-1}\left(\Sigma_{0} \backslash\left\{\left(v_{1}, 0\right),\left(v_{2}, 1\right),\left(v_{3}, 0\right)\right\}\right),
$$

where $\left(v_{1}, v_{2}, v_{3}\right)$ is $\left(\varepsilon_{1}-\varepsilon_{2}, \varepsilon_{1}, \varepsilon_{1}+\varepsilon_{2}\right),\left(\varepsilon_{2}-\varepsilon_{1}, \varepsilon_{2}, \varepsilon_{1}+\varepsilon_{2}\right)$, or $\left(\varepsilon_{1}-\varepsilon_{2}, 0\right.$, $\left.\varepsilon_{2}-\varepsilon_{1}\right)$. Then $M_{\infty}(V)$ is a cyclic $R_{\infty}\left(\tau_{\mathcal{S}}\right)$-module.

Proof. We note that $\#\left(\Sigma_{V, i} \cap r\left(\Sigma_{0}\right)\right) \leqslant 5$ for all $i \in \mathcal{J}$. We proceed by induction on $n=\#\left\{i \in \mathcal{J}: \#\left(\Sigma_{V, i} \cap r\left(\Sigma_{0}\right)\right)=5\right\}$. There is a unique $\widetilde{w}_{i}^{\prime} \in \operatorname{Adm}(2,1,0)$ such that

$$
\left(\widetilde{w}_{i}^{*}\right)^{-1}\left(\Sigma_{0} \backslash\left\{\left(v_{1}, 0\right),\left(v_{2}, 1\right),\left(v_{3}, 0\right)\right\}\right)=\left(\widetilde{w}_{i}^{*}\right)^{-1}\left(\Sigma_{0}\right) \cap\left(\widetilde{w}_{i}^{\prime}\right)^{-1}\left(\Sigma_{0}\right) .
$$

One can check that if \#(( $\left.\left.\widetilde{w}_{i}^{*}\right)^{-1}\left(\Sigma_{0} \backslash\left\{\left(v_{1}, 0\right),\left(v_{2}, 1\right),\left(v_{3}, 0\right)\right\}\right) \cap r\left(\Sigma_{0}\right)\right) \leqslant 4$, then one of $\ell\left(\widetilde{w}_{i}^{*}\right)$ and $\ell\left(\widetilde{w}_{i}^{\prime}\right)$ is strictly greater than one. If we change the type $\tau_{\mathcal{S}}$ so that $\widetilde{w}_{i}^{*}$ is replaced by $\widetilde{w}_{i}^{\prime}$, but $\widetilde{w}_{i^{\prime}}^{*}$ are unchanged for $i^{\prime} \neq i$, there is still a surjection $\bar{\sigma}\left(\tau_{\mathcal{S}}\right)^{\sigma} \rightarrow V$ by (5.5) and Theorem 4.1.9. We can therefore assume, without loss of generality, that for each $i$, either $\ell\left(\widetilde{w}_{i}^{*}\right)>1$ or $\#\left(\left(\widetilde{w}_{i}^{*}\right)^{-1}\left(\Sigma_{0} \backslash\left\{\left(v_{1}\right.\right.\right.\right.$, $\left.\left.\left.0),\left(v_{2}, 1\right),\left(v_{3}, 0\right)\right\}\right) \cap r\left(\Sigma_{0}\right)\right)=5$.

Suppose that $n=0$. If $\ell\left(\widetilde{w}_{i}^{*}\right) \leqslant 1$ for some $i \in \mathcal{J}$, then $\#\left(\left(\widetilde{w}_{i}^{*}\right)^{-1}\left(\Sigma_{0} \backslash\left\{\left(v_{1}\right.\right.\right.\right.$, $\left.\left.\left.0),\left(v_{2}, 1\right),\left(v_{3}, 0\right)\right\}\right) \cap r\left(\Sigma_{0}\right)\right)=5$ by assumption. On the other hand, $\Sigma_{V, i} \cap$ $r\left(\Sigma_{0}\right)<5$, which implies that $\Sigma_{V, i} \cap r\left(\Sigma_{0}\right) \subset\left\{(\omega, 1),(0,0),\left(\varepsilon_{1}, 0\right),\left(\varepsilon_{2}, 0\right)\right\}$ where $\omega=0, \varepsilon_{1}$, or $\varepsilon_{2}$ by considerations of submodule structure. Hence, there is a quotient $V^{\prime}$ of $V$ such that $V^{\prime}$ is of the form in Lemma 5.1.4 and the induced 
map $M_{\infty}(V) \rightarrow M_{\infty}\left(V^{\prime}\right)$ is an isomorphism by exactness of $M_{\infty}$ and Theorem 3.5.2. We are then done by Lemma 5.1.4.

Suppose now that $n>0$. Suppose that $\#\left(\Sigma_{V, i^{\prime}} \cap r\left(\Sigma_{0}\right)\right)=5$ for some $i^{\prime} \in \mathcal{J}$. This, in particular, implies that $\#\left(\Sigma_{V, i^{\prime}}\right)=6$, by consideration of submodule structure given in Theorem 4.1.9. Then $V$ has a unique quotient $V^{1}$ such that $\Sigma_{V^{1}, i}=\Sigma_{V, i}$ if $i \neq i^{\prime}$ and $\Sigma_{V, i^{\prime}}^{1}=\Sigma_{V, i^{\prime}} \backslash\left\{\left(\widetilde{w}_{i^{\prime}}^{*}\right)^{-1}\left(v_{4}, 1\right)\right\}$ where $v_{4}=0, \varepsilon_{1}$, or $\varepsilon_{2}$ and $v_{4} \neq v_{2}$ (cf. Figure 4 for an example in the particular case where $\tilde{w}_{i^{\prime}}=t_{1}$ ). Then $M_{\infty}\left(V^{1}\right)$ is cyclic by the induction hypothesis. There is also a submodule $V^{2} \subset V$ such that $\Sigma_{V^{2}, i}=\Sigma_{V, i}$ if $i \neq i^{\prime}$ and $\# \Sigma_{V^{2}, i^{\prime}}=\#\left(\Sigma_{V^{2}, i^{\prime}} \cap r\left(\Sigma_{0}\right)\right)=2$ (cf. Figure 4; note also that $\left(\widetilde{w}_{i^{\prime}}^{*}\right)^{-1}\left(v_{4}, 1\right) \in \Sigma_{V^{2}, i^{\prime}}$ since soc $V^{2} \subseteq$ soc $V$ ). One can check that $M_{\infty}\left(V^{2}\right)$ is cyclic by the inductive hypothesis. Then letting $M^{\prime \prime}=$ $\operatorname{ker}\left(M_{\infty}(V) \rightarrow M_{\infty}\left(V^{1}\right)\right)$ and $M^{\prime}=M_{\infty}\left(V^{2}\right)$, [EGS15, Lemma 10.1.13] implies that $M_{\infty}(V)$ is cyclic.

Lemma 5.1.6. Suppose that for all $i \in \mathcal{J}$, either $\ell\left(\widetilde{w}_{i}\right)>1$ or $\Sigma_{V, i} \subset$ $\left(\widetilde{w}_{i}^{*}\right)^{-1}\left(\Sigma_{0} \backslash\{(\nu, 0)\}\right)$ where $v$ is $\varepsilon_{1}-\varepsilon_{2}, \varepsilon_{2}-\varepsilon_{1}$, or $\varepsilon_{1}+\varepsilon_{2}$. Then $M_{\infty}(V)$ is a cyclic $R_{\infty}\left(\tau_{\mathcal{S}}\right)$-module.

In the setting of Lemma 5.1.6, note that when $\ell\left(\widetilde{w}_{i}\right) \leqslant 1$, the condition $\Sigma_{V, i} \subset$ $\left(\widetilde{w}_{i}^{*}\right)^{-1}\left(\Sigma_{0} \backslash\{(\nu, 0)\}\right), v \in\left\{\varepsilon_{1}-\varepsilon_{2}, \varepsilon_{2}-\varepsilon_{1}, \varepsilon_{1}+\varepsilon_{2}\right\}$ is equivalent to $\# \Sigma_{V, i}<9$.

Proof. We proceed by induction on

$$
\begin{aligned}
& n=\#\left\{i \in \mathcal{J}: \ell\left(\widetilde{w}_{i}^{*}\right) \leqslant 1 \text { and } \Sigma_{V, i} \not \subset\left(\tilde{w}_{i}^{*}\right)^{-1}\left(\Sigma_{0} \backslash\left\{\left(v_{1}, 0\right),\left(v_{2}, 1\right),\left(v_{3}, 0\right)\right\}\right)\right. \text { for } \\
&\left(v_{1}, v_{2}, v_{3}\right)=\left(\varepsilon_{1}-\varepsilon_{2}, \varepsilon_{1}, \varepsilon_{1}+\varepsilon_{2}\right),\left(\varepsilon_{2}-\varepsilon_{1}, \varepsilon_{2}, \varepsilon_{1}+\varepsilon_{2}\right), \\
&\text { and } \left.\left(\varepsilon_{1}-\varepsilon_{2}, 0, \varepsilon_{2}-\varepsilon_{1}\right)\right\} .
\end{aligned}
$$

The case $n=0$ is covered by Lemma 5.1.5. Suppose that $n>0$ and that $i^{\prime} \in \mathcal{J}$ with $\ell\left(\widetilde{w}_{i^{\prime}}^{*}\right) \leqslant 1$ and

$$
\Sigma_{V, i^{\prime}} \not \subset\left(\widetilde{w}_{i^{\prime}}^{*}\right)^{-1}\left(\Sigma_{0} \backslash\left\{\left(v_{1}, 0\right),\left(v_{2}, 1\right),\left(v_{3}, 0\right)\right\}\right)
$$

for $\left(\nu_{1}, \nu_{2}, \nu_{3}\right) \in\left\{\left(\varepsilon_{1}-\varepsilon_{2}, \varepsilon_{1}, \varepsilon_{1}+\varepsilon_{2}\right),\left(\varepsilon_{2}-\varepsilon_{1}, \varepsilon_{2}, \varepsilon_{1}+\varepsilon_{2}\right),\left(\varepsilon_{1}-\varepsilon_{2}, 0, \varepsilon_{2}-\varepsilon_{1}\right)\right\}$. Assume, without loss of generality, that $\Sigma_{V, i^{\prime}}=\left(\widetilde{w}_{i^{\prime}}^{*}\right)^{-1}\left(\Sigma_{0} \backslash\{(\nu, 0)\}\right)$, as the quotient of a cyclic module is again cyclic. We can further assume, without loss of generality, that $v=\varepsilon_{1}+\varepsilon_{2}$ (which implies that if $\sigma=\sigma_{(\omega, a)}$, then $\left(\omega_{i^{\prime}}, a_{i^{\prime}}\right)=$ $\left.\left(\widetilde{w}_{i^{\prime}}^{*}\right)^{-1}(0,1)\right)$. Then there are quotients $V^{1}$ and $V^{2}$ of $V$ such that

(1) $\Sigma_{V, i^{\prime}}=\Sigma_{V^{1}, i^{\prime}} \cup \Sigma_{V^{2}, i^{\prime}}$,

(2) $\Sigma_{V^{j}, i}=\Sigma_{V, i}$ for $i \neq i^{\prime}$ and $j=1,2$,

(3) $\Sigma_{V^{1}, i^{\prime}} \subset\left(\widetilde{w}_{i^{\prime}}^{*}\right)^{-1}\left(\Sigma_{0} \backslash\left\{\left(\varepsilon_{1}-\varepsilon_{2}, 0\right),\left(\varepsilon_{1}, 1\right),\left(\varepsilon_{1}+\varepsilon_{2}, 0\right)\right\}\right)$, and 
(4) $\Sigma_{V^{2}, i^{\prime}} \subset\left(\widetilde{w}_{i^{\prime}}^{*}\right)^{-1}\left(\Sigma_{0} \backslash\left\{\left(\varepsilon_{2}-\varepsilon_{1}, 0\right),\left(\varepsilon_{2}, 1\right),\left(\varepsilon_{1}+\varepsilon_{2}, 0\right)\right\}\right)$

(cf. Figure 5 for an example when $\widetilde{w}_{i^{\prime}}=\alpha$ ). We now argue by induction as in the proof of Lemma 5.1.4 using Theorem 3.6.4 and Lemmas 3.6.14, 3.6.16(3.17), and 3.6.16(3.18). Note that the analogue of Lemma 3.6.16 for the shapes $\beta$ and $\gamma$ hold symmetrically (see Remark 3.6.5).

LEMMA 5.1.7. With $V$ as described before Lemma 5.1.4, $M_{\infty}(V)$ is a cyclic $R_{\infty}\left(\tau_{\mathcal{S}}\right)$-module

Proof. We proceed by induction on

$$
n=\#\left\{i \in \mathcal{J}: \ell\left(\widetilde{w}_{i}^{*}\right) \leqslant 1 \text { and } \# \Sigma_{V, i}=9\right\} .
$$

(In the definition of $n$, note that the condition \# $\Sigma_{V, i}=9$ can be replaced by 'for all $v \in\left\{\varepsilon_{1}-\varepsilon_{2}, \varepsilon_{2}-\varepsilon_{1}, \varepsilon_{1}+\varepsilon_{2}\right\}$, one has $\Sigma_{V, i} \not \subset\left(\widetilde{w}_{i}^{*}\right)^{-1}\left(\Sigma_{0} \backslash\{(\nu, 0)\}\right)^{\prime}$.) The case $n=0$ is covered by Lemma 5.1.6. Suppose that $n>0$. Let $i^{\prime} \in \mathcal{J}$ be such that $\ell\left(\widetilde{w}_{i^{\prime}}^{*}\right) \leqslant 1$ and $\# \Sigma_{V, i^{\prime}}=9$ (equivalently, $\ell\left(\widetilde{w}_{i^{\prime}}^{*}\right) \leqslant 1$ and $\left.\Sigma_{V, i^{\prime}}=\left(\widetilde{w}_{i^{\prime}}^{*}\right)^{-1}\left(\Sigma_{0}\right)\right)$. Then $V$ has a quotient $V^{1}$ such that $\Sigma_{V^{1}, i}=\Sigma_{V, i}$ if $i \neq i^{\prime}$ and $\Sigma_{V^{1}, i^{\prime}}=\Sigma_{V, i^{\prime}}$ $\left\{\left(\widetilde{w}_{i^{\prime}}^{*}\right)^{-1}(\nu, 0)\right\}$ where $v=\varepsilon_{1}-\varepsilon_{2}, \varepsilon_{2}-\varepsilon_{1}$, or $\varepsilon_{1}+\varepsilon_{2}$ (cf. Figure 7 for an example when $\left.\widetilde{w}_{i^{\prime}}^{*}=\gamma^{+} t_{1}\right)$. If the map $M_{\infty}(V) \rightarrow M_{\infty}\left(V^{1}\right)$ is an isomorphism, we are done. Otherwise, there is a submodule $V^{2}$ of $V$ such that $\Sigma_{V^{2}, i}=\Sigma_{V, i}$ if $i \neq i^{\prime}$, $\# \Sigma_{V^{2}, i^{\prime}}=\#\left(\Sigma_{V^{2}, i^{\prime}} \cap r\left(\Sigma_{0}\right)\right)=2$, and $\left(\widetilde{w}_{i^{\prime}}^{*}\right)^{-1}(\nu, 0) \in \Sigma_{V^{2}, i^{\prime}} \subset r\left(\Sigma_{0}\right)$. Then one argues as in the proof of Lemma 5.1.5.

Proof of Theorem 5.1.1. Lemma 5.1.7 implies that $M_{\infty}\left(\bar{\sigma}\left(\tau_{\mathcal{S}}\right)^{\sigma}\right)$ is a cyclic $\bar{R}_{\infty}(\tau)$-module. Nakayama's lemma implies that $M_{\infty}\left(\sigma\left(\tau_{\mathcal{S}}\right)^{\sigma}\right)$ is a cyclic $R_{\infty}\left(\tau_{\mathcal{S}}\right)$-module. By Theorem 3.5.2, $M_{\infty}(\sigma)$, and thus $M_{\infty}\left(\sigma\left(\tau_{\mathcal{S}}\right)^{\sigma}\right)$, is nonzero. By Theorem 3.5.3, $R_{\infty}\left(\tau_{\mathcal{S}}\right)$ is irreducible and reduced so that Definition 3.5.1(3) implies that $M_{\infty}\left(\sigma\left(\tau_{\mathcal{S}}\right)^{\sigma}\right)$ is a faithful $R_{\infty}\left(\tau_{\mathcal{S}}\right)$-module. Since a faithful cyclic module is free of rank one, we are done.

Finally, we show that the hypothesis of Theorem 5.1.1 is actually necessary.

PROPOSITION 5.1.8. Let $\tau_{\mathcal{S}}$ be a 13-generic tame type and $\sigma \stackrel{\text { def }}{=} F(\lambda) \in W^{?}\left(\bar{\rho}_{\mathcal{S}}\right.$, $\left.\tau_{\mathcal{S}}\right)$. Assume that for some $i \in \mathcal{J}$,

$$
\lambda_{\pi^{-1}(i)} \in X_{1}(T) \text { is in alcove } C_{0} \text { and } \ell\left(\widetilde{w}_{i}^{*}\right) \leqslant 1 .
$$

Then $M_{\infty}\left(\sigma\left(\tau_{\mathcal{S}}\right)^{\sigma}\right)$ is not free as an $R_{\infty}\left(\tau_{\mathcal{S}}\right)$-module.

Proof. As we will not make use of this, we only give a sketch of proof. Unlike the rest of this section, we will parametrize the weights by centering around 
$\tau_{\mathcal{S}}$ so that $\tau_{\mathcal{S}}=\tau(s, \mu)$ and $\sigma_{(\omega, a)}$ means $\sigma_{(\omega, a)} \stackrel{\text { def }}{=} F\left(\mathfrak{T r}_{\mu}(s \omega, a)\right)$. In particular, $W^{?}\left(\bar{\rho}_{\mathcal{S}}, \tau_{\mathcal{S}}\right)$ consists of $\sigma_{(\omega, a)}$ such that $(\omega, a) \in \Sigma_{\widetilde{w}^{*}}$.

We write $\sigma=\sigma_{(\omega, a)}$, so $(\omega, a) \in \Sigma$. For each $i$ satisfying (5.6), we have $a_{i}=0$. If moreover $\left(\omega_{i}, a_{i}\right) \in \Sigma_{0}^{\text {inn }}$, we set $\left(\omega_{i}^{\prime}, a_{i}^{\prime}\right)=\left(\omega_{i}, 1\right)$, otherwise we set $\left(\omega_{i}^{\prime}, a_{i}^{\prime}\right)$ to be one of the two elements in $\Sigma_{0}$ that are adjacent to $\left(\omega_{i}, a_{i}\right)$. For $i$ not satisfying (5.6), we set $\left(\omega_{i}^{\prime}, a_{i}^{\prime}\right)=\left(\omega_{i}, a_{i}\right)$. Thus, we obtain a weight $\sigma^{\prime}=\sigma_{\left(\omega^{\prime}, a^{\prime}\right)}$ which satisfies the hypothesis of Theorem 5.1.1.

We now fix a saturated inclusion $\imath: \sigma\left(\tau_{\mathcal{S}}\right)^{\sigma} \hookrightarrow \sigma\left(\tau_{\mathcal{S}}\right)^{\sigma^{\prime}}$, and let $C$ denote the cokernel. Since $M_{\infty}\left(\sigma\left(\tau_{\mathcal{S}}\right)^{\sigma^{\prime}}\right) \cong R_{\infty}\left(\tau_{\mathcal{S}}\right), M_{\infty}(l)$ identifies $M_{\infty}\left(\sigma\left(\tau_{\mathcal{S}}\right)^{\sigma}\right)$ with an ideal $I(C)$ of $R_{\infty}\left(\tau_{\mathcal{S}}\right)$. Thus, we need to show that $I(C)$ is not a principal ideal, and to do so, it suffices to show that the image of $I(C)$ in $\bar{R}_{\infty}\left(\tau_{\mathcal{S}}\right)$ is not principal.

Let $V$ denote the cokernel of $l \bmod \varpi$. Using Theorem 4.1.9, we see that $V$ is multiplicity free, and $\mathrm{JH}(V) \cap W^{?}\left(\bar{\rho}_{\mathcal{S}}\right)$ consists of $\sigma_{(v, c)}$ in $(\nu, c) \in \Sigma_{\widetilde{w}^{*}} \backslash \prod_{i} \Sigma_{V^{c}, i}$, where

- if $i$ does not satisfy (5.6), $\Sigma_{V^{c}, i}=\Sigma_{\widetilde{w}_{i}^{*}}$;

- if $i$ satisfies (5.6) and $\left(\omega_{i}, a_{i}\right) \in \Sigma_{0}^{\mathrm{inn}}, \Sigma_{V^{c}, i}=\left\{\left(\omega_{i}, 0\right),\left(\mu_{1}, 1\right),\left(\mu_{2}, 1\right)\right\}$ where $\left\{\omega_{i}, \mu_{1}, \mu_{2}\right\}=\left\{0, \varepsilon_{1}, \varepsilon_{2}\right\}$; and

- if $i$ satisfies (5.6) and $\left(\omega_{i}, a_{i}\right) \notin \Sigma_{0}^{\mathrm{inn}}, \Sigma_{V^{c}, i}=\left\{\left(\omega_{i}, 0\right),\left(\omega_{i}^{\prime \prime}, 1\right)\right\}$ where $\omega_{i}^{\prime \prime}$ is such that $\left\{\omega_{i}, \omega_{i}^{\prime}, \omega_{i}^{\prime \prime}\right\}=\left\{\varepsilon_{2}-\varepsilon_{1}, 0, \varepsilon_{2}\right\},\left\{\varepsilon_{1}-\varepsilon_{2}, 0, \varepsilon_{1}\right\}$, or $\left\{\varepsilon_{1}+\varepsilon_{2}, \varepsilon_{1}, \varepsilon_{2}\right\}$.

Lemma 3.6.2 shows that $M_{\infty}(V) \cong \bar{R}_{\infty}\left(\tau_{\mathcal{S}}\right) / I(V)$, where $I(V)$ is the intersection, over $\kappa \in \mathrm{JH}(V) \cap W^{?}\left(\bar{\rho}_{\mathcal{S}}\right)$, of the ideals $\mathfrak{p}(\kappa)$ defined in Proposition 3.6.1. Note that the image of $I(C)$ in $\bar{R}_{\infty}\left(\tau_{\mathcal{S}}\right)$ is $I(V) \bar{R}_{\infty}\left(\tau_{\mathcal{S}}\right)$. Thus, we need to show $I(V) \bar{R}_{\infty}\left(\tau_{\mathcal{S}}\right)$ or, equivalently, $I(V) \bar{R}_{\infty}^{\prime}\left(\tau_{\mathcal{S}}\right)$ is not principal. Recall that there is a formally smooth map $\widehat{\bigotimes}_{i \in \mathcal{J}} \bar{R}_{\overline{\mathfrak{M}}_{\widetilde{v}}, \widetilde{w}_{i}}^{\operatorname{expl}, \nabla} \rightarrow \bar{R}_{\infty}^{\prime}\left(\tau_{\mathcal{S}}\right)$. We claim that the ideal $I(V)$ comes from $I \stackrel{\text { def }}{=} \widehat{\bigotimes}_{i}\left(\bigcap_{\left(v_{i}, c_{i}\right) \in \Sigma_{\widetilde{w}_{i}^{*}} \backslash \Sigma_{V^{c}, i}} \mathfrak{c}_{\left(v_{i}, c_{i}\right)} \bar{R}_{\bar{M}_{\widetilde{v}}, \widetilde{w}_{\tilde{v}^{-1}-i}}^{\operatorname{expl}, \nabla}\right)$. To check the claim, let $\bar{R}_{\overline{\mathfrak{M}}, \widetilde{w}}^{\operatorname{expl}, \nabla} \stackrel{\text { def }}{=} \widehat{\bigotimes}_{i \in \mathcal{J}} \bar{R}_{\overline{\mathfrak{M}}_{\widetilde{v}}, \widetilde{w}_{i}}^{\text {expl, }}$. We first check that $\bar{R} \overline{\mathfrak{M}}, \widetilde{w} / I$ has the expected cycle. To do this, we check using Table 3 that $\bar{R}_{\bar{M}_{\widetilde{v}}, \widetilde{w}_{f_{\tilde{v}}-1-i}}^{\operatorname{expl}, \nabla} / \bigcap_{\left(\nu_{i}, c_{i}\right) \in \Sigma_{\widetilde{w}_{i}^{*}} \backslash \Sigma_{V^{c}, i}} \mathfrak{c}_{\left(v_{i}, c_{i}\right)} \bar{R}_{\mathfrak{M}_{\widetilde{v}}, \widetilde{w}_{f_{\tilde{v}}-1-i}}^{\operatorname{expl}, \nabla}$ is filtered by $\bar{R}_{\mathfrak{M}_{\tilde{v}}, \widetilde{w}_{f_{\tilde{v}}-1-i}}^{\operatorname{expl}, \nabla} / \mathfrak{c}_{\left(v_{j}, c_{j}\right)}$ for $\left(v_{j}, c_{j}\right) \in \Sigma_{\widetilde{w}_{i}^{*}} \backslash \Sigma_{V^{c}, i}$ and that $\bigcap_{\left(v_{i}, c_{i}\right) \in \Sigma_{\widetilde{w}_{i}^{*} \backslash \Sigma_{V^{c}, i}}} \mathfrak{c}_{\left(v_{i}, c_{i}\right)} \bar{R}_{\bar{M}_{\widetilde{v}}, \widetilde{w}_{\tilde{v}^{-1-i}}}^{\operatorname{expl,\nabla }}$ is filtered by $\bar{R}_{\mathfrak{M}_{\tilde{v}}, \widetilde{w}_{\tilde{v}^{-1-i}}}^{\operatorname{expl}, \nabla} / \mathfrak{c}_{\left(v_{j}, c_{j}\right)}$ for $\left(v_{j}, c_{j}\right) \in \Sigma_{V^{c}, i}$. Thus, we can filter the quotient $\bar{R}_{\overline{\mathfrak{M}}, \widetilde{w}}^{\operatorname{expl}, \nabla} / I$ with factors of the form $\widehat{\otimes}_{i \in \mathcal{J}} \bar{R}_{\mathfrak{M}_{\widetilde{v}}, \widetilde{w}_{i}}^{\operatorname{expl}, \nabla} / \mathfrak{p}_{i}$, where the $\mathfrak{p}_{i}$ are minimal primes of $\bar{R}_{\overline{\mathfrak{M}}_{\widetilde{v}}, \widetilde{w}_{i}}^{\text {expl, }}$ (note that taking completion is an exact operation). As $\Gamma$ (im( $l$ 
$(\bmod \varpi)))$ decomposes as a product over $\mathcal{J}$, an inductive argument using Theorem 3.6.4 and the above filtration on $\overline{R_{\mathfrak{M}}, \widetilde{w}} / I$ shows that the cycle of $\bar{R}_{\overline{\mathfrak{M}}, \widetilde{w}}^{\operatorname{expl}, \nabla} / I$ is given by the components of $\bar{R}_{\overline{\mathfrak{M}}, \widetilde{w}}^{\exp , \nabla}$ labeled by the Serre weights corresponding to $\Gamma(\operatorname{im}(l(\bmod \varpi)))$. In order to prove the claim, we are left to show that $I$ is a radical ideal. To see this, we make the following observation: If $R, S$ are two reduced local Noetherian rings over a perfect field $k$ and $J, L$ are radical ideals of $R, S$, then $J \otimes_{k} L$ is a radical ideal of $R \otimes_{k} S$. This is because $(R \otimes S) /(J \otimes L)$ embeds into $((R / J) \otimes S) \times(R \otimes(S / L))$ (as can be seen by choosing bases of $J, L$ as $k$-vector spaces and extending them to bases of $R, S$ ), and the latter ring is reduced. Finally, we note that in the situation above, the property of being a radical ideal is preserved by completion so that $J \widehat{\otimes}_{k} L$ is also radical in $R \widehat{\otimes}_{k} S$. The claim is proven.

By Nakayama's lemma, the size of a minimal set of generators of $I$ is given by the dimension over $\mathbb{F}$ of

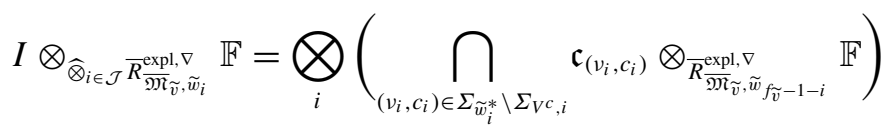

Hence, it suffices to show that for $i$ satisfying (5.6),

$$
\bigcap_{\left(v_{i}, c_{i}\right) \in \Sigma_{\widetilde{w}_{i}^{*} \backslash \Sigma_{V^{c}, i}}} \mathfrak{c}_{\left(v_{i}, c_{i}\right)} \otimes_{\bar{R}_{\overline{\mathfrak{M}}_{\tilde{v}}, \tilde{w}} \tilde{v}_{\widetilde{v}-1-i}} \mathbb{F}
$$

has dimension greater than one, which can be checked from Table 3.

5.2. Gauges for patching functors. In this subsection, we compute the image of maps between patched modules for lattices in Deligne-Lusztig representations. This can be viewed as a calculation of lattice gauges in families. The main ingredient for this section is Theorem 5.1.1, after which algebrogeometric arguments using the projection formula and the Cohen-Macaulay property prove Theorem 5.2.3. We learned these arguments from M. Emerton.

We continue to use the weak minimal patching functor setting of Section 3.5. Let $\mathcal{S}, \bar{\rho}_{\mathcal{S}}, R_{\infty}, X_{\infty}$, and $M_{\infty}$ be as in Section 5.1. Let $\tau_{\mathcal{S}}$ be $\left(\tau_{\widetilde{v}}\right)_{\widetilde{v} \in \mathcal{S}}$, where $\tau_{\widetilde{v}}$ is a 13-generic tame type. Let $X_{\infty}\left(\tau_{\mathcal{S}}\right) \stackrel{\text { def }}{=} \operatorname{Spec} R_{\infty}\left(\tau_{\mathcal{S}}\right)$. It is a normal and Cohen-Macaulay scheme by (the proof of) Theorem 3.5.3. Let $Z \subset X_{\infty}\left(\tau_{\mathcal{S}}\right)$ be the locus of points lying on two irreducible components of the special fiber of $X_{\infty}\left(\tau_{\mathcal{S}}\right)$. Note that the codimension of $Z \subset X_{\infty}\left(\tau_{\mathcal{S}}\right)$ is two. Let

$$
j: U \stackrel{\text { def }}{=} X_{\infty}\left(\tau_{\mathcal{S}}\right) \backslash Z \hookrightarrow X_{\infty}\left(\tau_{\mathcal{S}}\right)
$$


be the natural open immersion. Note that $j$ and $U$ come from pulling back the open immersion $j_{0}: U_{0} \hookrightarrow \operatorname{Spf}\left(\widehat{\bigotimes}_{\widetilde{v} \in \mathcal{S}} R_{\widetilde{v}}^{\tau_{\tilde{v}}}\right)$ via the formally smooth map $X_{\infty}\left(\tau_{\mathcal{S}}\right) \rightarrow \operatorname{Spf}\left(\widehat{\bigotimes}_{\widetilde{v} \in \mathcal{S}} R_{\widetilde{v}}^{\tau_{\tilde{v}}}\right)$, where $U_{0}$ is defined in an analogous way as $U$.

\section{LEMMA 5.2.1. The scheme $U$ is regular.}

Proof. The irreducible components of the special fiber of $X_{\infty}\left(\tau_{\mathcal{S}}\right)$ are formally smooth over $\mathbb{F}$; hence, the special fiber $\bar{U}$ of $U$ is regular by the last part of Theorem 3.5.3. The dimension of the tangent space of $U$ at a characteristic $p$ point is at most one more than the dimension of the tangent space of $\bar{U}$ at that point. By $p$-flatness, the Krull dimension of $U$ is one more than the Krull dimension of $\bar{U}$, and so $U$ is regular at characteristic $p$ points. Since the generic fiber of $U$, which is isomorphic to the generic fiber of $X_{\infty}\left(\tau_{\mathcal{S}}\right)$, is regular, $U$ is regular.

We now use the notation $j^{*}$ and $j_{*}$ which take quasicoherent sheaves on $X_{\infty}\left(\tau_{\mathcal{S}}\right)$ to those on $U$ and vice versa, respectively.

LEMMA 5.2.2. Let $\sigma, \kappa \in \mathrm{JH}\left(\overline{\sigma\left(\tau_{\mathcal{S}}\right)}\right)$ and let $\iota: \sigma\left(\tau_{\mathcal{S}}\right)^{\kappa} \hookrightarrow \sigma\left(\tau_{\mathcal{S}}\right)^{\sigma}$ be a saturated injection. For any $\theta=\bigotimes_{\widetilde{v} \in \mathcal{S}} \theta_{\widetilde{v}} \in W^{?}\left(\bar{\rho}_{\mathcal{S}}\right)$, let $m(\theta)$ be the multiplicity with which $\theta$ appears in the cokernel of $\iota$. Then the induced injection $j^{*} M_{\infty}(\iota)$ : $j^{*} M_{\infty}\left(\sigma\left(\tau_{\mathcal{S}}\right)^{\kappa}\right) \hookrightarrow j^{*} M_{\infty}\left(\sigma\left(\tau_{\mathcal{S}}\right)^{\sigma}\right)$ has image

$$
j^{*}\left(\prod_{\theta \in W^{?}\left(\bar{\rho}_{\mathcal{S}}\right)} \mathfrak{p}(\theta)^{m(\theta)} M_{\infty}\left(\sigma\left(\tau_{\mathcal{S}}\right)^{\sigma}\right)\right),
$$

where $\mathfrak{p}(\theta)=\sum_{\widetilde{v} \in \mathcal{S}} \mathfrak{p}\left(\theta_{\widetilde{v}}\right)$.

Proof. Note that $j^{*}\left(M_{\infty}\left(\sigma\left(\tau_{\mathcal{S}}\right)^{\kappa}\right)\right)$ and $j^{*}\left(M_{\infty}\left(\sigma\left(\tau_{\mathcal{S}}\right)^{\sigma}\right)\right)$ are locally free (of rank one) since they are Cohen-Macaulay of full support over the regular scheme $U$ (see Definition 3.5.1). Then the image of $j^{*} M_{\infty}(\iota)$ is $J \otimes_{\mathcal{O}_{U}} j^{*}\left(M_{\infty}\left(\sigma\left(\tau_{\mathcal{S}}\right)^{\sigma}\right)\right.$ ), where $J$ is the ideal sheaf of the Cartier divisor corresponding to the cokernel of $j^{*} M_{\infty}(\iota)$. It is easily seen that $J=j^{*}\left(\prod_{\theta \in W^{?}\left(\bar{\rho}_{\mathcal{S}}\right)} \mathfrak{p}(\theta)^{m(\theta)} R_{\infty}\left(\tau_{\mathcal{S}}\right)\right)$. Finally, we have that

$$
\begin{aligned}
& j^{*}\left(\prod_{\theta \in W^{?}\left(\bar{\rho}_{\mathcal{S}}\right)} \mathfrak{p}(\theta)^{m(\theta)} R_{\infty}\left(\tau_{\mathcal{S}}\right)\right) \otimes_{\mathcal{O}_{U}} j^{*}\left(M_{\infty}\left(\sigma\left(\tau_{\mathcal{S}}\right)^{\sigma}\right)\right) \\
& \cong j^{*}\left(\prod_{\theta \in W^{?}\left(\bar{\rho}_{\mathcal{S}}\right)} \mathfrak{p}(\theta)^{m(\theta)} R_{\infty}\left(\tau_{\mathcal{S}}\right)\right) j^{*}\left(M_{\infty}\left(\sigma\left(\tau_{\mathcal{S}}\right)^{\sigma}\right)\right)
\end{aligned}
$$




$$
=j^{*}\left(\prod_{\theta \in W^{?}\left(\bar{\rho}_{\mathcal{S}}\right)} \mathfrak{p}(\theta)^{m(\theta)} M_{\infty}\left(\sigma\left(\tau_{\mathcal{S}}\right)^{\sigma}\right)\right)
$$

where the isomorphism follows from the fact that $j^{*}\left(M_{\infty}\left(\sigma\left(\tau_{\mathcal{S}}\right)^{\sigma}\right)\right)$ is locally free.

Let $\sigma$ be as in Theorem 5.1.1 so that $M_{\infty}\left(\sigma\left(\tau_{\mathcal{S}}\right)^{\sigma}\right)$ is cyclic.

THEOREM 5.2.3. With the notation of Lemma 5.2.2, suppose further that $\sigma$ is as in Theorem 5.1.1. Then the induced injection $M_{\infty}(\iota): M_{\infty}\left(\sigma\left(\tau_{\mathcal{S}}\right)^{\kappa}\right) \hookrightarrow$ $M_{\infty}\left(\sigma\left(\tau_{\mathcal{S}}\right)^{\sigma}\right)$ has image

$$
j_{*} j^{*}\left(\prod_{\theta \in W^{?}\left(\bar{\rho}_{\mathcal{S}}\right)} \mathfrak{p}(\theta)^{m(\theta)} R_{\infty}\left(\tau_{\mathcal{S}}\right)\right) M_{\infty}\left(\sigma\left(\tau_{\mathcal{S}}\right)^{\sigma}\right) .
$$

Proof. If $\mathcal{M}$ is a Cohen-Macaulay sheaf on $X_{\infty}\left(\tau_{\mathcal{S}}\right)$, then $j_{*} j^{*} \mathcal{M}=\mathcal{M}$ since the codimension of $Z$ is two (cf. [HK04, Proposition 3.5]). Hence, the image of $M_{\infty}(\iota)$ is

$$
j_{*} j^{*}\left(\prod_{\theta \in W^{?}\left(\bar{\rho}_{\mathcal{S}}\right)} \mathfrak{p}(\theta)^{m(\theta)} M_{\infty}\left(\sigma\left(\tau_{\mathcal{S}}\right)^{\sigma}\right)\right)
$$

by Lemma 5.2.2. Since $M_{\infty}\left(\sigma\left(\tau_{\mathcal{S}}\right)^{\sigma}\right)$ is free over $R_{\infty}\left(\tau_{\mathcal{S}}\right)$, we have that

$$
\begin{aligned}
j_{*} j^{*} & \left(\prod_{\theta \in W^{?}\left(\bar{\rho}_{\mathcal{S}}\right)} \mathfrak{p}(\theta)^{m(\theta)} M_{\infty}\left(\sigma\left(\tau_{\mathcal{S}}\right)^{\sigma}\right)\right) \\
& =j_{*} j^{*}\left(\prod_{\theta \in W^{?}\left(\bar{\rho}_{\mathcal{S}}\right)} \mathfrak{p}(\theta)^{m(\theta)} R_{\infty}\left(\tau_{\mathcal{S}}\right)\right) M_{\infty}\left(\sigma\left(\tau_{\mathcal{S}}\right)^{\sigma}\right) .
\end{aligned}
$$

REMARK 5.2.4. As $j$ comes from pulling back $j_{0}$ via $X_{\infty}\left(\tau_{\mathcal{S}}\right) \rightarrow$ $\operatorname{Spf}\left(\widehat{\bigotimes}_{\widetilde{v} \in \mathcal{S}} R_{\widetilde{v}}^{\tau_{v}}\right)$ and $\mathfrak{p}(\theta)$ are ideals of $\operatorname{Spf}\left(\widehat{\bigotimes}_{\widetilde{v} \in \mathcal{S}} R_{\widetilde{v}}^{\tau_{v}}\right)$, we see that the ideal

$$
j_{*} j^{*}\left(\prod_{\theta \in W^{?}\left(\bar{\rho}_{\mathcal{S}}\right)} \mathfrak{p}(\theta)^{m(\theta)} R_{\infty}\left(\tau_{\mathcal{S}}\right)\right)=j_{0 *} j_{0}^{*}\left(\prod_{\theta \in W^{?}\left(\bar{\rho}_{\mathcal{S}}\right)} \mathfrak{p}(\theta)^{m(\theta)} \widehat{\bigotimes}_{\widetilde{v} \in \mathcal{S}} R_{\widetilde{v}}^{\tau_{v}}\right) R_{\infty}\left(\tau_{\mathcal{S}}\right)
$$

comes from an ideal in $\widehat{\bigotimes}_{\widetilde{v} \in \mathcal{S}} R_{\widetilde{v}}^{\square}$.

5.3. Global applications. In this subsection, we deduce generalizations of conjectures of Dembélé and Breuil on mod $p$ multiplicity one and lattices, respectively (see [Bre14, Conjectures B.1 and 1.2]). Theorem 5.3.4 follows immediately from Theorem 5.1.1. While Theorem 5.3.5 also follows from 
Theorem 5.2.3, it is crucial that the image of the map between two patched modules given in Theorem 5.2.3 is described by an ideal. This is far from formal and relies crucially on Theorem 5.1.1.

We use the setup in [LLHLM18, Section 7.1]. Let $F / \mathbb{Q}$ be a CM field with maximal totally real subfield $F^{+} \neq \mathbb{Q}$ and let $\Sigma_{p}^{+}$(respectively $\Sigma_{p}$ ) be the set of places of $F^{+}$(respectively of $F$ ) lying above $p$. Let $G_{/ F^{+}}$be a reductive group which is an outer form for $\mathrm{GL}_{3}$, which is quasisplit at all finite places of $F^{+}$, and which splits over $F$. Suppose that $G_{/ F^{+}}$is definite, that is, that $G\left(F_{v}^{+}\right) \cong U_{3}(\mathbb{R})$ for all $v \mid \infty$. Recall from [EGH13, Section 7.1] that $G$ admits a reductive model $\mathcal{G}$ defined over $\mathcal{O}_{F^{+}}[1 / N]$, for some $N \in \mathbb{N}$ which is prime to $p$, together with an isomorphism

$$
\iota: \mathcal{G}_{/ \mathcal{O}_{F}[1 / N]} \stackrel{\iota}{\rightarrow} \mathrm{GL}_{3 / \mathcal{O}_{F}[1 / N]}
$$

which specializes to $\iota_{w}: \mathcal{G}\left(\mathcal{O}_{F_{v}^{+}}\right) \stackrel{\sim}{\rightarrow} \mathcal{G}\left(\mathcal{O}_{F_{w}}\right) \stackrel{\iota}{\rightarrow} \operatorname{GL}_{3}\left(\mathcal{O}_{F_{w}}\right)$ for all places $w \in$ $\Sigma_{p}$ with $\left.w\right|_{F^{+}}=v$.

Let $U=U^{p} U_{p} \leqslant G\left(\mathbb{A}_{F^{+}}^{(\infty, p)}\right) \times \mathcal{G}\left(\mathcal{O}_{F^{+}, p}\right)$ be a compact open subgroup. If $W$ is a finite $\mathcal{O}$-module endowed with a continuous action of $U$, we write $S(U, W)$ to denote the space of algebraic automorphic forms with coefficients in $W$ :

$$
\begin{aligned}
S(U, W) \stackrel{\text { def }}{=}\left\{f: G\left(F^{+}\right) \backslash G\left(\mathbb{A}_{F^{+}}^{\infty}\right) \rightarrow W \mid f(g u)\right. \\
\left.=u^{-1} f(g) \forall g \in G\left(\mathbb{A}_{F^{+}}^{\infty}\right), u \in U\right\} .
\end{aligned}
$$

We define

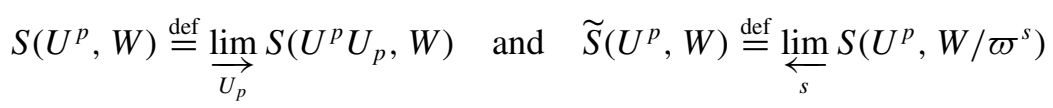

where in the first limit, the subgroups $U_{p} \leqslant \mathcal{G}\left(\mathcal{O}_{F^{+}, p}\right)$ run over the compact open neighborhoods of $1 \in \mathcal{G}\left(\mathcal{O}_{F^{+}, p}\right)$.

For $U$ as above, let $\mathcal{P}_{U}$ be the set of finite places $w$ in $F$ whose restriction $\left.v \stackrel{\text { def }}{=} w\right|_{F^{+}}$is a place that splits in $F$ and at which $U$ is unramified. Let $\mathcal{P} \subset \mathcal{P}_{U}$ be a subset of finite complement. Then the universal Hecke algebra $\mathbb{T}_{\mathcal{P}}=\mathcal{O}\left[T_{w}^{(i)}\right.$, $w \in \mathcal{P}, 0 \leqslant i \leqslant n]$ on $\mathcal{P}$ acts naturally on $S(U, W)$. Let $\bar{r}: G_{F} \rightarrow \mathrm{GL}_{3}(\mathbb{F})$ be a continuous Galois representation. We let $\mathfrak{m} \subseteq \mathbb{T}_{\mathcal{P}}$ be the maximal ideal which is the kernel of the system of Hecke eigenvalues $\bar{\alpha}: \mathbb{T}_{\mathcal{P}} \rightarrow \mathbb{F}$ associated with $\bar{r}$, that is, $\bar{\alpha}$ satisfies

$$
\operatorname{det}\left(1-\bar{r}^{\vee}\left(\operatorname{Frob}_{w}\right) X\right)=\sum_{j=0}^{3}(-1)^{j}\left(\mathbf{N}_{F / \mathbb{Q}}(w)\right)^{\left(\begin{array}{l}
j \\
2
\end{array}\right)} \bar{\alpha}\left(T_{w}^{(j)}\right) X^{j}
$$

for all $w$ as above. Then we say that $\bar{r}$ is automorphic if $S(U, W)_{\mathfrak{m}}$ is nonzero for some $U$ and $W$. 
For technical reasons, we choose a place $v_{1}$ of $F^{+}$as in [CEG+16, Section 2.3]. We now fix $U^{p} \leqslant G\left(\mathbb{A}_{F^{+}}^{(\infty, p)}\right)$ to be the subgroup with:

(1) $\left(U^{p}\right)_{v}=\mathcal{G}\left(\mathcal{O}_{F_{v}^{+}}\right)$for all finite places $v$ of $F^{+}$which split in $F$ and do not belong to $\Sigma_{p}^{+} \cup\left\{v_{1}\right\}$;

(2) $\left(U^{p}\right)_{v_{1}}$ is the preimage of the upper triangular matrices under the map

$$
\mathcal{G}\left(\mathcal{O}_{F_{v_{1}}^{+}}\right) \rightarrow \mathcal{G}\left(k_{v_{1}}\right) \stackrel{\iota_{\tau_{1}}}{\rightarrow} \mathrm{GL}_{3}\left(k_{\widetilde{v}_{1}}\right)
$$

(3) $\left(U^{p}\right)_{v}$ is hyperspecial maximal compact in $G\left(F_{v}^{+}\right)$if $v$ is inert in $F$.

Let $\bar{r}$ be an automorphic Galois representation. Let $\Sigma_{0}^{+}$denote the set of finite places of $F^{+}$which are the restriction of the finite places of $F$ away from $p$ where $\bar{r}$ ramifies. For each $v \in \Sigma_{0}^{+}$, we let $\tau_{\widetilde{v}}$ be the minimally ramified type in the sense of [CHT08, Definition 2.4.14] corresponding to $\left.\bar{r}\right|_{G_{F_{\tilde{v}}}}$ and $\sigma\left(\tau_{\widetilde{v}}\right)$ be the $\mathrm{GL}_{3}\left(\mathcal{O}_{F_{\tilde{v}}}\right)$-representation over $E$ associated with it (cf. the beginning of Section 5.3.1). We write $\sigma\left(\tau_{v}\right) \stackrel{\text { def }}{=} \sigma\left(\tau_{\widetilde{v}}\right) \circ \iota_{\widetilde{v}}$, which is a $\mathcal{G}\left(\mathcal{O}_{F_{v}^{+}}\right)$-representation independent of the choice of $\widetilde{v} \mid v$. For each $v \in \Sigma_{0}^{+}$, fix a $\mathcal{O}$-lattice $\sigma\left(\tau_{v}\right)^{\circ}$ in $\sigma\left(\tau_{v}\right)$ and let $W_{\Sigma_{0}^{+}}$be $\otimes_{v \in \Sigma_{0}^{+}} \sigma\left(\tau_{v}\right)^{\circ}$.

We let $\mathbb{T}^{\text {univ }}$ denote the abstract Hecke algebra over $\mathcal{O}$ generated by the formal variables $T_{w}^{(j)}$, where $w$ runs over the finite places of $F$ such that $\left.w\right|_{F+}$ is split in $F$ and $\left.w\right|_{F^{+}} \notin \Sigma_{0}^{+} \cup \Sigma_{p}^{+} \cup\left\{v_{1}\right\}$, and by $T_{v_{1}}^{(j)}$ for $j=1,2,3$. For a $G\left(\mathbb{A}_{F^{+}}^{(\infty, p)}\right)$ module $V$ over $\mathcal{O}, \mathbb{T}^{\text {univ }}$ acts naturally on $S\left(U^{p} U_{p}, W\right), S\left(U^{p}, W\right)$, and $\widetilde{S}\left(U^{p}\right.$, $W$ ) where $W=W_{\Sigma_{0}^{+}} \otimes V$ (cf. [CEG+16, Section 2.3]), and we let $\mathfrak{m} \subseteq \mathbb{T}^{\text {univ }}$ be the maximal ideal as before. We will now assume that $S(U, W)_{\mathfrak{m}}$ is nonzero for some choice of $V$ above. In fact, one can show, by the proof of Proposition 6.0.2, that this is a consequence of the hypothesis that $\bar{r}$ is automorphic.

In the remainder of this section, we let $\bar{r}: G_{F} \rightarrow \mathrm{GL}_{3}(\mathbb{F})$ be an automorphic Galois representation that satisfies the Taylor-Wiles conditions in the sense of [LLHLM18, Definition 7.3]. We assume furthermore that

(i) the extension $F / F^{+}$is unramified at all finite places;

(ii) (split ramification) if $\bar{r}: G_{F} \rightarrow \mathrm{GL}_{3}(\mathbb{F})$ is ramified at a place $w$ of $F$, then $v=\left.w\right|_{F^{+}}$splits as $w w^{c}$

(iii) $p$ is unramified in $F^{+}$and all places in $F^{+}$above $p$ split in $F$; and

(iv) $\left.\bar{r}\right|_{G_{F w}}$ is semisimple for all $w \in \Sigma_{p}$. 
For each $v \in \Sigma_{p}^{+}$, we choose a place $\widetilde{v} \mid v$ of $F$ and let $\mathcal{S}$ be the set $\left\{\widetilde{v} \mid v \in \Sigma_{p}^{+}\right\}$. Let $\bar{\rho}_{\widetilde{v}}$ be $\left.\bar{r}\right|_{G_{F_{v}}}$ and $\bar{\rho}_{\mathcal{S}}$ be $\left(\bar{\rho}_{\widetilde{v}}\right)_{\widetilde{v} \in \mathcal{S}}$. We set $K \stackrel{\text { def }}{=} \prod_{\widetilde{v} \in \mathcal{S}} \operatorname{GL}_{3}\left(\mathcal{O}_{F_{\widetilde{v}}}\right)$. Let $\widetilde{M}_{\infty}$ be the weak minimal patching functor for $\bar{r}$ in the sense of [LLHLM18, Definition 7.11] constructed in [LLHLM18, Proposition 7.15]. As in Section 3.5, we let $M_{\infty}$ be $\widetilde{M}_{\infty} \circ \prod_{\widetilde{v} \in \mathcal{S}} \iota \widetilde{v}$, which is a weak minimal patching functor for $\bar{\rho}_{\mathcal{S}}$ by Proposition 3.5.14.

Along with the construction of $\tilde{M}_{\infty}$ (cf. [CEG+16, Section 2.8], [Le18, Section 4.2], and [LLHLM18, Section 7.3]), one has a ring homomorphism $R_{\infty}=\widehat{\bigotimes}_{\widetilde{v} \in \mathcal{S}} R_{\widetilde{v}}^{\square} \llbracket x_{1}, \ldots, x_{h} \rrbracket \rightarrow \mathbb{T}_{U^{p} \mathcal{G}\left(\mathcal{O}_{F^{+}, p}\right)}^{\text {univ }}\left(W_{\Sigma_{0}^{+}}\right)_{\mathfrak{m}}$, where $\mathbb{T}_{U^{p} \mathcal{G}\left(\mathcal{O}_{F^{+}, p}\right)}^{\text {univ }}\left(W_{\Sigma_{0}^{+}}\right)$ is the image of $\mathbb{T}^{\text {univ }}$ in

$$
\operatorname{End}_{\mathcal{O}}\left(S\left(U^{p} \mathcal{G}\left(\mathcal{O}_{F^{+}, p}\right), W_{\Sigma_{0}^{+}}\right)\right) .
$$

We also write $\mathfrak{m}$ for the pullback in $R_{\infty}$ of the maximal ideal of $\mathbb{T}_{U^{p} \mathcal{G}\left(\mathcal{O}_{F^{+}, p}\right)}^{\text {univ }}\left(W_{\Sigma_{0}^{+}}\right)_{\mathfrak{m}}$. Then if $\bar{W}_{\Sigma_{p}^{+}}$is a smooth, finite-dimensional $\mathcal{G}\left(\mathcal{O}_{F^{+}, p}\right)$ representation over $\mathbb{F}$, one has

$$
\left(\tilde{M}_{\infty}\left(\bar{W}_{\Sigma_{p}^{+}}\right) / \mathfrak{m}\right)^{\vee}=S\left(U^{p} \mathcal{G}\left(\mathcal{O}_{F^{+}, p}\right), \bar{W}_{\Sigma_{0}^{+}} \otimes \bar{W}_{\Sigma_{p}^{+}}^{\vee}\right)[\mathfrak{m}]
$$

where ${ }^{\vee}$ denotes Pontrjagin duals (cf. [Le18, Theorem 4.1.5]).

5.3.1. Automorphy lifting. Recall from Proposition 2.2.6 that if $\widetilde{v} \in \mathcal{S}$ and $\tau_{\widetilde{v}}$ is a 1-generic tame inertial type for $I_{F_{\tilde{v}}}$, we defined a $\mathrm{GL}_{3}\left(\mathcal{O}_{F_{\widetilde{v}}}\right)$-representation $\sigma\left(\tau_{\widetilde{v}}\right)$ over $E$ corresponding to $\tau_{\widetilde{v}}$ by results toward inertial local Langlands. We again let $\sigma\left(\tau_{\mathcal{S}}\right)$ be $\bigotimes_{\tilde{v} \in \mathcal{S}} \sigma\left(\tau_{\widetilde{v}}\right)$.

THEOREM 5.3.1. Let $r: G_{F} \rightarrow \mathrm{GL}_{3}(E)$ be an absolutely irreducible Galois representation such that

(1) for all places $w \in \Sigma_{p},\left.\bar{r}\right|_{I_{F}}$ is semisimple and 10-generic;

(2) $r$ is unramified almost everywhere and satisfies $r^{c} \cong r^{\vee} \epsilon^{-2}$;

(3) for all places $\tilde{v} \in \mathcal{S}$, the representation $\left.r\right|_{G_{F_{\tilde{v}}}}$ is potentially crystalline, with parallel Hodge-Tate weights $(2,1,0)$ and with tame inertial type $\tau_{\widetilde{v}}$ (see [LLHLM18, Definition 2.1]);

(4) $\bar{r}$ satisfies the Taylor-Wiles conditions as above and $\bar{r}$ has split ramification; and

(5) $\bar{r} \cong \bar{r}_{l}(\pi)$ for a regular algebriac conjugate self dual cuspidal (RACSDC) representation $\pi$ of $\mathrm{GL}_{3}\left(\mathbb{A}_{F}\right)$ with trivial infinitesimal character such that $\bigotimes_{\widetilde{v} \in \mathcal{S}} \sigma\left(\tau_{\widetilde{v}}\right)$ is a $K$-type for $\bigotimes_{\widetilde{v} \in \mathcal{S}} \pi_{\widetilde{v}}$. 
Then $r$ is automorphic in the sense of [LLHLM18, Section 7.2].

Proof. Given Theorems 5.3.3 and 3.5.3, the proof of [LLHLM18, Theorem 7.4] goes through unchanged.

REMARK 5.3.2. Compared to [LLHLM18, Theorem 7.4], we relaxed the hypothesis that $p$ splits completely to $p$ being unramified. However, we also assumed that $\bar{r}$ is semisimple at all places above $p$. The reason is that we only established the connectedness of the generic fiber of $R_{\bar{\rho}}^{\tau}$ when $\bar{\rho}$ is semisimple (though we do know it for non-semisimple $\bar{\rho}$ in the case that all shapes have length $\geqslant 2$ ). In work in progress, we will establish a counterpart of [LLHLM18, Theorem 7.4] for non-semisimple representations. This will allow us to remove the semisimplicity hypothesis in a manner similar to [LLHLM18, Theorem 7.4].

5.3.2. The Serre weight conjecture. Let $\bar{r}: G_{F} \rightarrow \mathrm{GL}_{3}(\mathbb{F})$ be as in the beginning of Section 5.3. Recall that for each $v \in \Sigma_{p}^{+}$, we chose a place $\widetilde{v} \mid v$ of $F$ and set $\mathcal{S}$ to be the set $\left\{\widetilde{v} \mid v \in \Sigma_{p}^{+}\right\}$. Furthermore, we let $\bar{\rho}_{\widetilde{v}}$ be $\left.\bar{r}\right|_{G_{F_{v}}}$ and $\bar{\rho}_{\mathcal{S}}$ be $\left(\bar{\rho}_{\widetilde{v}}\right)_{\widetilde{v} \in \mathcal{S}}$. Recall that $K$ is the product $\prod_{\widetilde{v} \in \mathcal{S}} \mathrm{GL}_{3}\left(\mathcal{O}_{F_{\widetilde{v}}}\right)$. Let $W(\bar{r})$ be the set of irreducible $K$-representations $\sigma$ over $\mathbb{F}$ such that

$$
S\left(U^{p} \mathcal{G}\left(\mathcal{O}_{F^{+}, p}\right), \bar{W}_{\Sigma_{0}^{+}} \otimes\left(\sigma^{\vee} \circ \prod_{\widetilde{v} \in \mathcal{S}} \iota \widetilde{v}\right)\right)_{\mathfrak{m}} \neq 0 .
$$

We have the following version of the weight part of Serre's conjecture.

THEOREM 5.3.3. Let $\bar{r}: G_{F} \rightarrow \mathrm{GL}_{3}(\mathbb{F})$ be a continuous Galois representation, satisfying the Taylor-Wiles conditions. Assume that $\left.\bar{\rho}_{\widetilde{v}} \stackrel{\text { def }}{=} \bar{r}\right|_{G_{F}}$ is semisimple and 10-generic for all $\widetilde{v} \in \mathcal{S}$, that $\bar{r}$ is automorphic, and that $\bar{r}$ has split ramification. Then $W(\bar{r}) \circ \prod_{\widetilde{v} \in \mathcal{S}} \iota \widetilde{v}=W^{?}\left(\bar{\rho}_{\mathcal{S}}\right)$.

Proof. We have that $W^{\mathrm{BM}}\left(\bar{\rho}_{\mathcal{S}}\right)=W(\bar{r}) \circ \prod_{\widetilde{v} \in \mathcal{S}} \iota_{\widetilde{v}}$ by (5.8). The result now follows from Theorem 3.5.2.

5.3.3. Mod $p$ multiplicity one. We continue using the setup from the beginning of Section 5.3. We have the following mod $p$ multiplicity one result.

THEOREM 5.3.4. Let $\tau_{\mathcal{S}}$ and $\sigma \in W^{?}\left(\bar{\rho}_{\mathcal{S}}, \tau_{\mathcal{S}}\right)$ be as in the statement of Theorem 5.1.1. Then

$$
S\left(U^{p} \mathcal{G}\left(\mathcal{O}_{F^{+}, p}\right),\left(\bar{\sigma}\left(\tau_{\mathcal{S}}\right)^{\sigma} \circ \prod_{v \in \Sigma_{p}^{+}} \iota_{\widetilde{v}}\right)^{\vee} \otimes_{\mathcal{O}_{E}} W_{\Sigma_{0}^{+}}\right)[\mathfrak{m}]
$$


is one-dimensional over $\mathbb{F}$.

Proof. By (5.8),

$$
\begin{aligned}
& M_{\infty}\left(\bar{\sigma}\left(\tau_{\mathcal{S}}\right)^{\sigma}\right) / \mathfrak{m} \cong \tilde{M}_{\infty}\left(\bar{\sigma}\left(\tau_{\mathcal{S}}\right)^{\sigma} \circ \prod_{v \in \Sigma_{p}^{+}} \imath \widetilde{v}\right) / \mathfrak{m} \\
& \cong\left(S\left(U^{p} \mathcal{G}\left(\mathcal{O}_{F^{+}, p}\right),\left(\bar{\sigma}\left(\tau_{\mathcal{S}}\right)^{\sigma} \circ \prod_{v \in \Sigma_{p}^{+}} \iota^{\vee}\right)^{\vee} \otimes_{\mathcal{O}} W_{\Sigma_{0}^{+}}\right)[\mathfrak{m}]\right)^{\vee} .
\end{aligned}
$$

By Theorem 5.1.1, the dimension of $M_{\infty}\left(\bar{\sigma}\left(\tau_{\mathcal{S}}\right)^{\sigma}\right) / \mathfrak{m}$ is one.

5.3.4. Lattices in cohomology. Let $r: G_{F} \rightarrow \mathrm{GL}_{3}(E)$ be an automorphic Galois representation as in Theorem 5.3.1. We say that $r$ is minimally ramified if $\left.r\right|_{G_{F_{\tilde{v}}}}$ is minimally ramified in the sense of [CHT08, Definition 2.4.14] for all $v \in \Sigma_{0}^{+}$. Following the notation of [LLHLM18, Section 7.1], let $\lambda$ be the kernel of the system of Hecke eigenvalues $\alpha: \mathbb{T}^{\text {univ }} \rightarrow \mathcal{O}$ associated with $r$, that is, $\alpha$ satisfies

$$
\operatorname{det}\left(1-r^{\vee}\left(\operatorname{Frob}_{w}\right) X\right)=\sum_{j=0}^{3}(-1)^{j}\left(\mathbf{N}_{F / \mathbb{Q}}(w)\right)^{\left(\begin{array}{l}
j \\
2
\end{array}\right)} \alpha\left(T_{w}^{(j)}\right) X^{j}
$$

for all $w$ as above. We now set $W \stackrel{\text { def }}{=} W_{\Sigma_{0}^{+}}$as in Section 5.3.3. By Theorem 5.3.1, $\widetilde{S}\left(U^{p}, W\right)[\lambda]$ is nonzero. Since $r$ is minimally ramified, $r$ corresponds to a prime ideal of $R_{\infty}$ as in [HLM17, Theorem 5.2.1]. By an abuse of notation, we call this ideal $\lambda$. Note that we have that $M_{\infty} / \lambda \cong \widetilde{S}\left(U^{p}, W\right)^{\mathrm{d}} / \lambda$ by (the proof of) [CEG+16, Corollary 2.11].

THEOREM 5.3.5. Let $r: G_{F} \rightarrow \mathrm{GL}_{3}(E)$ be as in Theorem 5.3.1. Assume furthermore that $r$ is minimally ramified. Let $\left\{\tau_{\widetilde{v}}\right\}_{\widetilde{v} \in \mathcal{S}}$ be a 13-generic tame type. The lattice $\sigma(\tau)^{0} \stackrel{\text { def }}{=} \sigma(\tau) \cap \widetilde{S}\left(U^{p}, W\right)[\lambda] \subset \sigma(\tau) \cap \widetilde{S}\left(U^{p}, W\right)[\lambda] \otimes_{\mathcal{O}} E \cong \sigma(\tau)$ depends only on $\left\{\left.r\right|_{G_{F_{\tilde{v}}}}\right\}_{\tilde{v} \in \mathcal{S}}$.

Proof. Let $\bar{\rho}_{\mathcal{S}}$ be $\left(\left.\bar{r}\right|_{G_{F_{\tilde{v}}}}\right)_{\widetilde{v} \in \mathcal{S}}$. Fix $\sigma \in \mathrm{JH}\left(\overline{\sigma\left(\tau_{\mathcal{S}}\right)}\right)$ as in Theorem 5.1.1, a saturated inclusion $\sigma\left(\tau_{\mathcal{S}}\right)^{\sigma} \subset \sigma\left(\tau_{\mathcal{S}}\right)^{0}$, and saturated inclusions $\sigma\left(\tau_{\mathcal{S}}\right)^{\kappa} \subset \sigma\left(\tau_{\mathcal{S}}\right)^{\sigma}$ for all $\kappa \in \mathrm{JH}\left(\overline{\sigma\left(\tau_{\mathcal{S}}\right)}\right)$. Let $\gamma(\kappa) \in \mathcal{O}$ so that $\gamma(\kappa)^{-1} \sigma\left(\tau_{\mathcal{S}}\right)^{\kappa} \subset \sigma\left(\tau_{\mathcal{S}}\right)^{0}$ is saturated. Then $\sigma\left(\tau_{\mathcal{S}}\right)^{0}=\sum_{\kappa \in \mathrm{JH}\left(\overline{\sigma\left(\tau_{\mathcal{S}}\right)} \gamma\right.} \gamma(\kappa)^{-1} \sigma\left(\tau_{\mathcal{S}}\right)^{\kappa}$ for some $\gamma(\kappa) \in \mathcal{O}$ by [EGS15, Lemma 4.1.2]. It suffices to show that for each $\kappa \in \mathrm{JH}\left(\overline{\sigma\left(\tau_{\mathcal{S}}\right)}\right)$, the ideal $(\gamma(\kappa)) \subset \mathcal{O}$ depends only on $\bar{\rho}_{\mathcal{S}}$. 
Observe that the image of the inclusion

$$
\mathcal{O} \cong \operatorname{Hom}_{U_{p}}\left(\sigma\left(\tau_{\mathcal{S}}\right)^{\sigma}, \widetilde{S}\left(U^{p}, W\right)[\lambda]\right) \rightarrow \operatorname{Hom}_{U_{p}}\left(\sigma\left(\tau_{\mathcal{S}}\right)^{\kappa}, \widetilde{S}\left(U^{p}, W\right)[\lambda]\right) \cong \mathcal{O},
$$

is given by the ideal $(\gamma(\kappa))$. By Schikhov duality, the natural inclusion

$$
\begin{aligned}
\mathcal{O} & \cong \operatorname{Hom}_{U_{p}}\left(\left(\widetilde{S}\left(U^{p}, W\right)[\lambda]\right)^{\mathrm{d}},\left(\sigma\left(\tau_{\mathcal{S}}\right)^{\sigma}\right)^{\mathrm{d}}\right) \\
& \rightarrow \operatorname{Hom}_{U_{p}}\left(\left(\widetilde{S}\left(U^{p}, W\right)[\lambda]\right)^{\mathrm{d}},\left(\sigma\left(\tau_{\mathcal{S}}\right)^{\kappa}\right)^{\mathrm{d}}\right) \cong \mathcal{O}
\end{aligned}
$$

is also given by the ideal $(\gamma(\kappa))$. By another application of Schikhov duality, the natural inclusion

$$
\begin{aligned}
\mathcal{O} & \cong \operatorname{Hom}_{U_{p}}\left(\left(\widetilde{S}\left(U^{p}, W\right)[\lambda]\right)^{\mathrm{d}},\left(\sigma\left(\tau_{\mathcal{S}}\right)^{\kappa}\right)^{\mathrm{d}}\right)^{\mathrm{d}} \\
& \rightarrow \operatorname{Hom}_{U_{p}}\left(\left(\widetilde{S}\left(U^{p}, W\right)[\lambda]\right)^{\mathrm{d}},\left(\sigma\left(\tau_{\mathcal{S}}\right)^{\sigma}\right)^{\mathrm{d}}\right)^{\mathrm{d}} \cong \mathcal{O}
\end{aligned}
$$

is also given by the ideal $(\gamma(\kappa))$. Since $\widetilde{S}\left(U^{p}, W\right)[\lambda]^{\mathrm{d}}$ is canonically isomorphic to the torsion-free part of $\widetilde{S}\left(U^{p}, W\right)^{\mathrm{d}} / \lambda$, the ideal $(\gamma(\kappa))$ gives the inclusion

$$
\operatorname{Hom}_{U_{p}}\left(M_{\infty} / \lambda,\left(\sigma\left(\tau_{\mathcal{S}}\right)^{\kappa}\right)^{\mathrm{d}}\right)^{\mathrm{d}} \rightarrow \operatorname{Hom}_{U_{p}}\left(M_{\infty} / \lambda,\left(\sigma\left(\tau_{\mathcal{S}}\right)^{\sigma}\right)^{\mathrm{d}}\right)^{\mathrm{d}} .
$$

Again, note that $\operatorname{Hom}_{U_{p}}\left(M_{\infty} / \lambda,\left(\sigma\left(\tau_{\mathcal{S}}\right)^{\sigma}\right)^{\mathrm{d}}\right)^{\mathrm{d}}$ is isomorphic to (the $p$-torsion-free part of)

$$
\operatorname{Hom}_{U_{p}}\left(M_{\infty},\left(\sigma\left(\tau_{\mathcal{S}}\right)^{\sigma}\right)^{\mathrm{d}}\right)^{\mathrm{d}} / \lambda=M_{\infty}\left(\sigma\left(\tau_{\mathcal{S}}\right)^{\sigma}\right) / \lambda,
$$

and similarly for $\kappa$. So the image of

$$
\mathcal{O} \cong M_{\infty}\left(\sigma\left(\tau_{\mathcal{S}}\right)^{\kappa}\right) / \lambda \rightarrow M_{\infty}\left(\sigma\left(\tau_{\mathcal{S}}\right)^{\sigma}\right) / \lambda \cong \mathcal{O}
$$

is given by the ideal $(\gamma(\kappa))$.

On the other hand, the image of (5.9) is given by

$$
\left(j_{*} j^{*}\left(\prod_{\theta \in W^{?}\left(\bar{\rho}_{\mathcal{S}}\right)} \mathfrak{p}(\theta)^{m(\theta)} R_{\infty}\left(\tau_{\mathcal{S}}\right)\right)\right)\left(M_{\infty}\left(\sigma\left(\tau_{\mathcal{S}}\right)^{\sigma}\right) / \lambda\right)
$$

by Theorem 5.2.3. Then $(\gamma(\kappa))$ is generated by elements in the ideal

$$
j_{*} j^{*}\left(\prod_{\theta \in W^{?}\left(\bar{\rho}_{\mathcal{S}}\right)} \mathfrak{p}(\theta)^{m(\theta)} R_{\infty}\left(\tau_{\mathcal{S}}\right)\right)
$$

modulo the ideal $\lambda$. By Remark 5.2.4, the above ideal comes from $\widehat{\bigotimes}_{\widetilde{v} \in \mathcal{S}} R_{\widetilde{v}}$; hence, the ideal generated by its generators modulo $\lambda$ depends only on $\left\{\left.r\right|_{G_{F_{v}}}\right\}_{\widetilde{v} \in \mathcal{S}}$. 
REMARK 5.3.6. In the hypotheses of Theorem 5.3.5, assume further that $\widetilde{w}\left(\bar{\rho}_{\widetilde{v}}\right.$, $\left.\tau_{\widetilde{v}}\right)_{i}$ has length at least 2 for all $\widetilde{v} \in \mathcal{S}$ and $i \in \mathbb{Z} / f_{\widetilde{v}}$. Then the lattice $\sigma(\tau)^{0}$ can be described explicitly as in [Le18, Theorem 14].

In the following figures, we give a pictorial realization of the gluing procedure appearing in the proofs of Lemmas 5.1.4-5.1.7. Recall that $\widetilde{w}_{i^{\prime}}^{*}=\widetilde{w}\left(\bar{\rho}_{\mathcal{S}}, \tau_{\mathcal{S}}\right)_{i^{\prime}}^{*}$.
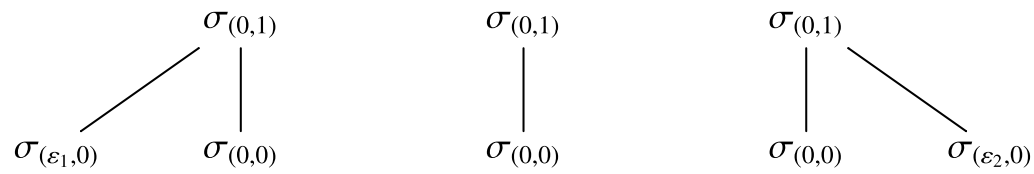

Figure 3. Comparison of $\Sigma_{V^{j}, i^{\prime}}$ in Lemma 5.1.4.

From left to right with arrows pointing down, we have $\Sigma_{V^{1}, i^{\prime}}, \Sigma_{V^{3}, i^{\prime}}$, and $\Sigma_{V^{2}, i^{\prime}}$. The edges correspond to adjacent pairs.
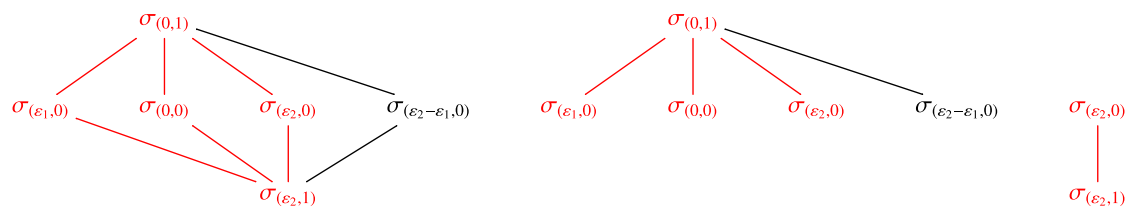

Figure 4. Comparison of $\Sigma_{V, i^{\prime}}$ and $\Sigma_{V^{j}, i^{\prime}}$ in Lemma 5.1.5 when $\widetilde{w}_{i^{\prime}}^{*} t_{-1}=\mathrm{id}$.

Assume $\left(v_{1}, v_{2}, v_{3}\right)=\left(\varepsilon_{1}-\varepsilon_{2}, \varepsilon_{1}, \varepsilon_{1}+\varepsilon_{2}\right)$ and $\widetilde{w}_{i^{\prime}}^{*} t_{-1}=\mathrm{id}$. Then $v_{4}=\varepsilon_{2}$. From left to right with arrows pointing down, we have $\Sigma_{V, i^{\prime}}, \Sigma_{V^{1}, i^{\prime}}$, and $\Sigma_{V^{2}, i^{\prime}}$ (a Weyl segment). In red are the elements in $r\left(\Sigma_{0}\right)$.
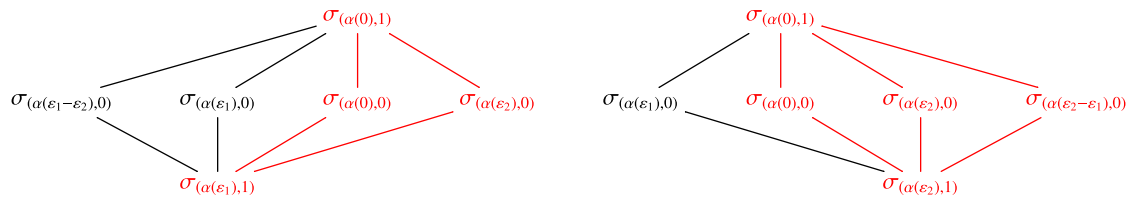

Figure 5. Comparison of $\Sigma_{V^{j}, i^{\prime}}$ in Lemma 5.1.6 when $\widetilde{w}_{i^{\prime}}^{*} t_{-1}=\alpha$.

In the notation of Lemma 5.1.6 consider the case where $v=\varepsilon_{1}+\varepsilon_{2}$ and $\widetilde{w}_{i^{\prime}}^{*} t_{-1}=$ $\alpha$. On the left, we have $\Sigma_{V^{2}, i^{\prime}}$, where we write the elements in $r\left(\Sigma_{0}\right)$ in red. Similarly on the right for $\Sigma_{V^{1}, i^{\prime}}$. 

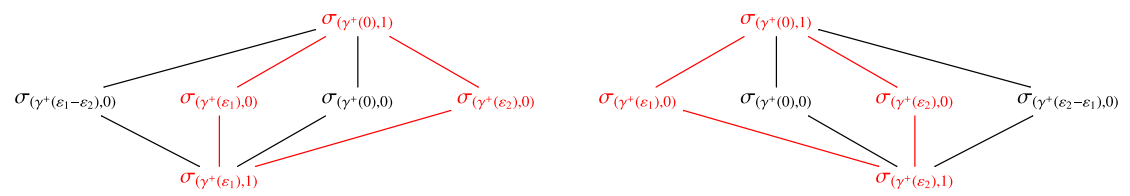

Figure 6. Comparison of $\Sigma_{V^{j}, i^{\prime}}$ in Lemma 5.1.6 when $\widetilde{w}_{i^{\prime}}^{*} t_{-\underline{1}}=\gamma^{+}$.

In the notation of Lemma 5.1.6, consider the case where $v=\varepsilon_{1}+\varepsilon_{2}$ and $\widetilde{w}_{i^{\prime}}^{*} t_{-1}=$ $\gamma^{+}$. On the left, we have $\Sigma_{V^{2}, i^{\prime}}$, where we write the elements in $r\left(\Sigma_{0}\right)$ in red. Similarly on the right for $\Sigma_{V^{1}, i^{\prime}}$.
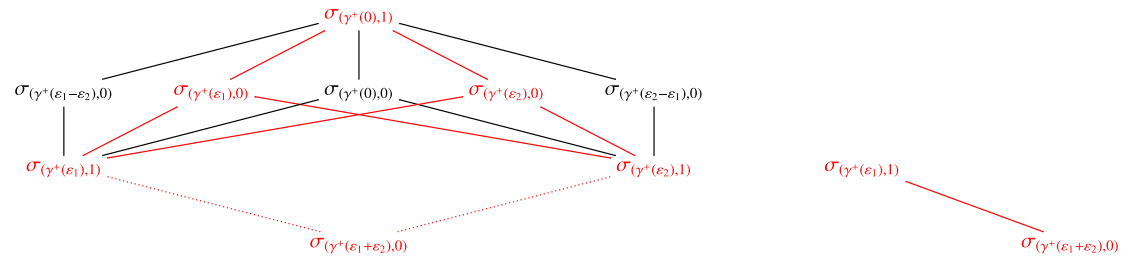

Figure 7. Comparison of $\Sigma_{V^{j}, i^{\prime}}$ in Lemma 5.1.7 when $\widetilde{w}_{i^{\prime}}^{*} t_{-1}=\gamma^{+}$.

In the notation of Lemma 5.1.7, consider the case where $v=\varepsilon_{1}+\varepsilon_{2}$ and $\widetilde{w}_{i^{\prime}}^{*} t_{-1}=$ $\gamma^{+}$. On the left, we have $\Sigma_{V, i^{\prime}}$ (respectively $\Sigma_{V^{1}, i^{\prime}}$ removing the dotted lower part), where we write the elements in $r\left(\Sigma_{0}\right)$ in red. On the right, we have one of the possible choices for $\Sigma_{V^{2}, i^{\prime}}$.

\section{Addendum to [LLHLM18]}

(1) In Theorem 1.1, the statement that 'its special fiber is as predicted by the geometric Breuil-Mézard conjecture' means the following: under the assumptions of Theorem 1.1, the special fiber of $R_{\bar{\rho}}^{(2,1,0), \tau}$ is reduced and the number of irreducible components is less or equal to $\#\left(W^{?}\left(\bar{\rho}^{\mathrm{ss}}\right) \cap\right.$ $\mathrm{JH}(\bar{\sigma}(\tau)))$, with equality if $\bar{\rho}$ is semisimple. This can be checked directly using Theorem 6.14, Tables 3, 7, 8, Propositionsg 8.5, 8.6, and 8.12, and the results of Section 8.2.2.

(2) In Proposition 3.4, the codomain of $T_{\tan }$ should be replaced by

$$
\left\{\left(\rho, \gamma_{0}\right) \mid \rho \in \operatorname{Rep}_{\mathbb{F}^{\prime}[\varepsilon] /\left(\varepsilon^{2}\right)}\left(G_{K_{\infty}}\right), \gamma_{0}: \rho \bmod \varepsilon \stackrel{\sim}{\rightarrow} T_{\mathrm{dd}}^{*}(\overline{\mathfrak{M}})\right\} .
$$

(3) After Equation (3.7), we remark that if $\left(\mathfrak{M}_{A}, \rho_{A}, \delta_{A}\right) \in D_{\overline{\mathfrak{M}}, \bar{\rho}}^{\tau, \square}$, then we have a canonical isomorphism $\mathfrak{M}_{A} \otimes_{A} \mathbb{F} \stackrel{\sim}{\rightarrow} \overline{\mathfrak{M}}$. 
(4) After Definition 4.15, the symbol $Y_{\mathfrak{M}}^{[0,2], \tau}(R)$ denotes the category of pairs $\left(\mathfrak{M}_{R}, J_{R}\right)$ where $\mathfrak{M}_{R} \in Y^{[0, h], \tau}(R)$ and $\jmath_{R}: \mathfrak{M}_{R} \otimes_{R} \mathbb{F} \stackrel{\sim}{\rightarrow} \overline{\mathfrak{M}}$ is an isomorphism in $Y^{[0, h], \tau}(\mathbb{F})$. A similar comment applies to $Y_{\overline{\mathfrak{M}}}^{\mu, \tau}(R)$.

(5) Proof of Theorem 4.17: the ring $R$ is $p$-flat and reduced by [Cal18, Lemma 2.6].

(6) The formula in Lemma 5.2 still converges in $\frac{1}{\lambda} \operatorname{Mat}(R[1 / p] \llbracket u \rrbracket)$ for $R$ a complete local Noetherian flat $\mathcal{O}$-algebra $\mathrm{R}$. While it is possible to show that it lies in $\mathcal{O}_{R}^{\text {rig }}$, for the computations and the arguments in this paper, we only need that its formation is compatible with base change.

(7) In Corollary $5.13, T_{1}, \ldots, T_{8}$ should be replaced by $T_{1}, \ldots, T_{9}$. Similar comment applies to the displayed equation before Theorem 6.14 .

(8) In Section 5.3.2, ' $c_{11} \equiv 0$ modulo $\varpi$ ' should read ' $c_{11}$ and $c_{13} \equiv 0$ modulo the maximal ideal'.

(9) In Section 5.3.3, line -6 and -4 , the $c_{13}^{*}$ in the displayed equations must be replaced by $c_{13}$.

(10) Definition 7.1 should also define automorphic of weight $V$, level $U$, and coefficients $W$ as follows.

DEFINITION 6.0.1. Let $\bar{r}: G_{F} \rightarrow \mathrm{GL}_{3}(\mathbb{F})$ be a continuous Galois representation. Let $V$ be a Serre weight for $\mathcal{G}, U$ be a compact open subgroup of $G\left(\mathbb{A}_{F}^{\infty, p}\right) \times \mathcal{G}\left(\mathcal{O}_{F^{+}, p}\right)$ which is unramified at places $v \mid p$, and $W$ be an $\mathcal{O}$-module with a $U$-action for which the factor $\mathcal{G}\left(\mathcal{O}_{F^{+}, p}\right)$ acts trivially. We say that $\bar{r}$ is automorphic of weight $V$, level $U$, and coefficients $W$ if there exists a cofinite subset $\mathcal{P} \subset \mathcal{P}_{U}$ such that

$$
S(U, V \otimes W)_{\overline{\mathfrak{m}}} \neq 0,
$$

where $\overline{\mathfrak{m}}$ is the kernel of the system of Hecke eigenvalues $\bar{\alpha}: \mathbb{T}_{\mathcal{P}} \rightarrow \mathbb{F}$ associated with $\bar{r}$, and $\bar{\alpha}$ satisfies the equality

$$
\operatorname{det}\left(1-\bar{r}^{\vee}\left(\operatorname{Frob}_{w}\right) X\right)=\sum_{j=0}^{3}(-1)^{j}\left(\mathbf{N}_{F / \mathbb{Q}}(w)\right)^{\left(\begin{array}{c}
j \\
2
\end{array}\right)} \bar{\alpha}\left(T_{w}^{(j)}\right) X^{j}
$$

for all $w \in \mathcal{P}$. We say that $\bar{r}$ is automorphic of weight $V$ (or that $V$ is a Serre weight of $\bar{r}$ ) if $\bar{r}$ is automorphic of weight $V$, level $U$, and coefficients $W$ for some subgroup $U$ and coefficients $W$ as above. We write $W(\bar{r})$ for the set of all Serre weights of $\bar{r}$. We say that $\bar{r}$ is automorphic if $W(\bar{r}) \neq \emptyset$. 
(11) In Definition 7.11(2), 'automorphic of weight $V$ ' should be replaced with 'automorphic of weight $V$, level $U$, and coefficients $W$ ' where $U$ is a fixed compact open subgroup of $G\left(\mathbb{A}_{F}^{\infty, p}\right) \times \mathcal{G}\left(\mathcal{O}_{F^{+}, p}\right)$ which is unramified at places $v \mid p$ and $W$ is a fixed $\mathcal{O}$-module with a $U$-action on which the factor $\mathcal{G}\left(\mathcal{O}_{F^{+}, p}\right)$ acts trivially. This definition of patching functor depends on the implicit choices of $U$ and $W$.

(12) The definition of $e(M)$, given before Proposition 7.14, is incorrect. The correct definition of $e(M)$ is the following: given a finitely generated $R_{\infty}$ module $M$ with scheme-theoretic support $\operatorname{Spec} A$ of dimension at most $d$, define $e(M)$ to be $d$ ! times the coefficient of the degree $d$-term of the Hilbert polynomial of $M$ (considered as an $A$-module).

(13) In the paragraph following the proof of Proposition 7.14, the definition of $\Sigma_{0}$ should exclude primes dividing $p$.

(14) In the proof of Proposition 7.15, 'automorphic of weight $V$ ' should be replaced with 'automorphic of weight $V$, level $U$, and coefficients $W$ ', where $U=\prod_{v \nmid \infty} U_{v}$ and $U_{v}$ is

- $\mathcal{G}\left(\mathcal{O}_{v}\right)$ for $v$ which split in $F$ except for $v_{1}$,

- the preimage of the upper triangular matrices under the map

$$
\mathcal{G}\left(\mathcal{O}_{v}\right) \rightarrow \mathcal{G}\left(k_{v}\right) \underset{{ }_{v}}{\stackrel{\sim}{\rightarrow}} \mathrm{GL}_{3}\left(k_{\widetilde{v}}\right)
$$

if $v=v_{1}$, or

- a maximal hyperspecial maximal compact open subgroup of $G\left(F_{v}\right)$ if $v$ is inert in $F$,

and $W$ is an $\mathcal{O}$-lattice in $\bigotimes_{v \in \Sigma_{0}^{+}} \sigma\left(\tau_{\widetilde{v}}\right) \circ \iota_{\widetilde{v}}$.

(15) In the proof of Proposition 7.16, the tame inertial type $\tau^{\prime}$ should read: $\tau^{\prime} \stackrel{\text { def }}{=}$ $\omega_{2}^{-((b+1)+p(b+1))} \oplus \omega_{2}^{-((c-1)+p a)} \oplus \omega_{2}^{-(a+p(c-1))}$.

(16) The proof of Theorem 7.8 also requires the following proposition, which is a level-lowering result based on techniques in [Tay08]. The proof of Theorem 7.4, which was omitted, uses the same techniques.

Proposition 6.0.2. Let $\bar{r}: G_{F} \rightarrow \mathrm{GL}_{3}(\mathbb{F})$ be a continuous Galois representation with split ramification outside $p$, which is automorphic and satisfies the Taylor-Wiles conditions. If $\bar{r}$ is automorphic of a reachable weight, then it is automorphic of a reachable weight and level $U$ and coefficients $W$, where $U=\prod_{v \nmid \infty} U_{v}$ and $U_{v}$ is 
- $\mathcal{G}\left(\mathcal{O}_{v}\right)$ for $v$ which split in $F$ except for $v_{1}$,

- the preimage of the upper triangular matrices under the map

$$
\mathcal{G}\left(\mathcal{O}_{v}\right) \rightarrow \mathcal{G}\left(k_{v}\right) \underset{v_{v}}{\stackrel{\sim}{\rightarrow}} \mathrm{GL}_{3}\left(k_{\widetilde{v}}\right)
$$

if $v=v_{1}$, or

- a maximal hyperspecial maximal compact open subgroup of $G\left(F_{v}\right)$ if $v$ is inert in $F$,

and $W$ is an $\mathcal{O}$-lattice in $\bigotimes_{v \in \Sigma_{0}^{+}} \sigma\left(\tau_{\widetilde{v}}\right) \circ \iota_{\widetilde{v}}$.

Proof. If $\bar{r}$ is automorphic of a reachable weight $V$, then $S(U, V \otimes W)_{\mathfrak{m}}$ is nonzero for some level $U$ and coefficients $W$. Let $V$ be $\bigotimes_{v} V_{v}$ where $V_{v}=V_{\widetilde{v}} \circ l_{\widetilde{v}}$. Choose tame types $\tau_{v}$ such that

- $\bar{\rho}_{v}$ is admissible with respect to $\tau_{v}$;

- $\ell\left(\boldsymbol{w}\left(\bar{\rho}_{v}, \tau_{v}\right)\right) \geqslant 3$; and

- $V_{\widetilde{v}} \in \mathrm{JH}\left(\bar{\sigma}\left(\tau_{v}\right)\right)$.

Letting $\sigma$ be an $\mathcal{O}$-lattice in $\bigotimes_{v} \sigma\left(\tau_{v}\right) \circ \iota_{\widetilde{v}}$, we have that $S(U, \sigma \otimes W)_{\mathfrak{m}}$ is nonzero. By [EGH13, Lemma 7.1.6 and Theorem 7.2.1], $r \otimes_{\mathbb{F}} \overline{\mathbb{F}}_{p}$ is isomorphic to the reduction of $r_{\pi}: G_{F} \rightarrow \mathrm{GL}_{n}\left(\overline{\mathbb{Q}}_{p}\right)$ for some $\pi$ as in [EGH13, Theorem 7.2.1]. Let $\pi_{F}$ be the regular, algebraic, (conjugate) self dual, cuspidal automorphic representation of $\mathrm{GL}_{n}\left(\mathbb{A}_{F}\right)$ obtained from $\pi$ through base change. Choose a totally real extension $L^{+}$of $F^{+}$such that

- $4 \mid\left[L^{+}: \mathbb{Q}\right]$;

- $L^{+} / F^{+}$is Galois and solvable;

- $L \stackrel{\text { def }}{=} L^{+} F$ is linearly disjoint from $\bar{F}^{\mathrm{ker} \bar{r}}\left(\zeta_{p}\right)$ over $F$;

- $L / L^{+}$is everywhere unramified;

- $p$ is unramified in $L^{+}$;

- $v_{1}$ splits completely in $L$;

- if $\pi_{L}$ is the base change of $\pi_{F}$ to $L$ and $w$ is a place of $L$ lying above $\tilde{v}$ for $v \in \Sigma_{0}^{+}$, then $\pi_{L, w}$ has Iwahori fixed vectors and $\left.\tau_{v}\right|_{I_{L w}}$ is trivial.

One can define analogues of $R_{\tau}^{\text {univ }}$ and $R_{\mathcal{S}}^{\text {univ }}$ from [Gee11, Section 5] as follows. Let $S$ be the union of $\left\{v_{1}\right\}$ and $\Sigma^{+}$. Let $\widetilde{S}$ be the union of $\left\{\widetilde{v}_{1}\right\}$ and $\left\{\widetilde{v}: v \in \Sigma^{+}\right\}$. Let $S_{L}$ be the set of places in $L^{+}$lying above places in $S$, and let $\widetilde{S}_{L}$ be the set of places in $L$ lying above places in $\widetilde{S}$. Let $\widetilde{\Sigma}_{L, p}$ be the set 
of places in $L$ lying over a place in $\widetilde{\Sigma}_{p}^{+}, \widetilde{\Sigma}_{L, 0}$ the set of places in $L$ lying over a place in $\widetilde{\Sigma}_{0}^{+}$, and $\widetilde{\Sigma}_{L, 1}$ be the set of places in $L$ lying over $\widetilde{v}_{1}$. Let $\widetilde{\Sigma}_{L}$ be the union of $\widetilde{\Sigma}_{L, p}$ and $\widetilde{\Sigma}_{L, 0}$. If $\widetilde{v} \in \widetilde{\Sigma}_{L, p}$, let $\tau_{\widetilde{v}}$ be $\left.\tau_{\left.\widetilde{v}\right|_{F}}\right|_{I_{L}}$. If $\widetilde{v} \in \widetilde{\Sigma}_{L, 0}$, let $R^{\square, \tau_{\tilde{v}}}$ be the lifting ring for $\left.\bar{r}\right|_{G_{L_{\tilde{v}}}}$ parametrizing lifts whose characteristic polynomial is $(X-1)^{3}$. We let $R_{\tau}^{\text {univ }}$ be the universal deformation ring corresponding to the deformation problem

$$
\left(F / F^{+}, S, \widetilde{S}, \mathcal{O}_{E}, \bar{r}, \epsilon^{-2} \delta_{F / F^{+}},\left\{R_{\widetilde{v}_{1}}^{\square}\right\} \cup\left\{R_{\widetilde{v}}^{\square, \tau_{\widetilde{v}}}\right\}_{v \in \Sigma^{+}}\right),
$$

and we let $R_{\mathcal{S}}^{\text {univ }}$ be the universal deformation ring corresponding to the deformation problem

$$
\left(L / L^{+}, S_{L}, \widetilde{S}_{L}, \mathcal{O}_{E},\left.\bar{r}\right|_{G_{L}}, \epsilon^{-2} \delta_{L / L^{+}},\left\{R_{\widetilde{u}_{1}}^{\square}\right\}_{\widetilde{u}_{1} \in \widetilde{S}_{L, 1}} \cup\left\{R_{\widetilde{v}}^{\square, \tau_{v}}\right\}_{\widetilde{v} \in \widetilde{\Sigma}_{L}}\right) .
$$

The proof of [Gee11, Theorem 5.1.4] shows that $R_{\tau}^{\text {univ }}$ is finite over $\mathcal{O}_{E}$. Indeed, $R_{\tau}^{\text {univ }}$ is finite over $R_{\mathcal{S}}^{\text {univ }}$ and $R_{\mathcal{S}}^{\text {univ }}$ is finite over $\mathcal{O}_{E}$ since $\left(R_{\mathcal{S}}^{\text {univ }}\right)^{\text {red }}$ is isomorphic to an appropriate Hecke algebra by the proof of [Gue11, Theorem 3.4]. One replaces Fontaine-Laffaille deformation rings with $R^{\square, \tau \widetilde{v}}$ for $\widetilde{v} \in \widetilde{\Sigma}_{L, p}$, which is geometrically integral by [LLHLM18, Section 5.3].

That $R_{\tau}^{\text {univ }}$ is finite over $\mathcal{O}_{E}$ implies that there is a conjugate self-dual lift $r: G_{F} \rightarrow \mathrm{GL}_{3}\left(\overline{\mathbb{Q}}_{p}\right)$ of $\bar{r}$ which is minimally ramified outside $p$ and potentially crystalline of type $\left((0,1,2), \tau_{v}\right)$ at $\widetilde{v}$ for each $v \in \Sigma_{0}^{+}$. Moreover, we have that the restriction $\left.r\right|_{G_{L}}$, which corresponds to a point of Spec $R_{\mathcal{S}}^{\text {univ }}$, is automorphic. Solvable base change then implies that $r$ is automorphic. Local-global compatibility implies that $S(U, \sigma \otimes W)_{\mathfrak{m}}$ is nonzero for $U$ and $W$ as in the statement of the proposition. Then $S(U$, $\left.V^{\prime} \otimes W\right)_{\mathfrak{m}}$ is nonzero for some reachable $V^{\prime} \in \mathrm{JH}(\bar{\sigma})$.

This would then show that $M_{\infty}\left(\bigotimes_{v \in \Sigma_{p}^{+}} \bar{\sigma}\left(\tau_{v}\right)^{\circ}\right)$ is nonzero in the third paragraph (one cannot directly cite Definition 7.11(2) because of the change above).

(17) In Section 8.1, after the proof of Lemma 8.2, the $\mathcal{O}$-algebra $R_{\overline{\mathfrak{M}}, \bar{\rho}}^{\tau, \bar{\beta}, \square}$ has relative dimension 15 over $\mathcal{O}$.

(18) In Corollary 8.4, there should be the further relation $c_{12} c_{33}=0$.

(19) The final sentence of Corollary 8.4 should be replaced by the claim that the ring $R_{\overline{\mathfrak{M}}, \bar{\rho}}^{\tau, \bar{\beta}} / \varpi$ is a quotient of $\widetilde{R} \llbracket c_{i i}^{*}-\left[\bar{c}_{i i}^{*}\right], x_{j}, 1 \leqslant i \leqslant 3,1 \leqslant j \leqslant$ 
91]. This comment applies also to Proposition 8.11. These changes justify the dimension hypothesis in Lemma 8.8, which is used in the proof of Proposition 8.6.

(20) In the proof of Proposition 8.11, we remark that $c_{33}=c_{23} c_{31}\left(c_{21}^{*}\right)^{-1}$.

(21) In the caption of Table 4, the coefficients are in $\mathbb{F}$.

(22) In the caption of Table 5, the coefficients are in $R$.

(23) The entry $(1,3)$ of Table 6 should read 'Leading term of the monodromy condition'.

(24) The missing entries in the second column of Table 6 can be read off from Table 5 and the missing entry in the third column of Table 6 can be read off from Proposition 8.3.

(25) In Table 6, the leading term of the monodromy condition for $\alpha \beta \alpha \gamma$, the second $c_{33}^{*}$ should be removed. Moreover, in the caption, the $a_{s_{j+1}(i)}^{(j)}$ for $i=1,2,3$ should be bold.

\section{Acknowledgements}

We would like to thank Christophe Breuil, James Humphreys, and Cornelius Pillen for many helpful conversations. We would also like to thank Matthew Emerton, Toby Gee, and Florian Herzig for their support, guidance, and for comments on an earlier draft of this paper. Part of this work was carried out while the authors were visiting the Institut Henri Poincare and the Mathematisches Forschungsinstitut Oberwolfach, and we would like to thank these institutions for their hospitality. BLH acknowledges support from the National Science Foundation under grant Nos. DMS-1128155, DMS-1802037 and the Alfred P. Sloan Foundation. DL was supported by the National Science Foundation under agreement Nos. DMS-1128155 and DMS-1703182 and an AMS-Simons travel grant. Finally, the authors express their utmost gratitude to the anonymous referee for his or her meticulous and patient reading of several versions of this paper. The reports were invaluable in helping us to improve the quality, precision, and clarity of this paper.

Conflict of Interest: The authors declare no conflict of interest. 


\section{References}

[Alp86] J. L. Alperin, 'Modular representations as an introduction to the local representation theory of finite groups', in Local Representation Theory, Cambridge Studies in Advanced Mathematics, 11 (Cambridge University Press, Cambridge, 1986).

[AM01] H. H. Andersen and K. Masaharu, 'Filtrations on $G_{1} T$-modules', Proc. Lond. Math. Soc. (3) 82(3) (2001), 614-646.

[And87] H. H. Andersen, 'Extensions of simple modules for finite Chevalley groups', J. Algebra 111(2) (1987), 388-403.

[BDM15] C. Bowman, S. R. Doty and S. Martin, 'Decomposition of tensor products of modular irreducible representations for $\mathrm{SL}_{3}$ : the $p \geqslant 5$ case', Int. Electron. J. Algebra 17 (2015), 105-138.

[BP13] C. Breuil and V. Paskunas, 'Towards a modulo $p$ Langlands correspondence for GL $_{2}$ ', Mem. Amer. Math. Soc. 216 (2012), no. 1016, vi+114 pp.

[Bre14] C. Breuil, 'Sur un problème de compatibilité local-global modulo $p$ pour $\mathrm{GL}_{2}$ ', J. Reine Angew. Math. 692 (2014), 1-76.

[Cal18] F. Calegari, 'Non-minimal modularity lifting in weight one', J. Reine Angew. Math. 740 (2018), 41-62.

[CDT99] B. Conrad, F. Diamond and R. Taylor, 'Modularity of certain potentially BarsottiTate Galois representations', J. Amer. Math. Soc. 12(2) (1999), 521-567.

[CEG+16] A. Caraiani, M. Emerton, T. Gee, D. Geraghty, V. Paskūnas and S. W. Shin, 'Patching and the $p$-adic local Langlands correspondence', Camb. J. Math. 4(2) (2016), 197-287.

[CHT08] L. Clozel, M. Harris and R. Taylor, 'Automorphy for some $l$-adic lifts of automorphic mod $l$ Galois representations', Publ. Math. Inst. Hautes Études Sci. 108 (2008), 1-181. With Appendix A, summarizing unpublished work of Russ Mann, and Appendix B by Marie-France Vignéras.

[CL18] A. Caraiani and B. Levin, 'Kisin modules with descent data and parahoric local models', Ann. Sci. Éc. Norm. Supér. (4) 51(1) (2018), 181-213.

[Dia97] F. Diamond, 'The Taylor-Wiles construction and multiplicity one', Invent. Math. 128(2) (1997), 379-391.

[DL76] P. Deligne and G. Lusztig, 'Representations of reductive groups over finite fields', Ann. of Math. (2) 103(1) (1976), 103-161.

[EG14] M. Emerton and T. Gee, 'A geometric perspective on the Breuil-Mézard conjecture', J. Inst. Math. Jussieu 13(1) (2014), 183-223.

[EGH13] M. Emerton, T. Gee and F. Herzig, 'Weight cycling and Serre-type conjectures for unitary groups', Duke Math. J. 162(9) (2013), 1649-1722.

[EGS15] M. Emerton, T. Gee and D. Savitt, 'Lattices in the cohomology of Shimura curves', Invent. Math. 200(1) (2015), 1-96.

[Enn] J. Enns, 'On weight elimination for $\mathrm{GL}_{n}\left(\mathbf{Q}_{p^{f}}\right)$ ', Math. Res. Lett. 26(1) (2019), 53-66.

[Gee11] T. Gee, 'Automorphic lifts of prescribed types', Math. Ann. 350(1) (2011), 107-144.

[GHS18] T. Gee, F. Herzig and D. Savitt, 'General Serre weight conjectures', J. Eur. Math. Soc. (JEMS) 20(12) (2018), 2859-2949.

[Gro65] A. Grothendieck, 'Éléments de géométrie algébrique. IV. Étude locale des schémas et des morphismes de schémas. II', Publ. Math. Inst. Hautes Études Sci. 24 (1965), 231. 
[Gue11] L. Guerberoff, 'Modularity lifting theorems for Galois representations of unitary type', Compos. Math. 147(4) (2011), 1022-1058.

[Her09] F. Herzig, 'The weight in a Serre-type conjecture for tame $n$-dimensional Galois representations', Duke Math. J. 149(1) (2009), 37-116.

[HK04] B. Hassett and S. J. Kovács, 'Reflexive pull-backs and base extension', J. Algebraic Geom. 13(2) (2004), 233-247.

[HLM17] F. Herzig, D. Le and S. Morra, 'On mod $p$ local-global compatibility for $\mathrm{GL}_{3}$ in the ordinary case', Compos. Math. 153(11) (2017), 2215-2286.

[Hum06] J. E. Humphreys, Modular Representations of Finite Groups of Lie Type, London Mathematical Society Lecture Note Series, 326 (Cambridge University Press, Cambridge, 2006).

[Jan03] J. C. Jantzen, Representations of Algebraic Groups, second edn, Mathematical Surveys and Monographs, 107 (American Mathematical Society, Providence, RI, 2003).

[Kis08] M. Kisin, 'Potentially semi-stable deformation rings', J. Amer. Math. Soc. 21(2) (2008), 513-546.

[Le18] D. Le, 'Lattices in the cohomology of $U(3)$ arithmetic manifolds', Math. Ann. 372(1-2) (2018), 55-89.

[LLHL19] D. Le, B. V. Le Hung and B. Levin, 'Weight elimination in Serre-type conjectures', Duke Math. J. 168(13) (2019), 2433-2506.

[LLHLM18] D. Le, B. V. Le Hung, B. Levin and S. Morra, 'Potentially crystalline deformation rings and Serre weight conjectures: shapes and shadows', Invent. Math. 212(1) (2018), 1-107.

[Pi193] C. Pillen, 'Reduction modulo $p$ of some Deligne-Lusztig characters', Arch. Math. (Basel) 61(5) (1993), 421-433.

[Pi197] 'Loewy series for principal series representations of finite Chevalley groups', J. Algebra 189(1) (1997), 101-124.

[Sta19] The Stacks Project Authors, Stacks Project, https://stacks.math.columbia.edu, 2019.

[Tay08] R. Taylor, 'Automorphy for some $l$-adic lifts of automorphic mod $l$ Galois representations. II', Publ. Math. Inst. Hautes Études Sci. 108 (2008), 183-239. 